\title{
3D SILICON NANOPHOTONICS
}

\author{
DRIEDIMENSIONALE \\ NANOFOTONICA IN SILICIUM
}


Promotiecommissie

Promotor

Overige leden
Prof. Dr. W. L. Vos

Prof. Dr. Ir. J.P.H. Benschop (ASML and University of Twente)

Dr. P. Cloetens (European Synchrotron Radiation Facility)

Prof. Dr. A. Lagendijk (University of Twente and University of Amsterdam) Prof. Dr. Ir. A.J.H.M. Rijnders (University of Twente)

Prof. Dr. F. Scheffold (University of Fribourg)

This work was financially supported by the FOM program "Stirring of light!" and MESA+.

It was carried out at the Complex Photonic Systems (COPS) chair, Department of Science and Technology and MESA+ Institute for Nanotechnology,

University of Twente, P.O. Box 217, 7500 AE Enschede, The Netherlands.

This thesis can be downloaded from http://www.photonicbandgaps.com

ISBN: 978-90-365-4374-3

URL: https://doi.org/10.3990/1.9789036543743 


\section{D SILICON NANOPHOTONICS DRIEDIMENSIONALE NANOFOTONICA IN SILICIUM}

\section{PROEFSCHRIFT}

ter verkrijging van

de graad van doctor aan de Universiteit Twente, op gezag van de rector magnificus,

Prof. dr. T.T.M. Palstra,

volgens besluit van het College voor Promoties

in het openbaar te verdedigen

op donderdag 13 Juli 2017 om 14.45 uur

door

\section{Diana Grishina}

geboren op 1 November 1990

te Kurovskoye, USSR 
Dit proefschrift is goedgekeurd door:

Prof. Dr. W. L. Vos 
In memoriam of my father Alexander

and

my aunt Kate 



\section{Contents}

1 Introduction 9

1.1 Photonic crystals . . . . . . . . . . . . . . . . . 10

1.2 Photonic strength . . . . . . . . . . . . . . . . . 11

1.3 Realization and probing of photonic crystals . . . . . . . . . . . 14

2 Single-step etch mask for 3D monolithic nanostructures 27

2.1 Introduction . . . . . . . . . . . . . . . . . . . . 27

2.2 Fabrication process for the 3D single-step etch mask with built-in alignment . . . . . . . . . . . . . . . . . . . . . . . . . . . . . . 38

2.3 Results and discussion . . . . . . . . . . . . . . . . . . . 31

2.3.1 Fabricated structure characterization . . . . . . . . . . 31

2.3.2 Structures feasible for fabrication . . . . . . . . . . . 35

2.4 Conclusions and outlook . . . . . . . . . . . . . . . 38

3 Deep UV lithography on the inclined sidewall of a wafer 43

3.1 Introduction . . . . . . . . . . . . . . . . . . . 43

3.2 Experimental results . . . . . . . . . . . . . . . . . . . . . . 45

3.2.1 Process flow for V-trench fabrication . . . . . . . . . . 45

3.2.2 Spin coating of the sidewalls with photoresist . . . . . . . 48

3.2.3 DUV exposure with step-and-scan lithography . . . . . . . 49

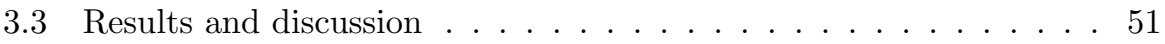

3.4 Summary and outlook . . . . . . . . . . . . . . . 54

4 Deep reactive ion etching of silicon $\quad 59$

4.1 Introduction . . . . . . . . . . . . . . . . . . . . 59

4.2 General description of deep reactive ion etching . . . . . . . . . . 60

4.3 Experimental . . . . . . . . . . . . . . . . 61

4.4 Ultra-high aspect ratio nanopores in $\mathrm{Si}$. . . . . . . . . . . . . . . . 62

4.5 Advanced two-sided etching for 3D nanofabrication . . . . . . . . 65

4.5.1 Choice of ICP power . . . . . . . . . . . . . . . . 66

4.5.2 Polymer residuals after the Bosch process . . . . . . . . . 70

4.5.3 Process flow transfer to SPTS Pegasus etcher . . . . . . . 72

4.6 Summary . . . . . . . . . . . . . . . . . 72

5 Looking inside 3D photonic nanostructures with X-ray tomography 77

5.1 Introduction . . . . . . . . . . . . . . . . . . 77

5.2 Zoom nanotomography . . . . . . . . . . . . . . 78

5.2 .1 General scheme . . . . . . . . . . . . . . 78 
5.2.2 Phase retrieval .................. 80

5.3 Experimental . . . . . . . . . . . . . . . . 86

5.3 .1 Samples ....................... 86

5.3.2 Experimental setup of beamline . . . . . . . . . . . 88

5.4 Results and discussions . . . . . . . . . . . . . . . . 89

5.4.1 Choice of the phase retrieval algorithm . . . . . . . . 89

5.4.2 Choice of the resolution ............... . 93

5.4.3 Results and discussion on photonic crystals . . . . . . . 96

5.5 Summary . . . . . . . . . . . . . . . . . . 102

6 Reflectivity of silicon photonic crystals with and without embedded $\begin{array}{ll}\text { point defects } & 109\end{array}$

6.1 Introduction . . . . . . . . . . . . . . . . . . . . . . 109

6.2 Optical setup for broadband reflectivity . . . . . . . . . . . . . 111

6.3 Reflectivity of $2 \mathrm{D}$ silicon photonic crystals . . . . . . . . . . . . . 112

6.4 Reflectivity study of 3D silicon photonic band gap crystals . . . . . 120

6.4.1 Inverse woodpile photonic crystal . . . . . . . . . . . . 120

6.4.2 Reflectivity of 3D inverse woodpile photonic crystal without embedded defects . . . . . . . . . . . . . . 121

6.4.3 Signature of the cavity resonance in the 3D band gap . . . 125

6.5 Summary . . . . . . . . . . . . . . . . . 129

$\begin{array}{lll}7 & \text { Outlook } & 135\end{array}$

$\begin{array}{lr}\text { A Background on X-ray wave propagation } & 139\end{array}$

A.1 Basic equations. . . . . . . . . . . . . . . . . . . . . . . . . . 139

A.2 Kirchhoff diffraction . . . . . . . . . . . . . . . . . . . 141

A.3 Rayleigh-Sommerfeld diffraction . . . . . . . . . . . . . . . . . 141

A.4 Fresnel diffraction . . . . . . . . . . . . . . . . . . . . . . . . . . . . . . . . . . . . . . . . . . .

A.5 Fraunhofer diffraction . . . . . . . . . . . . . . . . . . 143

A.6 Talbot effect for amplitude grating . . . . . . . . . . . . . . . . . . 143

A.7 Talbot effect for a phase grating . . . . . . . . . . . . . . . . . 145

A.7.1 Numerical calculation in the Fresnel regime. . . . . . . . . . 145

A.7.2 Diffraction using transfer function approach . . . . . . . . . 147

A.8 Diffraction pattern from a 2D centered rectangular grating . . . . . 150

B Sample list

C Wave propagation in the birefringent media $\quad 159$

C.1 Wave propagation in the birefringent media . . . . . . . . . . . . 159

C.1.1 TE polarization . . . . . . . . . . . . . . . . 161

C.1.2 TM polarization . . . . . . . . . . . . . . 161

C.1.3 Reflection and refraction from birefringent medium . . . . . 162

$\begin{array}{lr}\text { Nederlandse samenvatting } & 167\end{array}$

$\begin{array}{ll}\text { Acknowledgements } & 169\end{array}$ 


\section{CHAPTER 1}

\section{Introduction}

Light is everywhere! It is the essence of life on our planet. Light from the sun is the main source of energy for the plants that are at the very bottom of the food chain hence it is the foundation of all life on earth. Daily and nightly changes in the intensity of light are the control factors for many physiological processes in humans and animals bodies. Obviously, light is widely used in society and science $[1,2]$. Even the fact that we observe the outside world with our eyes is based on the existence of light and its reflection and refraction from our surroundings. Being limited by the resolution and sensitivity of eyes, mankind creates special devices to obtain the information that is otherwise hidden.

Light is widely used for sensing [3-5]. Sensors that allow us to detect the presence of small molecules or micro organisms in the air, gases, our bodies or environment, which is important for medical or industrial purposes. Nanophotonic sensors are compact, versatile, fast and sensitive $[6,7]$.

Light has also always attracted great attention due to its fundamental property to have the fastest speed at which information or matter can travel [8]. Thus it is natural to use light for communication that has a great importance for modern society. Already in ancient times light was used for communication in the form of for example lighthouses. The most famous Pharos lighthouse of Alexandria is dated between 280 and $247 \mathrm{BC}$ [9]. But the larger a society grows, the further distances information needs to travel to be communicated. In modern world it is necessary to transmit information as fast as possible over distances of thousands of kilometers. The use of optical circuits for communication started to be a major subject of research in the 19060s and 1970s [10, 11].

Due to the possible integration of optical circuits to the existing electronic devices, silicon stands out as a promising material for optical circuits [12, 13]. In the 1990's silicon optoelectronics started to grow from idea to the realization $[14,15]$. Silicon optical phase modulators with the modulation frequency rising from $\tilde{2} 0 \mathrm{MHz}$ [16] in 1990's to $1 \mathrm{GHz}$ [17] in 2000's. To allow monolithic integration of silicon modulators with electronics on a single silicon substrate it is important to keep the technology compatible with the widely-used complimentary metal-oxide-semiconductor (CMOS) processing. Moreover, considering the small size of modern electronic components, in order to match the length scales with it photonic devices have to be of nanometer sizes. Combination of light and silicon at nanometer scale brings us to silicon nanophotonics that is a subject of current thesis. Currently the main application of silicon nanophotonics is to send the signal from one chip to another by optical means, called optical interconnec- 
tion [18] and this is being extended to on-chip communication called datacom [19]. Using optical elements allows to benefit in interconnect density, energy and timing [20]. Silicon CMOS-integrated nanophotonics allows to benefit from large total bandwidth with a high number of channels controlled in a wavelengthdivision multiplexer (WDM) and cost efficiency[21, 22]. Up to date most integrated optics and CMOS processes remain planar and thus two-dimensional (2D). To continue improving the performance and decreasing the costs, higher levels of integration are needed. Three-dimensional (3D) integration is road leading to the future developments. Examples of commercially available integrated circuits with 3D architecture are being offered by world leading companies such as Intel and Micron[23] with a recently announced 3D X-point technology for memory cells and Samsung with 3D vertical NAND (v-NAND) [24].

Besides an increasing commercial interest to push technologies to three dimensions, many fundamental questions arise when the materials are structured in a $3 \mathrm{D}$ way. An example of phenomena occurring only in $3 \mathrm{D}$ nanostructures is the physics of a full 3D photonic band gap corresponding to a range of frequencies of light are forbidden to propagate in any direction and for any polarization, leading to the ultimate control of spontaneous emission [25], and propagation. Another 3D aspect of light is the so-called "stirring" of light, in other words manipulating the phase space of light [26] that is pursued in the FOM program "Stirring of light!" ${ }^{1}$. Current open questions are whether 3D photonic crystals with embedded defects are suitable media for the long awaited experimental observation of 3D Anderson localization of light [28, 29] or even suitable building blocks for 3D integrated circuits [30]. To fulfill these prospects there needs to be a way to fabricate in a CMOS-compatible manner 3D photonic crystals in a large scale to prevent finite size effects and fulfill industrial demands and with good alignment for controlling the geometries. Therefore in this thesis we propose a "wild" idea to provide a CMOS compatible platform to realize truly 3D silicon nanophotonics.

\subsection{Photonic crystals}

Photonic crystals are ordered composite materials with a periodic change of the refractive index on the scale of the wavelength of light $\lambda[25,29]$. Such a periodicity results in their unique properties in controlling light propagation. At the Bragg condition [31] light that is reflected off the lattice planes in the crystal interferes constructively. The wavelength $\lambda_{\text {Bragg }}$ at which constructive interference of reflected light is equal to

$$
n \cdot \lambda_{\text {Bragg }}=2 \cdot n_{e f f} \cdot d_{h k l} \cdot \sin \theta
$$

where $n$ is the diffraction order, $n_{\text {eff }}$ is the effective refractive index of the medium, $d_{h k l}$ is the lattice parameter, and $\theta$ is the incident angle. A range of frequencies for which light is forbidden to propagate in an infinite crystal due

\footnotetext{
${ }^{1}$ To steer light one needs both advanced wavefront shaping [27] and advanced 3D nanostrcutures; this dissertation focuses on the latter
} 
to Bragg interference is called a stop gap. Constructive interference of the reflected light results in the observed increase of the reflectivity for a range of frequencies that is referred to as a stop band. Stop bands for visible light can be observed by eye as shiny and colorful appearance that change its color depending on the viewing angle, as for example natural opals or butterfly wings. In the special case that materials are periodically arranged in all three dimensions there exist a complete 3D band gap - a range of frequencies in which light is forbidden to propagate in all directions and for all polarizations. The term band gap originates from the analogy with semiconductors exhibiting a band gap where no electron states are allowed [32]. The existence of a complete photonic band gap allows photonic crystals to exhibit unique optical properties and novel phenomena in cavity quantum electrodynamics (cQED)[33-35]. Fundamental property of a complete photonic band gap is a suppression of vacuum fluctuations [36]. Such suppression of vacuum fluctuations results in the complete inhibition of spontaneous emission in the band gap of a photonic crystal [36]. Photonic crystals with a complete photonic band gap will also modify blackbody radiation spectrum [37], affect dipole-dipole interactions including F'orster transfer [38] and the van der Waals and Casimir forces [39, 40] and provide shielding of quantum computing systems from vacuum fluctuations to make them more robust against vacuum noise [35, 41].

In analogy with doping of semiconductors, introducing intended defects and thus states in a photonic band gap enriches cQED even more. A single point defect in a photonic band gap results in an ultimate $3 \mathrm{D}$ cavity that is shielded in all dimensions by a photonic crystal such that there is no direction where light can leak sometimes called a "nano-box" for light [42, 43]. To achieve a high quality factor of a cavity it is needed to surround it with large enough photonic crystal to provide sufficient shielding. The picture ibecomes even more interesting when there is an array of cavities in the photonic crystal. Light then has a possibility to exist in the multiple tiny "nano-boxes" located inside photonic crystal or we can even "stir" it to hop from one cavity to another, as is depicted in Figure 1.1. The array of point defects inside a complete photonic band gap may be a suitable medium for the experimental observation of 3D Anderson localization of light [29].

\subsection{Photonic strength}

An important parameter that determines how strongly light interacts with any photonic structure is the photonic strength [44]. The photonic strength is defined as the ratio of the polarizability $\alpha$ of an average scatterer and the average volume per scatterer $V$ :

$$
S \equiv \frac{4 \pi \alpha}{V}
$$

For the case of photonic crystals, the photonic strength can be written as [45]:

$$
S=3 \phi \frac{m^{2}-1}{m^{2}+2} 3 g_{h k l}
$$




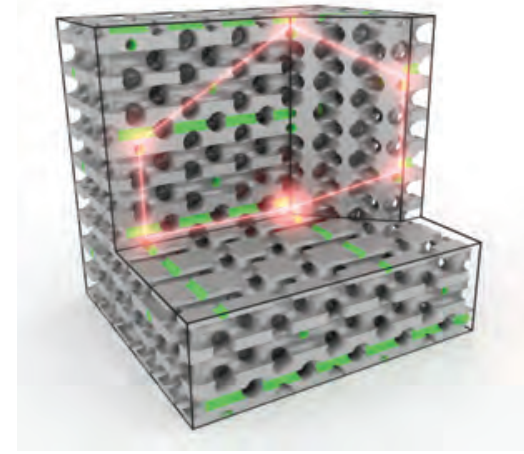

Figure 1.1: Artistic representation of a crystal of cavities inside the 3D photonic crystal. Each cavity is formed as an intersection of smaller pores (green) and a number are lighted in the picture. Light is shown to be "hopping" from one cavity to another as shown with the bright lines which is radically different from free-space propagation of light.

where $\phi$ is the volume fraction of high refractive index material, $g_{h l k}$ is the structure factor at the dominant reciprocal lattice vector that depends on the geometry of the crystal and $m$ is the refractive index contrast of the high $\left(n_{\text {high }}\right)$ and low $\left(n_{\text {low }}\right)$ refractive index materials:

$$
m \equiv \frac{n_{\text {high }}}{n_{\text {low }}} .
$$

From equation 1.3 it is clear that in order to achieve high photonic strength a large refractive index contrast $m$ is needed. This makes a choice of silicon with the high refractive index $n_{s i}=3.5$ even more attractive for photonic crystal fabrication which is the main choice of this thesis. In practice photonic strength $S$ can be determined from the relative width of the dominant stop band observed in transmission or reflectivity experiments $\Delta \omega$ and the central frequency of the stop band $\omega_{c}$ :

$$
S_{\text {exp }} \simeq \frac{\Delta \omega_{s . b a n d}}{\omega_{c}}
$$

The central frequency $\omega_{c}$ can be expressed as $\omega_{c}=\frac{2 \pi c}{\lambda_{\text {Bragg }}}$, where $\lambda_{\text {Bragg }}$ is the central wavelength of the measured stop band. The photonic strength also defines the length scale $L_{B}$ called Bragg length for the photonic crystal, which is the exponential decay length of incident light at the central wavelength of the stop band. The Bragg length also provides a typical length scale at which interference of light occurs in a photonic crystal. These two quantities $S$ and $L_{B}$ are related as

$$
L_{B}=\frac{2 d}{\pi S}
$$

where $d$ is the spacing between the crystal planes. In order to increase photonic strength there are few approaches. One is to increase volume fraction of high 
refractive index material $\phi$ according to equation 1.3. At the same time it is noted that $100 \%$ of high refractive index material will not lead to the high photonic strength as there will be no refractive index contrast. Therefore it is clear that there is am optimal volume fraction.

A second way to improve photonic strength is to choose the crystal geometry that provides wide photonic band gap. Diamond-like structures have attracted particular attention due to their large predicted band gap [46, 47]. The practical realization of diamond-like crystals include woodpiles and inverse woodpiles introduced in Reference [46], spiral diamonds [48] and others. A most promis-

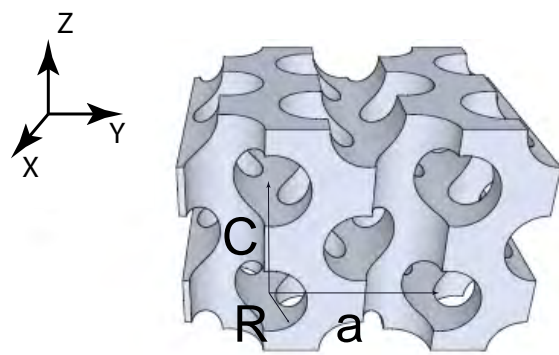

Figure 1.2: The structure of $3 \mathrm{D}$ inverse woodpile photonic crystals consists of two identical arrays of pores with radius $R$ that are running in two perpendicular directions $X$ and $Z$ with lattice parameters $a$ and $c$. The arrays are aligned such that pores running in the $Z$-direction are centered between rows of pores in the $X$-direction.

ing type of photonic crystal structure is the inverse woodpile due to its large photonic strength corresponding to a wide relative band width of more than $\frac{\Delta \omega}{\omega_{c}}=25 \%[49,50]$. In this thesis we have chosen to study inverse woodpile photonic crystals whose structure consists of two perpendicular 2D arrays of pores. Each array is a centered rectangular lattice with parameters $(a, c)$ such that $a / c=\sqrt{2}$ corresponding to a cubic diamond $\{h k l=110\}$ face, as is shown in Figure 1.2. Typical values of the pore radius $R$ and lattice constant $a$ are $R=160$ $\mathrm{nm}$ and $a=680 \mathrm{~nm}$. The pores are aligned such that the pores running in the $X$ direction are centered between the rows of pores running in the $Z$-direction. For the inverse woodpile photonic structure the dependence of the volume fraction of Si $\phi$ on the reduced pore radius $R / a$ is shown in Figure 1.3. By calculation the band diagram for each $R / a$ for inverse woodpile crystal structure we obtain the photonic strength $S$ depending on $R / a$ (Figure 1.4). Figure 1.4 shows the upper and lower edges of the stop gap in the $\Gamma X$ and $\Gamma Z$ high symmetry directions and the resulting photonic strength $\frac{\Delta \omega}{\omega_{c}}$. It is clear from Figure 1.4 that there exist an optimum $R / a$ providing the maximum photonic strength and the widest band gap. The optimum value for the infinite inverse woodpile photonic crystal is found to be $R / a_{\text {opt }}=0.245[49,50]$. In this thesis we aim to vary photonic strength by varying the pore radius $R / a$ at constant lattice parameter $a$. 


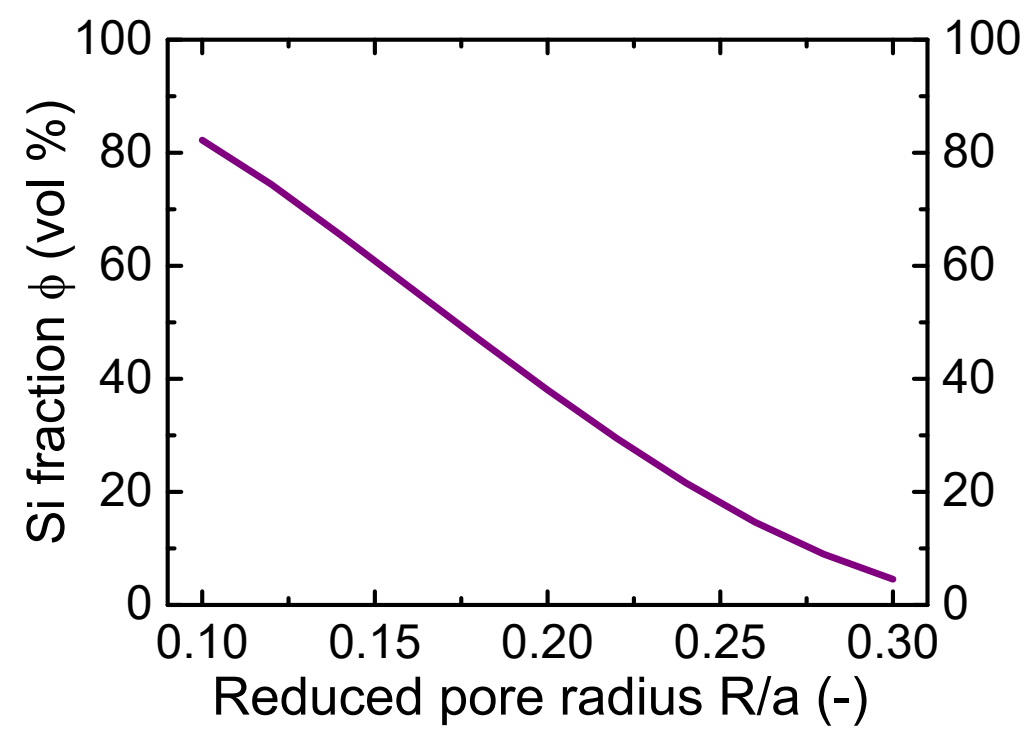

Figure 1.3: Volume fraction of $\operatorname{Si} \phi$ in the inverse woodpile crystal structure as a function of the reduced pore radius $R / a$.

\subsection{Realization and probing of photonic crystals}

It is a challenge to realize $3 \mathrm{D}$ photonic crystal structures with large photonic strength and desired photonic behavior. A large variety of technique has been used for fabrication of 3D photonic nanostructures [35, 51-53]. One first popular way to fabricate 3D nanostructures is the family of template-assisted methods [54-58]. In these methods, one first assembles a template that is infiltrated with a high refractive index material, followed by removal of the template by calcination or etching. A large variety of templates has been demonstrated, such as artificial opals made from colloidal nanoparticles (usually polymer or silica), polymer photoresist structured by 3D holography [59], or resist structured by direct laser writing (DLW) [60-63]. The geometry of the structures fabricated with DLW is well defined and can be very complex such as for example high-quality photonic crystals made with inversion of woodpile templates shown in Figure 1.5(b) [63], where a nearly complete band gap and remarkable optical properties in transmission and reflection were observed. Nevertheless, subsequent inversion to a high refractive index material introduces undesirable yet unavoidable impurities, roughness, and undesired absorption [64].

A second class of impressive structures are layer-by-layer methods [65-68]. An example of a nano structure fabricated by precise micro-manipulation is shown in Figure 1.5(a). Remarkable results have been reported on the fabrication of woodpile photonic crystal structures with layer-by-layer approach $[69,70]$. The main difficulty of layer-by-layer fabrication is the alignment between layers: each 


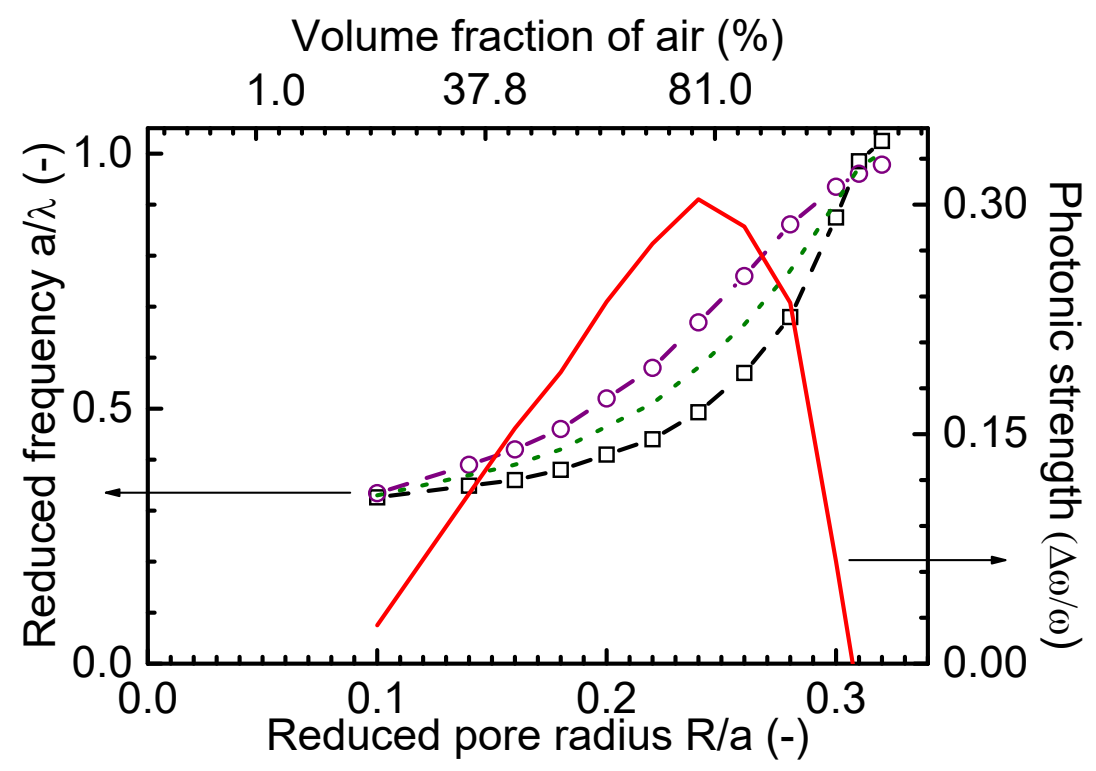

Figure 1.4: Calculated upper (purple) and lower (black) boundaries of the stop gap in $\Gamma X$ direction for the infinite inverse woodpile photonic crystal. The red curve shows the photonic strength $\frac{\Delta \omega}{\omega}$ extracted from the width of the stop gap.
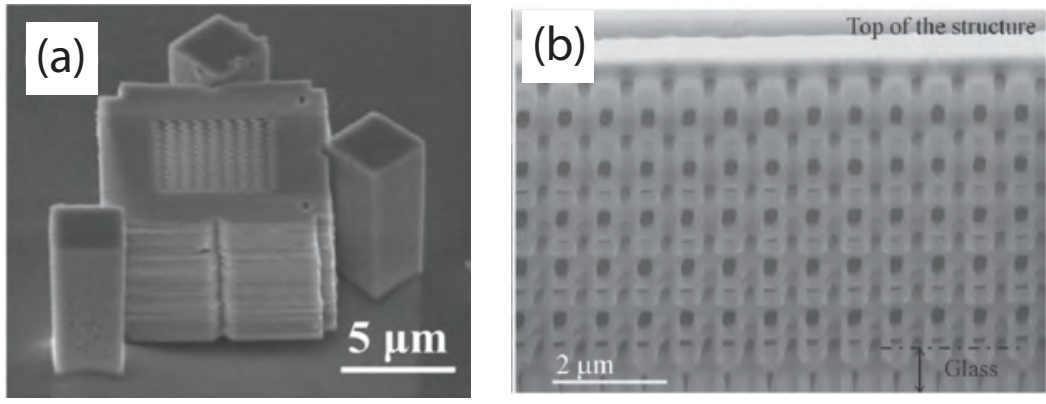

Figure 1.5: (a) Example of the layer-by-layer fabricated structure from Reference [67]. The structure is a $3 \mathrm{D}$ photonic crystal diamond structure made by micro-manipulation in GaAs. The number of layers reaches up to $N=28$. Image courtesy of T. Tajiri and S. Iwamoto. (b) Example of structure fabricated via inversion of woodpile template made by multiphoton lithography [63]. The image is a cross section made by milling under $45^{\circ}$ of a $\mathrm{TiO}_{2}$ partially infiltrated woodpile. Image courtesy of F. Scheffold.

layer has to be carefully aligned with respect to the previous one so no correlation ensured between layer $N$ and $N+1$. Therefore it is debatable whether there is a long range order in $Z$ direction. Here we call such an alignment a planar alignment since the layers that are being aligned are in parallel planes. In complementray metal oxide seimiconductors (CMOS) industry the parameter 
characterizing the planar alignment between two layers is called overlay [71]. For the fabrication of an $N$-layer thick structure $N$-1 alignment steps are needed with $N-1$ overlay requirements.

In order to avoid a large number of alignment steps a third class of fabrication methods has been proposed, where 3D nanostructures are created by consecutively patterning an etch mask on only two adjacent oblique wafer surfaces with only a single alignment step $(n=1)$, followed by deep etching [50,72, 73]. It is important to highlight that in this case the alignment is not planar anymore, as the masks that are aligned with respect to each other are in oblique planes. For the diamond-like photonic crystal under study her, the out-of-plane alignment should be better than $50 \mathrm{~nm}$ [50]. In practice an out-of-plane alignment of 15 $\mathrm{nm}$ was reached [72]. Nevertheless, even one such out-of-plane alignment step introduces a significant complexity in the fabrication procedure, introducing deviations from perfect alignment and extending the time needed for fabrication. In Chapter 2 we introduce a novel single-step etch mask approach that allows to fabricated the 3D etch mask on the oblique surfaces with ensured on the design stage alignment. The approach allows flexible design of nearly arbitrary geometries to be patterned on he inclined surfaces with better than $5 \mathrm{~nm}$ alignment of features located in different planes with respect to each other. We propose design and realize etch mask for 3D inverse woodpile photonic crystal with and without embedded defects discussed in the later chapters. We also investigate the alignment of the resulting etch mask and prove it to be close to resolution of SEM on the example of a mask for 3D hexagonal crystal lattice.

It is important to push nanofabrication beyond academic facilities and provide fabrication solutions for novel structures that are not only interesting for scientific purposes but also for industry. Recent developments in the field of non-volatile memories are already directed towards the 3D integration [74]. One of the most important industrial requirements to nanofabrication is low cost and high throughput. This can be achieved by using wafer scale fabrication of nanostructres with CMOS compatible techniques. Therefore in Chapter $\mathbf{3}$ we propose a possible way to extend the single-step etch mask approach on a wafer scale by means of conventional DUV lithography. The attempts has been made earlier to use highly corrugated surfaces in combination with focused ion beam lithography for nanopatterning such as in the Reference [73]. Nevertheless the minimum feature size achieved in the earlier work stayed in a micrometer scale when the semiconductor industry in steadily moving towards a nanometer feature sizes. In Reference [75] the tilt of the substrate together with the photomask was applied to fabricated 3D microstructures with inclined UV exposure. In this way the microstructures fabricated in the thick layer of negative photoresist first, strongly limited in geometries and second, not transferable into the underlying substrate. Here we propose to employ the conventional UV lithography on the pre-structured silicon substrate to obtain the 3D mask pattern in a single exposure. The substrate is pre-patterned with $\mathrm{V}$-trenches on which photoresist layer is spin coated and DUV exposure is performed. We obtained developed in photoresist features as small as $180 \mathrm{~nm}$ located at different depth on the slope of Si V-trenches. 
After the etch mask is defined the next step in nanofabrication procedure is pattern transfer from the etch mask to the substrate material. The typical requirements for the pattern transfer are high amplification, reproducibility, small roughness and CMOS compatibility. Pattern transfer is done by a large variety of techniques including anisotropic wet etching [76], electrochemical etching [77], dry (plasma) etching [78] and others [79]. While wet etching of Si allows to fabricate impressive structures with the aspect ratio up to 600 it is fundamentally limited in the geometries of fabricated structures as they are defined by the crystal plane orientations [80]. In the approach of electrochemical etching the advantage is that it allows to etch large volumes [77]. The drawback of it is that when the geometry of the structure becomes complex and multiple etching steps are required, the etchant will pour into already existing structure and inevitably destroy it. To achieve both high aspect ratio etching and flexibility in design allows deep reactive ion etching (DRIE). In DRIE the geometry of the feature and etching selectivity controlled by the etch mask [81]. Most of the reported high aspect ratio structures etched by DRIE are trenches with dimensions in nanometer scale in two directions and large extent in third direction. Great difficulties arise when the etched feature is limited in all three directions such as in case of cylindrical pores. Cylindrical pores are important building blocks both for photonics (including 3D inverse woodpile photonic crystals) and CMOS industry. In this thesis we choose DRIE as the most promising type of etching of 3D photonic nanostructures with high aspect ratio. In Chapter 4 we describe the process to etch ultra-high aspect ratio nanopores in Si. The example of the high aspect ratio nanopores etched in Si by DRIE and viewed in the cross section by helium ion microscope with unprecedented resolution is shown in Figure 1.6. The aspect ratio achieved in our experiments reaches up to a world record of 42 . High aspect ratio nanopores are the building blocks for fabrication of $3 \mathrm{D}$ inverse woodpile photonic crystals.

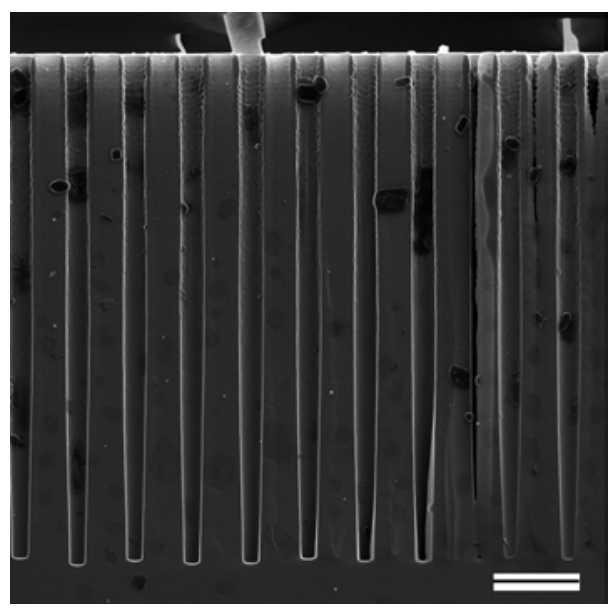

Figure 1.6: High aspect ratio nanopores etching in Si be DRIE and viewed with a helium ion microscope. 1 micron scale bar is shown on the picture. 
The optical properties of photonic nanostructures are in essence determined by their complex internal structure that consist of sub-wavelength-scale 3D arrays (periodic, aperiodic, random) of spheres, rods, pores, split-ring resonators, and other structural units [83-85, 108]. Inevitably, a fabricated structure differs from the initial design, both systematically as with structural deformations $[50,86]$, and statistically as with size polydispersity and random positional disorder $[87,88]$. Consequently, the observed performance differs from the expected one. It is therefore critical to assess the structure of a $3 \mathrm{D}$ material and verify how well it matches the design. The conventional approach is to perform SEM imaging of the fabricated structures [89]. In spite that it is a non-destructive method and provides high resolution images, it only allows to investigate structure from the outside. Moreover, SEM images are generally of a qualitative nature, that is it is difficult to obtain quantitative structural information. Combining the SEM with micromachining or ion beam milling makes it possible to remove a part of the structure and look inside the structure [90]. The disadvantage of such an approach is that the structure under investigation is inevitably irreversibly destroyed. Among the non-destructive methods of materials investigation the family of X-ray methods stands out. A standard X-ray diffraction patterns provide information about microscopic atomic arrangement but information about mesoscopic structure is missing. Structural information at mesoscopic length scale is accessible by small-angle x-ray scattering. Quantitative data on photonic crystals were notably obtained by our group [91, 92]. This brings us to a field of tomography techniques, which provide a three-dimensional image of a sample [93]. In traditional tomography, the contrast is provided by the sample absorption that is simply related to the brightness of the transmitted image or radiograph. However, since silicon and materials that prevail in nanophotonics and CMOS industry are weakly absorbing, other tomography methods are required. In the Chapter $\mathbf{5}$ of this thesis we obtain real space structural information directly from the optical phase change of an X-ray beam that propagates through the sample and that depends on the real part of the refractive index. The phase change is quantitatively retrieved from a set of radiographs taken at multiple sample-to-detector distances while rotating the sample (see Methods) [94]. Following a conventional tomographic reconstruction of the retrieved phase maps, the $3 \mathrm{D}$ electron density $\rho_{e}(X, Y, Z)$ is obtained in real space. To achieve nanometer spatial resolution, projection microscopy or zoom tomography is employed $[95,96]$. Its main features are that the X-ray beam is focused, and that the sample is placed at a small distance $z_{s}$ downstream from the focus to collect magnified Fresnel diffraction patterns on the detector [97, 98]. We consider several phase retrieval algorithms and experimental settings to achieve the best reconstruction for our kind of samples. From tomography data we extract important parameters of 3D photonic crystals such as pore radii, pore depth, mutual alignment of the pores and $R / a$ ratio.

Once photonic structure is realized and verified to be matching the design it is time to discover its optical performance. Depending on the functionality of the structure different experiments serve to evaluate its performance [99-101]. The most intriguing feature of $3 \mathrm{D}$ photonic crystals is the existence of a $3 \mathrm{D}$ photonic 
band gap - a range of frequencies for which light is forbidden to propagate in any direction and for any polarization. In the ideal case of an infinite photonic crystal the density of optical states (DOS) as well as local density of states (LDOS) are zero in the band gap [42, 102-104]. In reality though the actual fabricated samples are always finite size and their optical properties differ from the infinite size crystal. Thus it was shown that the LDOS in the band gap of the finite size crystal are non-zero and depend on frequency, position and crystal size [105-107]. Multiple different approaches are used to verify the presence of the complete band gap in a crystal. One method is to probe the LDOS and thus verify the presence of complete photonic band gap is emission measurements since the radiative decay rate of emitters is proportional to LDOS $[100,101]$. The accuracy of this method depends strongly on the precision with which the emitter is placed inside the crystal and quantum efficiency of the emitters. Another method is to measure reflected or transmitted light from photonic structure. In the case of stop gap, light with incident $k$ vectors satisfying Bragg condition will be reflected as it cannot propagate inside the crystal. In case of a complete photonic band gap all incident $k$ vectors will be reflected back and in all polarizations. To investigate the photonic behavior of our samples in the Chapter 6 we choose to perform broadband microscopy reflectivity measurements on fabricated structures. We also study the intriguing polarization behavior of the parent $2 \mathrm{D}$ structures of $3 \mathrm{D}$ inverse woodpiles by systematically rotating polarization and keeping analyzer parallel or perpendicular to polarizer. 



\section{Bibliography}

[1] C. Hyugens (translated by S.P. Thompson, 1912) Treatise on Light Project Gutenberg (2005) 9

[2] Euclid Optica, (The English translation is available as: H. E.Burton, The Optics of Euclid), J. Opt. Soc. Am. 255 (1945) 9

[3] S.H. Yun and S.J.J. Kwok Light in diagnosis, therapy and surgery, Nat. Biomed. Eng. 1, 0008 (2017) 9

[4] Q. Chen, X. Hu, L. Wen, Y. Yu, and D. R.S. Cumming Nanophotonic Image Sensors, Small 12, 4922-4935 (2016) 9

[5] M.C. Estevez, M. Alvarez, L.M. Lechuga Integrated optical devices for labon-a-chip biosensing applications Laser Photon. Rev. 6, 463-487 (2012) 9

[6] F. Vollmer and S. Arnold Whispering-gallery-mode biosensing: label-free detection down to single molecules, Nature Methods 5, 591-596 (2008) 9

[7] M. Loncar Molecular sensors: Cavities lead the way, Nature Phot. 1, 565-567 (2007) 9

[8] A. Einstein Zur Elektrodynamik bewegter Krper, Annalen der Physik 17891 (1905) 9

[9] P.A. Clayton, and M.J. Price The Seven Wonders of the Ancient World, Routledge, Abingdon (1998) 9

[10] G.T. Reed Device physics: The optical age of silicon, Nature 427, 595-596 (2004) 9

[11] R.A. Soref and B.R. Bennett Electrooptical effects in silicon, IEEE J. Quant. Electron. QE-23, 123129 (1987) 9

[12] M. Snit, J. van der Tol, and M. Hill Moore's law in photonics Laser Photon. Rev. 6, 1-3 (2012) 9

[13] J. Orton The story of semiconductors Oxford University Press (2004) 9

[14] R.A. Soref Silicon-Based Optoelectronics, Proc. of IEEE 81, 1687-1706 (1993) 9

[15] L.Chrostowski, M. Hochberg Silicon photonics design: from devices to systems, Cambridge University Press (2015) 9

[16] C.K. Tang and G.T. Reed Highly efficient optical phase modulator in SOI waveguides, Electron. Lett. 31, 451-452 (1995) 9

[17] A. Liu, R. Jones, L. Liao, D. Samara-Rubio, D. Rubin, O.Cohen, R. Nicolaescu, and M. Paniccia A high-speed silicon optical modulator based on a metaloxidesemiconductor capacitor, Nature 427, 615-618 (2004) 9

[18] G.T. Reed, G. Mashanovich, F.Y. Gardes, and D.J. Thomson Silicon optical modulators, Nature Photon. 4, 518-526 (2010) 10

[19] P.W. Coteus, J.U. Knickerbocker, C.H. Lam, Y. A. Vlasov Technologies for 
exascale systems IBM J. Res. \& Dev. 5514 (2011) 10

[20] D.A. B. Miller Device Requirements for Optical Interconnects to Silicon Chips Proc. of IEEE 97, 1166-1185 (2009) 10

[21] Yu. Vlasov Silicon CMOS-Integrated Nano-Photonics for Computer and Data Communications Beyond 100G IEEE Comm. Mag. 12, S67-S71 (2012) 10

[22] J. Leuthold, C. Koos, and W. Freude Nonlinear silicon photonics Nature Photon. 4, 535-544 (2010) 10

[23] https://www.micron.com/about/our-innovation/3d-xpoint-technology Micron press release 17.04.2017 10

[24] http://www.samsung.com/semiconductor/products/flash-storage/v-nand/

Samsung brochure 20.06.2016 10

[25] E. Yablonovitch Inhibited spontaneous emission in solid-state physics and electronics Phys. Rev. Lett. 58, 2059-2062 (1987) 10

[26] O. S. Ojambati Stirring the propagation and the absorption of light in complex nanophotonic systems $\mathrm{PhD}$ thesis (2016) 10

[27] A. P. Mosk, A. Lagendijk, G. Lerosey, and M. Fink Controlling waves in space and time for imaging and focusing in complex media (Review) Nature Photon. 6, 283-292 (2012) 10

[28] D.S. Wiersma Disordered photonics Nat. Phot. 7 188-196 (2013) 10

[29] S. John Strong localization of photons in certain disordered dielectric superlattices Phys. Rev. Lett. 58, 2486-2489 (1987) 10, 11

[30] K. Ishizaki, M. Koumura, K. Suzuki, K. Gondaria, and S. Noda Realization of three-dimensional guiding of photons in photonic crystals Nature Photon. 7133-137 (2013) 10

[31] W.L. Bragg Diffraction of short electromagnetic waves by a crystal, Proc. Camb. Phil. Soc. 17, 43 (1913) 10

[32] C. Kittel Introduction to Solid State Physics John Wiley \& Sons (2005) 11

[33] S. John Quantum electrodynamics of localized light Physica B: Phys. of Cond. Matt. 175 87-95 (1991) 11

[34] S. John Frozen light Nature 390, 661-662 (1997) 11

[35] M. Ghulinyan and L. Pavesi L (eds) Vos W and Woldering L ch 8 Cavity quantum electrodinamics with three-dimensional photonic band gap crystals (Cambridge: Cambridge University Press) (2015) 11, 14

[36] S. Haroche Cavity quantum electrodynamics: Fundamental systems in quantum optics North Holland, Amsterdam (1992) 11

[37] J. G. Fleming, S. Y. Lin, I. El-Kady, R. Biswas, and K. M. Ho, All-metallic three-dimensional photonic crystals with a large infrared bandgap, Nature 417, $52(2002) 11$

[38] C. Blum, N. Zijlstra, A. Lagendijk, M. Wubs, A. P. Mosk, V. Subramaniam, and W. L. Vos Nanophotonic control of the forster resonance energy transfer efficiency, Phys. Rev. Lett. 109, 203601 (2012) 11

[39] G. Kurizki and A. Genack Suppression of molecular interactions in periodic dielectric structures, Phys. Rev. Lett. 61, 2269 (1988) 11

[40] M. I. Antonoyiannakis and J. B. Pendry Electromagnetic forces in photonic crystals, Phys. Rev. B 60, 2363 (1999) 11 
[41] W.H. Zurek Decoherence and the transition from quantum to classical Phys. Today 44, 36 (1991) 11

[42] J.D. Joannopoulos, S.G. Johnson, J.N. Winn, and R. D. Meade Photonic crystals: molding the flow of light Princeton university press (2008) 11, 19

[43] L. A. Woldering, A. P. Mosk, and W. L. Vos Design of a 3D photonic band gap cavity in a diamond-like inverse woodpile photonic crystal Phys. Rev. B 90, 115140: 1-9 (2014) 11

[44] W. L. Vos, R. Sprik, A. van Blaaderen, A. Imhof, A. Lagendijk, and G. H. Wegdam, Strong effects of photonic band structures on the diffraction of colloidal crystals, Phys. Rev. B 53, 16231 (1996) 11

[45] M. Megens Structure and fluorescence of photonic colloidal crystals Ph.D. thesis, University of Amsterdam (1999) 11

[46] K. M. Ho, C. T. Chan, C. M. Soukoulis, R. Biswas, and M. Sigalas Photonic band gaps in three dimensions: new layer-by-layer periodic structures, Solid State Comm. 89, 413 (1994) 13

[47] M. Maldovan and E. L. Thomas Diamond-structured photonic crystals, Nature Mat. 3, 593 (2004) 13

[48] A. Chutinan and S. Noda Spiral three-dimensional photonic band-gap structure, Phys. Rev. B 57, R2006 (1998) 13

[49] R. Hillebrand, S. Senz, W. Hergert, and U. G'osele Macroporous siliconbased three-dimensional photonic crystal with a large complete band gap, J. Appl. Phys. 94, 2758 (2003) 13

[50] L.A. Woldering, A.P. Mosk, R.W. Tjerkstra, and W.L. Vos, The influence of fabrication deviations on the photonic band gap of three-dimensional inverse woodpile nanostructures, J. Appl. Phys. 105, 093108: 1-10 (2009). 13, 16, 18

[51] C. López Materials Aspects of Photonic Crystals, Adv. Mater 15, 1679-1704 (2003) 14

[52] J.F. Galisteo-López, M. Ibisate, R. Sapienza, L.S. Froufe-Pérez, Á. Blanco , and C. López Self-Assembled Photonic Structures, Adv. Mater 23, 30-69 (2010) 14

[53] M. Ghulinyan, and L. Pavesi (eds) Light Localisation and Lasing: Random and Quasi-Random Photonic Structures (Cambridge: Cambridge University Press) (2015) 14

[54] Yu. A. Vlasov, B. Xiang-Zheng, C.S. James, and D.J. Norris On-chip natural assembly of silicon photonic bandgap crystals, Nature 414, 289-293 (2001) 14

[55] P. Braun Materials Chemistry in 3D Templates for Functional Photonics Chem. Mater 26, 277-286 (2014) 14

[56] A. Imhof, D.J. Pine Ordered macroporous materials by emulsion templating, Nature 389, 948-951 (1997) 14

[57] B.T. Holland, C.F. Blanford, and A. Stein Synthesis of macroporous minerals with highly ordered three-dimensional arrays of spheroidal voids, Science 281, 538-540 (1998) 14

[58] J.E.G.J. Wijnhoven and W.L. Vos 1998 Preparation of photonic crystals made of air spheres in titania, Science 281, 802-804 (1998) 14

[59] M. Campbell, D.N. Sharp, M.T. Harrison, R.G. Denning, and A.J. Turberfield Fabrication of photonic crystals for the visible spectrum by holographic 
lithography, Nature 404, 53-56 (2000) 14

[60] H.B. Sun, S. Matsuo, H. Misawa Three-dimensional photonic structures archieved with two-photon-absorption photopolymerization of resin Appl. Phys. Lett 74, 786-788 (1999) 14

[61] B.H. Cumpston, S.P. Ananthavel, S. Barlow, D.L. Dyer, J.E. Ehrlich, L.L. Erskine, A.A. Heikal, S.M. Kuebler, I.Y. Sandy Lee, D. McCord-Maughon, J. Qin, H. Rckel, M. Rumi, X.L. Wu, S.R. Marder, and J.W. Perry Twophoton polymerization initiators for three-dimensional optical data storage and microfabrication Nature, 398 51-54 (1999) 14

[62] M. Deubel, M. Wegener, A. Kasao, and S. John Direct laser writing and characterization of Slanted Pore Photonic Crystals, Appl. Phys. Lett, 85 18951897 (2004) 14

[63] C. Marichy, N. Muller, L.S. Froufe-Pérez, and F. Scheffold, High-quality photonic crystals with a nearly complete band gap obtained by direct inversion of woodpile templates with titanium dioxide, Sci. Rep. 6, 21818: 1-8 (2015) 14, 15

[64] J.E.G.J. Wijnhoven, L. Bechger, and W.L. Vos Fabrication and Characterization of Large Macroporous Photonic Crystals in Titania, Chem. Mater. 13, 4486-4499 (2001) 14

[65] J.G. Fleming and S.Y. Lin Three-dimensional photonic crystal with a stop band from 1.35 to $1.95 \mathrm{~m}$, Opt. Lett. 24, 49-51 (1999) 14

[66] S. Ogawa, M. Imada, S. Yoshimoto, M. Okano, and S. Noda 2004 Control of light emission by 3D photonic crystals, Science 305, 227-229 (2004) 14

[67] T. Tajiri, S. Takahashi, Y. Ota, J. Tatebayashi, S. Iwamoto, and Y. Arakawa Demonstration of a three-dimensional photonic crystal nanocavity in a 110layered diamond structure, Appl. Phys. Lett. 107, 071102 (2015) 14, 15

[68] S. Iwamoto, S. Takahashi, T. Tajiri, and Y.Arakawa Semiconductor ThreeDimensional Photonic Crystals with Novel Layer-by-Layer Structures, Photonics 34, 34 (2016) 14

[69] S. Ogawa, K. Ishizaki, T. Furukawa, and S. Noda Spontaneous emission control by 17 layers of three-dimensional photonic crystals, Electronics Lett. 44, 377-378 (2008) 14

[70] S. Noda, K. Tomoda, N. Yamamoto, and A. Chutinan Full three-dimensional photonic bandgap crystals at near-infrared wavelengths, Science 289, 604606 (2000) 14

[71] H.J. Levinson Principles of Lithography (Bellingham: SPIE Press) p 363 (2001) 16

[72] R.W. Tjerkstra, L.A. Woldering, J.M. van den Broek, F. Roozeboom, I.D. Setija, and W.L. Vos A method to pattern masks in two inclined planes for three-dimensional nano- and microfabrication J. Vac. Sci. Technol. B 29, 061604: 1-8 (2011) 16

[73] M. Erdmanis, P. Sievil, A. Shah, N. Chekurov, V. Ovchinnikov, and I. Tittonen Focused ion beam lithography for fabrication of suspended nanostructures on highly corrugated surfaces Nanotechnology 25, 335302 (2014) 16

[74] Y. Fujisaki Review of Emerging New Solid-State Non-Volatile Memories Jpn. J. Appl. Phys. 52, 040001:1-11 (2013) 16 
[75] M. Han, W. Lee, S.-K. Lee, S.S. Lee $3 D$ microfabrication with inclined/rotated UV lithography Sens. and Act. A: Phys. 111, 14-20 (2004) 16

[76] K. E. Bean Anisotropic etching of silicon, IEEE Transactions of Electron Devices 25, 1185 (1978) 17

[77] J. E. A. M. van den Meerakker, R. J. G. Elfrink, F. Roozeboom, and J. F. C. M. Verhoeven Etching of deep macropores in 6in. Si wafers, J. Electrochem. Soc. 147, 2757 (2000) 17

[78] F. Marty, L. Rousseau, B. Saadany, B. Mercier, O. Francais, Y. Mita, and T. Bourouina Advanced etching of silicon based on deep reactive ion etching for silicon high aspect ratio microstructures and threedimensional micro- and nanostructures, Microelectronics Journal 36, 673 (2005) 17

[79] Y.Nishi and R.Doering Handbook of Semiconductor Manufacturing Technology Marcel Dekker. New York, (2000) 17

[80] B. Wu, A. Kumar, and S. Pamarthy High aspect ratio silicon etch: A review J. Appl. Phys. 108, 051101 (2010) 17

[81] A. A. Ayón, K. S. Chen, K. A. Lohner, S. M. Spearing, H. H. Sawin, and M. A. Schmidt Deep reactive ion etching of silicon Mater. Res. Soc. Symp. Proc., vol. 546, pp. 5161, 199917

[82] C.M. Soukoulis, M. Wegener, Nature Photon 5, 523 (2011) 18

[83] J.K. Gansel et al., Science 325, 1513 (2009) 18

[84] J.E.G.J. Wijnhoven, W.L. Vos, Science 281, 802 (1998) 18

[85] S. Noda, K. Tomoda, N. Yamamoto, A. Chutinan, Science 289, 604 (2000) 18

[86] S.H. Fan, P.R. Villeneuve, J.D. Joannopoulos, J. Appl. Phys 78, 1415 (1995). 18

[87] S. Hughes, L. Ramunno, J.F. Young, J.E. Sipe, Phys. Rev. Lett. 94, 033903 (2005) 18

[88] A.F. Koenderink, A. Lagendijk, W.L. Vos, Phys. Rev. B 72, 053102 (2005). 18

[89] D. McMullan Scanning electron microscopy Science 17, 175-185 (1995) 18

[90] J. Goldstein, D.E. Newbury, D.C. Joy, C.E. Lyman, P. Echlin, E. Lifshim, L. Sawyer, and J.R. Michael Scanning Electron Microscopy and X-ray Microanalysis (Springer science + business media, New York, 2003) 3rd Edition 18

[91] J. E. G. J. Wijnhoven, L. Bechger, and W. L. Vos Fabrication and characterization of large macroporous photonic crystals in titania Chem. Mater. 13 4486-4499 (2001) 18

[92] M. Megens, and W. L. Vos Particle excursions in colloidal crystals Phys. Rev. Lett. 86, 4855-4858 (2001) 18

[93] B. Pollak Experiences with Planography Dis Chest 24, 663-669 (1953) 18

[94] S. Zabler, P. Cloetens, J.-P. Guigay, J. Baruchel, and M. Schlenker, Optimization of phase contrast imaging using hard $x$ rays Rev. Sci. Instrum. 76, 073705:1-7 (2005) 18

[95] C. S. Schroer, J. Meyer, M. Kuhlmann, B. Benner, T. F. Gunler, B. Lengeler, C. Rau, T. Weitkamp, A. Snigirev, and I. Snigireva, Nanotomography based on hard $x$-ray microscopy with refractive lenses Appl. Phys. Lett. 81, 1527-1529 
(2002) 18

[96] C. A. Larabell and M. A. L. Gros, X-ray Tomography Generates 3-D Reconstructions of the Yeast, Saccharomyces cerevisiae, at 60-nm Resolution Mol. Biol. Cell 15, 957-962 (2003) 18

[97] R. Mokso, P. Cloetens, E. Maire, W. Ludwig, and J.-Y. Buffire, Nanoscale zoom tomography with hard $x$ rays using Kirkpatrick-Baez optics Appl. Phys. Lett. 90, 144104 (2007) 18

[98] P. Cloetens, W. Ludwig, J. Baruchel, D. van Dyck, J. van Landuyt, J.P. Guigay, and M. Schelenker, Holotomography: Quantative phase tomography with micrometer resolution using hard synchrotron radiation $x$ rays, Appl. Phys. Lett. 75, 2912 (1999) 18

[99] S. Takayama, H. Kitagawa, Y. Tanaka, T. Asano, and S. Noda, Experimental demonstration of complete photonic band gap in two-dimensional photonic crystal slabs Appl. Phys. Lett. 87, 061107 (2005) 18

[100] A. F. Koenderink, L. Bechger, A. Lagendijk, and W. L. Vos, An experimental study of strongly modified emission in inverse opal photonic crystals Phys. Stat. Sol. (A) 197, (2003) 648-661 18, 19

[101] P. Lodahl, A. F. van Driel, I. S. Nikolaev, A. Irman, K. Overgaag, D. Vanmaekelbergh, and W. L. Vos, Controlling the dynamics of spontaneous emission from quantum dots by photonic crystals Nature 430, (2004) 654-657 18,19

[102] Z.Y. Li and Y. Xia Optical photonic band gaps and the Lamb shift, Phys. Rev. A, 63, 043817 (2001) 19

[103] K. Bush and S. John Photonic band gap formation in certain self-organizing systems, Phys. Rev. E, 58, 3896 (1998) 19

[104] C.M. Soukoulis Photonic band gap materials: "semiconductors" of the future?, Phys. Scr. T 66, (1996) 146-150 19

[105] M. D. Leistikow, A. P. Mosk, E. Yeganegi, S. R. Huisman, A. Lagendijk, and W. L. Vos Inhibited spontaneous emission of quantum dots observed in a $3 D$ photonic band gap, Phys. Rev. Lett. 107, 193903: 1-5 (2011) 19

[106] D. P. Fussell, R. C. McPhedran, and C. Martijn de Sterke Threedimensional Greens tensor, local density of states, and spontaneous emission in finite two-dimensional photonic crystals composed of cylinders, Phys. Rev. E 70, 06608 (2004) 19

[107] E. Yeganegi, A. Lagendijk, A.P. Mosk, and W.L. Vos Local density of optical states in the band gap of a finite one-dimensional photonic crystal Phys. Rev. B 89, (2014) 045123: 1-10 19

[108] C.M. Soukoulis, and M. Wegener, Past achievements and future challenges in the development of three-dimensional photonic metamaterials, Nature Photon 5, 523 (2011) 18 


\section{CHAPTER 2}

\section{Single-step etch mask for 3D monolithic}

\section{nanostructures}

We present a method to fabricate a 3D mask that allows to etch three-dimensional monolithic nanostructures by using only CMOScompatible processes. The mask is written in a hard-mask layer that is deposited on two adjacent inclined planes of a Si wafer. By projecting in a single step two different $2 \mathrm{D}$ patterns within one $3 \mathrm{D}$ mask on the two inclined planes, the mutual alignment between the patterns is ensured. After the mask pattern is defined, the etching of deep pores in two oblique directions yields a three-dimensional structure in Si. As a proof of concept we demonstrate 3D mask fabrication for threedimensional diamond-like photonic band gap crystals in silicon. The fabricated crystals reveal a broad stop gap in optical reflectivity measurements. We propose how 3D nanostructures with five different Bravais lattices can be realised, namely cubic, tetragonal, orthorhombic, monoclinic, and hexagonal, and demonstrate a mask for a 3D hexagonal crystal. We also demonstrate the mask for a diamond-structure crystal with a $3 \mathrm{D}$ array of cavities. In general, the $2 \mathrm{D}$ patterns on the different planes can be completely independently structured and still be in perfect mutual alignment. Indeed, we observe an alignment accuracy of better than $3.0 \mathrm{~nm}$ between the $2 \mathrm{D}$ mask patterns on the inclined planes, which permits one to etch well-defined monolithic 3D nanostructures.

\subsection{Introduction}

In this chapter we present a new fabrication method for monolithic 3D nanostructures. The key step is to define and make a single step etch mask on two inclined planes simultaneously with an out-of-plane alignment ensured at the design stage. As an example for a single step etch mask, we realise a pattern that yields a (110) plane of a cubic inverse woodpile structure on one surface and (11̄0) plane on a perpendicular adjacent surface. We describe the fabrication process of such a so-called "3D mask", as well as the subsequent etching process to fabricate 3D photonic band gap crystals with diamond-like inverse woodpile structure (see Figure 2.1). We investigate the ensured alignment at the design stage by 
carefully characterizing the realised structures on the inclined planes. We study nanophotonic behaviour of the fabricated crystal by reflectivity measurements. We discuss other 3D structures that are feasible using our method such as disordered structures and different 3D Bravais lattices; as a proof of principle, we demonstrate a 3D mask for a hexagonal 3D nanostructure, and a 3D cavity array. Finally we discuss the use of various lithography techniques with our method.

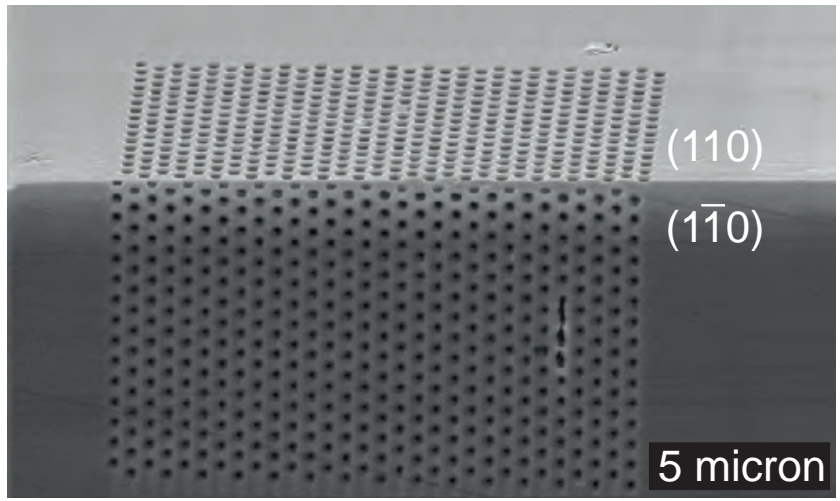

Figure 2.1: SEM image of a monolithic 3D photonic band gap crystal fabricated in Si using a single step etch mask. The crystal has the inverse woodpile structure with a cubic diamond-like symmetry that consists of two sets of perpendicular pores. The top surface in the image is the (110) crystal plane and the perpendicular surface at the bottom is the $(1 \overline{1} 0)$ crystal plane. The scale bar is shown in the image. The typical radius of the pore is $R=160 \mathrm{~nm}$ and the lattice constant $a=680 \mathrm{~nm}$.

\subsection{Fabrication process for the 3D single-step etch mask with built-in alignment}

The generic scheme to fabricate a 3D etch mask on two inclined planes is shown in Figure 2.2. First, the hard mask material that serves as an etch stop is deposited on two inclined planes, see Figure 2.2(a). Next, the desired pattern is written onto the oblique surfaces, as shown in Figure 2.2(b). The projection is made from a side such that both inclined planes can be reached. The projected pattern consists of two parts: pattern $a$, designed for one surface and pattern $b$, designed for the second surface. We emphasize that our method allows for a complete freedom to independently design the two patterns $a$ and $b$. In Figure 2.2(b) the two patterns are designed to be similar and in Figure 2.7(b) and (c) examples are given where patterns on oblique planes are designed to be different. The two patterns are written in one projection and are therefore by design in perfect mutual alignment. Mask apertures for subsequent etching are opened during the third step of the mask fabrication process (Figure 2.2(c)).

The fact that the pattern is projected on a non-normal surface must be taken into account in the pattern design. The pattern is designed in such a way that 


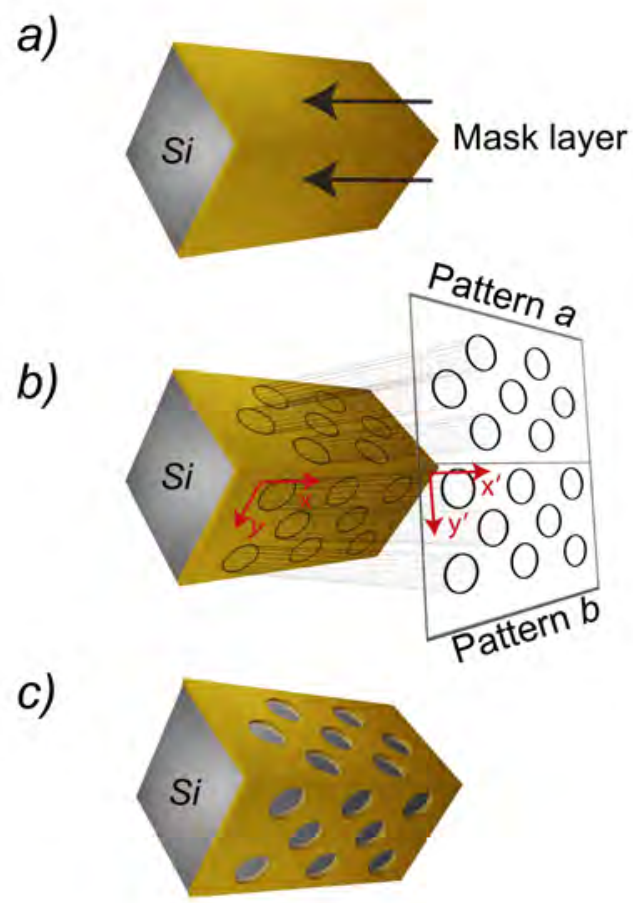

Figure 2.2: Scheme for the single step etch mask fabrication on two perpendicularly inclined planes. a) Deposition of a hard mask layer on two inclined planes of a Si wafer. b) Patterning of mask layer in one step on both planes - projection of single 2D mask on 3D surface. Patterns for both perpendicular planes are written in one projection and therefore alignment is ensured. c) Apertures are opened in the mask layer to obtain an etch mask for two intersecting 2D structures that yield the desired 3D structure.

after projection on the inclined planes it yields the desired structure. As is shown in Figure 2.2(b), the $x^{\prime}$ and $y^{\prime}$ coordinates in the design plane differ from the $x$ and $y$ coordinates on the sample surface.

We demonstrate our fabrication process using as an example a 3D cubic diamondlike photonic band gap crystal made from silicon. Due to its physical properties silicon plays an important role both in optics and electronics. As a material that is widely used in research and manufacturing, silicon is widely available, cheap, and has a very high purity. Among the diamond-like photonic band gap structures [2] we chose the inverse-woodpile photonic crystal. Inverse-woodpile photonic crystals deserve particular attention in view of the broad band gap with relative width $\left(\Delta \omega / \omega_{c}\right)$ of more than $25 \%[1,3,4]$. These crystals consist of two mutually perpendicular rectangular arrays of cylindrical pores etched in a high refractive index material. Conceptually the fabrication of an inverse-woodpile structure is easy, although its fabrication remains a challenge due to the required 


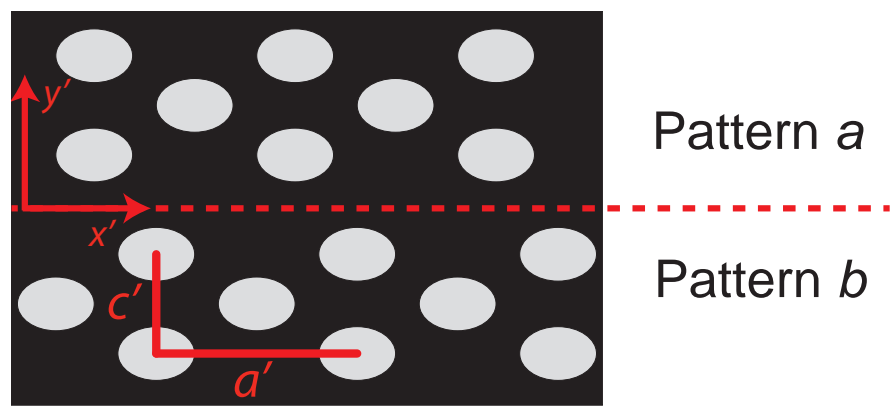

Figure 2.3: Geometry of the pattern that is projected on two inclined planes. The structure is designed to consist of two centred rectangular arrays shifted with respect to each other by $x^{\prime}=c^{\prime} / 4$ such that after being projected on two $45^{\circ}$ inclined surfaces it gives the (110) and (110) faces of a cubic inverse woodpile photonic crystal with lattice parameters $a=\sin 45^{\circ} \times a^{\prime}$ and $c=c^{\prime}$. The basic building blocks are designed to be elliptical, in order to yield circles on the mask after projection. The dashed line delimits the top part of the pattern (pattern $a$ ) that is projected on one surface of the wafer and the bottom part (pattern $b$ ) that is projected on the second surface.

precise alignment of the perpendicular sets of pores $[5,6,24]$.

We start the fabrication procedure from a single crystalline $\mathrm{Si}$ wafer. We fabricated the 3D etch mask on two polished adjacent perpendicular surfaces of the wafer. In the first step we deposited a $50 \mathrm{~nm}$ thick Cr layer that serves as a hard mask material on two adjacent planes of a wafer (Figure 2.2(a)). We choose $\mathrm{Cr}$ as a hard mask material due to its sustainability to $\mathrm{SH}_{6}$ etching [7], but other possible mask materials such as $\mathrm{SiN}$ or $\mathrm{SiC}$ are also compatible with our method. The deposition of $\mathrm{Cr}$ is done in a home-built sputtering machine and takes around 4 minutes for a $50 \mathrm{~nm}$ thick layer.

The patterning of the etch mask can be performed using several types of lithography [8] such as e-beam, focused ion beam (FIB), deep UV (DUV) step-and-scan, or nanoimprint. Since the patterning of the etch mask is performed under an angle $\theta$, the depth of focus $F$ of a lithography tool will limit the mask size along the $y$-direction (Figure 2.2(b)) to $\Delta y=F / \sin \theta$. E-beam lithography tools and FIB may be refocused during patterning and thus the depth of focus can be extended to the desired value $[9,10]$. For DUV lithography the depth of focus (DOF) and the lateral resolution that is also called critical dimension (CD) depend on the wavelength of UV source $\lambda$, numerical aperture of the illumination lens $N A$ and technological factors $k_{1}, k_{2}: \mathrm{CD}=k_{1} \frac{\lambda}{N A}, \mathrm{DOF}=k_{2} \frac{\lambda}{N A^{2}}$. For photonic applications considered here the length scales are in the order of the wavelength of light which is much larger (about $20 \times$ ) than typical length scale in electronic integrated circuits and therefore more than a micron-sized depth of focus may be expected. In case of nanoimprint lithography (NIL) the stamp can be fabricated using e-beam lithography and later transferred into a conformal mask pattern as described for example in Ref. [11]. In our case we had focused ion beam (FIB) milling equipment at our disposition to project the mask and open the apertures. We placed a sample in a FEI Nova 600 Nanolab FIB chamber under $45^{\circ}$ angle 
with respect to the ion beam gun so that both adjacent planes of the wafer can be reached (Figure $2.2 \mathrm{~b}$ ).

The design of a single pattern that is projected on two adjacent planes consists of two parts as shown in Figure 2.3: pattern $a$ intended for one plane and pattern $b$ intended for the second plane. In our case the planes are orthogonal to each other and aligned at $45^{\circ}$ angle with respect to the ion beam gun in $y$ direction (Figure 2.2(b)). In the pattern design it is taken into account that projection of the pattern is made under an angle $\theta=45^{\circ}$ to the planes, meaning that $x^{\prime}$ and $y^{\prime}$ coordinates in the pattern design are related to the $x$ and $y$ coordinates on a surface of fabricated mask as following: $x^{\prime}=x$ and $y^{\prime}=y / \sin \theta$. In order to form a cubic diamond-like structure inside the silicon wafer we patterned each plane of the wafer with a centred rectangular array of holes with lattice parameters $a$ and $c$, where $\frac{a}{c}=\sqrt{2}$ to fulfil the criterion for a cubic crystal. Arrays of holes for two surfaces are shifted by $c / 4$ in the $x$-direction. The pattern is shown in Figure 2.3 and yields (110) and (110) crystal planes on a surface of a wafer. The mask pattern is projected on both planes in one step. Since the two patterns intended for different planes are contained in one image, the alignment between them is ensured. A patterning of a 3D mask consisting of two arrays of 30 by 30 holes each takes 7 minutes.

After the etch mask is created, the next step is to etch deep pores inside $\mathrm{Si}$ through the openings. Etching can be done using a variety of techniques [12] such as reactive ion etching (RIE) [13], cryogenic etching, wet etching, photoelectrochemical etching $[14,15]$, or other types of etching depending on a desired structure ans mask material. Nanopores are first etched in one direction, then the sample is rotated by $90^{\circ}$ and pores are etched in the second perpendicular direction. We etched deep nanopores using deep reactive ion etcher (DRIE) Adixen AMS 100SE as described in reference [13]. It has been shown earlier that the air-silicon interface deflects ions by only a small angle [6]. Moreover for diamondlike inverse woodpile 3D photonic crystal it has been shown that its band gap is robust to deviations of the pore directions [16]: even with misalignments as large as $5^{\circ}$, the relative band gap width is only reduced from $24 \%$ to $21 \%$. Since we etch both sets of pores one after another we introduce an additional oxygen plasma cleaning step after etching each set of pores. The cleaning step is needed to remove the protective polymer layer remaining after the etching process into the front surface of the sample.

\subsection{Results and discussion}

\subsubsection{Fabricated structure characterization}

The result of patterning a 3D etch mask in a single step is shown in Figure 2.4. Figure 2.4(a) shows four 3D masks in a row on the edge of a Si wafer. Each of the $3 \mathrm{D}$ masks was written in one step and consists of (110) and (110) crystal planes of the cubic inverse woodpile crystal. The number of 3D mask structures written along the edge of a Si wafer is only limited by the size of the wafer. The maximum width in the $x$-direction of a single structure that is written in one 
step is determined by the horizontal field of view of the lithography tool which is in our case a FIB setup. In this study the horizontal field of view was $12.8 \mu \mathrm{m}$ as set by the magnification of $10000 \times$. By decreasing the magnification or stitching the fields of view, it is possible to increase the size of a continuous structure in the $x$ direction. Thus, we may effectively consider the four closely spaced 3D patterns in Figure 2.4(a) as one large $L_{x}=40 \mu \mathrm{m}$ sized nanostructure. The size in the $y$-direction is limited by the depth of focus of the tool and can be extended by performing for example multiple milling runs at different depths.

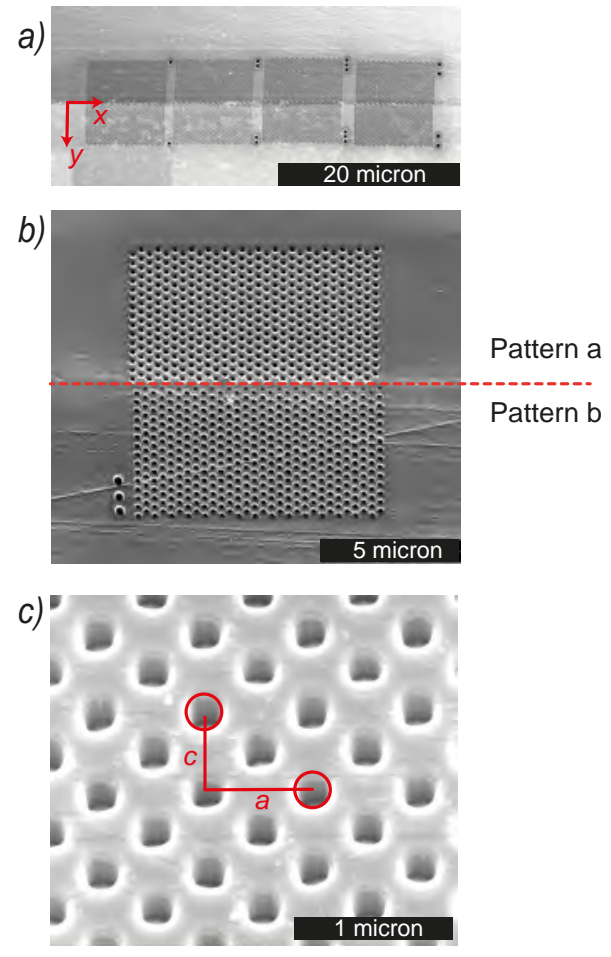

Figure 2.4: a) Overview of a $\mathrm{Si}$ wafer with four $3 \mathrm{D}$ etch masks milled in one step. The coordinates $(x, y)$ are indicated. b) Side view on one of the mask patterns. The dashed line in the middle indicates the 90 degree edge of the Si wafer. c) Zoom-in on one surface of a mask pattern. $a$ and $c$ are lattice parameters with $\frac{a}{c}=\sqrt{2}$. Scale bars are shown in each image.

Figure 2.4(b) shows one complete mask patterned on both wafer planes. Above the dashed red line there is a surface that contains pattern $a$ corresponding to a (110) crystal plane of the targeted photonic crystal. Below the dashed line there is a perpendicular surface that contains pattern $b$ corresponding to a $(1 \overline{1} 0)$ crystal plane. The design for this mask shown in Figure 2.3 is such that centres of the apertures in pattern $b$ are shifted by $\Delta x=a / 4$ to be exactly in the middle between apertures of pattern $a$. The lower surface of the wafer has some deep 
lines that are the result of manual polishing and which slightly reduce the quality of the mask layer on that side. Fortunately, the effect of such lines was found to be insignificant in subsequent processing, although it may introduce optical scattering.
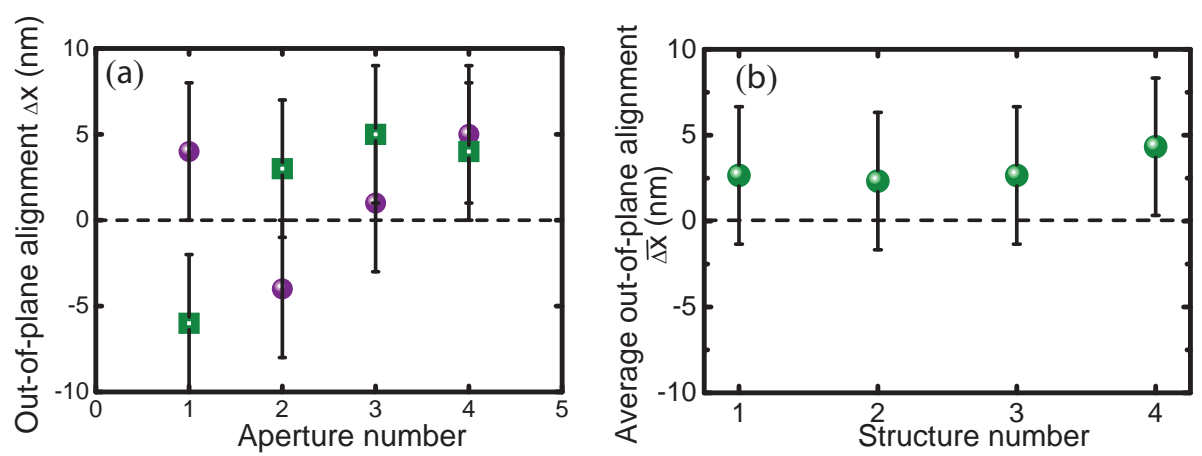

Figure 2.5: The out-of-plane alignment defined as a deviation $\Delta x$ of the position of an aperture in pattern $a$ relative to an aperture on the oblique plane in pattern $b$. The data were taken on the pattern shown in Fig. 9. (a) Circles show measurements taken for four pairs of apertures close to the wafer edge; apertures in pattern $a$ are located at $y=1.42 \mu \mathrm{m}$ and in pattern $b$ at $y=-1 \mu \mathrm{m}$. Squares show measurements for four pairs of apertures further from the edge; in pattern $a$ at $y=1.42 \mu \mathrm{m}$ and in pattern $b$ at $y=-5.42 \mu \mathrm{m}$. (b) Out-of-plane alignment averaged over four pairs of apertures $\overline{\Delta x}$ within one structure is shown for four different structures. In both figures error bars represent the resolution of the SEM.

Since patterns $a$ and $b$ are located on oblique planes we characterize the alignment between patterns $a$ and $b$ that we refer to as the out-of-plane alignment. We foresee two sources of possible misalignment: first, the sample may be placed under an angle different from $45^{\circ}$ during the mask projection step (Figure 2.2(b)), second, the mask pattern can be rotated with respect to the edge of the sample. In the first case the sample tilt is precisely set by a positioning stage and controlled with SEM image, therefore we expect the tilt alignment to be always better than a few degrees. For a misalignment by $2^{\circ}$ the dimensions of a projected pattern result in a structural strain of only $3 \%$. In the second case if the projected pattern is rotated with respect to the wafer edge by an angle $\delta$, the apertures located at a distance $y$ from the edge will be displaced by $\Delta x=y \cdot \sin \delta$ from their designed positions. In practice we expect the angular misalignment to be less than $\delta=2^{\circ}$, which is sufficiently small to realise bonafide 3D nanostructures.

To characterize the out-of-plane alignment we digitally take the grey value cross-sections from the SEM images through a row of apertures. First we select an aperture in pattern $a$ and take a grey value cross section from the SEM image to define its central position. Then we select an aperture in pattern $b$, take a grey value cross-section to define its position and compare the relative position 
$\Delta x$ of these two apertures. In this way we determine a mutual alignment of the apertures located on inclined planes. In Figure 2.5(a) we have collected two sets of data for the structure shown in Figure 2.8. In the $1^{\text {st }}$ set indicated as circles we plot the out-of-plane alignment between pairs of apertures where one aperture in pattern $a$ is taken in the row at a distance $y=1.4 \mu \mathrm{m}$ from the edge and the second aperture in pattern $b$ is taken in the row at a distance $y=-1 \mu \mathrm{m}$ from the edge. From the data we conclude that the deviation of apertures positions from the design is within the error bar of SEM accuracy. To verify the alignment over further distances from the edge, in the $2^{\text {nd }}$ set of alignment data, shown as squares in Figure 2.5(a), we take the second aperture in pattern $b$ in a row located at $y=-5.42 \mu \mathrm{m}$ and compare their positions to the same apertures in pattern $a$ as before. We see that the out-of-plane alignment stays within the resolution of SEM for both sets of data, independent of the distance from the edge, thus providing an upper boundary of $0.05^{\circ}$ for rotational misalignment discussed earlier. We find here the deviation from the designed structure (Figure 2.3 ) to be at most $5 \mathrm{~nm}$. In Figure 2.5(b) we plot the out-of-plane alignment data $\overline{\Delta x}$ that are averaged over four pairs of apertures within one structure for four different structures (as illustrated, e.g., in Fig. 4(a)). It is seen that the average over a mask varies between $\overline{\Delta x}=2.3 \mathrm{~nm}$ and $\overline{\Delta x}=4.3 \mathrm{~nm}$ with a mean of $3.0 \mathrm{~nm}$. The error bars in both Figures 2.5 (a) and 2.5(b) indicate the typical accuracy of $\pm 4 \mathrm{~nm}$ of the scanning electron microscope. Thus, we conclude that the outof-plane alignment data are mostly determined by the SEM error and are better than $3.0 \mathrm{~nm}$. Therefore, the out-of-plane alignment for individual apertures is consistent with zero deviation, in agreement with the starting point of our 3D mask method that the two oblique pattern have built-in mutual alignment.

Figure 2.4c shows a zoom-in to the front surface of a wafer. We see that the elliptical apertures in the pattern design (see Figure 2.3) have been correctly projected to become circular apertures with the diameter of $273 \mathrm{~nm}$ on the sample surface. From Figures 2.4 and 2.5 we conclude that we have successfully fabricated a desired 3D mask structure on two inclined planes. We emphasize that the alignment between the patterns on two inclined planes is ensured at the design stage since both patterns are written in one projection and is within the resolution of SEM.

After etching of deep pores in silicon in two perpendicular directions, we have sacrificed one crystal in order to view the internal structure of the sample by milling it with a focused ion beam. Figure 2.6(b) shows a schematic representation of the cut and a surface plane that is open for viewing. The cut was made under $45^{\circ}$ angle to both planes. Since the pore depth is finite, starting at a certain depth in the structure the perpendicular pores will not overlap inside the crystal. Pores that are far from the edge of a wafer are not deep enough in order to reach the corresponding perpendicular pore, see green line in Figure 2.6(c). Therefore we expect to see a region inside the crystal closer to the edge where pores overlap and form a 3D structure. Further from the edge we expect a region where the structure will be two dimensional. The SEM image of a crystal cross section is shown in Figure 2.6(a). We see that using a single step etch mask deep pores were successfully etched in silicon. Pores etched in both perpendicular 
a)

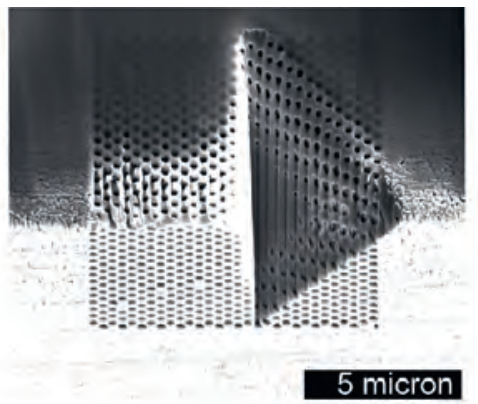

b)

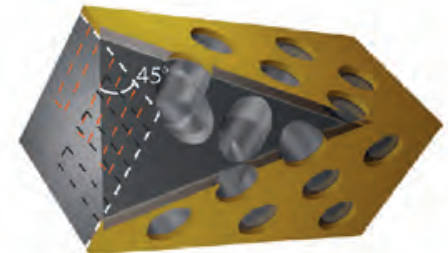

c)

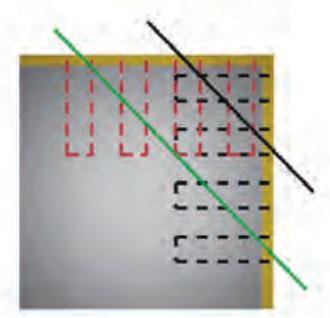

Figure 2.6: (a) The fabricated 3D diamond-like photonic crystal that was opened up by focused ion beam milling. Scale bar is shown in the picture. (b) Schematic representation of how a cross-section is milled on a fabricated sample. The cut is made at $45^{\circ}$ to the side of silicon bar. Dashed lines indicate the pore geometry. (c) Schematic cross-section that illustrates how limited pore depth appears on the milled structure. The black line shows where pores overlap with each other and the green line shows the depth beyond which the arrays of pores are two dimensional. (colour online)

directions form a 3D diamond-like structure in the bulk. Far from the surface of the Si wafer the pores are not overlapping. In the present case, the depth of the pores is determined to be $4 \mu \mathrm{m}$ deep with an aspect ratio (depth to width ratio) of 14 . The size of the fabricated photonic crystal is limited by the depth of the pores in the silicon [13]. We conclude from the cross-section that single step etch mask with ensured alignment allows us to fabricate a 3D monolithic nanostructure in bulk silicon.

\subsubsection{Structures feasible for fabrication}

There is a large variety of structures that can be fabricated using the presented technique. In general mask patterns projected in one step on inclined planes have an arbitrary structure and still be in perfect alignment with respect to each other. We consider 3D Bravais lattices that can be created with the described fabrication process. Using different pattern designs, it is possible to achieve structures with five Bravais lattice systems: cubic, monoclinic, orthorhombic, tetragonal and hexagonal. In Figure 2.7(a) on a left side is shown the simple cubic lattice. On the right side there is a pattern design for the fabrication 
of a simple cubic structure, where all lattice parameters are equal $(a=b=c)$. Taking into account that the pattern is projected on a $\theta=45^{\circ}$ inclined planes, in the design $a^{\prime}=a / \sin 45^{\circ}, c^{\prime}=c / \sin 45^{\circ}$ and $b^{\prime}=b$. This design consist of two rectangular arrays of apertures aligned with no shift with respect to each other. For completeness we note that this design for a cubic structure is not unique, as many different designs are feasible. In particular, the mask design presented in Figure 2.3 confirms that there are multiple possible designs for cubic structure, notable depending on the type of cubic symmetry (simple versus face centred). Figure 2.7(b) shows a simple tetragonal structure on the left side and the design pattern for realisation of such structure on two perpendicular planes on the right side. In case of tetragonal structure $a=b \neq c$, therefore in the design pattern $c^{\prime} \neq a^{\prime}$. That means that the complete mask pattern for a tetragonal structure consist of two different rectangular arrays of apertures, one with lattice parameters $a^{\prime}$ and $b^{\prime}$ and other one with parameters $c^{\prime}$ and $b^{\prime}$. Figure 2.7(c) shows the orthorhombic structure on the left and the corresponding design pattern on the right. For an orthorhombic structure $a \neq b \neq c$, which means that in the design pattern all lattice parameters are different. Hence the complete design consist of two different rectangular arrays of apertures with parameters $a^{\prime}, b^{\prime}$ and $c^{\prime}, b^{\prime}$. In Figure $2.7(\mathrm{~d})$ on the left side the monoclinic structure is shown, where $a=b=c$ and the angle between two lattice vectors is $\alpha \neq 90^{\circ}$. In the pattern design this means that the rows of apertures are shifted with respect to each other so that the angle between vectors $a^{\prime}, b^{\prime}$ and $c^{\prime}, b^{\prime}$ is $\alpha^{\prime}=\alpha / \sin 45^{\circ}$. In Figure 2.7(e) the hexagonal structure is shown on the left side and the pattern design on the right side. The structure consist of two hexagons shifted with respect to each other. Lattice parameters are $a=b \neq c$ and lattice angle $\alpha=120^{\circ}$. The pattern design consist of two completely different patterns. Pattern $a$ is similar to the well known hexagonal graphene-like pattern [18], whereas pattern $b$ has a rectangular array of apertures. Due to projection on the inclined planes the lattice parameters in the design are $a^{\prime}=a / \sin 45^{\circ}, c^{\prime}=c / \sin 45^{\circ}$ and $b^{\prime}=$ $b / \cos \alpha^{\prime}$, where $\alpha^{\prime}=\alpha / \sin 45^{\circ}$. The hexagonal mask has been realized in $\mathrm{Cr}$ on a Si wafer by means of a fabrication procedure described earlier in this article and is shown in Figure 2.8. The red dashed line shows the $90^{\circ}$ edge of a wafer. The top side on the picture is the (0001) crystal surface of hexagonal structure and the bottom side is a (1010) crystal plane. The 3D mask for this hexagonal structure shows clearly the flexibility of fabrication method to realise structures with independent patterns on inclined surfaces with an unprecedented out-ofplane alignment better than $5 \mathrm{~nm}$. All described 3D structures, except cubic, are predicted to reveal sub-Bragg diffraction [19] which makes them interesting subject for optical study, moreover, simple cubic and hexagonal structures have been predicted to reveal 3D band gaps [20, 21].

Besides different periodic structures it is possible to fabricate masks that yield non-periodic three-dimensional structures structures or periodic structures with controlled defects. An interesting example is the fabrication of a 3D photonic band gap crystal with a cavity inside. The geometry of cavity described in Reference [22] can be straightforward realised with the presented fabrication method by making two apertures for crossing pores on oblique planes smaller. Moreover, 

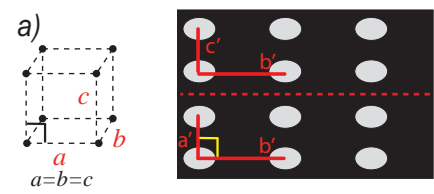

b)

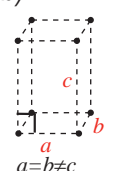

c)

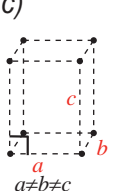

Pattern a

Pattern $b$
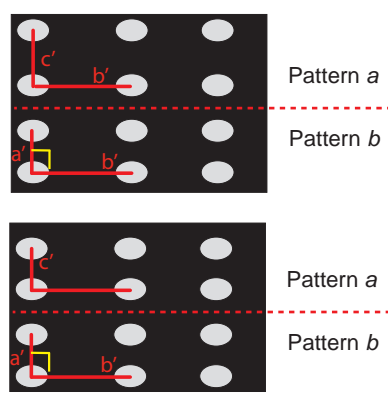

d)

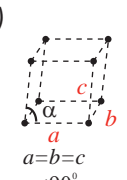

e)
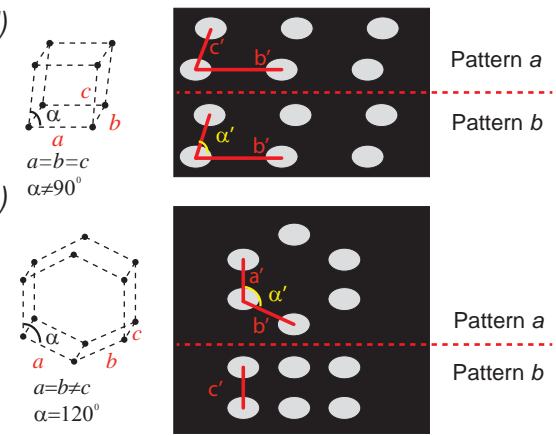

Figure 2.7: Pattern designs for different lattice systems fabrication. The 3D scheme of the structure is shown on the left and corresponding pattern for etch mask is on the right. (a) Cubic (b) Tetragonal (c) Orthorhombic (d) Monoclinic (e) Hexagonal

it is possible to realize an array of such cavities in a 3D photonic crystal. In Figure 2.9 the mask pattern for an array of $2 \times 2 \times 4$ cavities in 3D photonic crystal is shown. The resulting $3 \mathrm{D}$ array of band-gap cavities would represent the photonic version of the Anderson tight-binding model [23] that may reveal intricate nanophotonic phase transitions for light. Another example is the fabrication of three-dimensional disordered or aperiodic structures. In this case patterns $a$ and $b$ can be arrays of randomly distributed apertures or incommensurable lattices [25].

Since patterns $a$ and $b$ are independent from each other, it is also possible to fabricate functionally different components on inclined planes. Figure 1 in Reference [24] shows an illustration of a suggested chip consisting of two integrated circuits on adjacent planes that are interconnected. Using the presented fabrication method it is possible to make interconnection between different integrated circuits with ensured alignment. In addition, it is possible to project optically functional device on one surface and electronic components on the other surface. Such architecture greatly increases density of components on chip, makes interconnections between them easier and gives a possibility to spatially separate optics and electronics on chip.

The limitation for a number of possible structures comes from the subsequent silicon etching step. So far we assume that etching is always done with pores direction normal to the surface of the sample. Nevertheless there are examples where etching under an angle has been demonstrated [26] which can further increase the number of feasible structures. 


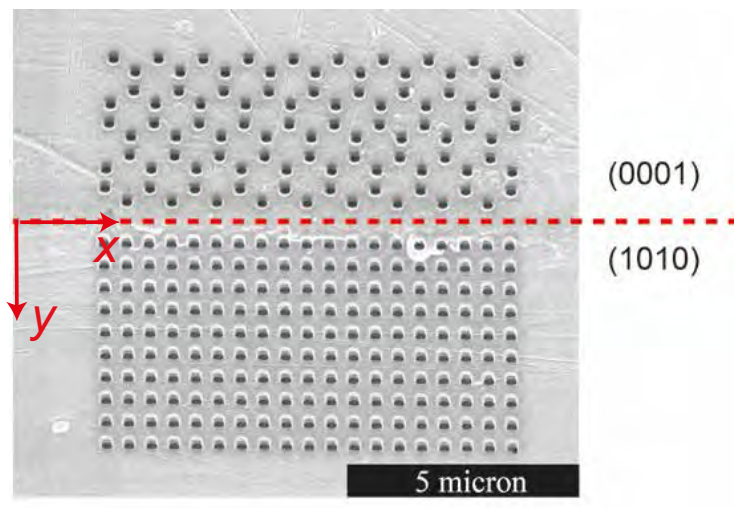

Figure 2.8: SEM image of a mask for hexagonal 3D crystal fabrication. The dashed line shows the $90^{\circ}$ edge of a wafer. Top side is a (0001) crystal plane of hexagonal structure and the bottom side is a (1010) crystal plane. The scale bar is shown on the picture.

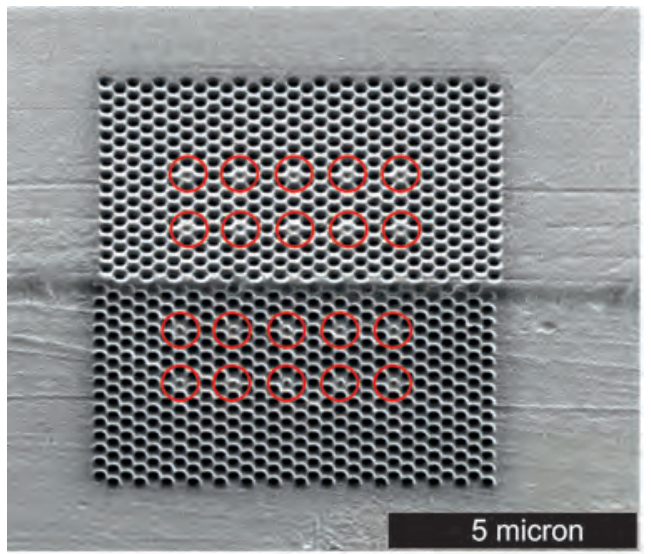

Figure 2.9: SEM image of a mask for an array of $2 \times 2 \times 4$ cavities in 3D photonic band gap crystal. Smaller pores that form cavities inside a structure are marked with red circles. (colour online)

\subsection{Conclusions and outlook}

In summary, a novel method has been proposed to fabricate a three-dimensional etch mask in one step with built-in alignment. The out-of-plane alignment between structures on to oblique adjacent planes has been characterized by means of deviation from the designed structure. The out-of-plane alignment has been found to be better than $3.0 \mathrm{~nm}$. A three-dimensional band gap photonic crys- 
tal with an inverse woodpile structure has been realized using a mask that we propose. The masks designs for 3D nanostructures with five different Bravais lattices, namely cubic, tetragonal, orthorhombic, monoclinic, and hexagonal has been shown. The mask for 3D hexagonal structure and for a 3D array of cavities had been realised on a $\mathrm{Si}$ wafer.

The presented method allows fabrication of 3D nanostructures that enable exciting prospects in nanophotonics including the pursuit of cavity resonances in 3D photonic band gap crystals [22] and 3D Anderson localization of light in photonic crystals $[23,27]$. The next step for further development of the single-step etch mask technique is to broaden it to different types of lithography discussed earlier. DUV lithography is particularly interesting since it is widely used in CMOS industry and therefore opens new possibilities notably for $3 \mathrm{D}$ photonic integrated circuits $[28,29]$. Also, patterning of inclined planes with accurate alignment allows one integrate on-chip different functional devices: for example an optical device on one surface and an electronic device on adjacent surface. We therefore foresee novel applications of our method in opto-electronics, integrated photonics and sensing. 



\section{Bibliography}

[1] Woldering L A, Mosk A P, Tjerkstra R W and Vos W L 2009 The influence of fabrication deviations on the photonic band gap of three-dimensional inverse woodpile nanostructures J. Appl. Phys. 10509310829

[2] Maldovan M and Thomas E L 2004 Diamond-structured photonic crystals Nature Mater. 3 593-600 29

[3] Ho K M, Chan C T, Soukoulis C M, Biswas R and Sigalas M 1994 Photonic band gaps in three dimensions: New layer-by-layer periodic structures Solid State Comm. 89 413-416 29

[4] Hillebrand R, Senz S, Hergert W and Gösele 2003 Macroporous-silicon-based three-dimensional photonic crystal with a large complete band gap J. Appl. Phys. 94275829

[5] L.A. Woldering, A.P. Mosk, R.W. Tjerkstra, and W.L. Vos, The influence of fabrication deviations on the photonic band gap of three-dimensional inverse woodpile nanostructures, J. Appl. Phys. 105, 093108: 1-10 (2009). 30

[6] van den Broek J M, Woldering L A, Tjerkstra R W, Segerink F B, Setija I D and Vos W L 2012 Inverse-woodpile photonic band gap crystals with a cubic diamond-like structure made from single-crystalline silicon Adv. Func. Mat. 22 25-31 30, 31

[7] Williams K R, Gupta K and Wasilik M 2003 Etch Rates for Micromachining ProcessingPart II J. Microelectromech. Syst. 12 761-778 30

[8] Imboden M and Bishop D 2014 Top-down nanomanufacturing Physics Today $6745-5030$

[9] Grivet P 1972 Electron optics (Oxford: Pergamon Press) p 50530

[10] Dwir B, Utke I, Kaufman D and Kapon E 2000 Electron-beam lithography of V-groove quantum wire device Microelectron. Eng. 53 295-298 30

[11] Smith C L C, Desiatov B, Giykmann I, Fernandez-Cuesta I, Levy U and Kristenses A 2012 Plasmonic V-groove waveguides with Bragg grating filters via nanoimprint lithography Opt. Express 20 5696-5706 30

[12] Banqiu W, Kumar A and Pamarthy S 2010 High aspect ratio silicon etch: A review J. Appl. Phys. 10805110131

[13] Woldering L A, Tjerkstra R W, Jansen H V, Setija I D and Vos W L 2008 Periodic arrays of deep nanopores made in silicon with reactive ion etching and deep UV lithography Nanotechnology 19145304:1-11 31, 35

[14] Schilling J, Müller F, Matthias S, Wehrspohn R B, Gösele U and Busch K 2001 Three-dimensional photonic crystals based on macroporous silicon with modulated pore diameter Appl. Phys. Lett. 78118031

[15] Föll H, Christophersen M, Carstensen J and Hasse G 2002 Formation and 
application of porous silicon Mat. Sci. Eng. R 39 93-141 31

[16] Schilling J and Scherer A 2005 3D photonic crystals based on macroporous silicon: Towards a large complete photonic bandgap Photonics Nanostruct. Fundam. Appl. 3 90-95 31

[17] Huisman S R, Nair R V, Woldering L A, Leistikow M D, Mosk A P and Vos W L 2011 Signature of a three-dimensional band gap observed on silicon inverse woodpile photonic crystals Phys. Rev. B 83 205313: 1-7

[18] Geim A K and Novoselov K S 2007 The rise of graphene Nature Mater. 6 183-191 36

[19] Huisman S R, Nair R V, Hartsuiker A, Woldering L A, Mosk A P and Vos W L 2012 Observation of sub-Bragg diffraction of waves in crystals Phys. Rev. Lett. 10808390136

[20] Cassagne D, Jouanin C and Bertho D 1996 Hexagonal photonic-band-gap structures Phys. Rev. B $\mathbf{5 3} 36$

[21] Sözüer S and Haus J W 1993 Photonic bands: simple-cubic lattice J. Opt. Soc. Am. B 2 296-302 36

[22] Woldering L A, Mosk A P and Vos W L 2014 Design of a 3D photonic band gap cavity in a diamond-like inverse woodpile photonic crystal Phys. Rev. B 9011514036,39

[23] Anderson P W 1958 Absence of Diffusion in Certain Random Lattices Phys. Rev 1091492 37, 39

[24] Tjerkstra R W, Woldering L A, van den Broek J M, Roozeboom F, Setija I D and Vos W L 2011 A method to pattern masks in two inclined planes for three-dimensional nano- and microfabrication J. Vac. Sci. Technol. B 29 061604: 1-8 30, 37

[25] Ghulinyan M and Pavesi L (eds) 2015 Ledermann A, Renner $M$ and von Freymann $G$ ch 7 Three-dimensional photonic quasicrystals and deterministic aperiodic structures (Cambridge: Cambridge University Press) 37

[26] Suzuki K, Kitano K, Ishizaki K and Noda S 2014 Three-dimensional photonic crystals created by single-step multi-directional plasma etching Opt. Expr. 22 17100-17106 37

[27] John S 1987 Strong localization of photons in certain disordered dielectric superlattices Phys. Rev. Lett. 58 2486-2489 39

[28] Chutinan A, John S and Toader O 2003 Diffractionless Flow of Light in All-Optical Microchips Phys. Rev. Lett. 9012390139

[29] Ishizaki K, Koumura M, Suzuki K, Gondaira K and Noda S 2013 Realization of three-dimensional guiding of photons in photonic crystals Nature Photon. 7 133-137 39 


\section{CHAPTER 3}

\section{Deep UV lithography on the inclined sidewall of a wafer}

In this chapter we present an original method to use conventional deep-UV step and scan lithography in combination with a prestructured substrate to pattern monolithic 3D nanostructures in only one step, with ensured out-of-plane alignment [1]. For the proof-ofconcept demonstration of the method we start with a silicon wafer structured with V-shaped trenches. Following the photoresist spin coating on the sidewall of the V-trenches, a deep UV exposure is performed to pattern the resist on the inclined sidewall of the Si. Thus apertures developed on the sidewall are located at different depths with respect to the $\mathrm{Si}$ wafer surface and therefore form the basis of a 3D structure made by etching. Apertures with diameter between 180 and $500 \mathrm{~nm}$ were successfully exposed up to $1.8 \mu \mathrm{m}$ deep on a silicon sidewall.

\subsection{Introduction}

Current fabrication technology in semiconductor industry is steadily developing and expanding in the direction of three-dimensional (3D) architectures. A large progress towards 3D architectures was achieved in the area of non-volatile memories driven by the increase of memory capacity while going from $2 \mathrm{D}$ to $3 \mathrm{D}$ architecture [2]. Modern flash memories, such as BiCS ("bit cost scalable") and NAND are already 3D structures [3,4]. A large demand for 3D nanostructures also arises in microelectroechanical (MEMS) or nanoelectromechanical (NEMS) technology $[5,6]$ where the structures typically have a very complex 3D geometry and are produced in a multiple-step layer-by-layer fabrication processes (that are in themselves planar steps). Besides an increasing commercial interest to push technologies to three dimensions, many new fundamental questions arise when the materials are structured in a 3D way. An example of a phenomena occurring only in 3D nanostructures is the full photonic band gap meaning that for a range of frequencies light is forbidden to propagate in any direction and for any polarization leading to the ultimate control of spontaneous emission [7]. Current open questions are whether $3 \mathrm{D}$ photonic crystals with embedded defects are suitable media for the long awaited experimental observation of 3D Anderson localization of light [9] or even suitable building blocks for 3D photonic integrated circuits 
(so-called PICs) [8]. To answer those questions there needs to be a way to fabricate in a CMOS-compatible manner $3 \mathrm{D}$ photonic crystals in a large scale to prevent finite size effects and fulfill industrial demands and with good alignment for controlling the geometries.

Despite the rapidly growing attention to $3 \mathrm{D}$ architectures, the platform for their fabrication are at this time purely two dimensional (2D). The most common way for industrial fabrication of 3D structures is layer-by-layer fabrication [10]. In this approach each layer is fabricated separately as a two-dimensional structure and all layers are stacked to form the 3D structure. An obvious issue in this approach is overlay: the alignment of every layer to the previous one. Thus, for fabrication of $N$ layered structure one needs $N-1$ alignment steps. Every alignment step introduces errors in the structure and moreover slows down the fabrication speed and thus the overall throughput of the fabrication process. A second issue in the layer-by-layer approach is interconnections between layers [11]. Some components that need to be connected to each other may often be located in different layers and thus the interconnection between them is complicated. On the other hand, features that are located close to each other within one layer have the problem of electrical and optical cross talk. Solving these problems is possible if the entire structure is built in a non-planar way such as, for example, feasible in the fast-rising multiphoton lithography also known as direct laser writing (DLW) [12]. The main current disadvantage of DLW is that fabrication is currently processed with polymer materials that are not typically used in optical or electronics. Thus in order to fabricate optically or electronically active device one needs to employ additional processing steps such as multiple infiltrations and calcinations that usually reduce the quality of the resulting structure by introducing the impurities, structural cracks, incomplete filling, see e.g. References $[13,14]$.

In this Chapter we introduce a novel concept for 3D nanofabrication using conventional UV lithography on a non-planar substrate. We propose to start fabrication process of $3 \mathrm{D}$ structures immediately from a non-planar pre-structured Si wafer and describe a process flow for making an etch mask on the inclined sidewalls of the wafer. The etch mask on the inclined sidewalls allows to directly etch structures in different depths of the wafer to form a 3D architecture. The wafer is pre-structured to differ from the planar geometry as shown in Figure 3.1(1). For the proof-of-principle experiment we choose to start from $\mathrm{V}$-shaped trenches on a Si wafer. The dimensions of the trenches can be varied depending on the required applications.

The next step for lithographic process is covering of the substrate with back anti-reflection coating (BARC) and photoresist as shown in Figure 3.1(2). Since the aim is to perform lithography on a sidewall, it is important that the sidewall of the trenches is covered with the photoresist homogeneously. After the sidewall is covered with photoresist, features can be exposed at different depths of the sidewall. Being exposed at different depths features are thus located in different planes with respect to each. As indicated with the black dashed line in Figure 3.1(3), the center of the aperture on the left sidewall is located in between the apertures on the right sidewall to form a $3 \mathrm{D}$ structure. Notably even when 
(1)
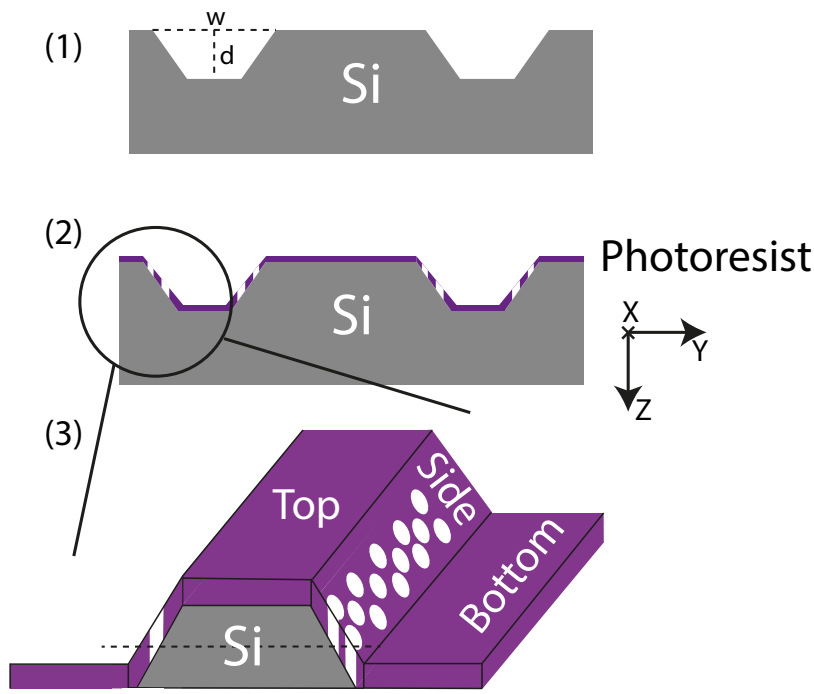

Figure 3.1: Concept of our lithography process for a 3D etch mask. The process starts with a pre-structured silicon wafer (1) with V-shaped trenches with width $w$ and depth $d$ that are made by standard cleanroom technologies. (2) The layer of photoresist is spin coated on the wafer and covers the sidewall of the trench to form a homogeneous layer. (3) Exposure of the features on the sidewall. Features are located on different $Z$-depths from the top of the trench and therefore are in different $(x, y)$ planes. As indicated with the black dashed line the center of the aperture on the left sidewall is intentionally in between the apertures on the right sidewall.

being located in different planes features are in perfect alignment with respect to each other since they are initially designed and projected as one image. Thus with just a single UV exposure on the pre-structured substrate, we realize a mask for etching in two non-parallel directions that allow to achieve a fully 3D structure.

\subsection{Experimental results}

\subsubsection{Process flow for V-trench fabrication}

To fabricate pre-structures silicon wafer with V-trenches as shown in Figure 3.1 we have designed a CMOS compatible process flow consisting of 7 steps that are schematically shown in Figure 3.2) and listed with detailed of process parameters in Table 3.1. In this process flow we use $\mathrm{KOH}$ anisotropic wet etching of Si to etch long trenches with well-defined sidewalls and inclination [15]. We start with a (100) silicon wafer covered with SiN layer that serves as a hardmask material for $\mathrm{KOH}$ etching (Figure 3.1, step 1). In the second step the photoresist layer is spin coated on top of the wafer. In order to etch trenches in a Si wafer using $\mathrm{KOH}$ 
(1)

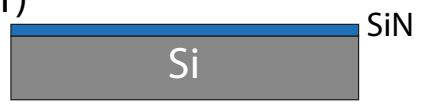

(2)
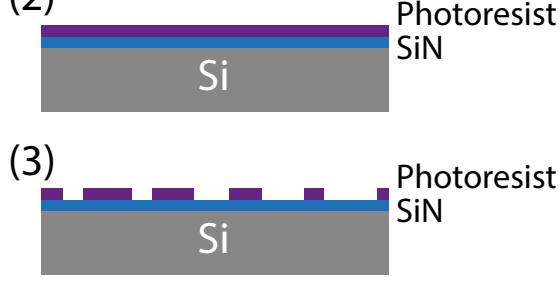

(4)

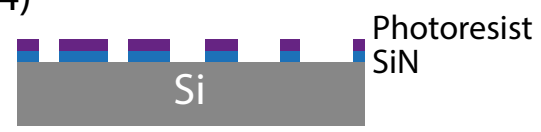

(5)

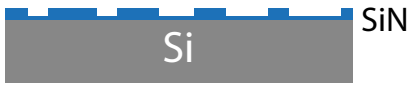

(6)

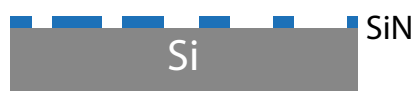

(7)

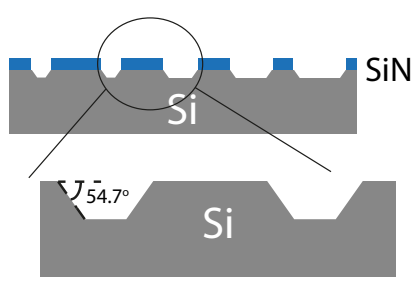

Figure 3.2: Schematic fabrication process for V-trenches on Si wafer. The detailed description of each fabrication step is given in Table 3.1.

wet etching [15] one needs to align the array of rectangles in the mask material parallel to the $<110>$ direction of the wafer. Thus special care has to be taken to carefully align the array of rectangles that are exposed in photoresist with respect to Si crystal planes. The alignment of the rectangles can be done either manually or by using standard procedure involving the fan alignment pattern described in reference $[16,17]$. After exposure and development of the pattern in photoresist it is transferred into SiN layer (step 3). The transfer is done by reactive ion etching (RIE) of SiN with parameters listed in Table 3.1. The etching time is chosen to be slightly shorter than needed to completely etch away SiN layer in order to prevent unwanted etching of Si underneath SiN. Therefore after stripping of photoresist with oxygen plasma (Step 4), a thin layer of SiN is still left in the lines that are supposed to be open. To completely remove both this layer as well as the native silicon oxide layer underneath a short wet etching in hot 50\% HF is applied (Step 6). The seventh step is anisotropic etching of the trenches in Si. KOH wet etching is chosen for anisotropic Si etching due to its known large ratio between etch rates of (110) and (111) Si crystal planes [20]. The wafer with SiN mask is placed in the $\mathrm{KOH}$ solution. Etching of the long lines parallel to $\langle 110\rangle$ direction in silicon wafer results in the grooves with 54.7 $\circ$ inclined sidewall [16] as shown in the Figure 3.2(7). The etching time in $\mathrm{KOH}$ defines the depth of the resulting trenches. After 6 minutes of etching the depth of the trenches will be $6 \mu \mathrm{m}$ [21] meaning that the trenches with a width of more than $w=8.76 \mu \mathrm{m}$ will have flat bottom (see Figure 3.2(7)). The optical microscope image of the resulting trenches on $\mathrm{Si}$ wafer is shown in Figure 3.3. The blue layer on the image is the remaining SiN mask layer. Bottom of the 


\begin{tabular}{|l|l|l|}
\hline Step & Description & Comments \\
\hline Start & $\begin{array}{l}(100) \text { Silicon wafer } \\
\text { deposition }\end{array}$ & Serves as an etch mask \\
\hline 2 & $\begin{array}{l}\text { Photoresist spin coating } \\
1.7 \mu m\end{array}$ & For pattern transfer to SiN \\
\hline 3 & $\begin{array}{l}\text { UV lithography exposure } \\
\text { and development }\end{array}$ & $\begin{array}{l}\text { Array of rectangles parallel to } \\
\text { the }<110>\text { crystal plane }\end{array}$ \\
\hline 4 & $\begin{array}{l}\text { RIE of SiN } \\
C H F_{3}: 5 \text { sccm } \\
O_{2}: 5 \text { sccm } \\
\text { Pressure: } 10 \text { mTorr } \\
\text { Time: } 1 \text { min } 50 \text { sec }\end{array}$ & $\begin{array}{l}\text { Pattern transfer from photoresist } \\
\text { to SiN }\end{array}$ \\
\hline 5 & Photoresist stripping & $\begin{array}{l}\text { To remove remaining SiN and } \\
\text { native oxide }\end{array}$ \\
\hline 6 & $\begin{array}{l}\text { Kot HF } 50 \%: 2 \text { min } 50 \text { sec } \\
\text { Temp.: } 75^{\circ} \mathrm{C}\end{array}$ & \begin{tabular}{l} 
Anisotropic etching of Si \\
\hline 7
\end{tabular}
\end{tabular}

Table 3.1: The process flow for $\mathrm{V}$ trenches fabrication on $\mathrm{Si}$ wafer

trenches are visible sharp since the microscope is focused on the bottom. The sidewall of the trenches is seen blurred since it is going in depth out of the focus range of the optical microscope.

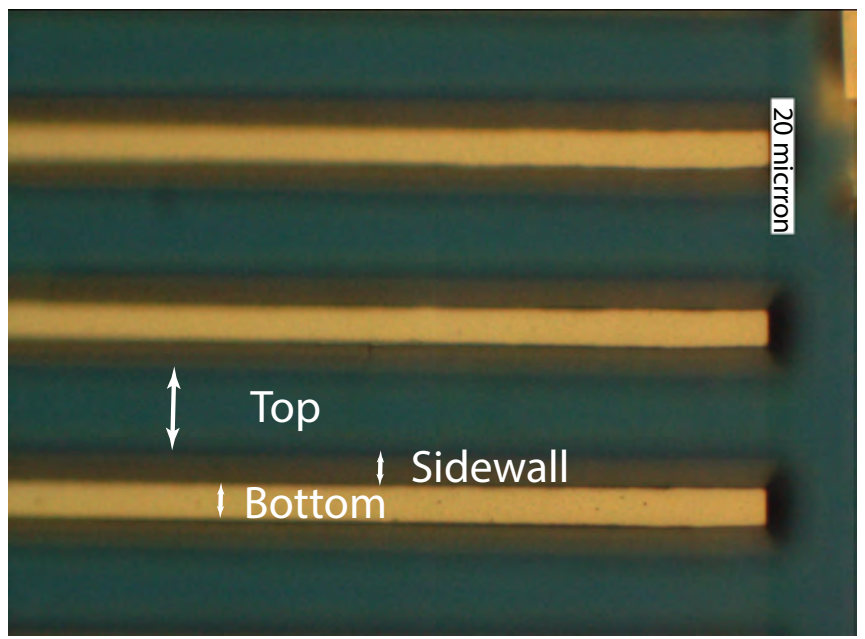

Figure 3.3: Optical microscope image of the trenches etched in silicon. Blue covering is the $\mathrm{SiN}$ etch mask. The width of the trenches is $w=15 \mu \mathrm{m}$. The $20 \mu \mathrm{m}$ scale bar is shown on the picture. 


\subsubsection{Spin coating of the sidewalls with photoresist}

After the trenches are formed the next step for UV lithography is the coating of the substrate consecutively with back anti-reflection coating (BARC) and photoresist. For the proof-of-principle experiment we require photoresist layer to have homogeneous withing $10 \mathrm{~nm}$ thickness along the area of exposure. In case of the flat substrate spin coating method is typically used to apply BARC and photoresist coatings. Alternative ways to coat the substrate are for example spray coating, dip coating or roll coating [18]. For the proof-of-principle experiment we choose spin coating as it is the most commonly used method. In a spin coating process the coating material is applied to the center of the rotating substrate. Fast spinning of the substrate ensures an even distribution of the coating along the substrate from the center to the edges. Although this method works well in case of a flat substrate it requires optimization in order to be applied for the non flat substrate such as Si with V-trenches. The experiments for optimization of the spin coating recipe were performed both in University of Twente Nanolab and in Nanolab of Technical University of Eindhoven (TU/e). In the University of Twente the coating experiments were performed on a Primus SB15 spin coater and in the TU/e the coating was applied using JDS-Uniphase Primer-oven system. The target of the experiments was to identify the thickness of the photoresist on the sidewall of the trenches on the Si wafer at different spin coating speeds and verify that homogeneous layer of photoresist is formed on the slope of a sidewall. For this experiment wafers were prepared according to the process flow described in section 3.2.1 consisting of the trenches with widths varying from 15 to $100 \mu \mathrm{m}$. After the spin coating wafers were cleaved and viewed with SEM in the cross section to identfy the thickness of the photoresist layer on the sidewalls. The pieces of the wafer chosen for the viewing were typically located in the central part of the wafer. The left sidewall and the right sidewall of the trench in a cross section are shown in the Figure 3.4(a). To verify the thickness along the slope of the sidewall multiple measurements were taken at different points. At 5 different positions on the slope of the sidewall photoresist thickness varied as little as $256 \mathrm{~nm}$ and $263 \mathrm{~nm}$ on both the left and the right sidewall. The important conclusion from this measurements is that the photoresist thickness is homogeneous on both sidewalls of the trench to within $7 \mathrm{~nm}$ along 2.6 microns of the slope which is sufficient for our proof-of-principle experiment.

Similar measurements were performed on the cross sections of the trenches with different widths and for wafers that were coated with two different spinning speeds: $4000 \mathrm{rpm}$ and $3000 \mathrm{rpm}$. The photoresist thickness measured at the half depth of the slope depending on the width of the V-trenches is plotted in the Figure 3.4(b). For both coating speeds there is a clear increase in the photoresist thickness with the increase of the trench width. This means that by designing trenches with different widths on the same wafer it is possible to achieve different resist thicknesses on the sidewalls depending on the requirements for the further processing. At the same time by varying the speed of the spin coating it is also possible to get different photoresist thicknesses on the sidewalls of the trenches. Thus there are two ways to achieve targeting resist thickness on the sidewall of 
the silicon wafer: first is to design trench width with respect to the given spin coating speed and second is to optimize the spinner speed to the given width of the trenches. This flexibility allows to vary the thickness of the targeted photoresist coating at multiple steps during fabrication process starting from the design of the pre-structured substrate and till the last moment before the spin coating. For our further exposure the targeted resist thickness was chosen to be $225 \mathrm{~nm}$. Given the targeted resist thickness for the DUV exposure we choose to have a substrate pre-structured with V-trenches with the range between $20 \mu \mathrm{m}$ and $40 \mu \mathrm{m}$ and spin coated with 3000 and $2500 \mathrm{rpm}$.
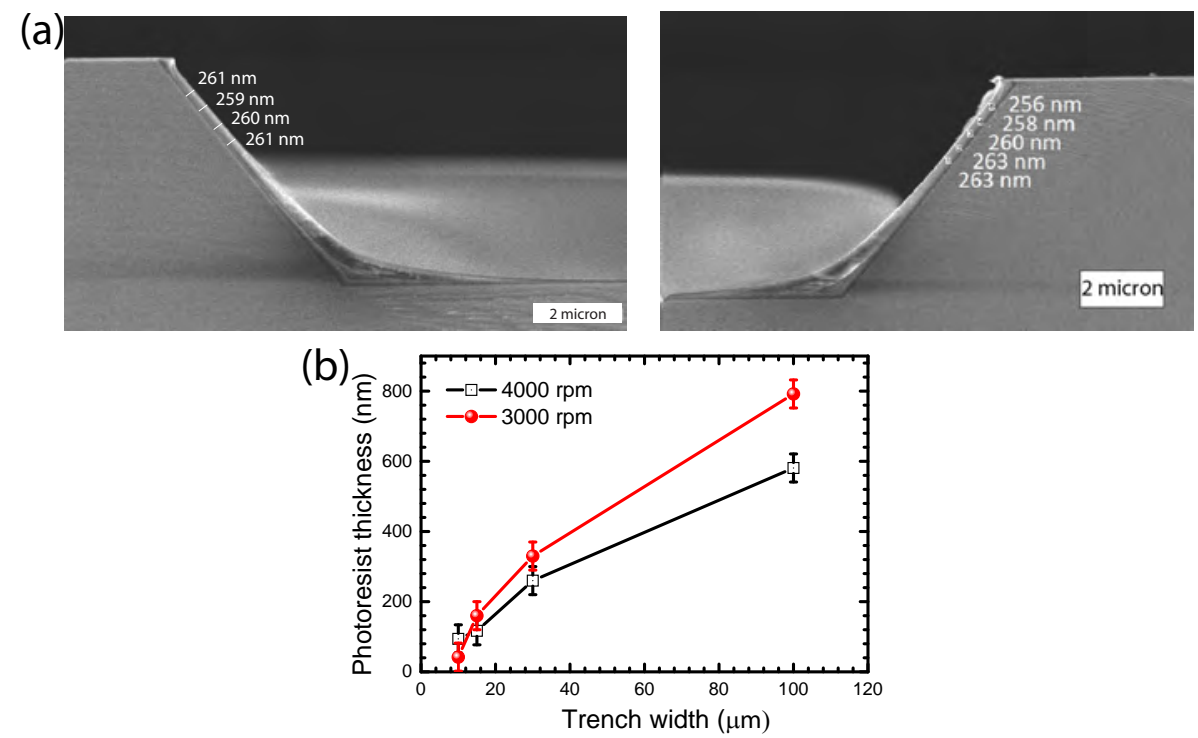

Figure 3.4: (a) SEM image of photoresist spin coated on the left and right sidewalls of the V-trench. The corresponding resist thickness measured at different points on the sidewall is indicated on the figure. The thickness of photoresist is similar on both sidewalls. The scale bar is shown on the image. (b) Thickness of the photoresist on a sidewall depending on a width of the $\mathrm{V}$-trench. Red line shows the resist thickness spin coated at $3000 \mathrm{rpm}$, black line shows the resist spin coated at $4000 \mathrm{rpm}$. The error bar indicates the

\subsubsection{DUV exposure with step-and-scan lithography}

For the exposure experiment we prepared two sets of wafers with V-trenches. Each set of wafers had trenches with depth varying from 20 to $40 \mu \mathrm{m}$. One set of wafers was spin coated with $3000 \mathrm{rpm}$ spinning speed and the second set of wafers was coated with $2500 \mathrm{rpm}$ spinning speed. The process flow for the coatings of the wafer is shown in Table 3.2. Each wafer was first coated with BARC, then with photoresist and last with top coating. The exposures of the 
photoresist on the sidewalls of $\mathrm{Si}$ wafer were performed with the ASML PAS $5500 / 1100 \mathrm{~B}$ stepper located in the TU/e NanoLab ${ }^{1}$. The stepper is equipped with a $193 \mathrm{~nm}$ ArF laser and $0.75 \mathrm{NA}$ lens providing $90 \mathrm{~nm}$ nominal spatial resolution. The exposure dose during the experiments was set to $25 \mathrm{~mJ} / \mathrm{cm}^{2}$. The layout of each wafer is made in such a way that there is a flat area in the middle of the wafer without any $\mathrm{V}$-trenches that is used for leveling in the stepper. Since our target is to expose apertures in depth on the inclined sidewall of the silicon wafer we set the nominal focus of the stepper to be $500 \mathrm{~nm}$ below the flat wafer level.

\begin{tabular}{|l|l|l|l|}
\hline Step & Description & Parameters & Comments \\
\hline 1 & Silicon wafer & & $\begin{array}{l}\text { Pre-structured with } \\
\text { V-trenches with 15 } \\
\text { to } 40 \quad \mu \mathrm{m} \mathrm{width} \\
\text { Table 3.1) }\end{array}$ \\
\hline 2 & Prime & & HMDS \\
\hline 3 & Chill anti reflection & $21^{\circ} \mathrm{C}$ for $60 \mathrm{sec}$ & \\
\hline 4 & $\begin{array}{l}\text { Bottom } \\
\text { coating BARC 1c5d }\end{array}$ & $38 \pm 3 \mathrm{~nm}$ \\
\hline 5 & Bake & $200^{\circ} \mathrm{C}$ for $90 \mathrm{sec}$ & \\
\hline 6 & Chill & $21^{\circ} \mathrm{C}$ for $60 \mathrm{sec}$ & \\
\hline 7 & Photoresist TARFP6111 & & $225 \pm 5 \mathrm{~nm}$ \\
\hline 8 & Soft bake & $125^{\circ} \mathrm{C}$ for $90 \mathrm{sec}$ & \\
\hline 9 & Chill & $21^{\circ} \mathrm{C}$ for $30 \mathrm{sec}$ & \\
\hline 10 & Top coat TCX041 & & $90 \pm 3 \mathrm{~nm}$ \\
\hline 11 & Soft bake & $90^{\circ} \mathrm{C}$ for $60 \mathrm{sec}$ & \\
\hline 12 & Chill & $21^{\circ} \mathrm{C}$ for $30 \mathrm{sec}$ & \\
\hline 13 & Exposure & $25 \mathrm{~mJ} / \mathrm{cm}^{2}$ & \\
\hline 14 & Developing OPD5262 & & \\
\hline 15 & Rinsing & $160^{\circ} \mathrm{C}$ for $90 \mathrm{sec}$ & \\
\hline 16 & Hard bake & & \\
\hline
\end{tabular}

Table 3.2: The process flow for the coating and exposure of silicon wafers using ASML PAS 5500/1100B stepper.

For the exposure of the apertures on the sidewalls of the V-trenches a special reticle was designed and bought by us. Our target is to expose circular apertures on the sidewalls that are inclined with $54.7^{\circ}$ with respect to the flat surface of the wafer (see Figure 3.2(7)). In order to get circles on the inclined surface one needs to project ellipses from above in the same way as it is done in the single step etch mask described in in chapter 2 section 2.2. Thus the design of the reticle consist of large fields of ellipses that result in the circular apertures on the

\footnotetext{
${ }^{1}$ It is a pleasure to thank Robert van der Laar, Jeroen Bolk, Kevin Williams, Huub Ambrosius and Meint Smit from TU/e for their advice and assistance in photoresist spin coating and DUV exposures and Eddy van der Heijde, Jo Finders and Arie den Boef from ASML for their help with DUV exposures.
} 
slope of the sidewalls. The small extraction from the reticle is shown in Figure 3.5. Elliptical apertures to be exposed in a photoresist are shown in blue. The arrangement of the apertures is centered rectangular with ratio $c / a=\sqrt{2}$. The size of the apertures varies across the reticle but the ratio $c / a$ is kept constant. By

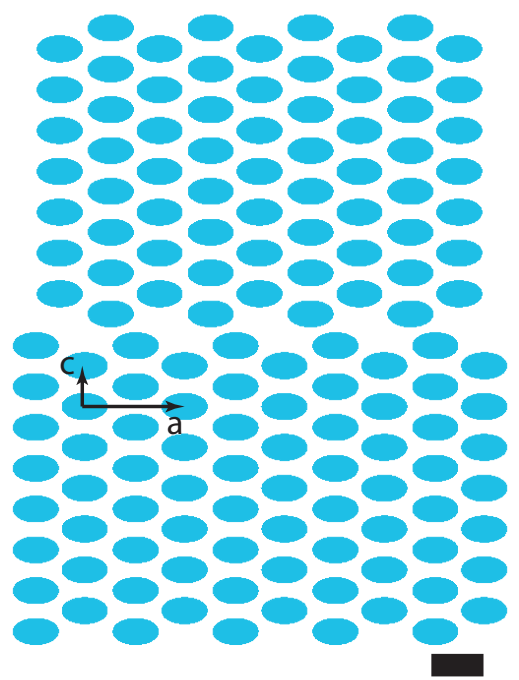

Figure 3.5: Detail of our new reticle design. The layout consists of large arrays of ellipses designed to appear as circles on the inclined slope. Apertures are arranges in a centered rectangular lattice with ratio $c / a=\sqrt{2}$. Scale bar of 1 micron is shown on the picture.

design, the apertures on the sidewall have a range of the diameters from $100 \mathrm{~nm}$ to $1000 \mathrm{~nm}$. The apertures are designed to form the centered rectangular lattice that is suitable for fabrication of for example 3D photonic band gap crystals (see Chapter 2, Figure 2.3). For the proof of principle experiment we aim to project apertures on anywhere on the sidewalls. To make it easier to find apertures in SEM after exposure we fill the entire wafer with apertures. Since there is are slight variations in the photoresist thickness along the sidewall we expect exposure to be successful at the point of the slope where resist thickness will be matching the dose, focus depth and aperture size.

\subsection{Results and discussion}

After the exposure all wafers were viewed with a critical dimensions SEM (CD SEM) Hitachi S-9920 to evaluate the exposure. The typical CD SEM image of the photoresist layer on the sidewalls of the wafers spin coated with $3000 \mathrm{rpm}$ after the exposure and development is shown in Figure 3.6. Wafers that were spin coated with $3000 \mathrm{rpm}$ speed did show neither individual apertures on the slopes of the sidewalls nor on the flat parts of the wafer. 
The flat top of the V-trench is on the left side of the image and the sidewall is going downhill from right to left. It can be seen that there are traces of apertures on the sidewall of the trench however the geometry of single apertures was lost likely due to the mismatch of the exposure dose and photoresist thickness .

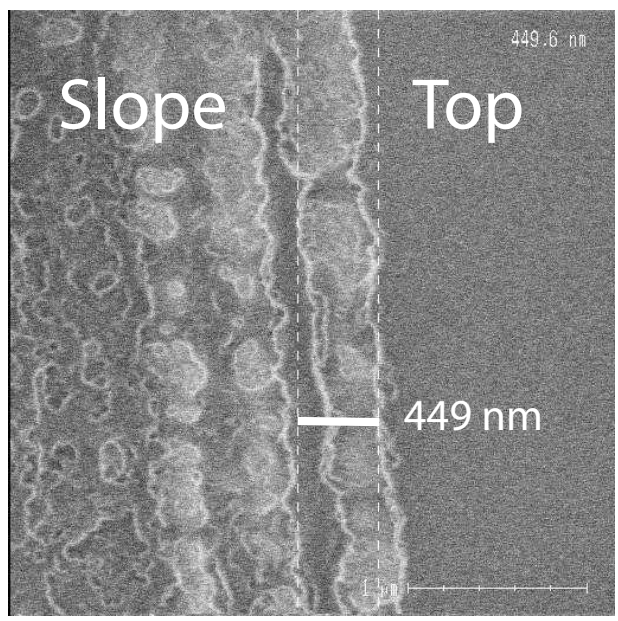

Figure 3.6: Typical exposure and development result on the wafers coated with 3000 rpm spinning speed. Traces of rows of apertures are visible on the slope of the trench. The geometry of individual apertures was lost likely due to the mismatch of the exposure dose and photoresist thickness.

However wafers that were spin coated with $2500 \mathrm{rpm}$ were exposed and developed successfully. Figure 3.7 shows the CD SEM images of the wafer that was coated with $2500 \mathrm{rpm}$ speed. In Figure 3.7(a) the top of the trench is on the right and the slope of the sidewall is going down from right to left. It is clearly seen that apertures of $509 \mathrm{~nm}$ size are fully open in the photoresist layer. Below the first row of apertures there is seen a signature of the second row of apertures thought apertures from the second row are seen less clear. We associate it with the fact that second row of apertures is located deeper on the slope thus further from the nominal focus of the exposure. Nevertheless for the proof of principle experiment even not fully developed apertures we count as a successful exposure. In Figure 3.7(b) another exposure and development on the slope of the sidewall is shown. The flat top of the wafer in on the left side and the sidewall slope is on the right side. The size of the fully developed aperture here is $412 \mathrm{~nm}$. There are two other rows of apertures apparent deeper down on the slope. Figure 3.7(c) shows exposure and development of apertures on another V-trench. In this case the right side of the trench is shown in order to prove that successful exposures are possible on both sides of the $\mathrm{V}$-trenches. Here the flat top part of the wafer is on the right side and the slope downhill is on the left side. The size of the open in photoresist aperture is $470 \mathrm{~nm}$. Figure 3.7(d) shows the smallest apertures that was found open on the wafers. Here the size of the apertures here is $180 \mathrm{~nm}$. Typically as feature size decreases their sensitivity to the focus errors increase 

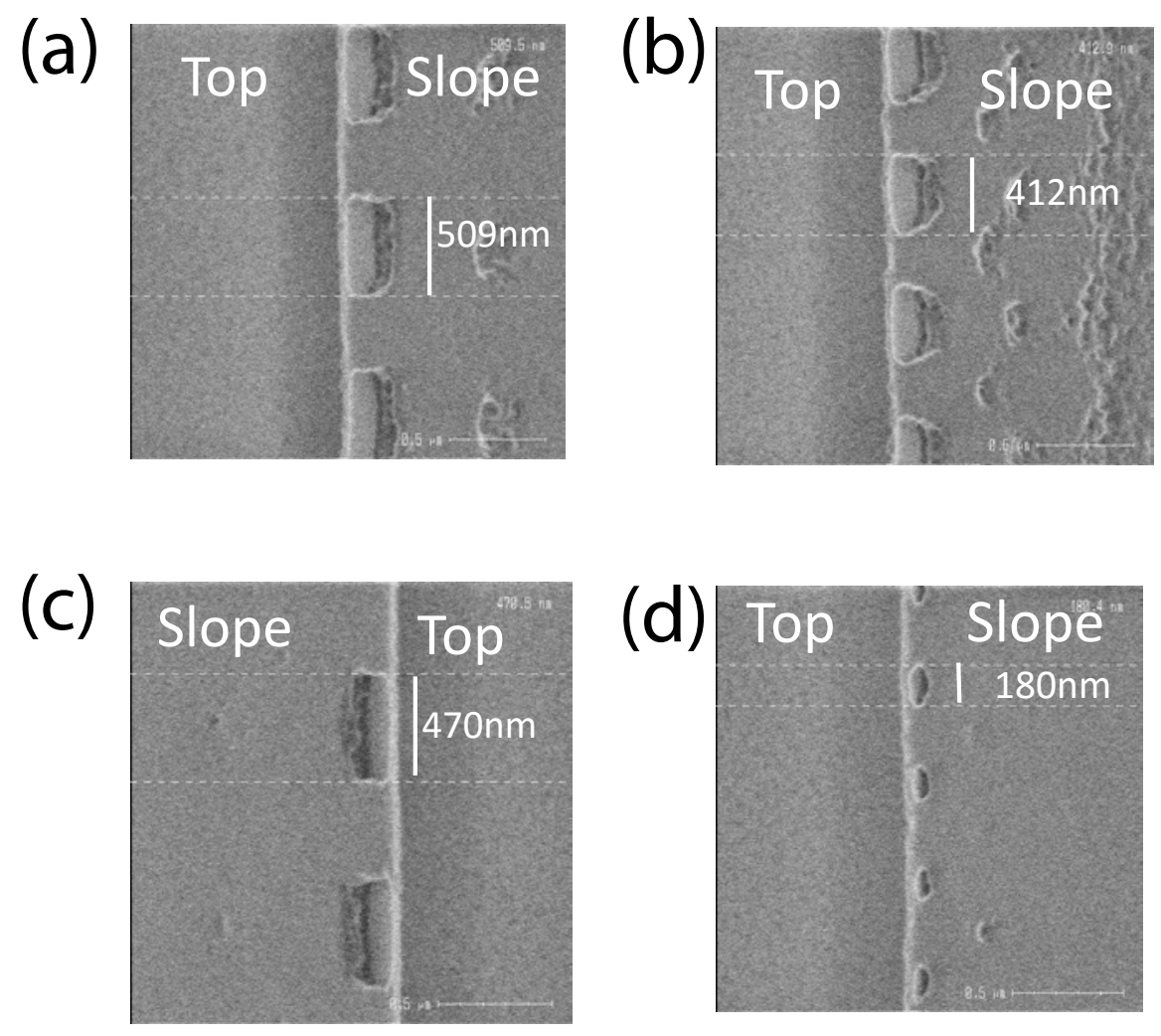

Figure 3.7: Apertures open in the photoresist on a sidewall of Si V-trench. (a) The flat top of the trench is on the left side and the sidewall is on the right side. A row of apertures is clearly visible to be developed on the sidewall close to the top surface. The size of each aperture is $509 \pm 5 \mathrm{~nm}$. One more row of apertures is less clearly seen deeper towards the bottom of the trench. (b) A row of apertures is open in the photoresist close to the top surface of the trench. The size of each aperture is around $412 \pm 5 \mathrm{~nm}$. Two more rows of apertures are visible deeper on the sidewall. (c) A row of apertures with $470 \pm 5 \mathrm{~nm}$ size is seen on the right side of the trench. We show here another side of the trench to indicate that successful exposure and development are possible on both sides of the trenches. (d) A row of apertures with the smallest size that was found on the wafer are seen on the sidewall. The size of each aperture is $180 \pm 5 \mathrm{~nm}$.

dramatically [23]. Thus it is reasonable that the smallest apertures are exposed best close to the nominal focal planes.

To evaluate the exposures in depth on the sidewall of V-trenches we measured the depth of the furthest open aperture of each size on the slope. The maximum depth at which we can still see the signatures of open apertures we call here maximum depth of exposure in order to not confuse with conventional definition 


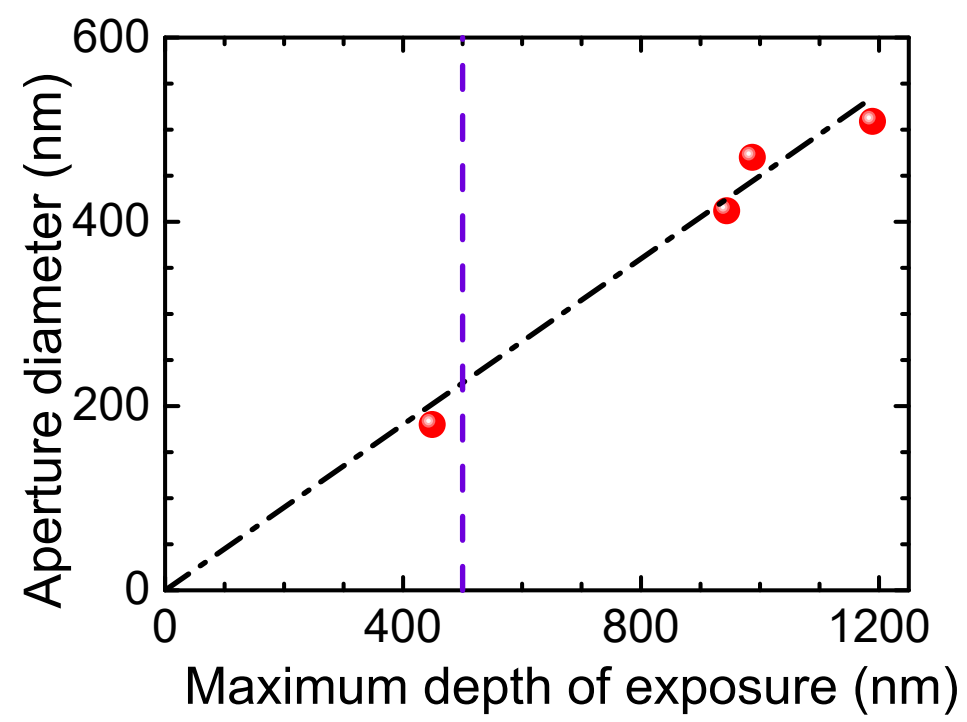

Figure 3.8: The maximum depth of exposure achieved on the sidewall of Si wafer depending on the aperture size. The purple dashed line indicates the nominal depth of the focus during the exposures. The dose during the exposure was $25 \mathrm{~mJ} / \mathrm{cm}^{2}$. Black dashed dotted line has a slope 0.45 , as a guide to the eye.

of depth of focus [23]. Figure 3.8 shows the maximum depth of exposure observed for apertures of each size. The largest apertures of around $500 \mathrm{~nm}$ were exposed up to $1.18 \mu \mathrm{m}$ down on the slope of the sidewall whereas the smalles apertures of $180 \mathrm{~nm}$ were successfully exposed at the maximum depth of $450 \mathrm{~nm}$. It is clearly seen that the maximum depth of exposure is larger for the large apertures which agrees with similar trend for the exposures on the flat wafers.

\subsection{Summary and outlook}

We have demonstrated first experiments for DUV lithography on the sidewall of Si trenches. We developed a process flow for pre-structuring Si wafers with Vtrenches for further DUV exposures. We showed that spin coating of photoresist on highly corrugated non planar substrate provides homogeneous coverage of the sidewalls with photoresist. The photoresist thickness on the sidewalls of $\mathrm{V}$-tenches was found to vary within $7 \mathrm{~nm}$ over the depth of 2.6 microns. We demonstrated exposure and development of apertures with diameters between 180 and $509 \mathrm{~nm}$ on located on the sidewalls of Si trenches. We found the maximum depth of focus for each aperture size. Apertures with largest size of $509 \mathrm{~nm}$ are located on the maximum depth of $1.18 \mu \mathrm{m}$. The smallest exposed and developed apertures are the apertures with $180 \mathrm{~nm}$ and the corresponding maximum depth of focus is found to be $449 \mathrm{~nm}$. In our experiments we observed only one row 
of apertures to be fully exposed and developed on the sidewall. While one row of apertures is not sufficient to fabricate a $3 \mathrm{D}$ photonic crystal, the required 4 to 5 rows (from Equation 1.6 we derive $L_{\text {Bragg }} \approx 3.2 d$, where $d$ is a lattice spacing) could be achieved by for example consecutive lithography imaging on consecutive depths. In order to improve fabrication process care needs to be taken in the design of the trenches since photoresist thickness on the sidewalls depends on the width of the trenched and should be optimized. A special reticle with apertures that will be aligned with respect to the trenches on located exactly on the slope will also help in achieving better exposure results. A process flow needs to be designed to transfer the pattern from photoresist to silicon. This can be done with deep reactive ion etching. This results open a number of new opportunities for 3D nanofabrication by means of conventional DUV lithography such as for example possibility to fabricate wafer scale 3D photonic band gap crystals with seemingly endless length along the trenches or hybrid functional nanostructures such as photonic integrated circuits (PIC) in close proximity to e.g. memory circuits or electronic data processing circuits. 



\section{Bibliography}

[1] D.A. Grishina, C.A.M. Harteveld, L.A. Woldering, and W.L. Vos, Method to make a single-step etch mask for 3D monolithic nanostructures, Nanotechnology 26, 505302 (2015)

[2] Y. Fujisaki Review of Emerging New Solid-State Non-Volatile Memories Jpn. J. Appl. Phys. 52, 040001:1-11 (2013) 43

[3] H. Tanaka, M. Kido, K. Yahashi, M. Oomura, R. Katsumata, M. Kito, Y. Fukuzumi, M. Sato, Y. Nagata, Y. Matsuoka, Y. Iwata, H. Aochi, and A. Nitayama Bit Cost Scalable Technology with Punch and Plug Process for Ultra High Density Flash Memory Proc. Int. Symp. VLSI Technology, 14 (2007) 43

[4] S. Whang, K. Lee, D. Shin, B. Kim, M. Kim, J. Bin, J. Han, S. Kim, B. Lee, Y. Jung, S. Cho, C. Shin, H. Yoo, S. Choi, K. Hong, S. Aritome, S. Park, and S. Hong Novel 3-Dimensional Dual Control-Gate with Surrounding FloatingGate (DC-SF) NAND Flash Cell for 1 Tb File Storage Application IEDM Tech. Dig. 10, 668-671 (2010) 43

[5] F. Niklaus, M. Lapisa, S.J. Bleiker, V. Dubois, N. Roxhed, A. C. Fischer, F. Forsberg, G. Stemme, D. Grogg, M. Despont Wafer-level heterogeneous 3D integration for MEMS and NEMS LTB-3D, 247-252 (2012) 43

[6] V.Ya. Prinz, V.A. Seleznev, A.V. Prinz, and A.V. Kopylov 3D heterostructures and systems for novel MEMS/NEMS J. Micromech. Microeng. 20 015021:1-11 (2010) 43

[7] E. Yablonovitch Inhibited spontaneous emission in solid-state physics and electronics Phys. Rev. Lett. 58, 2059-2062 (1987) 43

[8] K. Ishizaki, M. Koumura, K. Suzuki, K. Gondaria, and S. Noda Realization of three-dimensional guiding of photons in photonic crystals Nature Photon. 7133-137 (2013) 44

[9] S. John Strong localization of photons in certain disordered dielectric superlattices Phys. Rev. Lett. 58, 2486-2489 (1987) 43

[10] A. Todori-Sanial and C.S. Tan Physical design for $3 D$ integrated circuits CRC Press (2016) 44

[11] V. Kumar, H. Oh, X. Zhang, L. Zheng, M. S. Bakir, and A. Naeemi Impact of On-Chip Interconnect on the Performance of 3-D Integrated Circuits With Through Silicon Vias: Part I IEEE Trans. Electron Devices 63, 2503-2509 (2016) 44

[12] M. Deubel, G. von Freymann, M. Wegener, S. Pereira, K. Busch, and C.M. Soukoulis Direct laser writing of three-dimensional photonic-crystal templates for telecommunications Nat. Mater. 3 444-7 (2004) 44

[13] J. E. G. J. Wijnhoven, L. Bechger, and W. L. Vos Fabrication and charac- 
terization of large macroporous photonic crystals in titania Chem. Mater. 13 4486-4499 (2001) 44

[14] L. Bechger Synthesis and Fluorescence of Opal 83 Air-Sphere Photonic Crystals, PhD thesis (2003) 44

[15] M. Elwenspoek, H.V. Jansen Silicon micromachining (Cambridge Studies in Semiconductor Physics and Microelectronic Engineering) Cambridge University Press, 1999 45, 46

[16] T.D. James, G. Parish, K.J. Winchester, and C.A. Musca A crystallographical alignmnet method in silicon for deep, long microchannel fabrication J. Micromech. Microeng. 16, 2177-2182 (2006) 46

[17] W.-H. Chang and Y.-C. Huang A new pre-etching pattern to determine h110i crystallographic orientation on both (100) and (110) silicon wafers Microsys. Technol. 11, 117-128 (2005) 46

[18] S. Franssila Introduction to Microfabrication John Wiley \& Sons, Ltd (2004) 48

[19] D.L. Kendall On etching very narrow grooves in silicon Appl. Phys. Lett. 26, 195-198 (1975)

[20] K. Sato, M. Shikida, Y. Matsushima, T. Yamashiro, K. Asaumi, Y. Iriye, and M. Yamamoto Characterization of orientation-dependent etching properties of single-crystal silicon: effects of $\mathrm{KOH}$ concentration Sens. Actuators A 64, 8793 (1998) 46

[21] W.R. Runyan and K.E. Bean Semiconductor Integrated Circuit Precessing Technology Reading, MA: Addison-Wesley (1990) 46

[22] W. M. Moreau Semiconductor Lithography Principles, Practice, and Materials Plenum Press. New York (1988)

[23] C.A. Mack Fundamental Principles of Optical Lithography: The Science of Microfabrication John Wiley \& Sons, Ltd (2007) 53, 54 


\section{CHAPTER 4}

\section{Deep reactive ion etching of silicon}

We present advanced nanofabrication process realized in two steps on two inclined surfaces of a silicon wafer. Such process is required for the fabrication of $3 \mathrm{D}$ nanostructures in silicon. We discuss here $\mathrm{Cr}$ hard mask deposition and milling on two faces of silicon wafer as well as deep reactive ion etching (DRIE) process required for successful fabrication of 3D nanostructures. We fabricate 3D inverse woodpile photonic band gap crystals using our single step $\mathrm{Cr}$ etch mask discussed in details in the previous chapter and DRIE process described here. We also present nanopores with the highest reported aspect ratios etched by means of DRIE in silicon: the etched structures have a diameter of $510 \mathrm{~nm}$ and a depth of $\mathbf{2 1 . 5}$ microns resulting in a high aspect ratio of 42. The advances in the etching of deep pores in silicon offers exciting opportunities for both two-dimensional and three-dimensional silicon fabrication in the areas of photonic crystals, sensing, solar cells, highfrequency electronics and many others.

\subsection{Introduction}

Since silicon is the main material in modern CMOS industry, its processing attracts particular attention [1]. Growing attention for three-dimensional devices requires creative approaches in their fabrication. One of the challenges related to the increasing dimensionality of the devices is the fabrication of structures in silicon with high aspect ratio. One of the most used tools for high aspect ratio (HAR) structures fabrication in silicon is deep reactive ion etching (DRIE) [2]. In order to form a three-dimensional structure it is necessary to perform etching in multiple directions. This can be done by simultaneous multidirectional etching with the control over the ion sheath as shown in Reference [9]. Even though there are successfully fabricated photonic crystals using this approach, a strong limitation on the size of fabricated structure to 2 lattice periods may prevent the formation of the complete band gap. Moreover, there is a strong limitation on the geometry of fabricated structure since one and the same mask is necessarily used for etching in different directions. Another approach for multidirectional etching is shown in Reference [10] with the use of Faraday cage around the sample in the etching chamber. The cage provides the equipotential surface for the etching plasma ensuring that ions propagate normal to its surface. Such an approach 
requires a significant modification to the etching chamber and extensive simulations of the cage angle, mesh size and the sample placement. Thus for fabrication of our structure of inverse woodpile (see Chapter 1, Figure 1.2) consisting of two perpendicular array of pores we choose to perform etching in two steps with 90 deg rotation of the sample in between. The first successes in this respect were reported by van der Broek et. al. [11] and here we extend this approach to much deeper pores and new two-step etch mask.

\subsection{General description of deep reactive ion etching}

Deep reactive ion etching is a dry plasma type of etching [2]. While DRIE can be performed with many various types of etching gases, in this Chapter we choose $\mathrm{SF}_{6}$ gas as an etchant for $\mathrm{Si}$, and $\mathrm{O}_{2}$ for cleaning. The schematic of the etching chamber is shown in Figure 4.1. The pressure in the chamber is regulated by vacuum pumps and valves. The gas flow that is fed to the chamber is regulated with the entry valves. For stability and temperature control the helium backside cooling is provided. For high aspect ratio etching the chamber is typically featured with capacitive coupled plasma source (CCP) and inductively coupled plasma source (ICP) for separate control of ion energy and ion density respectively (Figure 4.1). The ICP plasma source generates plasma remotely and $\mathrm{CCP}$ generator is coupled to the lower electrode to induce the DC bias. Hence the ion density is controlled by ICP power and ion energy is controlled by CCP power supply. High ion densities increase the directionality of the etching but at the same time due to the ions collisions the angular distribution of ions leads to the unwanted sidewall erosion. The composition of the plasma in the chamber is very complex but generally in case of $S F_{6}$ etchant it contains $F \cdot$ radicals and $S F_{x}^{+}$ ions [3]. The radicals are not influenced by the electric field in the chamber and thus do not have a preferable direction of movement. The directionality of the etching is employed by the ions that are directed towards the lower electrode by the electric field (see Figure 4.1). If the applied electric field is high then physical sputtering of the material from the surface may occur. Micro-masking effects appearing due to the physical sputtering [4] may also contribute to the sidewall erosion. The etching process that is used in this Chapter for the fabrication of deep structures in silicon is the two-step Bosch process [8]. In this process the etching step and the protective deposition step are continuously alternated. During the deposition step a passivation gas is entered into the reaction chamber, in our case it is $C_{4} F_{8}$. From the $C_{4} F_{8}$ plasma a protective layer of polymer is deposited over the whole surfaced present in the chamber including sidewalls and and bottom of the etched structures. During the next etching step the protective layer is removed from the horizontal surfaces due to the vertically directed ions bombardment. Thus passivation layer protects the sidewalls and ensures high aspect ratio etching. Careful manipulation of both etching and deposition steps duration, gas flows and powers of the plasma sources is required to achieve deep structures without significant sidewalls erosion. The maximum depth of the structure that can be etched with the Bosch process depends on the opening in 


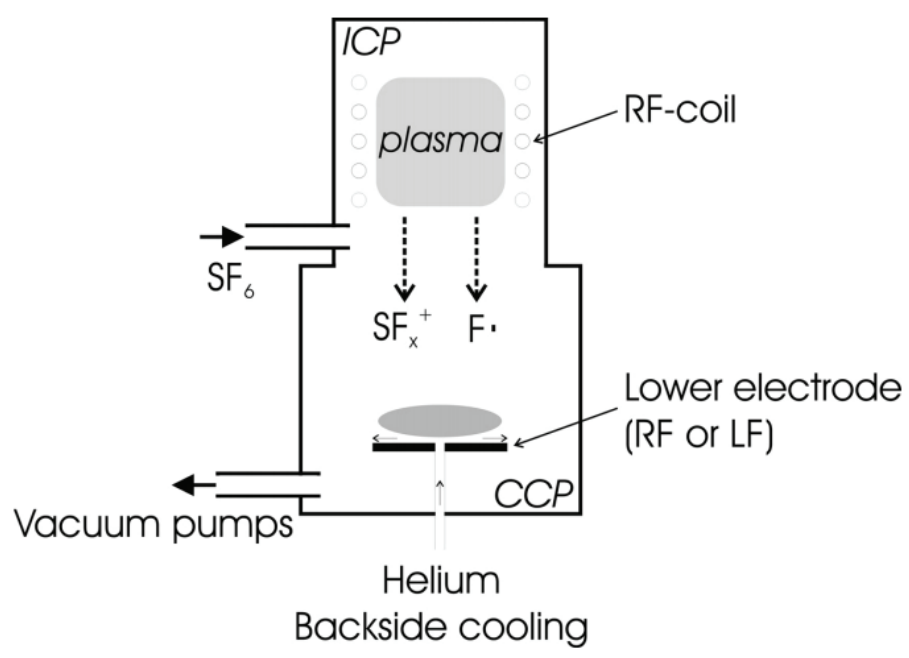

Figure 4.1: The schematic picture of a DRIE chamber with inductively coupled plasma source (ICP) and capacitively coupled plasma source (CCP). Vacuum pumps and entry valves regulate the pressure and the flow of etchant gas. The plasma consisted of ions and radicals is generated by the ICP plasma source and ions are accelerated towards the sample (gray ellipsoid) by CCP plasma source. Helium backside cooling provides temperature regularization of the substrate. The figure is adopted from the Reference [7].

the etch mask through which the etching is occurring. In the case of pores the maximum depth of the etched pore will depend on the pore diameter thus to characterize the etching process we will use aspect ratio rather than pore depths. The maximum aspect ratio that is possible to achieve with Bosch process is limited due to the changes in ions angular distribution in the depth of the etched structure and the difficulty for the etching gas to penetrate deeper in the pores.

\subsection{Experimental}

In this Chapter we study DRIE etching with Bosch process performed on two etching machines - Adixen Alcatel AMS100SE (from now on shortly referred to as Adixen) and SPTS Pegasus etching machine (referred to as SPTS). Due to the differences in two machines the optimized recipes for etching deep pores in Si will be listed separately for each machine. We use single crystalline silicon $<110>$ p-type wafer as a substrate to be etched. For fabrication of 3D photonic crystals small Si bars are etched from the substrate such that there are two perpendicular surfaces open for etching. Both perpendicular surfaces are covered with $50 \mathrm{~nm}$ layer of sputtered $\mathrm{Cr}$ that is used as a hard mask material. We define the apertures in the etch mask for 3D photonic crystal fabrication by means of focused ion beam lithography as described in the Chapter 2. For the 
test of etching parameters we use flat $\mathrm{Si}$ wafers with $\mathrm{Cr}$ etch mask patterned with centered rectangular array of apertures by means of DUV lithography. The pattern is chosen such that the wafer always cleaves through a set of pores and thus is referred to as an "easy cleave" pattern. The pattern design has $84.5 \mathrm{deg}$ angle between the axis of the primitive lattice. The details of the "easy cleave" pattern can be found in Reference [7]. For the test of parameters wafer with 2D pattern is cleaved into small pieces that are then places in the etching chamber on top of a dummy wafer. Si bars for etching of 3D photonic crystals are placed on the dummy wafer as well. Since DRIE etching is known to have the load effect [4] such that the etch rate depends on the amount of the surface that is being etching it is important to control the amount of surface that is etched. Thus we need to coat the silicon surface of a dummy wafer. To do so a dummy silicon wafer is always covered with a photoresist layer according to the recipe in the Table 4.1. For better temperature conductivity between the sample and dummy wafer a droplet of a vacuum oil is applied underneath the sample.

\begin{tabular}{|c|c|}
\hline Step & Comments \\
\hline Primer spin coating & HMDS \\
\hline Photoresist coating & Olin OIR $907-17$ \\
\hline Baking & $\mathrm{T}=120 \mathrm{C}$ for $3 \mathrm{~min}$ \\
\hline
\end{tabular}

Table 4.1: The process flow for the coverage of dummy wafer with the photoresist

\subsection{Ultra-high aspect ratio nanopores in $\mathrm{Si}$}

$2 \mathrm{D}$ arrays of deep pores are the building blocks for 3D inverse woodpile photonic crystal structures (see Chapter 1 Figure 1.2) that are discussed in this thesis. To maximize the size of the 3D structure it is necessary to make pores as deep as possible. Moreover for the good optical performance the sidewall erosion should be minimized to reduce undesirable light scattering in the structure. Thus care needs to be taken of this two parameters. Let us first consider the sidewall erosion present in the pores after the etching. Bosch process is known to have a systematic roughness arising from the cycling nature of the etching process. Such roughness is a sequence of rims and is shown closely on the top inset in Figure 4.2. This type of erosion is commonly referred to as a scallop in literature[5]. The scallop is typically more pronounced in the top part of the etched structure as can be seen from Figure 4.2 and this is because the top part of the etched structure is experiencing larger number of etching cycles. The top part scallop present in the Figure 4.2 is periodic with periodicity around $180 \mathrm{~nm}$ and thus we expect it to have small influence on the scattering of light of wavelengths within the range of our interest ( $\mathrm{f} \lambda$ from $800 \mathrm{~nm}$ to $2500 \mathrm{~nm}$ ). The lower inset in Figure 4.2 shows the image of the bottom of the etched pores taken with helium ion microscope (HIM) at ultra high resolution of less than $1 \mathrm{~nm}$. It reveals for the first time that scallop of the Bosch etching is also present at the very bottom of the etched structure and moreover shows the extreme smoothness of the walls of 


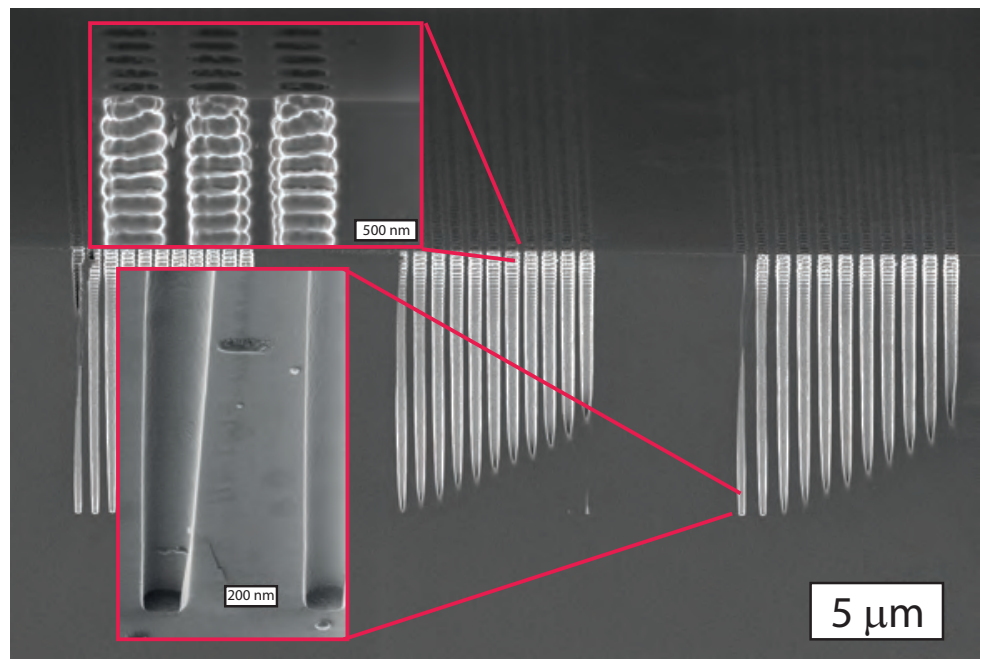

Figure 4.2: SEM image of the cross section of the 2D array of pores with $250 \mathrm{~nm}$ radius etched with Bosch process. 2 micron scale bar is shown on the picture.

the pores above the scalloping region and impressively flat bottom. From this images we conclude that no further specific optimization of the sidewall erosion is needed for our structures at the current stage.

The deepest pores we achieved were etched with the Bosch process on the SPTS etching machine. The process flow that was used for etching is listed in the Table 4.2. The process flow is based on the etching method reported in the work by L.A. Woldering et al. [6, 7]. The difference between the process flow we are using and the process flow suggested in the Reference [7] is the CCP power. The optimum CCP power differs due to the differences in matching the CCP

\begin{tabular}{|c|c|}
\hline Parameter & Value \\
\hline$S F_{6}$ gas flow & $62 \mathrm{sccm}$ \\
\hline$C_{4} H_{8}$ gas flow & $200 \mathrm{sccm}$ \\
\hline Etching step duration & 3 seconds \\
\hline Deposition step duration & 2 seconds \\
\hline CCP power & Varied from 30 to $70 \mathrm{~W}$ \\
\hline ICP power & $1500 \mathrm{~W}$ \\
\hline
\end{tabular}

Table 4.2: The process flow for DRIE of HARS in Si with the SPTS machine.

source in the SPTS etcher used in this section and Adixen etcher used in the work of Reference [7]. The CCP power of $150 \mathrm{~W}$ suggested in the Reference [7] resulted in a strong overetching of the structure such that it is not feasible to define pore depths or diameters after etching. Thus we tuned the CCP power down and performed series of experiments with CCP power in the range between 20 and $70 \mathrm{~W}$. As a figure of merit for the etching process we provide here the 
maximum aspect ratio measured from the cleaved pores. The maximum aspect ratio obtained in the etched structures at different CCP is shown in Figure 4.3. SPTS etcher has a possibility to ramp the CCP power during etching cycle. Thus when we plot a value of $35 \mathrm{~W}$, this implies that the power was set to be ramped up from 30 to $40 \mathrm{~W}$. It is seen from Figure 4.3 that the larger CCP power leads

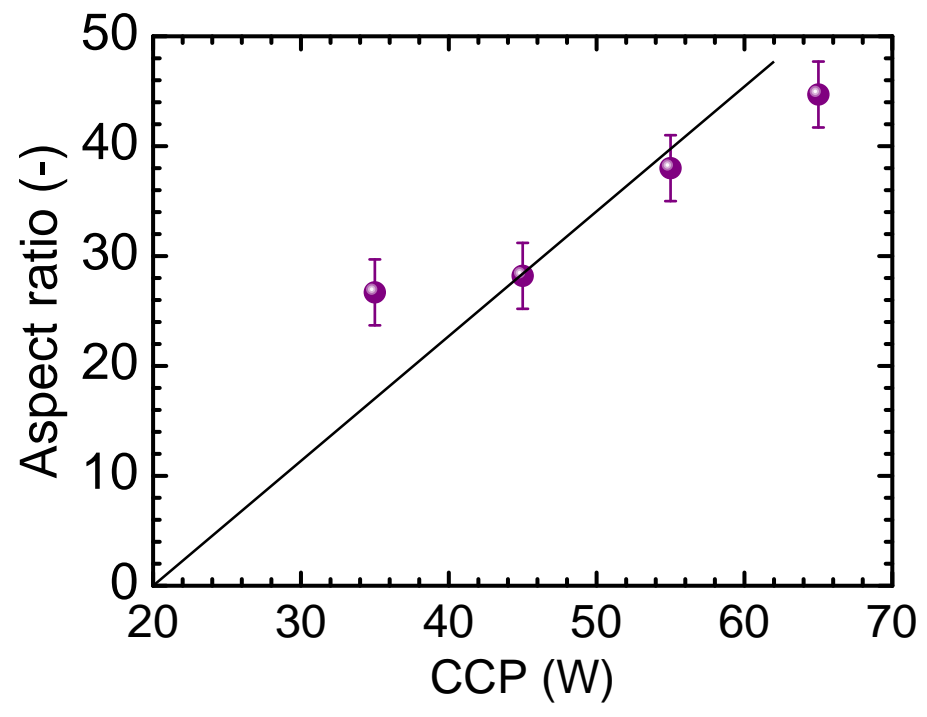

Figure 4.3: Aspect ratio dependence on the power of CCP plasma source. When the power is set to be ramped up from 30 to $40 \mathrm{~W}$ during etching cycle the data point is placed at the value of 25 on the plot. The straight line is a guide to the eye.

to the higher aspect ratio of etched structures. This is due to the increase in the ions energy when CCP power is increased. With the higher energy ions reach the bottom of the pores with higher kinetic therefore can reach deeper into the pores with sufficient energy for removing the protection layer. Tuning the CCP power even higher introduces a risk to reach the point when the physical sputtering of the hard mask occurs due to the extensive ion bombardment. Thus a CCP power ramp from 50 to $60 \mathrm{~W}$ was chosen as the optimum value for etching. Using an etching recipe listed in the Table 4.2 with the CCP power ramp from 50 to 60 allowed us to achieve the world record high aspect ratio nanopores in Si. We performed the series of experiments with different total etching time to determine the maximum aspect ratio that can be achieved with this parameters. Figure 4.4 shows the aspect ratio measured for different total etching times. Figure 4.5 shows the etching result with $21.4 \mu \mathrm{m}$ deep pores etched in Si with total etching time of 20 minutes. The diameter of one pore is $509 \mathrm{~nm}$ employing the aspect ratio as high as 42 . Up to our knowledge these are the highest aspect ratio pores etched in Si with DRIE. In 20 minutes there are 240 full cycles consisting of an one etching and one protection step. The large amount of particles visible on top 


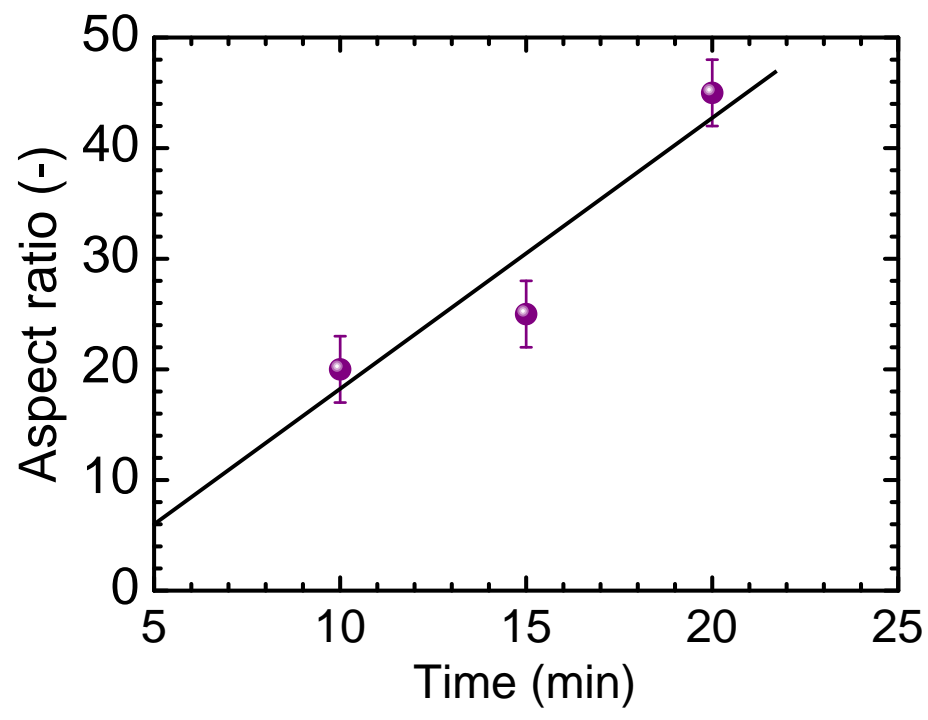

Figure 4.4: Aspect ratio dependence on the total etching time for the Bosch process described in Table 4.2. The straight line is guide to the eye.

of the pores is the result of the cleaving of a wafer.

\subsection{Advanced two-sided etching for 3D nanofabrication}

For fabrication of inverse woodpile structure (see Chapter 1, Figure 1.2) consisting of two perpendicular array of pores it is necessary to etch pores in two perpendicular directions. We chose to do so it two steps. After the etch mask is fabricated on two oblique faces of Si bar (see Chapter 2) one face of the Si bar is etched first then the sample is rotated by $90 \mathrm{deg}$ and second side is etched. This approach brings complications in the etching process since, firstly, the mask on the second etch side is present in the etching chamber during the first etching step and thus being affected by all the plasma components present in the chamber. Secondly, the when etching the second set on pores the first set of pores is already etched and so the etching is happening in the non-bulk but structured material. The present of a complicated structure in the material affect ions angular distribution inside the structure and thus affects the etching result. Thirdly, it is a complicated task to evaluate the etching result of a 3D structure since a precise cross sectioning is required to view the inner structure or a nondestructive method such as X-ray tomography described in the Chapter 5 of this thesis. The etching of $3 \mathrm{D}$ photonic crystals described in this section was done with two different etching machines. 


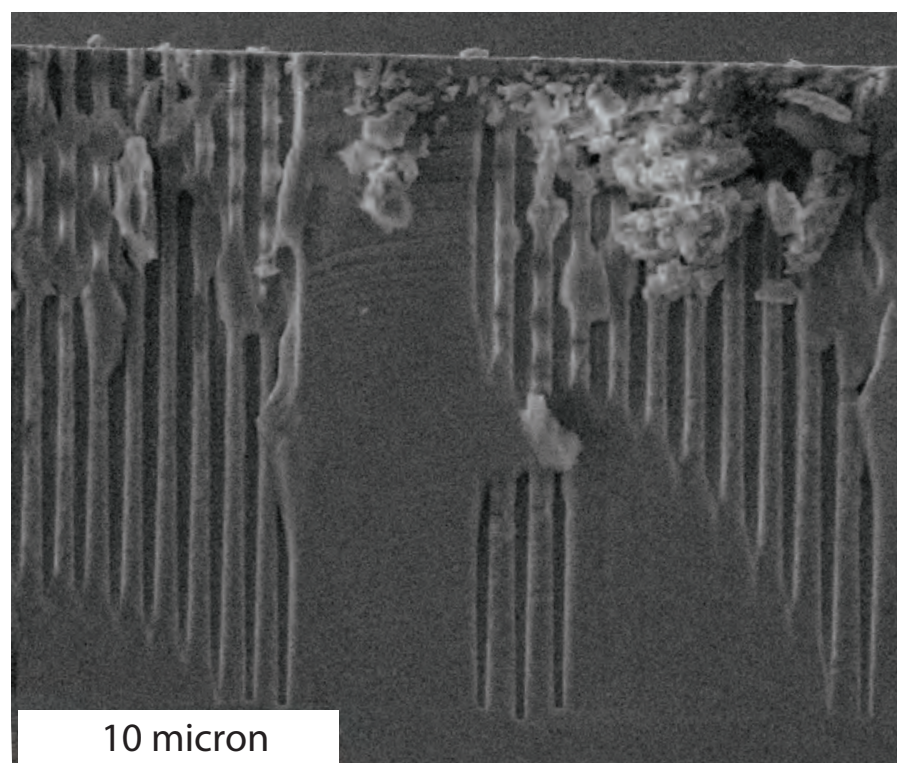

Figure 4.5: Ultra high aspect ratio pores etched with DRIE according to the process flow listed in Table $4.2 \mathrm{in} \mathrm{Si}$. The depth of $21.4 \mu \mathrm{m}$ and diameter of $510 \mathrm{~nm}$ yield the world record aspect ratio of 42 . 10 microns scale bar is shown on the picture. The particles on the surface are residuals from the cleaving.

\subsubsection{Choice of ICP power}

The process flow that was used for two-step etching of 3D inverse woodpile photonic crystals with Adixen was initially adopted from the Reference [7] as listed in the Table 4.5 with ICP power set to $I C P_{\text {high }}=1500 \mathrm{~W}$ during both etching and protection step. The etching time was set to 10 minutes (120 etching and protection cycles) as suggested by Reference [7]. The etching was performed twice: on one side of the Si bar and on the perpendicular side. The etching resulted in the typical for micro masking and strong over etching effect of nanowires all over the surface of Si bar and complete destruction of the structure. We associate this with ions bombardment of the etch mask especially aggravated because there two etching steps in total. Due to the ions bombardment $\mathrm{Cr}$ mask was physically sputtered and Cr particles spread over the chamber landing on the Si bar randomly providing micro masking effect. In Figure 4.6 we show the SEM image of the Cr mask (a) after 6 minutes of etching and (b) after 12 minutes of etching. It is clear from the images that after 12 minutes of etching (144 etching cycles) Cr mask is already significantly damaged and the diameter of individual apertures expands so much that the apertures are connected to each other. Naturally this implies that the structure etched under the mask after 12 minutes is also destroyed. To observe the evolution of the $\mathrm{Cr}$ etch mask depending 


\begin{tabular}{|c|c|}
\hline Parameter & Value \\
\hline$S F_{6}$ gas flow & $62 \mathrm{sccm}$ \\
\hline$C_{4} H_{8}$ gas flow & $200 \mathrm{sccm}$ \\
\hline Etching step duration & 3 seconds \\
\hline Deposition step duration & 2 seconds \\
\hline CCP power & $160 \mathrm{~W}$ \\
\hline Etching step ICP power & $I C P_{\text {high }}=1500 \mathrm{~W}$ \\
\cline { 2 - 2 } & $I C P_{\text {low }}=500 \mathrm{~W}$ \\
\hline \hline Deposition step ICP power & $1500 \mathrm{~W}$ \\
\hline
\end{tabular}

Table 4.3: The process flow for the etching of deep pores in Si with Adixen etcher.
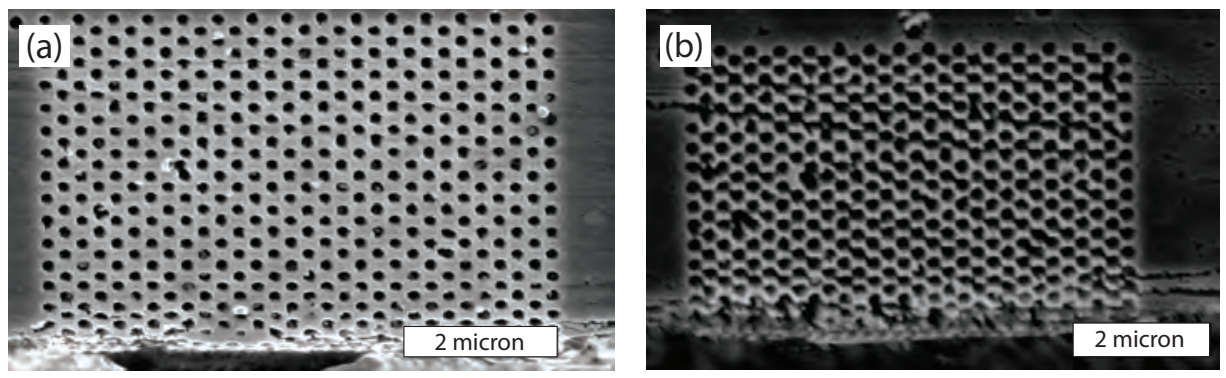

Figure 4.6: Evolution of the etch mask after 6 minuted (a) and 12 minutes (b) of etching with the Bosch process described in the Table 4.5 and a high ICP power $I C P_{\text {high }}=1500 \mathrm{~W}$.

on the total etching duration we performed a series of experiments where we measured the diameter of the apertures on the etch mask after each minute of etching. Figure4.7(a) shows the result for the etching process with ICP power $I C P_{\text {high }}=1500 \mathrm{~W}$. It is seen from the plot that aperture size on the mask level starts to steadily increase already after 8 minutes of etching. The smallest pitch between the pores in the design used for this experiment is $c=288 \mathrm{~nm}$ thus it is clear that when the aperture diameter increases to values larger that $277 \pm 15 \mathrm{~nm}$ such as at 12 minutes etch the apertures start to overlap. With the etching time more than 12 minutes the mask is destroyed thus it is not feasible to measure the aperture diameter anymore. From the plot in Figure 4.7(a) we conclude that the total etching duration with the set of parameters given in the Table 4.5 can not exceed 8 minutes. The depth of the pores $d$ depends strongly on the etching duration and for 8 minutes is determined from the cross sections to be only $d=3.2 \mu \mathrm{m}$. Therefore we choose to modify the recipe such that we can reduce the ion bombardment and physical sputtering of the mask in order to increase etching time. In order to reduce the risk of physics sputtering one can change the CCP or ICP power that control energy of ions or density of ions respectively $[12,13]$. The decrease of CCP power results in a poor directionality of ions and thus significant tapering of the pores [7]. Therefore we tune the ICP power down 


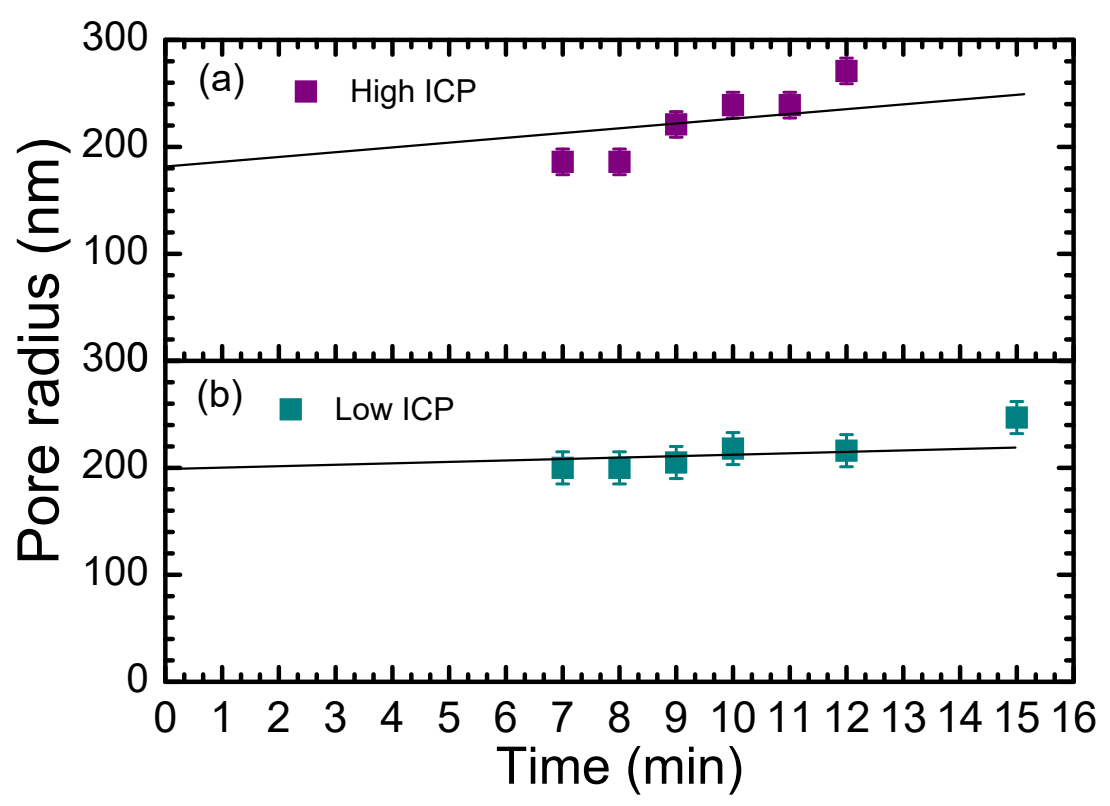

Figure 4.7: The pore radius on the etch mask depending on the total etching time with the Bosch process described in the Table 4.5 and high ICP power $I C P_{\text {high }}=1500 \mathrm{~W}$ (a) and low ICP power $I C P_{\text {low }}=500 \mathrm{~W}$.

during the etching step. Changing the ICP power may change the etch rate as well therefore we measured the etch rate at high ICP power $I C P_{\text {high }}=1500 \mathrm{~W}$ and low ICP power $I C P_{\text {low }}=500 \mathrm{~W}$. The etch rate measured from the cross sections of the pores is shown in Figure 4.8. It is seen that the etch rate drops by a factor of 2 from $0.4 \mu \mathrm{m} / \mathrm{min}$ to $0.2 \mu \mathrm{m} / \mathrm{min}$ with decrease of ICP power from 1500 to $500 \mathrm{~W}$. This change in the etch rate has to be taken into account when determining the total etching time required for the fabrication. Now let us consider the evolution of the etch mask for the Bosch process etching with parameters listen in Table 4.5 and low ICP power $I C P_{\text {low }}=500 \mathrm{~W}$. Figure 4.9 shows the view of the $\mathrm{Cr}$ etch mask (a) after 7 minutes of etching and (b) after 12 minutes of etching. It is clear from the comparison of two SEM images that after 12 minutes of etching with lower ICP power $\mathrm{Cr}$ mask is not damaged. We performed a series of experiments to determine the aperture size on the mask level depending on the total etching time. Figure 4.7(b) shows the aperture size measured on the $\mathrm{Cr}$ mask after the etching depending on the etching duration. It is seen from the plot that aperture diameter on the mask level changes insignificantly up to 10 minutes of etching then there is an increase in the aperture size up to $23 \%$ of its diameter and it stays nearly constant up to 25 minutes of etching. From the etch rate of $0.2 \mu \mathrm{m}$ determined in the Figure 4.8 for the 


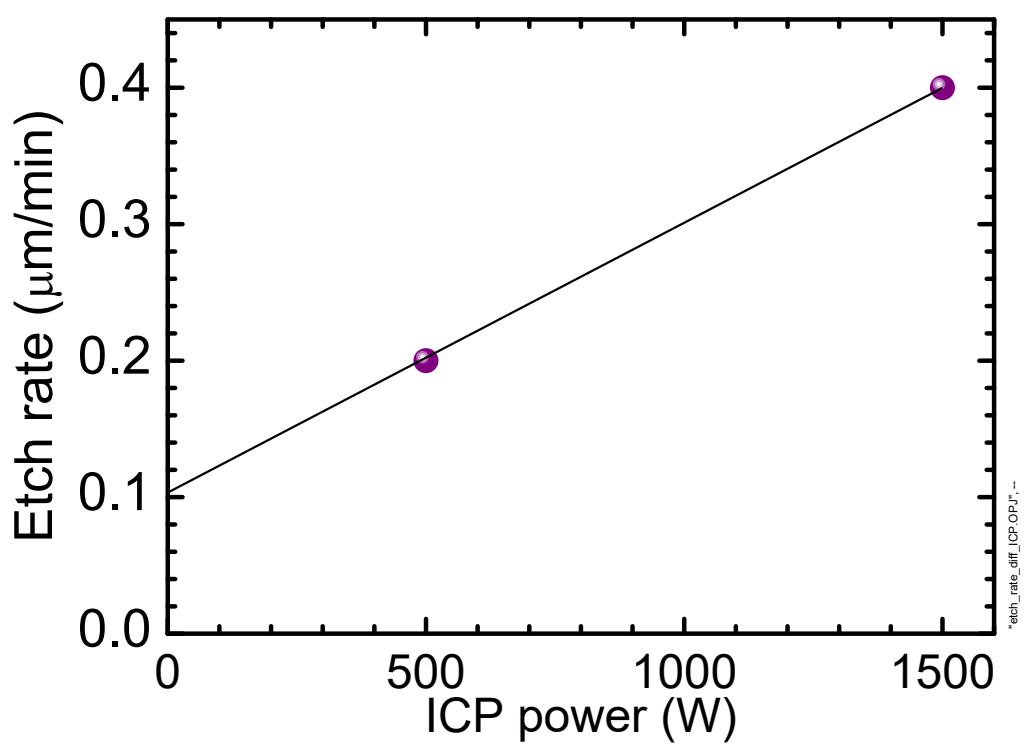

Figure 4.8: The etch rate dependence on the ICP power. Error bars are withing the symbol size. Straight line is a guide to the eye.
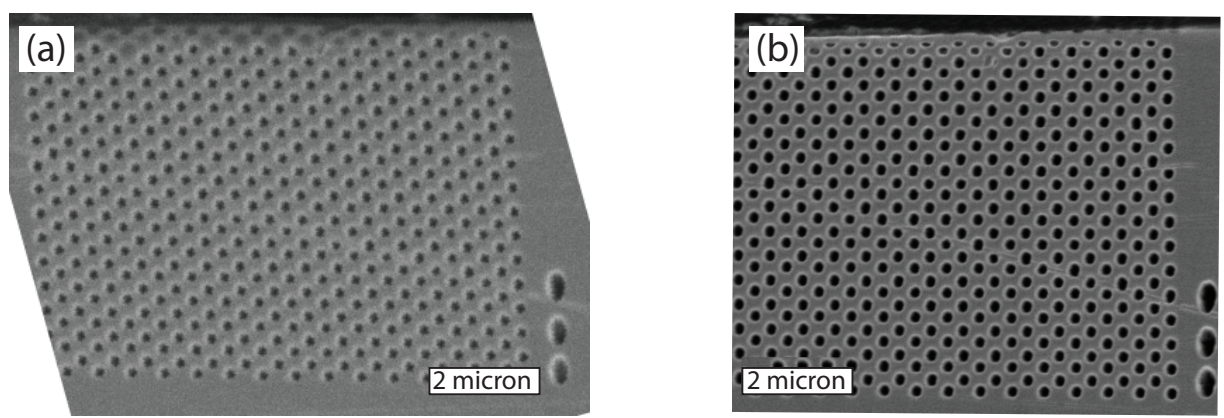

Figure 4.9: Evolution of the etch mask after 7 minuted (a) and 12 minutes (b) of etching with the Bosch process described in the Table 4.5 and low ICP power $I C P_{\text {low }}=$ $500 W$.

etching with low ICP power $I C P_{\text {low }}=500 \mathrm{~W}$ the depth of the pores $d$ after 25 minutes of etching is $d=5 \mu \mathrm{m}$. 


\subsubsection{Polymer residuals after the Bosch process}

It was observed during the fabrication that after the first side of the photonic crystal is etched with the Bosch process described above there is a thick layer of polymer present on the perpendicular face of the Si bar that is to be etched second. This layer originates from the protection step of the Bosch process since the process is design to cover vertical surfaces with protective polymer layer. Figure 4.10(a) shows the corner of Si bar after the etching of the first side. In the bottom of the image there is a thick blurred layer of polymer covering the second etch side. Naturally this layer must be removed before the second etching can be
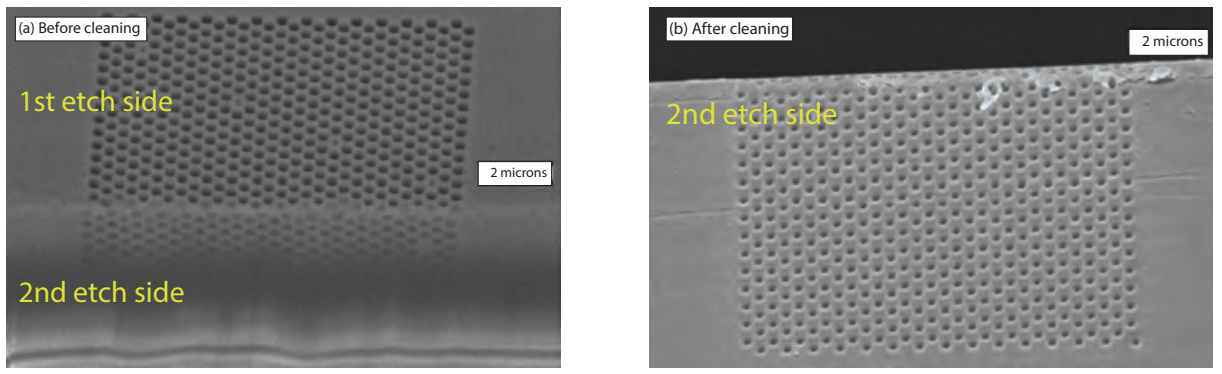

Figure 4.10: (a) The residuals of the polymer layer on the second etch side (bottom) after the Bosch process. The polymer layer is seen as thick blurred layer in the lower part of the image. (b) Second etch side after cleaning with oxygen plasma according to the process flow in Table 4.4. 2 microns scale bar is shown on the picture.

done. There are several possibilities to remove polymer residuals after the Bosch process. One of the standard approaches to remove polymer residuals from the surface is oxygen plasma stripping [14]. We developed a recipe for removal of the polymer layer from the second etch side using the same etcher as for the etching of the pores. The usage of the same equipment for several process steps decreases the amount of sample handling and transportation which is always preferable. The process flow for oxygen plasma cleaning in Adixen etching machine is listed in Table 4.4. The result of 5 minutes oxygen plasma cleaning according to the

\begin{tabular}{|c|c|}
\hline Parameter & Value \\
\hline $\mathrm{O}_{2}$ gas flow & $200 \mathrm{sccm}$ \\
\hline $\mathrm{CCP}$ power & 0 \\
\hline ICP power & $2000 \mathrm{~W}$ \\
\hline Substrate temperature & $-10 \mathrm{C}$ \\
\hline Time & $5 \mathrm{~min}$ \\
\hline
\end{tabular}

Table 4.4: The process flow for oxygen plasma cleaning in Adixen etching machine.

flow listed in Table 4.4 is shown in Figure 4.10(b). From the SEM image shown in Figure 4.10 it is seen that polymer layer is removed from the second etch side sufficiently to open the second side etch mask for further etching. Thus after the 
polymer layer is removed from the second etch side the sample is ready to be etched second time. We etch the second set of pores with the same recipe (see Table 4.5 with low ICP power) as the first set of pores. To evaluate the resulting structure we mill a cross section in the $X Y$ plane after two etching steps and view the inside of fabricated photonic crystal. The non-destructive inspection of $3 \mathrm{D}$ photonic crystals is presented in Chaper 5 of this thesis. The cross section view is shown in Figure 4.11. Two set of pores are clearly seen to be etched in perpendicular directions along $X$ and $Z$ axis. Due to the difficulty to precisely mill cross section parallel to $X Y$ plane there is a small tilt in the opened plane thus the pores running along $X$ direction are viewed under an angle. The inset in the Figure 4.11 shows the zoom into two crossing pores. From the zoomed image it is clearly seen that the pores are well aligned with the pores in $Z$ direction centered between pores in $X$ direction. Notably the zoom image reveals that no significant scalloping has occurred during the etching and the walls of the pores are smooth. From the cross section in Figure 4.11 we conclude that we

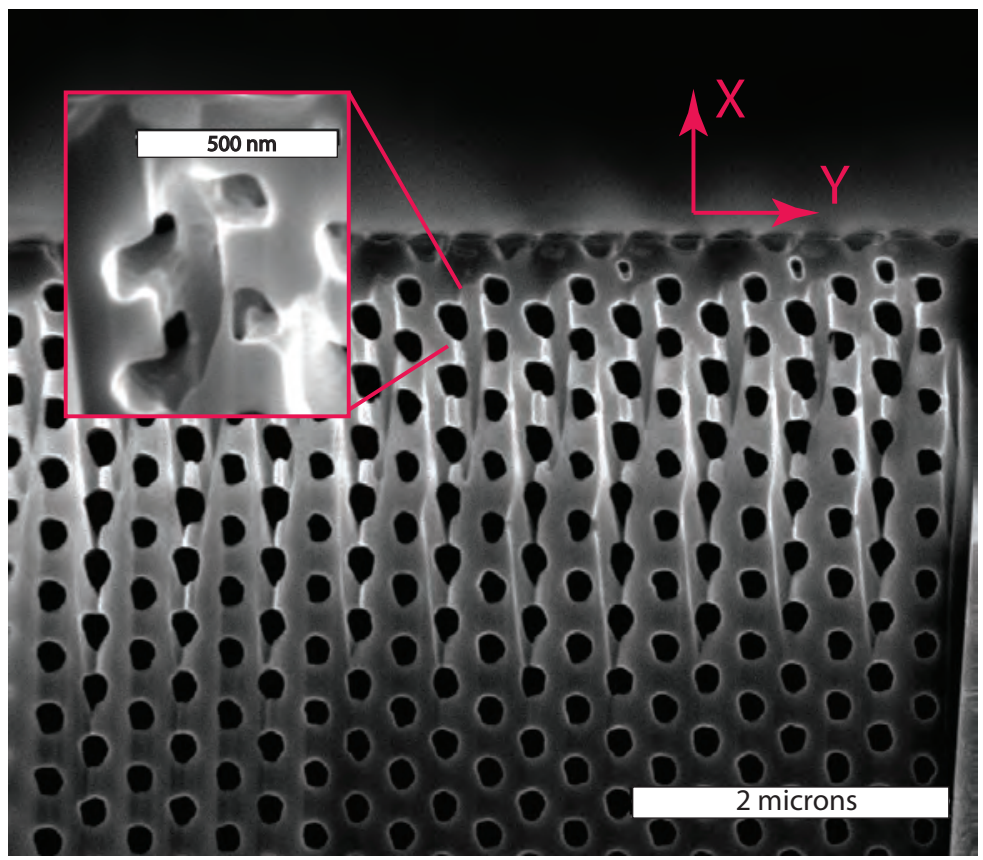

Figure 4.11: Cross section milled in $X Y$ plane through the 3D inverse woodpile photonic crystal after two consecutive etching steps. The inset shows zoom image taken at the higher resolution into the pores where the alignment of pores in $X$ and $Z$ direction is clearly seen as well as the smooth sidewalls of the pores.

have successfully etched 3D inverse woodpile photonic crystal structures with two step DRIE process using Adixen etcher. 2D photonic crystal samples and Sample $E$ that are studied by microscopic optical reflectivity in Chapter 6 and listed in the appendix B were fabricated using the process flow described in this 
section.

\subsubsection{Process flow transfer to SPTS Pegasus etcher}

Due to the technical reasons the process flow for 3D photonic crystal fabrication had to be transferred to the more advanced etcher SPTS Pegasus (shortly SPTS). Due to the differences in the etching machines some changes in the process flow were made. In particular the CCP power was tuned down to be ramp from 50 to $60 \mathrm{~W}$ as was described earlier in the section 4.4 and ICP power was set to $1500 \mathrm{~W}$ during both etching and deposition steps. The resulting process flow is listed in Table 4.2. The oxygen plasma cleaning recipe for the polymer residuals removal

\begin{tabular}{|c|c|}
\hline Parameter & Value \\
\hline $\mathrm{SF}_{6}$ gas flow & $62 \mathrm{sccm}$ \\
\hline $\mathrm{C}_{4} \mathrm{H}_{8}$ gas flow & $200 \mathrm{sccm}$ \\
\hline Etching step duration & 3 seconds \\
\hline Deposition step duration & 2 seconds \\
\hline CCP power etching step & ramp 50 to $60 \mathrm{~W}$ \\
\hline CCP power deposition step & $0 \mathrm{~W}$ \\
\hline ICP power etching step & $1500 \mathrm{~W}$ \\
\hline ICP power deposition step & $1500 \mathrm{~W}$ \\
\hline
\end{tabular}

Table 4.5: The process flow for the 3D photonic crystal fabrication with SPTS etcher.

on the second etch side was as well transferred to the SPTS etcher using the standard oxygen plasma chamber cleaning recipe as the basis. The process for cleaning of the second etch side before processing is listed in Table 4.6. Photonic

\begin{tabular}{|c|c|}
\hline Parameter & Value \\
\hline $\mathrm{O}_{2}$ gas flow & $200 \mathrm{sccm}$ \\
\hline $\mathrm{CCP}$ power & 0 \\
\hline ICP power & $1500 \mathrm{~W}$ \\
\hline Substrate temperature & $20 \mathrm{C}$ \\
\hline Time & $5 \mathrm{~min}$ \\
\hline
\end{tabular}

Table 4.6: The process flow for oxygen plasma cleaning in SPTS etcher.

crystal samples A, B, C, D studied in Chapters 5 and 6 were fabricated using DRIE process on SPTS etcher.

\subsection{Summary}

In this Chapter we described the process flow for fabrication of ultra high aspect ratio $2 \mathrm{D}$ nanopores in $\mathrm{Si}$ with the aspect ratio up to 42 . For fabrication of $3 \mathrm{D}$ photonic crystals we considered two process flows with high and low ICP powers powers and chose the one providing the deepest pores yet not destroying the etch 
mask by physical sputtering. We addressed the issue of residual polymer layer deposition on the second etch face of the mask for 3D photonic crystal fabrication. We provide the oxygen plasma cleaning recipe for polymer layer removal. We successfully fabricate inverse woodpile 3D photonic crystal structures and provide the cross section view on the etched pores. From the cross section we observe that the structure geometry matches the design and show the little amount of roughness on the sidewalls of the pores. 



\section{Bibliography}

[1] Y.Nishi and R.Doering Handbook of Semiconductor Manufacturing Technology Marcel Dekker. New York, (2000) 59

[2] B. Wu, A. Kumar, and S. Pamarthy High aspect ratio silicon etch: A review J. Appl. Phys. 108, 051101 (2010) 59, 60

[3] ] K. R. Ryan and I. C. Plumb A model for the etching of silicon in SF6/O2 plasmas, Plasma Chemistry and Plasma Processing 10, 207 (1990) 60

[4] F. Karouta A practical approach to reactive ion etching, J. Phys. D: Appl. Phys., 47 233501:1-14 (2014) 60, 62

[5] R.J. Shul and S. J. Peatron Handbook of Advanced Plasma Processing Techniques Springer (2000) 62

[6] L. A. Woldering, R. W. Tjerkstra, H. V. Jansen, I. D. Setija, and W. L. Vos Periodic arrays of deep nanopores made in silicon with reactive ion etching and deep UV lithography Nanotechnology 19 145304: 1-11 (2008) 63

[7] L. A. Woldering Fabrication of Photonic Crystals and Nanocavities $\mathrm{PhD}$ thesis, (2008) 61, 62, 63, 66, 67

[8] F. Laermer and A. Schilp Method of anisotropically etching silicon, U.S. Patent 5501893 (1996) 60

[9] K.Kitano, K. Suzuki, K. Ishizaki, and S. Noda Three-dimensional photonic crystals fabricated by simultaneous multidirectional etching Phys. Rev. B 91,155308 (2015) 59

[10] P. Latawiec, M. J. Burek, Y.-I. Sohn, and M. Loncar Faraday cage angledetching of nanostructures in bulk dielectrics J. of Vac. Sci. and Tech. B 34 041801 (2016) 59

[11] J. M. van den Broek, L. A. Woldering, R. W. Tjerkstra, F. B. Segerink, I. D. Setija, and W. L. Vos Inverse-woodpile photonic band gap crystals with a cubic diamond-like structure made from single-crystalline silicon Adv. Func. Mater. 22, 25-31 (2012) 60

[12] Y. M. Im, Y. B. Hahn, and S. J. Pearton Level set approach to simulation of feature profile evolution in a high-density plasma-etching system, J. Vac. Sci. Technol. B 19, 701 (2001) 67

[13] M. Boufnichel, S. Aachboun, F. Grangeon, P. Lefaucheux, and P. Ranson, Profile control of high aspect ratio trenches of silicon. I. Effect of process parameters on local bowing, J. Vac. Sci. Technol. B 20, 1508 (2002) 67

[14] W.M. Moreau Semiconductor Lithography: Principles, Practices, and Materials, Plenum Press, New York and London (2008) 70 



\section{CHAPTER 5}

\section{Looking inside 3D photonic nanostructures with X-ray tomography}

To critically assess the performance of three-dimensional (3D) photonic nanostructures, it is vital to view their internal structure. To this end, we have performed synchrotron X-ray tomography on diamondlike photonic band gap crystals made from silicon. In this chapter we describe the scientific background and experimental details of zoom Xray nanotomography for $3 \mathrm{D}$ imaging of photonic nanostructures. As the result of data analysis we obtain the real space $3 D$ density distribution of a number of complete photonic crystals with as low as 10 nanometer resolution. We also observe a number of hitherto buried deviations from the initial structure design that help to shed new light on optical performance.

\subsection{Introduction}

Three-dimensional (3D) photonic nanostructures are drawing a fast-growing attention for their advanced functionalities such as cloaking [1], metamaterials [2, $3]$, and photonic band gaps [4-6]. The optical properties of photonic nanostructures are in essence determined by their complex internal structure that typically consist of 3D arrays (periodic, aperiodic, random) of spheres, rods, pores, split-ring resonators, and other structural units [2-5]. Inevitably, a fabricated structure differs from the initial design, both systematically in case of structural deformations $[7,8]$, and statistically in case of size polydispersity and random positional disorder $[9,10]$. Consequently, the observed performance differs from the designed one. It is therefore critical to assess the structure of a 3D material and verify how well it matches the design.

In case of one-dimensional or two-dimensional nanostructures, the dimensionality is sufficiently low to assess the detailed structure with 2D planar imaging techniques such as scanning electron microscopy (SEM) [11], or atomic force, or scanning tunneling microscopy[12]. In case of 3D materials, one supplements SEM with micromachining or ion beam milling [13] to slice away part of the nanostructure in order to view inside. Unfortunately, however, this approach is destructive and irreversible, moreover milling damage may modify the viewed structure compared to the true structure. Alternatively, transmission electron 
microscopy (TEM) is used for 3D imaging with high resolution, however, since the required sample thickness is typically less than one micron [14], it is not suited for 3D photonic nanostructures.

For in situ and non-destructive structural characterization of 3D nanostructured materials, X-ray techniques are ideal in view of the high penetration of X-rays, and the short wavelength. Small-angle X-ray scattering (SAXS) is widely used to study 3D nanoparticle arrays [15]. Due to the statistical averaging, however, it is difficult to obtain information about local nano-sized features from SAXS [16, 17]. A powerful technique to study 3D structures with nanometer resolution is X-ray tomography that yields the real space $3 \mathrm{D}$ distribution of the material density $[18,19]$. In traditional tomography, the contrast is provided by the sample absorption that is simply related to the brightness of the transmitted image or radiograph [20]. Nevertheless, silicon and similar materials that are prevalent in nanophotonics and in CMOS industry are so weakly absorbing that other tomographic methods are required. For such weakly absorbing samples the real space structural information can be obtained from the optical phase change of an X-ray beam that propagates through the sample. Since the phase change of the beam depends on the real part of the refractive index, this method is even suitable for weakly absorbing samples.

\subsection{Zoom nanotomography}

\subsubsection{General scheme}

In order to improve spatial resolution from the micron scale in previously existing tomography techniques [21] to the nanometer scale, a hard x-ray projection microscopy or zoom tomography is employed [22-24]. The schematic of a projection microscopy is shown in Figure 5.1. The main difference from the conventional tomography scheme is that a sample is set at a small distance $z_{s}$ downstream of the focus and therefore magnified Fresnel diffraction patterns are recorded at the distance $z_{d}$ from the sample. The experimental parameters are given in section 5.3.2. Changing the out of focus distance $z_{s}$ allows to vary the magnification of the diffraction patterns, but at the same time will modify the pattern, as in Fresnel regime the diffraction pattern depends on the propagation distance, as is seen from our calculations in Appendix A. While focusing, the sample is illuminated with a spherical wave which relates to a plane wave illumination with an equivalent propagation distance $D$ and a magnification $M$ equal to [25]

$$
D=\frac{z_{s} z_{d}}{z_{s}+z_{d}}, M=\frac{z_{s}+z_{d}}{z_{s}}
$$

For each sample position $z_{s}$ the projection radiographs are recorded for a number of angles in the order of $10^{3}$ within $180^{\circ}$. Before a three-dimensional reconstruction can be applied, the collected radiographs must undergo three preprocessing steps to obtain projections of the sample. 


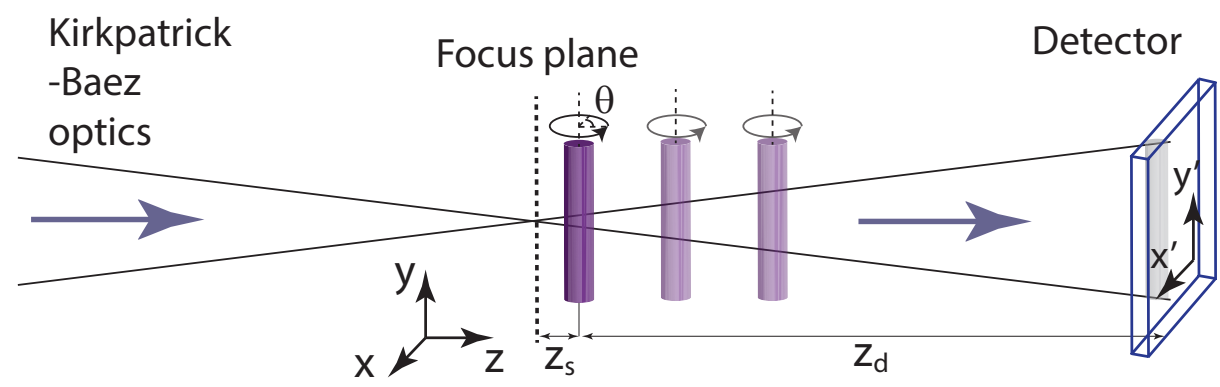

Figure 5.1: A scheme of the zoomed-in tomography setup using focused beam [24]. The x-ray beam is focused using Kirkpatrick-Baez (KB) optics [26] into a $20 \times 30 \mathrm{~nm}^{2}$ size focus. The sample is placed at multiple distances $z_{s}$ from the focus point and $z_{d}$ from the detector.

A first step is a correction related to the deviations with respect to a spherical wave of the sample illumination. The correction is implemented in a following way: two-dimensional phase grids are used as a calibration samples. The grids are imaged at the same position as the sample. Since real positions of maxima and minima of the grid image are known, the angular displacements of measured extrema to the calculated ones are determined. This information is used to undistort the radiographs [24].

The second preprocessing step is related to imperfections of Kirkpatrick-Baez (KB) optics. Due to the surface roughness of the KB mirrors, the incoming wave has non-flat phase profile. In order to correct for this fact, extra images besides an object image $I^{\text {object }}$ are taken. One image is taken without an object and is called flat image $I^{\text {flat }}$ and another image is taken without the beam and is called dark image $I^{\text {dark }}$. The dark image must be subtracted from all measured intensities including the flat one and then the intensity measured in presence of the object are normalized to the flat intensity:

$$
I_{\text {exp }}=\frac{I^{\text {object }}-I^{\text {dark }}}{I^{\text {flat }}-I^{\text {dark }}},
$$

Another way to get rid of distortions introduced by KB optics is to randomly slightly change the sample position at every measured distance. In this way the position of the artifacts introduced by $\mathrm{KB}$ optics is also changed and therefore averages out in later reconstruction steps.

The third preprocessing step is to bring the raw images to the same magnification and origin using Equations 5.1. After all preprocessing corrections are done, the in-line holograms of the sample are obtained as in the parallel beam case. There the phase retrieval algorithm is applied [21], see section 5.2.2. 


\subsubsection{Phase retrieval}

A special phase retrieval algorithm is required to distinguish the phase and attenuation modulations introduced by the object. Let us consider an object with a transmission function

$$
T(x, y)=\exp [-B(x, y)] e^{[j \phi(x, y)]}
$$

that is illuminated with an incident monochromatic X-ray beam $u_{\text {inc }}(x, y)$ propagating along the $Z$ axis, where

$$
\begin{gathered}
B(x, y)=\frac{2 \pi}{\lambda} \int \beta(x, y, z) d z \\
\phi(x, y,)=\frac{2 \pi}{\lambda} \int[1-\delta(x, y, z)] d z=\phi_{a i r}-\frac{2 \pi}{\lambda} \int \delta(x, y, z) d z,
\end{gathered}
$$

and where $\delta$ is the real part and $\beta$ is the imaginary part of the refractive index $n$ described in Equation A.3. The field directly behind the object is equal to

$$
u_{0}(x, y)=T(x, y) \cdot u_{i n c}(x, y) .
$$

To obtain the field at a distance $z$ behind the sample we use the same approach as in section A.7.2. In this approach the field at a distance $z$ from the object is a convolution of the field at $z=0$ and the Fresnel transfer function. The convolution in real space corresponds to a multiplication in Fourier space, therefore from now on we will consider field and intensity in the Fourier domain $\left(f_{X}, f_{Y}\right)$

$$
u_{z}\left(f_{X}, f_{Y}\right)=H\left(f_{X}, f_{Y}\right) \cdot u_{0}\left(f_{X}^{2}+f_{Y}^{2}\right),
$$

with Fresnel transfer function $H$ from equation A.45. The intensity of the field is equal to $I(x, y)=|u(x, y)|^{2}$, or, at the distance $z$ in the Fourier domain $\check{I}_{z}\left(f_{X}, f_{Y}\right)=\left(u_{z} \star u_{z}^{*}\right)$. From now on symbols with a tilde indicate a Fourier transform. Here we limit the equation to a one-dimensional case as in References $[27,28]$.

$$
\begin{array}{r}
\check{I}_{z}(f)=\left(u_{z} \star u_{z}^{*}\right)_{f}= \\
=e^{(-j \pi \tau f)} T(\tau) \star e^{(j \pi \tau f)} T^{*}(\tau)= \\
=\int_{-\infty}^{\infty} e^{(-j \pi \eta f)} T(\eta) e^{(j \pi(\tau-\eta) f)} T^{*}(\tau-\eta) d \eta= \\
=e^{-j \pi \lambda z f^{2}} \int_{-\infty}^{\infty} e^{j 2 \pi \eta f} T(\eta) T^{*}(\eta-\lambda z f) d \eta
\end{array}
$$

In the derivation we change variable to $\lambda z f=\tau$ and use the definition of a convolution:

$$
(g(t) \star f(f)) \equiv \int_{-\infty}^{\infty} g(\tau) f(t-\tau) d \tau,
$$

To equation 5.8 we now apply the slowly varying phase (SVP) approximation [28]. Supposing

$$
|\phi(\eta)-\phi(\eta-\lambda z f)|<<1
$$


we obtain under the integral in equation 5.11

$$
T(\eta) T^{*}(\eta-\lambda z f) \approx 1+j[\phi(\eta)-\phi(\eta-\lambda z f)] .
$$

Neglecting the absorption $(B<<\phi)$ and substituting 5.14 into equation 5.11 we simplify 5.11 to

$$
\begin{aligned}
& \check{I}_{z}(f)=e^{-j \pi \lambda z f^{2}} \int_{-\infty}^{\infty} e^{j 2 \pi \eta f}(1+j[\phi(\eta)-\phi(\eta-\lambda z f)]) d \eta=(5.15) \\
& =\int_{-\infty}^{\infty} e^{-j \pi \lambda z f^{2}} e^{j 2 \pi \eta f} d \eta+\int_{-\infty}^{\infty} e^{-j \pi \lambda z f^{2}} e^{j 2 \pi \eta f} j[\phi(\eta)-\phi(\eta-\lambda z f)] d \eta=(5.16) \\
& =\delta(f)+\int_{-\infty}^{\infty} j e^{-j \pi \lambda z f^{2}} e^{j 2 \pi \eta f} \phi(\eta) d \eta-\int_{-\infty}^{\infty} j e^{-j \pi \lambda z f^{2}} e^{j 2 \pi \eta f} \phi(\eta-\lambda z f) d \eta=(5.17) \\
& =\delta(f)+j e^{-j \pi \lambda z f^{2}} \check{\phi}(f)-j e^{j \pi \lambda z f^{2}} \check{\phi}(f)=(5.18) \\
& =\delta(f)+2 \sin \left(\pi \lambda z f^{2}\right) \check{\phi}(f)(5.19)
\end{aligned}
$$

where $\check{\phi}$ is the Fourier transform of $\phi(\eta)$. Equation 5.19 shows a linear relationship in reciprocal space between the observed intensity $\check{I}_{z}(f)$ and the pursued phase $\check{\phi}(\eta)$, also found in Reference [29].

Taking into account the finite source size (therefore finite coherence $\gamma_{c}$ ), as well as the detector's point spread function $R$ causes the real intensity measured in the experiment to differ from the theoretical one by multiplication in reciprocal space (convolution in real space) of above mentioned characteristics:

$$
\check{I}_{z}^{\text {exp }}(f)=\check{I}_{z}^{\text {theory }}(f) \cdot \check{R}(f) \cdot \gamma_{c}(\lambda z f) .
$$

Since the result of equation 5.19 is obtained using the SVP approximation, the formula used to retrieve the phase information from the measured intensity is obtained by performing a least square minimization of the difference between the measured intensity and the approximated one. Let the cost function in reciprocal space be defined as

$$
S_{c} \equiv \frac{1}{N} \sum_{m=1}^{N} \int d f\left|\check{I}_{z_{m}}^{\text {exp }}(f)-\check{I}_{z_{m}}^{a p p r o x}\right|^{2} .
$$

The summation here is applied over multiple propagation distances $z_{m}$ where images are taken. Taking into account the result of derivation (5.19)

$$
\check{I}_{z_{m}}^{a p p r o x}(f)=\delta(f)+2 \sin \left(\pi \lambda z_{m} f^{2}\right) \check{\phi}(f)
$$

we find the minimum of equation 5.21 to occur when

$$
\begin{aligned}
& \frac{\partial S_{c}}{\partial \check{\phi}}=\frac{\partial\left[\left(\check{I}_{z_{m}}^{\text {exp }}\right)^{2}-2 \check{I}_{z_{m}}^{\text {exp }} \check{I}_{z_{m}}^{\text {approx }}+\left(\check{I}_{z_{m}}^{\text {approx }}\right)^{2}\right]}{\partial \check{\phi}}=0, \\
& \check{\phi}=\frac{\sum_{m} \check{I}_{z_{m}}^{\exp } \sin \left(\pi \lambda z f^{2}\right)}{\sum_{m} 2 \sin ^{2}\left(\pi \lambda z f^{2}\right)}
\end{aligned}
$$


Now admitting the presence of weak absorption $(B(x)<<1)$, described by the imaginary part of the sample's refractive index, we obtain an expression with an extra cosine term for the absorption [27]:

$$
\check{I}_{z}(f) \approx \delta(f)+2 \sin \left(\pi \lambda z f^{2}\right) \check{\phi}(f)-2 \cos \left(\pi \lambda z f^{2}\right) \cdot \check{B}(f)
$$

Applying the least square minimization approach in the same way as it is done above, the retrieval formulas for $\check{\phi}$ and $\check{B}$ are:

$$
\check{B}(f)=\frac{1}{2 \Delta}\left(A \cdot \sum_{m} \check{I}_{z_{m}}^{e x p} \sin \left(\pi \lambda z_{m} f^{2}\right)-K \cdot \sum_{m} \check{I}_{z_{m}}^{e x p} \cos \left(\pi \lambda z_{m} f^{2}\right)\right),
$$

and

$$
\check{\phi}(f)=\frac{1}{2 \Delta}\left(C \cdot \sum_{m} \check{I}_{z_{m}}^{e x p} \sin \left(\pi \lambda z_{m} f^{2}\right)-A \cdot \sum_{m} \check{I}_{z_{m}}^{e x p} \cos \left(\pi \lambda z_{m} f^{2}\right)\right),
$$

with the following coefficients:

$$
\begin{aligned}
A & =\sum_{m} \sin \left(\pi \lambda z_{m} f^{2}\right) \cos \left(\pi \lambda z_{m} f^{2}\right) \\
K & =\sum_{m} \sin \left(\pi \lambda z_{m} f^{2}\right)^{2} \\
C & =\sum_{m} \cos \left(\pi \lambda z_{m} f^{2}\right)^{2} \\
\Delta & =K C-A^{2} .
\end{aligned}
$$

Using equations (5.26) and (5.27) we obtain the object transmission function from the intensity distribution measured at several distances behind the sample. In practice there are few approaches to simplify the equations above that depend on prior knowledge about the sample.

\section{Phase retrieval approach 1: phase-only object}

Approach 1 is used in case the sample is purely a phase object. In this case the absorption term $\check{B}$ in equation (5.24) is neglected, therefore the relation between intensity and phase becomes

$$
\check{I}_{z}(f) \approx \delta(f)+2 \sin \left(\pi \lambda z f^{2}\right) \check{\phi}(f)
$$

Following the same procedure as shown in equations 5.20-5.23, the phase is found by using only one equation 5.23. This simplified approach has a difficulty when the denominator vanishes at zero frequencies $(f=0)$. In order to overcome this problem the regularization of equation 5.21 is needed. The simplest regularization is the Tikhonov regularization [30] when the energy of the solution is added to the cost function 5.21 with a coefficient $\alpha$ to be determined:

$$
S_{c} \equiv \frac{1}{N} \sum_{m=1}^{N} \int d f\left|\check{I}_{z_{m}}^{e x p}(f)-\check{I}_{z_{m}}^{a p p r o x}\right|^{2}+\alpha \int d f|\check{\phi}(f)|^{2}
$$


In this case the equation for the phase will be

$$
\check{\phi}=\frac{\sum_{m} \check{I}_{z_{m}}^{e x p} \sin \left(\pi \lambda z f^{2}\right)}{\sum_{m} 2\left(\sin ^{2}\left(\pi \lambda z f^{2}\right)+\alpha\right)}
$$

When a prior estimate of the phase of the object $\check{\phi}^{\text {prior }}$ is known, the regularization term is written as

$$
S_{c} \equiv \frac{1}{N} \sum_{m=1}^{N} \int d f\left|\check{I}_{z_{m}}^{\text {exp }}(f)-\check{I}_{z_{m}}^{\text {approx }}\right|^{2}+\alpha \int d f\left|\check{\phi}(f)-\check{\phi}^{\text {prior }}\right|^{2} .
$$

In this case the equation for the phase is

$$
\check{\phi}=\frac{\sum_{m} \check{I}_{z_{m}}^{\text {exp }} \sin \left(\pi \lambda z f^{2}\right)+\alpha \check{\phi}^{\text {prior }}}{\sum_{m} 2\left(\sin ^{2}\left(\pi \lambda z f^{2}\right)+\alpha\right)}
$$

The estimation of the $\check{\phi}^{\text {prior }}$ can be extracted from the prior knowledge about the sample.

\section{Phase retrieval approach 1.a: homogeneously absorbing object}

In case the absorption can not be neglected but the sample is homogeneous such that the ratio between real and imaginary parts of refractive index is constant over the sample

$$
\frac{\delta}{\beta}=\text { const. }
$$

one can use prior knowledge about the refractive index of the material to link the phase $\phi$ and attenuation $B(x, y)$ in equation 5.24. Phase and absorption terms can be written using $\beta$ and $\delta$ as shown in equations 5.4 and 5.5:

$$
\begin{aligned}
& B(x, y)=\frac{2 \pi}{\lambda} \int \beta(x, y, z) d z, \\
& \phi(x, y)=-\frac{2 \pi}{\lambda} \int \delta(x, y, z) d z .
\end{aligned}
$$

Now we consider $\phi$ and $B$ to be dependent and therefore we can express $B$ in terms of $\phi$ as

$$
B(x, y)=-\phi\left(\frac{\beta}{\delta}\right)
$$

With this assumption the equation for the intensity (5.24) becomes

$$
\check{I}_{z}(f) \approx \delta(f)+2\left[\sin \left(\pi \lambda z f^{2}\right)+\cos \left(\pi \lambda z f^{2}\right) \cdot \frac{\beta}{\delta}\right] \check{\phi}(f)
$$

This equation does not tend to zero in the low frequency limit, therefore there is no need for regularization. Nevertheless it is still necessary to measure the intensity for a number of distances $z_{m}$ since the combined term $\sin \left(\pi \lambda z f^{2}\right)+$ $\cos \left(\pi \lambda z f^{2}\right)$ can vanishes at certain frequencies $f$. In case of our 3D photonic crystal samples we neglect the spatial $\frac{\beta}{\delta}$ change inside the pores in order to use this approach. 


\section{Phase retrieval approach 2: non-homogeneous absorbing sample}

Another approach to phase retrieval is based on additional absorption measurements of the sample $I_{z=0}^{a b s}$. In this case an extra image of the sample is taken at a very close distance to the detector so that the influence of the phase contrast is minimal. After that the ratio between the intensity at every distance $z_{m}$ to the absorption image in real space is calculated $\frac{I_{z m}(x)}{I_{z=0}^{a b s}}$. This approach can not be used in case of zoom tomography since at the very close distance to the detector the magnification is equal to 1 and the resolution is equal to the resolution of the detector, therefore the gain of resolution due to magnification is lost.

\section{Phase retrieval approach 3: general dispersive and absorptive object}

In this general approach the phase $\phi$ and attenuation $B$ are considered to be totally independent and the full set of equations (5.25) -(5.27) is used for phase retrieval. This is the most complicated approach which is rarely used.

The group of above mentioned approaches are called contrast transfer function (CTF) methods. The contrast transfer function is a term $\sin \left(\pi \lambda z f^{2}\right)$ that appears in each of the approaches.

\section{Paganin approach to phase retrieval using the transport of intensity equation}

In addition to the CTF group of methods there are methods based on the transport of intensity equation (TIE) [31, 32]:

$$
\frac{\partial I(r, z)}{\partial z}=-\frac{\lambda}{2 \pi} \nabla(I(r, z) \nabla \phi(r, z))
$$

where $I(r, z)$ is the irradiance at the point $(x, y, z)$ defined as

$$
I_{z}(r) \equiv\left|u_{z}(r)\right|^{2}
$$

The wave amplitude can be expressed in terms of the irradiance $I$ and the phase $\phi$ as follows:

$$
u_{z}(r)=I_{z}(r)^{1 / 2} e^{[} i \phi_{z}(r) .
$$

To derive equation (5.37) let us consider light propagating in the $z$ direction and time-dependent wave amplitude is written as $u_{z}(r) e^{(-i 2 \pi \omega t)}$, where $r$ is a two-dimensional vector in the transverse direction. The amplitude then satisfies approximately the parabolic equation [32]

$$
\left(i \frac{\partial}{\partial z}+\frac{\nabla^{2}}{2 k}+k\right) u_{z}(r)=0
$$

where $\nabla^{2}=\left[\left(\partial^{2} / \partial x^{2}\right)+\left(\partial^{2} / \partial y^{2}\right)\right]$ and $k=2 \pi / \lambda$. This is shown in Reference [32] by direct substitution of Fresnel diffraction result (see equation A.19) in the appendix A. 
Let equation (5.40) be multiplied on the left side by $u_{z}^{*}$ and the complex conjugate of equation (5.40) be multiplied on the left side by $u_{z}$. The two resulting equations are subtracted, one gets

$$
\frac{2 \pi}{\lambda} \frac{\partial}{\partial z} I=-\nabla \cdot I \nabla \phi
$$

which is known as the transport-of-intensity equation. We first consider the case when the sample is illuminated with a collimated X-ray beam and therefore unit magnification. At the end of the derivation the modifications will be made for the non-collimated case. We also assume that the object under study is homogeneous. For normally incident plane-wave radiation of uniform intensity over the sample area, the intensity of the radiation right behind the sample $(z=0)$ is assumed to be approximated by Bouguer-Lambert-Beer's Law of absorption:

$$
I(r, z=0)=I^{i n} e^{(-\mu T(r))},
$$

where $T(r)$ is the projected thickness of the homogeneous object onto a plane where the image is taken, $\mu$ is the linear attenuation coefficient, and $I^{i n}$ is the uniform intensity of the incident radiation. If the object is sufficiently thin, the phase $\phi(r, z=0)$ of the illuminating beam right behind the sample is proportional to the projected thickness:

$$
\phi(r, z=0)=-\frac{2 \pi}{\lambda} \delta T(r)
$$

We substitute equations (5.42) and (5.43) in the transport of intensity equation (5.41) to we obtain:

$$
\frac{2 \pi}{\lambda} \frac{\partial}{\partial z} I(r, z=0)=\nabla \cdot\left(I^{i n} e^{(-\mu T(r)} \nabla \frac{2 \pi}{\lambda} \delta T(r)\right)
$$

where using the equality

$$
\left.\delta \nabla \cdot e^{(-\mu T(r))} \nabla T(r)=-\frac{\delta}{\mu} \nabla^{2} e^{(-\mu T(r)}\right)
$$

equation (5.44) is written as

$$
\frac{\partial}{\partial z} I(r, z=0)=\frac{\delta}{\mu} I^{i n} \nabla^{2} e^{(-\mu T(r))} .
$$

Let us estimate the left side of equation (5.46) using the intensity measurements in two sufficiently closely spaced planes separated by a distance $R_{2}$ :

$$
\frac{\partial}{\partial z} I(r, z=0) \approx \frac{I\left(r, z=R_{2}\right)-I(r, z=0)}{R_{2}}=\frac{I\left(r, z=R_{2}\right)-e^{(-\mu T(r))} I^{i n}}{R_{2}} .
$$

Substituting equation 5.47 into equation 5.46 and re-arranging, gives

$$
\left(-\frac{R_{2} \delta}{\mu} \nabla^{2}+1\right) e^{(-\mu T(r))}=\frac{I\left(r, z=R_{2}\right)}{I^{\text {in }}} .
$$


To proceed we represent both images at the distance $z=0$ and at the distance $z=R_{2}$ as Fourier integrals:

$$
\left\{\begin{array}{l}
I^{i n} e^{(-\mu T(r))}=\frac{I^{i n}}{2 \pi} \iint F\left\{e^{(-\mu T(r))}\right\} e^{(i k r)} d k \\
I\left(r, z=R_{2}\right)=\frac{1}{2 \pi} \iint F\left\{I\left(r, z=R_{2}\right)\right\} e^{(i k r)} d k
\end{array}\right.
$$

Substituting into equation (5.48) gives

$$
F\left\{e^{(-\mu T(r))\}}=\mu \frac{F\left\{I\left(r, z=R_{2}\right)\right\} / I^{i n}}{R_{2} \delta|k|^{2}+\mu}\right.
$$

Taking the inverse Fourier transform of equation (5.50) and solving for $T(r)$, we arrive at:

$$
T(r)=-\frac{1}{\mu} \log _{e}\left(F^{-1}\left[\mu \frac{F\left\{I\left(r, z=R_{2}\right)\right\} / I^{i n}}{R_{2} \delta|k|^{2}+\mu}\right]\right) .
$$

To conclude the derivation we need to remove the assumption of a collimated beam. To do so we take into account the magnification factor $M$ given earlier in equation (5.1):

$$
T(r)=-\frac{1}{\mu} \log _{e}\left(F^{-1}\left[\mu \frac{F\left\{M^{2} I\left(M r, z=R_{2}\right)\right\} / I^{i n}}{R_{2} \delta|k|^{2} / M+\mu}\right]\right) .
$$

Equation (5.52) shows how to solve the transport-of-intensity equation (5.37) for the projected thickness $T(r)$ of the sample. The phase of the radiation at the exit surface of the sample can be found by substituting the derived equation into equation (5.43).

The approach above is more exact in case of strong absorption, although in the limit of high frequencies the solution is not converging. This method can be used in order to find a good estimation of $\phi^{\text {prior }}$ for iterative phase retrieval approaches.

\subsection{Experimental}

\subsubsection{Samples}

As a first example of a 3D nanophotonic structure viewed by zoom tomography, we focus on 3D silicon photonic band gap crystals [33]. The density distribution $\rho_{e}(X, Y, Z)$ of the inverse woodpile structure is designed as two perpendicular two-dimensional (2D) arrays of pores, as discussed in Chapter 1.

Three 3D photonic crystal samples were chosen for tomography experiments. For the ease of reading samples are named $\mathbf{A}, \mathbf{B}$ and $\mathbf{C}$ here. The corresponding names of the samples in the lab are given in Table B in Appendix B. Samples A and $\mathbf{B}$ are $3 \mathrm{D}$ photonic crystal samples that were fabricated with the two-step etch mask process. The detailed description of the fabrication process is given in Reference [36]. Typical SEM image of the crystal fabricated with two-step etch mask approach is shown in Figure 5.2. The fabrication of the 3D photonic crystal 
with two-step mask approach starts with the complete Si wafer where deep pores are etched through the chromium mask filling the entire wafer. The wafer is then cleaved to open a perpendicular face for the second set of pores. The mask for the second array of pores is defined using focused ion beam lithography (FIB) and thus limited to be $10 \times 10 \mu \mathrm{m}^{2}$ by the FIB field of view. Thus the extent of the $3 \mathrm{D}$ structure where both sets of pores are overlapping is at most $10 \times 10 \mu \mathrm{m}^{2}$ and there is a large 2D array of pores surrounding the $3 \mathrm{D}$ structure as seen in the Figure 5.2. Sample A was additionally kept in a quantum dot suspension for spontaneous emission measurements [35].

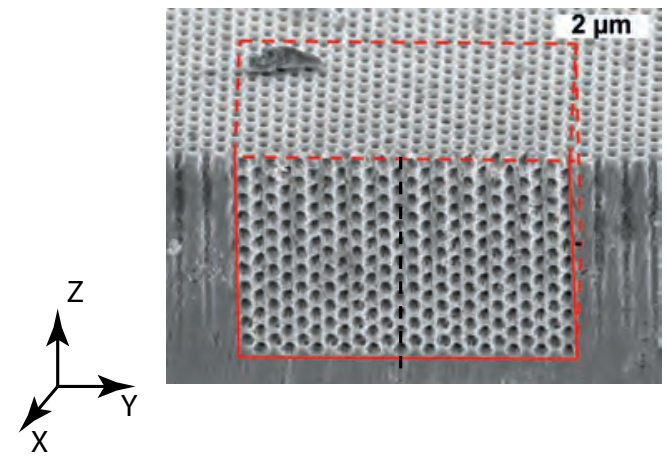

Figure 5.2: Typical SEM image of the external surface of a sample fabricated in the same way as samples $\mathbf{A}$ and $\mathbf{B}$. Two sets of pores are running in $Z$ and $X$ directions. The 3D crystal's estimated extent is delimited by the red dashed lines. The 3D crystal in surrounded by a large array of $2 \mathrm{D}$ pores running in $Z$ direction due to the properties of a fabrication process. The scale bar is shown.

Sample A was studied in three different experiments. First, the sample was measured with $20 \mathrm{~nm}$ resolution. In a second measurement a small random displacement of the sample position was applied during the rotation. This is a step performed to average out beam artifacts with a fixed position on the detector as is described in section 5.2.1. The third measurement on this sample was performed with $10 \mathrm{~nm}$ resolution to compare to the experiment with $20 \mathrm{~nm}$ resolution.

Sample B was measured once with a resolution of $20 \mathrm{~nm}$.

Sample $\mathbf{C}$ is an inverse woodpile 3D photonic crystal sample that was fabricated on a silicon bar using the novel single step etch mask approach described in chapter 2. A typical SEM image of the sample fabricated with a single step etch mask is shown in Figure 2.1 in chapter 2. The 3D structure is located on the edge of the silicon bar and surrounded by bulk silicon and air. The sample was measured with $10 \mathrm{~nm}$ resolution. 


\subsubsection{Experimental setup of beamline}

X-ray tomography experiments were performed in collaboration with the team guided by P. Cloetens at the European Synchrotron Radiation Facility (ESRF), beamline ID16A [37]. The beamline has the possibility to focus x-ray beam using Kirkpatrick-Baez optics down to 20 by $30 \mathrm{~nm}^{2}$ size and operate in a photon energy range between 17 and $33.6 \mathrm{keV}$. During all our experiments the photon energy of the beam was $17 \mathrm{keV}$ that corresponds to the wavelength $\lambda=0.07 \mathrm{~nm}$. Every sample was measured at 4 different sample to detector distances $z_{d}$, see Figure 5.1 and section 5.2.2. At each sample position between 1500 and 1800 images were recorded while rotating the sample from 0 to 180 degrees. For every image the exposure time was set to 0.3 seconds. The rotation axis was chosen to be approximately 12 micron deep inside the silicon.

The experimental data are collected as sets of transmission radiograms for every rotation angle $\theta$ and every distance $z_{d}$. A typical transmission radiogram from our sample is shown in Figure 5.3. There are three regions distinguished on the radiogram, firstly, at the top of the image there are black and white lines which are the result of Fresnel diffraction on the edge of the sample. Secondly, in the region indicated with the yellow rectangle the $3 \mathrm{D}$ structure is located. Thirdly, below the silicon to air interface the sample consist of a periodic array of pores in silicon that are oriented along the propagation of the x-ray beam, therefore in transmission such a structure results in the periodic pattern. The period of the pattern is $480 \mathrm{~nm}$ which corresponds to the period of $2 \mathrm{D}$ photonic array. The images taken at different sample to detector distances have different magnification and due to imperfections of mechanical translation are not always well aligned. Therefore the first step in data analysis is to bring all the images taken at one angle $\theta$ to the same magnification and align them with respect to each other. Typically translation shifts between images are calculated for every 100 images and then fitted with smooth curves to obtain shifts for remaining images. The lateral shift for every 100 images can be calculated automatically using image correlations, but in some cases such an automatic shift alignment does not lead to a good result and the manual alignment of the images needs to be applied. In most cases it is enough to apply manual alignment for all measured distances at rotation angle $\theta=0$ and use the obtained shifts as a starting point for automatic alignment of all remaining angles. It appears that images taken from periodically ordered structures such as a photonic crystal are surprisingly difficult to align since the shifts by an integer number of lattice parameters can not be distinguished by automatic alignment algorithms. Since our samples are very well ordered and periodic, manual shift alignment needs to be applied. Shift of the images varies a lot from sample to sample and from distance to distance and typical values of the shift are within $50-150 \mathrm{~nm}$ in all directions. After all images at the different distances for every angle are aligned with respect to each other, the phase retrieval can be launched. After the phase retrieval is finished the data consist of a stack of phase maps for every rotation angle. In order to obtain a 3D structure from the phase maps we need to apply a standard tomographic 3D reconstruction based on filtered back projection. 


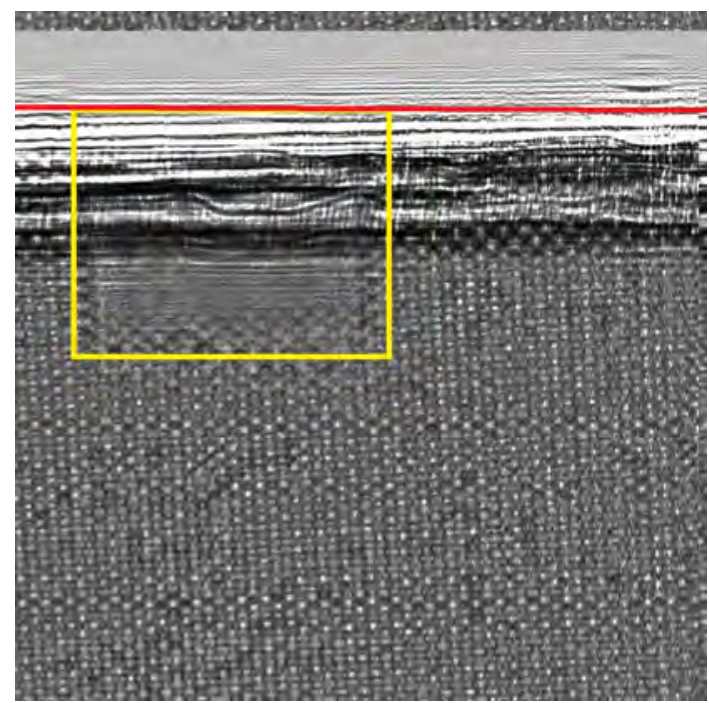

Figure 5.3: A typical transmission radiogram from $3 \mathrm{D}$ photonic crystal sample $\mathbf{A}$ measured with $20 \mathrm{~nm}$ resolution. The sample consists of a $2 \mathrm{D}$ photonic crystal structure and a 3D structure. The area of the 3D photonic crystal structure is indicated with the yellow rectangle. The straight red line indicates the approximate position of silicon to air interface. Black and white lines at the top of the image are the result of Fresnel diffraction on the edge of the sample. The periodic pattern visible in the lower portion originates from the $2 \mathrm{D}$ array of pores.

\subsection{Results and discussions}

\subsubsection{Choice of the phase retrieval algorithm}

For comparison of phase retrieval algorithms we used two different phase retrieval approaches for sample A: approach 1.a for homogeneous absorbing object and Paganin phase retrieval approach based on the transport of intensity equation. In the approach 1.a for homogeneous absorbing object the ratio between $\delta / \beta$ is used as a prior knowledge. For silicon at $17 \mathrm{keV}$ the ratio $\delta / \beta=174$ was obtained from the look up table [38]. A typical phase map out of the stack is shown in the figure 5.4 and the gray value cross section taken along the yellow line is shown in the figure $5.5(\mathrm{a})$. The gray value is proportional to the relative phase delay in the transmission and exhibits a linear behavior. The linear behavior of the phase delay is explained by the linear increase in sample thickness along the $X$ axis where the cross section was taken.

Sample A was also analyzed using the Paganin phase retrieval approach. The Paganin approach was used in the recursive way - the phase maps obtained after the first phase retrieval iteration are used as a preliminary information for the next iteration. The gray value cross section through the phase map is shown in Figure 5.5(b). The gray value that is proportional to the relative phase delay of the beam transmitted through the object exhibits a linear behavior which agrees 


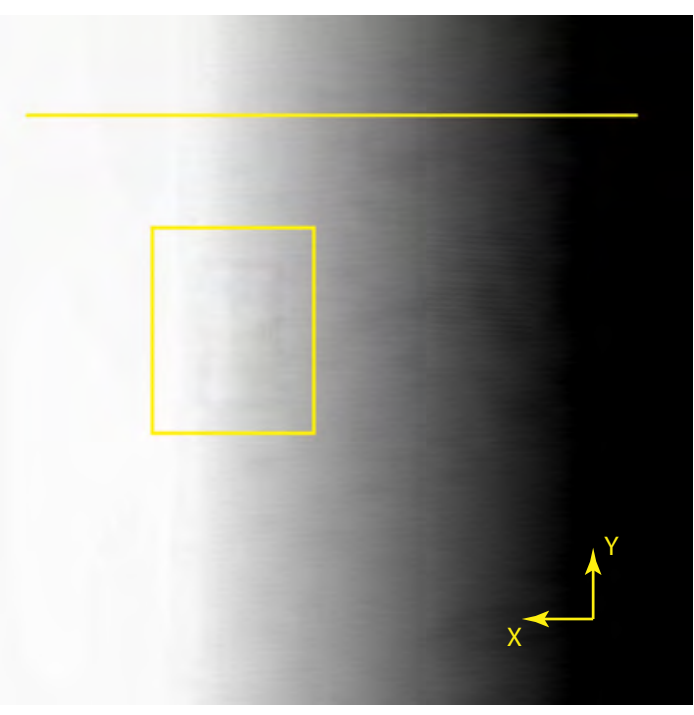

Figure 5.4: A typical phase map obtained for sample $\mathbf{A}$ at 0 degree rotation angle using phase retrieval approach 1.a. The gray scale represents relative phase delay of the transmitted beam with white color corresponding to zero relative phase delay. The $3 \mathrm{D}$ structure is located inside the yellow rectangle. The straight yellow line indicates where the gray value cross section was taken in Figure 5.5(a).

with the linear increase in sample thickness along the $X$ axis. It is noticed that there are less fluctuations in case of Paganin approach compared to approach 1.a. In order to make a quantitative comparison we obtain the maximum gray value contrast between air and silicon for both phase retrieval approaches. The gray value is proportional to the phase delay obtained after the x-ray the beam is propagated through the sample. The sample is a silicon slab of homogeneous thickness and thus the projected thickness of the sample is linear along the $X$ axis resulting in the linear phase delay. The fluctuations of the phase especially at the silicon to air interface indicate the presence of artifacts and thus will result in the distorted image after the reconstruction. It is seen from Figure 5.5 that both phase retrial approaches give smooth linear dependence of the phase delay but in case of Paganin phase retrieval approach there are less phase fluctuations of the phase near the Si to air interface. Therefore we conclude that the Paganin phase retrieval approach is more preferable for this kind of sample.

After obtaining the stacks of phase maps from the two approaches the tomographic reconstruction is applied to both of them. The output of the reconstruction process is a stack of $2 \mathrm{D}$ slices in plane with the beam propagation direction and with gray values proportional to the electron density of the material. A comparison of reconstructed result for two slices - from the middle of $3 \mathrm{D}$ structure (a) and from the area with only $2 \mathrm{D}$ structure (b) is shown in figure 5.6. The dark regions show high material density and the light regions show low material density. The $2 \mathrm{D}$ array of pores is indicated on the picture with the red rectan- 


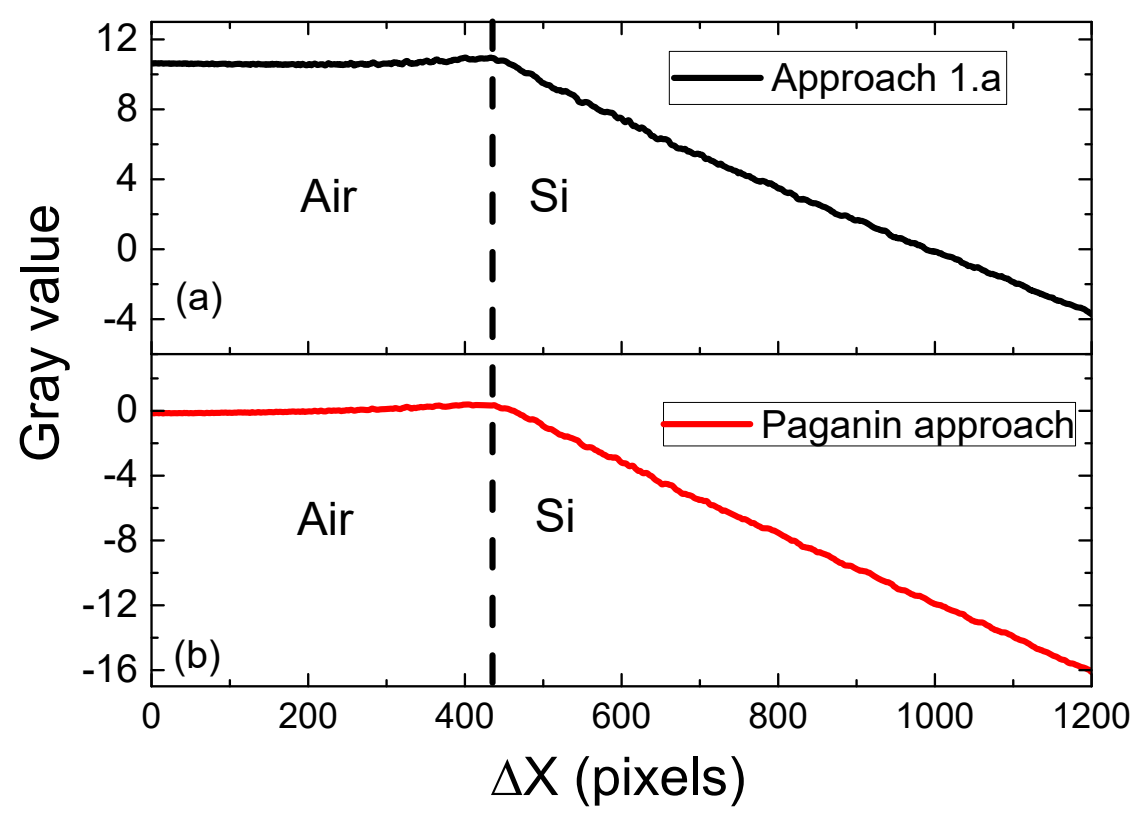

Figure 5.5: Gray value cross section taken from the retrieved phase maps of the sample A. The white color corresponds to the zero relative phase delay. Silicon to the air interface is located approximately at the distance 450 pixels. (a) The cross section from the phase map retrieved with approach 1.a for the homogeneous absorbing object. (b) The gray value cross section for the phase map retrieved using Paganin approach.

gle. The straight periodic lines in $2 \mathrm{D}$ region are the pores etched in the silicon, whereas the lines in air or in bulk silicon are measurement artifacts. The 3D structure is located within the yellow rectangle. The white area within the 3D structure gives an indication of the internal void inside the crystal. We associate the formation of a void in the structure to the capillary drying "stiction" after the infiltration of a quantum dot suspension for optical measurements and possible fabrication errors. Notably, the presence of the void inside the structure is invisible for the external inspections such as SEM imaging. There is a clear difference in the quality between images obtained with phase retrieval approach 1.a for the homogeneous absobtive object and Paganin approach based on the transport of intensity equation. Both in 3D and 2D areas the structure appears to be sharper in case of Paganin recursive phase retrieval. The air area contains no sample, therefore in the case of perfect reconstruction the material density should be constant and close to zero. Therefore we conclude that the distortions appearing in the air area are tomographic reconstruction artifacts. It is clear from the images that the number of artifacts is smaller in case of the Paganin 
recursive phase retrieval approach than in the case of 1.a approach. From this comparison we conclude that for our structures the Paganin approach reveals the better image quality and with less artifacts. In combination with the phase behaviour we dedcided to emply only Paganin approach from here on.
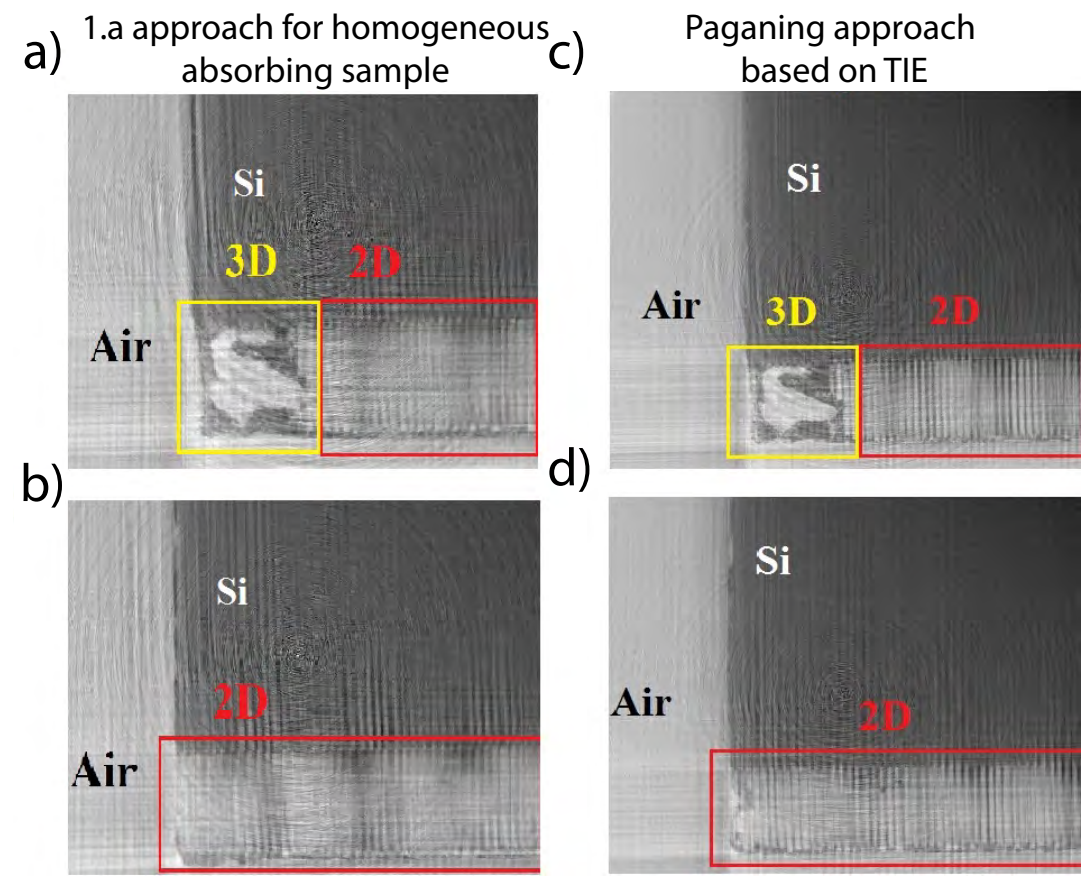

Figure 5.6: (a,b) Two slices of sample A reconstructed from the experiment using phase retrieval approach 1.a. (c,d) Two slices of sample $\mathbf{A}$ reconstructed with Paganin phase retrieval approach. Slices (a) and (c) are located in the middle of the 3D structure and slices (b) and (d) are located in the 2D region. Dark region is bulk silicon and light region is air. The $3 \mathrm{D}$ structure is indicated with yellow rectangle, the $2 \mathrm{D}$ array of pores is indicated with red rectangle. The straight periodic lines in the $2 \mathrm{D}$ region represent pores etched in the silicon. The lines outside $2 \mathrm{D}$ region in the bulk silicon are measurement artifacts. The white area within the 3D structure is an internal void inside the crystal due to stiction.

One of the common tomographic artifacts appears as circular rings centered around the rotation axis of the sample. Such rings are the results of beam imperfections that are independent on the sample rotation and thus do not move on the detector when the sample is rotating. There are two possible ways to overcome this distortion. A first one is a post processing of the image and computational correction for the rings. Another one is to adjust the experiment is such a way that a sample performs a small random move at every measured angle. Thus the relative position of distortions introduced by the beam shifts for each angle and therefore after tomographic reconstruction averages away. The comparison between the reconstructed image after the experiment with and without random 
displacement of the sample and is shown in Figure 5.7 (a) and (b). Image in the Figure 5.7 (a) has clear ring distortion artifacts centered at the top of the image causing the blurriness of the structure. In comparison the image in the Figure 5.7 (b) is clear from the circular distortions and thus the structure of interest has better contrast.

a)

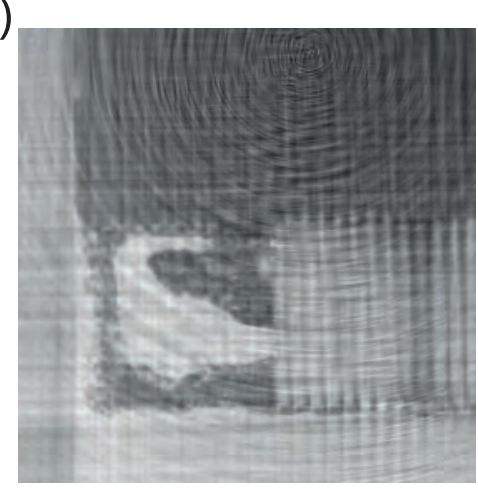

b)

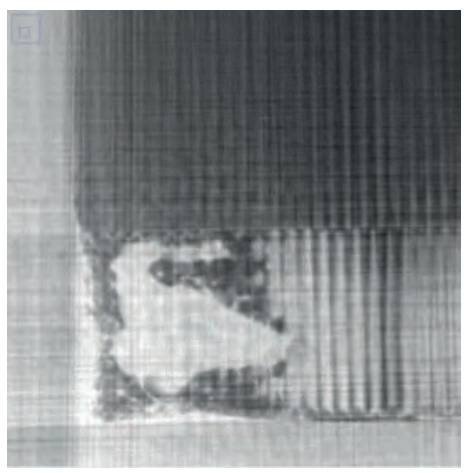

Figure 5.7: Comparison between the reconstructed image (a) without use of random displacement of the sample between scans and (b) with random displacement of the sample. It is clearly seen that circular distortions of the image vanish when the sample is randomly displaced.

\subsubsection{Choice of the resolution}

To compare the tomography results at different resolutions samples were studied in experiments with 20 and $10 \mathrm{~nm}$ resolution. Sample A reported above in a study with $20 \mathrm{~nm}$ resolution was also studied with the experiment with the ultimate $10 \mathrm{~nm}$ resolution. Based on the results of previous subsection 5.4.1, the Paganin recursive algorithm was chosen for the phase retrieval.

Figure 5.8 shows the comparison between the reconstructed slices of sample $\mathbf{A}$ with 10 and $20 \mathrm{~nm}$ resolutions. The lateral shift of the images is caused by the shift in the sample position in the chamber in between the two experiments. From Figure 5.8 it is seen that the image obtained with $10 \mathrm{~nm}$ resolution is sharper than the image obtained with $20 \mathrm{~nm}$ resolution. To characterize the contrast on both images we take a gray value cross-section through the reconstructed slices. To compare the data taken in different experiments and to estimate the resolution, we propose a model consisting of a binary density function for silicon $\rho_{S i}$ and vacuum $\rho_{v a c}$, convoluted with a Gaussian resolution function [39]. This model serve two purposes, one is to obtain Gaussian width $\sigma$ as an effective resolution of the image and second is to obtain the value of pore diameter and thickness of Si walls from the fit. Here we consider the Gaussian width $\sigma$ since we are interested in the choice of resolution and in the section 5.4.3 we will discuss the pore diameter and Si walls thicknesses obtained from the model. The density function assumes two values, namely $\rho_{S i}$ for silicon and $\rho_{v a c}$ for vacuum: 

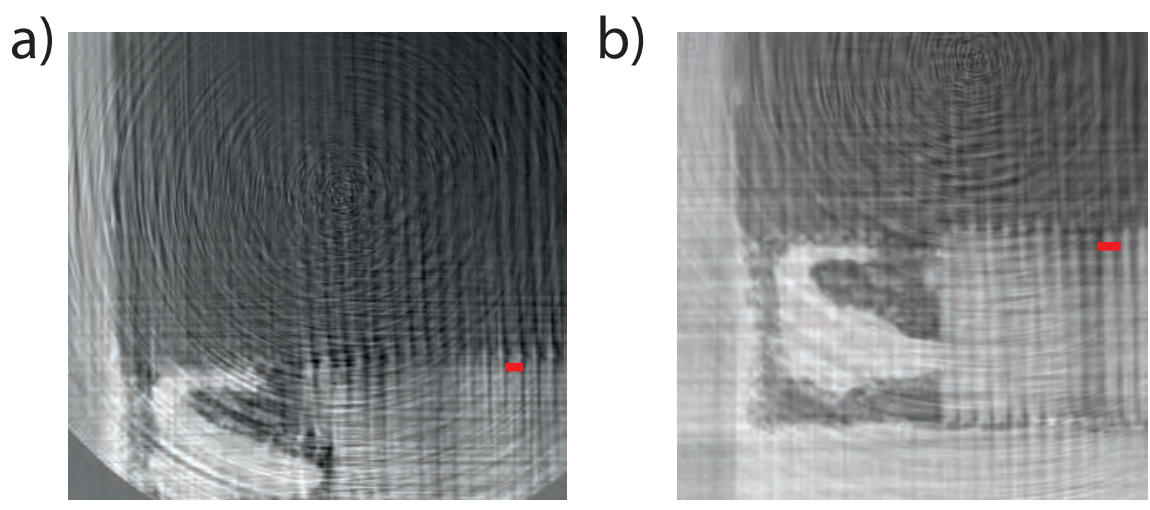

Figure 5.8: Comparison between the reconstructed slices from the data (a) obtained with $10 \mathrm{~nm}$ resolution and (b) with $20 \mathrm{~nm}$ resolution. Red lines indicate the positions where electron density profile was taken to model the data.

$$
\rho(x) \equiv \begin{cases}\rho_{v a c} & \text { if } x<-a / 2 \\ \rho_{S i} & \text { if }-a / 2<x<a / 2 \\ \rho_{v a c} & \text { if } x>a / 2 .\end{cases}
$$

The density $\rho(x)$ is convoluted with a normalized Gaussian resolution function with center $b$ and width $\sigma$ :

$$
\begin{array}{r}
\rho_{\text {mod }}(x)=\rho(x) * G(x)=\int_{-\infty}^{\infty} \rho(\tau) G(x-\tau) \mathrm{d} \tau= \\
\frac{\rho_{S i}}{2}\left(\operatorname{erfc}\left(\frac{b-a / 2-x}{\sqrt{2} \sigma}\right)-\operatorname{erfc}\left(\frac{b+a / 2-x}{\sqrt{2} \sigma}\right)\right)+\rho_{v a c}
\end{array}
$$

with $\operatorname{erfc}(x)$ the complementary error function. The density model $\rho_{\bmod }(x)$ is fitted to the experimental data. The adjustable parameters of the model are the width $a$ and center position $b$ of a silicon wall, the Gaussian width $\sigma$, the silicon gray scale $\rho_{S i}$, and the vacuum gray scale density $\rho_{v a c}$. Thus we can compare extracted $\sigma$ for images taken at different resolutionss. We take a cross section through the single pore at each slice as shown in Figure 5.8 with the red lines. The resulting cross section, convolution function and the model density are shown in Figure 5.9 (a) for the slice measured with $10 \mathrm{~nm}$ resolution and (b) for the slice measured with $20 \mathrm{~nm}$ resolution. The pore diameters and a Gaussian resolution widths for slices measured with different resolutions are the following: from the data obtained at $10 \mathrm{~nm}$ resolution the pore diameter is found to be $d_{10 \mathrm{~nm}}^{\text {model }}=392 \mathrm{~nm}$, the Gaussian resolution width $\sigma_{10 \mathrm{~nm}}=18 \mathrm{~nm}$. From data obtained with $20 \mathrm{~nm}$ resolution the pore diameter is $d_{20 \mathrm{~nm}}^{\text {model }}=380 \mathrm{~nm}$ with a Gaussian resolution width $\sigma_{20 \mathrm{~nm}}=41 \mathrm{~nm}$. The Gaussian width is nearly twice smaller in case of data measured with $10 \mathrm{~nm}$ resolution than the Gaussian width for the data measured with $20 \mathrm{~nm}$ resolution which agrees with the experimental resolution setting. The amplitude of the gray value contrast between the silicon 


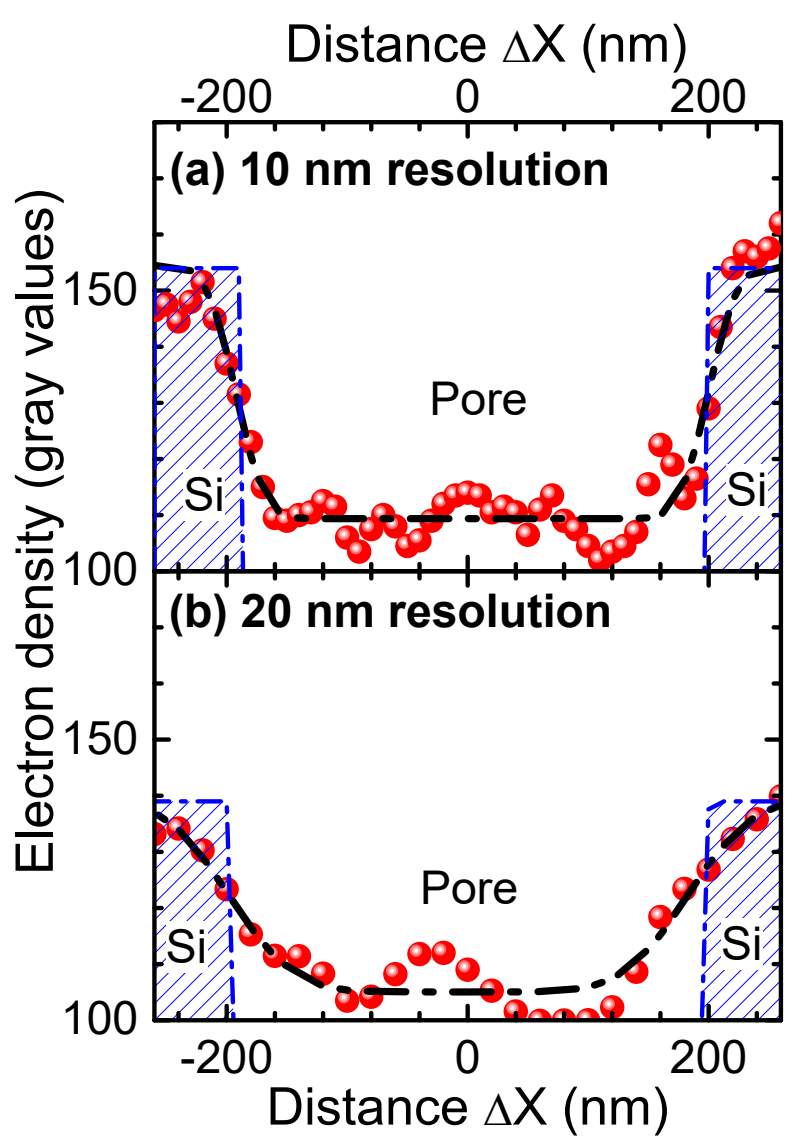

Figure 5.9: Electron density profile through the single pore for the data (a) measured with $10 \mathrm{~nm}$ resolution and (b) with $20 \mathrm{~nm}$ resolution. The position where the electron density profiles were taken are shown in Figure 5.8 with red lines. The black dashed curve is the convolution of a square density function (blue hatched bars) and a Gaussian resolution function.

and air is clearly larger in case of data obtained $10 \mathrm{~nm}$ resolution (Figure 5.9 (a)) than in case of $20 \mathrm{~nm}$ resolution (Figure 5.9 (b)). With $10 \mathrm{~nm}$ resolution the amplitude contrast between the $\mathrm{Si}$ and air obtained from the fit model is $\Delta \rho_{10 \mathrm{~nm}}=45$ and with $20 \mathrm{~nm}$ resolution data the contrast is $\Delta \rho_{10 \mathrm{~nm}}=34$. We associate the larger contrast of the gray value in case smaller resolution with the smaller voxel size and thus the electron densities are averaged over a smaller volume. This results in a sharper image in case of $10 \mathrm{~nm}$ resolution experiment as is clearly seen in Figure 5.8. On the other hand, a $10 \mathrm{~nm}$ resolution can sometimes not be employed when the field of view (equal to a number of pixels times the set resolution) is too small for a sample under study. 
After comparing multiple phase retrieval approaches and different experimental procedures we conclude that for our structures the best way of performing x-ray tomography is with $10 \mathrm{~nm}$ resolution using the random sample displacement to remove artifacts and applying Paganin recursive phase retrieval approach to the obtained data.

\subsubsection{Results and discussion on photonic crystals}

Combining all 2D slices of the electron density distribution, we obtain a full $3 \mathrm{D}$ real space representation of the structure. The list of samples with their internal lab names and fabrication history is in the Appendix C. The full 3D representation of the sample $\mathbf{A}$ measured with $10 \mathrm{~nm}$ resolution and retrieved with Paganin phase retrieval approach is shown in Figure 5.10.

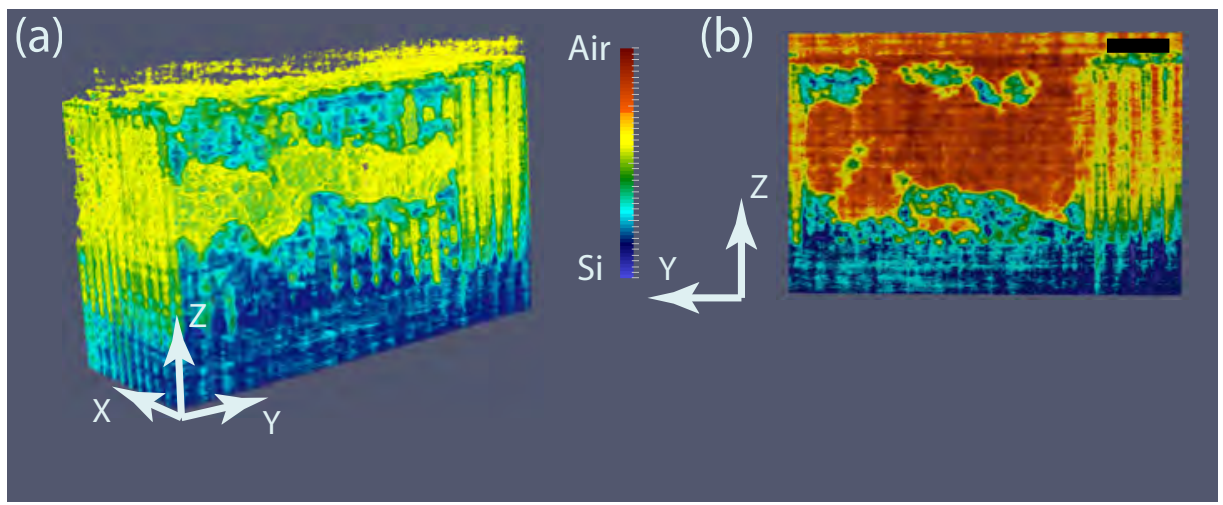

Figure 5.10: (a) Volume rendering of the sample A. The air is shown transparent for the clarity. The internal void is seen inside the structure in $Z Y$ plane. The void is attributed to the capillary forces while sample was drying after being kept in the quantum dots suspension. (b) Cross section in the $Y Z$ plane through the middle of the reconstructed volume. The internal void is clearly seen in the center of the slice. 2 microns scale bar is shown on the picture.

The internal void hidden from the outside observer is clearly seen inside the structure of the sample after tomographic reconstruction. The void formation is attributed (1) to the fabrication error resulting in the larger pore radius than designed and thus thinner silicon walls between pores and (2) capillary forces appearing after the sample was dried multiple times from a quantum dot suspension for spontaneous emission measurements. Since the 3D structure in this sample appeared to be broken the analysis was performed on the remaining surrounding $2 \mathrm{D}$ array of poress. The pore radius obtained from the tomography data is $r_{A}=190 \pm 20 \mathrm{~nm}$ and the pore depth is $d_{A}=6080 \pm 20 \mathrm{~nm}$. Comparing the pore radius to the design $r_{\text {design }}=163 \mathrm{~nm}$ it is clear that the errors were made during fabrication process resulting in wider pores than designed. The tapering of the pores was analyzed by measuring the pore radius at the different depths: at the 
top, in the middle and at the bottom. The pore radius at the top was found to be $r_{A}^{t o p}=190 \pm 20 \mathrm{~nm}$, at the middle it increased to $r_{A}^{m i d d l e}=200 \pm 20 \mathrm{~nm}$ and at the bottom the radius decreased to $r_{A}^{b o t t o m}=160 \pm 20 \mathrm{~nm}$. The summary of the characteristic values for each sample is given in table 5.4.3 below.

Sample $\mathbf{B}$ was measured with $20 \mathrm{~nm}$ resolution and reconstructed using the $\mathrm{Pa}-$ ganin recursive phase retrieval approach. A bird's-eye view of the reconstructed $3 \mathrm{D}$ structure is shown in Figure 5.11(a). This sample reveals a 3D structure inside and is thus analyzed in more details. The reconstructed sample volume is a cube that includes the $3 \mathrm{D}$ photonic crystal structure that is surrounded by bulk silicon below, and by the $2 \mathrm{D}$ array of deep pores. To keep the rich set of tomographic data tractable, we choose for analysis three $2 \mathrm{D}$ slices in arbitrary planes in the reconstructed volume, as shown in Figure 5.11(b). The slices are chosen in the $Z Y, Z X$ and $X Y$ symmetry planes of the photonic crystal structure. For simplicity, we first discuss the simple $2 \mathrm{D}$ array of pores that is shown in the $X Y$ slice in Fig. 5.11(c). The array reveals the designed centered rectangular structure. Several pores the top of the image are connected by pores running in the $X$-direction that are made in a second etch-step to obtain the $3 \mathrm{D}$ structure.

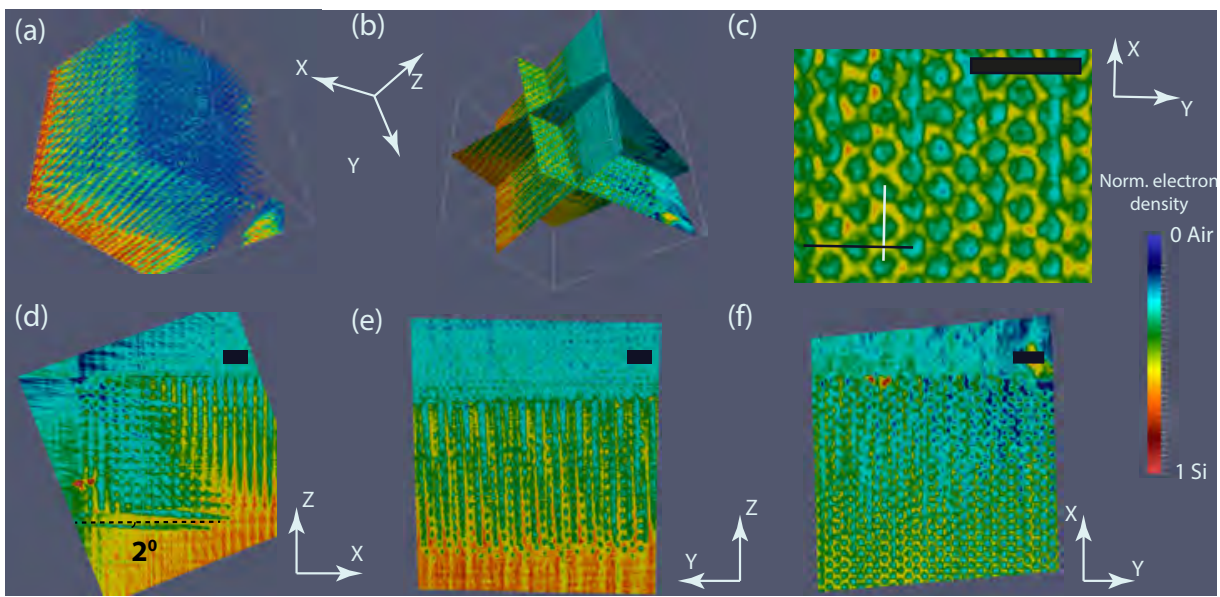

Figure 5.11: $3 \mathrm{D}$ rendered images of a $3 \mathrm{D}$ photonic band gap crystal sample B. $X, Y, Z$ axes are shown with each panel. a) Bird's-eye view of the reconstructed sample volume. The color scale is the normalized electron density interpolated between silicon $\left(\rho_{n}=\right.$ 1) and air $\left(\rho_{n}=0\right)$. For clarity, electron densities close to air are transparent. b) Locations of the 2D slices of the electron density chosen for detailed investigation. The reconstructed volume is shown as the outline and the view point is the same as in (a). c) Zoom-in of a 2D $X Y$ slice located at a depth $Z=400 \mathrm{~nm}$ below the crystal surface. Gray and black lines show trajectories where the gray value profiles were taken (Fig. 5.12). (d) $2 \mathrm{D}$ slice in the $Z X$ plane. Black dashed line indicated a $2^{\circ}$ misalignment of the pores. (e), (f) $2 \mathrm{D}$ slices of electron densities located in $Z Y$ and $X Y$ planes respectively. A 1 micron scale bar is shown on each slice. 


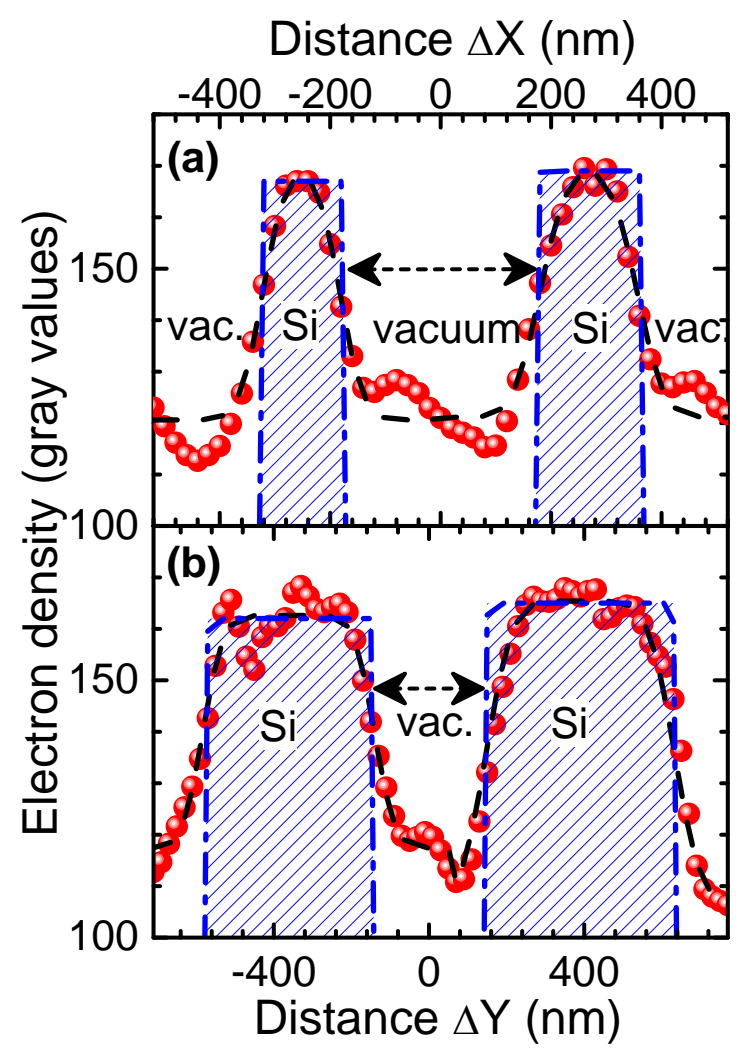

Figure 5.12: (a) Density profile in the $X$-direction, and (b) in the $Y$-direction through pores shown in Figure 5.11(c) (red circles). The black dashed curves are the convolution of a square density function (blue hatched bars) and a Gaussian resolution function.

We now discuss one-dimensional cross-sections through the 2D pore array along lines shown in Fig. 5.11(c) for a detailed comparison between the tomographic data and the structural design. Fig. 5.12(a) shows a cross-section in the $X$ direction, starting from a pore via a $\mathrm{Si}$ wall to a second pore and a second Si-wall into a third pore. The distance between the centers of the Si walls is $\Delta X=520 \pm 20 \mathrm{~nm}$. Fig. 5.12(b) shows a cross-section in the $Y$-direction, also from a pore via a Si-wall, a second pore and a second $\mathrm{Si}$-wall to a third pore. The distance between the centers of the Si walls is $\Delta Y=750 \pm 20 \mathrm{~nm}$. Importantly, the ratio $\frac{\Delta X}{\Delta Y}=1.44 \pm 0.06$ agrees with the designed $\sqrt{2}$ ratio required for a cubic structure. Analyzing the neares neighbor distance in the $X$-direction over 14 unit cells (see Figure 5.13) yields a statistical mean $\Delta X_{m}=481 \pm 20 \mathrm{~nm}$ with a standard deviation $\sigma_{m}=30 \mathrm{~nm}$, which agrees excellently with the designed lattice parameter $c=488 \mathrm{~nm}$. The obtained mean value is shown in the Figure 5.13 with black dashed line, standard deviation is shown with the gray bar and the design lattice parameter is shown with red solid line. In the $Y$-direction, we find 


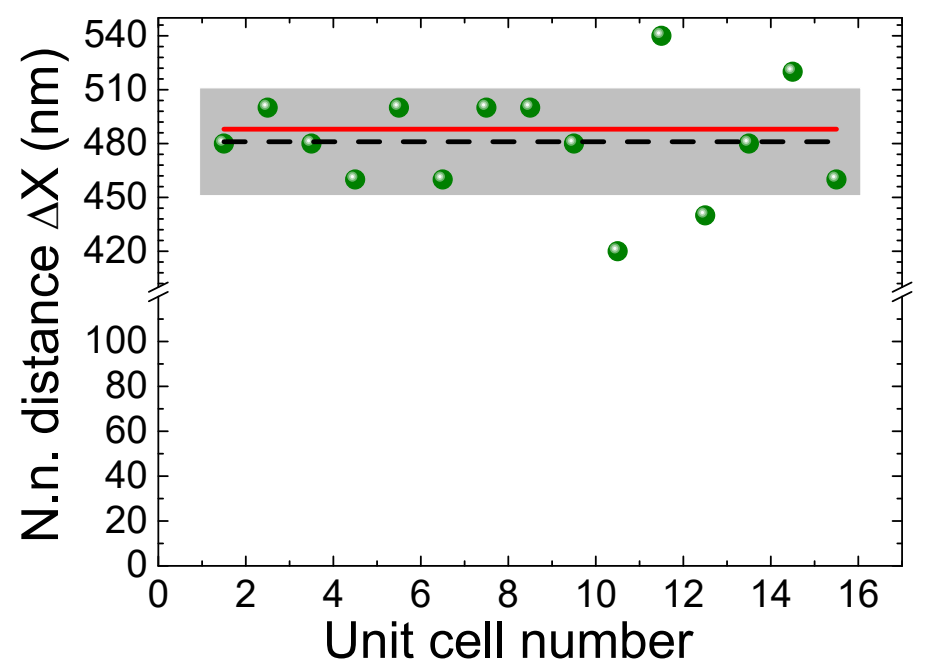

Figure 5.13: Nearest neighbor distance between pores in a 3D photonic crystal sample B measured from tomography data over 15 unit cells. The distance between pore in the $1^{\text {st }}$ unit cell and it the $2^{\text {nd }}$ unit cell is shown number 1.5. Mean value $\Delta X_{m}=$ $481 \pm 20 \mathrm{~nm}$ is shown with black dashed line and standard deviation $\sigma_{m}=30 \mathrm{~nm}$ is shown with the gray bar. The mean value agrees well with the designed lattice parameter $c=488 \mathrm{~nm}$ shown with red solid line.

$\Delta Y_{m}=700 \mathrm{~nm}$ with $\sigma_{m}=27 \mathrm{~nm}$ standard deviation, in very good agreement with the designed lattice parameter $a=686 \mathrm{~nm}$. The results confirm that the structure is periodic over many unit cells, with average lattice parameters that match the designed ones very well.

The data in Fig. 5.12(a) are modeled as described in a section 5.4.2 above with a binary model consisting of two Si walls and air in between them. From the fit model a width of one wall was found to be $w_{1}=140 \mathrm{~nm}$ with a Gaussian resolution width $\sigma_{1}=32 \mathrm{~nm}$, and the second wall has $w_{2}=176 \mathrm{~nm}$ width with $\sigma_{2}=37 \mathrm{~nm}$. Given the voxel size of $20 \mathrm{~nm}$, the widths of the Si walls agree within the resolution with the designed width $w=156 \mathrm{~nm}$, thus the structural design was faithfully realized. The corresponding pore diameter of $340 \mathrm{~nm}$ obtained from the cross section agrees well with the designed diameter of $328 \mathrm{~nm}$. The model for $Y$-cross-sections shown in Fig. 5.12(b) consist of two Si walls with $w_{1}=441 \mathrm{~nm}$, with a Gaussian resolution width $\sigma_{1}=41 \mathrm{~nm}$ and $w_{2}=484 \mathrm{~nm}$ with $\sigma_{2}=52 \mathrm{~nm}$. The widths of the $\mathrm{Si}$ walls are somewhat wider than the designed width of $358 \mathrm{~nm}$, possibly due to a slight deformation of the pore. The Gaussian resolution of $30 \mathrm{~nm}$ in the $X$-direction (45 $\mathrm{nm}$ in the $Y$-direction) agrees well with the focus size of $20 \mathrm{~nm}$ (30 nm in the $Y$-direction); it is reasonable that the modeled resolution is somewhat larger in view of the fact that it is the result of a tomographic reconstruction where several data analysis steps described above were taken. 
Figure 5.11(d) shows a $Z X$ slice that contains both arrays of pores and allows us to determine the maximum depths of both sets of pores. The $Z$-pores have a depth of $6280 \pm 20 \mathrm{~nm}$ and a diameter of $366 \pm 20 \mathrm{~nm}$ at the middle of the depth, and a correspondingly high aspect ratio of $17.20 \pm 0.04$, as expected from the deep reactive-ion etching settings [40]. Previously, the aspect ratio of pores deeply etched in silicon could only be assessed ex-situ and destructively, by SEM inspection of ion-milled slices or of a cleaved structure [36, 40]. The $Z$-pore diameter at the surface is found to be $322 \pm 20 \mathrm{~nm}$ and is reduced to $300 \pm 20 \mathrm{~nm}$ at the bottom due to the tapering. The $X$-pores have an even greater depth of $8000 \pm 20 \mathrm{~nm}$, corresponding to a high aspect ratio of $24.3 \pm 0.04$. This is the first unequivocal observation that a second set of deep-etched pores runs even deeper than a first set. This effect can be explained as follows: one limiting factor in the deep etching of pores is the diffusion of reaction products that cannot easily diffuse out of a deep and narrow pore. This limitation affects the first set of pores, but not the second set of pores: While the second set of pores is being etched, the reaction products diffuse outwards through the first set of pores. The $X$-pores pore diameter thus also differs from $Z$-pores diameter and is found to be $272 \pm 20 \mathrm{~nm}$ at the top surface, $328 \pm 20 \mathrm{~nm}$ in the middle of the pore depth and $200 \pm 20 \mathrm{~nm}$ at the bottom.

A salient feature in Figure 5.11(d) is that the pores running in the $X$-direction are not exactly perpendicular to the pores in the $Z$-direction. It appears that the angle between the pore arrays systematically deviates from the $90^{\circ}$ design by $2^{\circ}$; from results on several crystals we find at most $6^{\circ}$. This means that the crystal structure is slightly monoclinic. Nevertheless, plane-wave band structure calculations reveal that the $3 \mathrm{D}$ photonic band gap is robust to a $6^{\circ}$ shear of the cubic crystal structure [8]: the gap hardly changes from $24 \%$ relative bandwidth to more than $21 \%$, plenty for strong spontaneous emission control and other metamaterial applications.

Figure 5.11(e) shows an $Z X$ slice that crosses the first set of pores along their greatest depth. All pores in the slice have a similar etched depth of $6000 \pm$ $20 \mathrm{~nm}$ confirming that the deep reactive etching process gives a homogeneous pore formation in bulk Si, including a substantial etch depth. Figure 5.11(f) shows an $X Y$ slice that contains the second array of pores. It is seen that the depth of the pores varies between $5000 \pm 20$ and $8500 \pm 20 \mathrm{~nm}$ at different $Y$. We attribute this pore depth variation to changes in etching process due to penetration of the etchant and the etching products through the array of pores that was first etched. As a consequence, optical transport measurements will reveal smooth spectra, without Fabry-Perot fringes. The main characteristics of the sample measured from tomography data are listed in Table 5.4.3 at the end of the section.

The next 3D photonic crystal sample under investigation is sample $\mathbf{C}$ that was fabricated using a novel single step etch mask approach described in details in chapter 2. This approach improves the alignment of the etch mask during fabrication process and allows to achieve less structural deviations from the design. Figure 5.14(a) shows a volume rendering of the3D photonic crystal sample. Electron densities close the air are shown transparent for clarity. Two sets of pores 


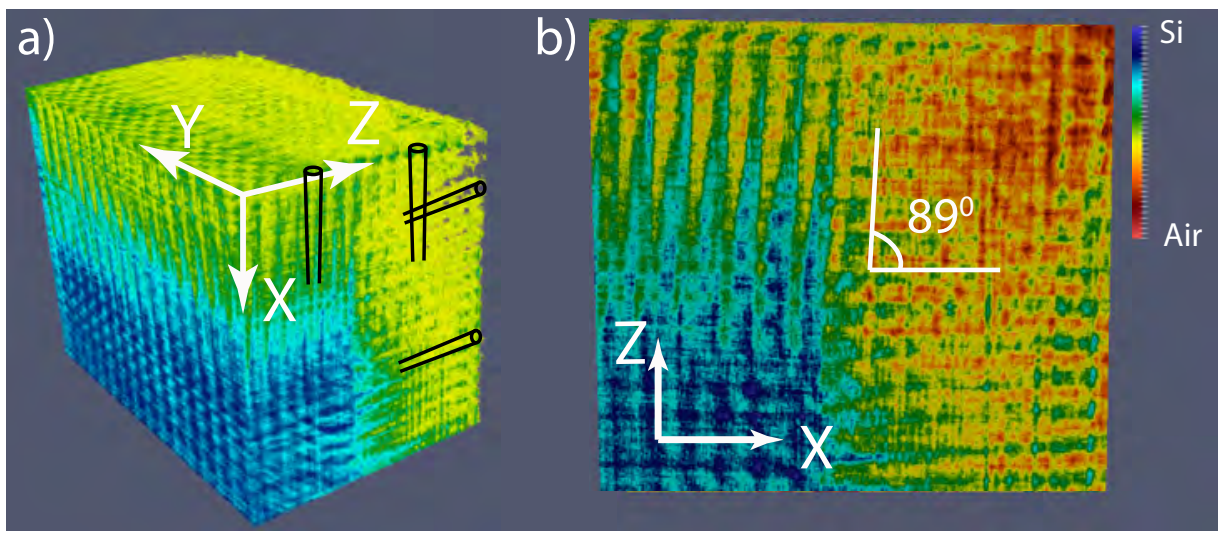

Figure 5.14: (a) Volume rendering of the $3 \mathrm{D}$ photonic crystal sample $\mathbf{C}$. Two sets of pores are running in $X$ and $Z$ direction. Black lines are the guides for the eye indicating the positions of the pores in the crystal. (b) Cross section in $X Z$ plane through the reconstructed volume. Two sets of pores in $X$ and $Z$ direction are seen to be crossing each other under 89 degree angle.

are running in $X$ and $Z$ direction. Figure 5.14(b) shows the cross section in $X Z$ plane. The pore diameter for the pores running in $Z$ direction which is the first etch side is found to be $370 \pm 10 \mathrm{~nm}$ at the top, $410 \pm 10 \mathrm{~nm}$ at the middle depth and $350 \pm 10 \mathrm{~nm}$ at the bottom. Pores exhibit common trend to get wider in the middle and reduce in diameter at the bottom known, as tapering effect. The depth of the pores in $Z$ direction is $3770 \pm 10 \mathrm{~nm}$. Pores in $X$ direction, the second etch direction differ from the first direction and have $300 \pm 10 \mathrm{~nm}$ diameter at the top, $310 \pm 10 \mathrm{~nm}$ diameter at the middle and $155 \pm 10 \mathrm{~nm}$ at the bottom. Thus pores etched on the second side exhibit larger tapering and overall smaller radius than pores that were etched first. This is explained with residuals of the protective layer left from the Bosch etching process on the mask for the second etch side. The depth of the pores in $X$ direction is $4630 \pm 10 \mathrm{~nm}$. In this sample two sets of pores are seen to be crossing each other under $89 \pm 1$ degree angle. All the characteristics of the sample measured from tomography data are listed in Table 5.4.3 at the end of the section. The angular angular alignment of pores is thus significantly improved to be within the error bar compared to observed above misalignment of 2 degrees in the sample B. Thus tomography data shows improvement in the fabrication process of the photonic crystal. Additionally one more sample $\mathbf{D}$ was studied in tomography experiments. On the sample $\mathbf{D}$ two photonic crystal structures were investigated - Structure $D_{1}$ and Structure $D_{2}$ that are 3D inverse woodpile photonic crystals without embedded defects intended for reflectivity study described in Chapter 6. From tomography pores in the structure $D_{1}$ have the depth $l_{Z}=4580 \pm 10 \mathrm{~nm}$ in $Z$ direction and $l_{X}=2940 \pm 10 \mathrm{~nm}$ in $X$ direction. As listed in Table 5.4.3 dimeter of the pores in $Z$ direction on the top surface is found to be $d_{z}^{\text {top }}=304 \pm 10 \mathrm{~nm}$, in the 
middle of the pore $d_{z}^{\text {mid }}=318 \pm 10 \mathrm{~nm}$ and at the bottom $d_{z}^{\text {bot }}=300 \pm 10 \mathrm{~nm}$. For pores in $X$ direction the diameters are found to be $d_{x}^{\text {top }}=270 \pm 10 \mathrm{~nm}$, $d_{x}^{\text {mid }}=246 \pm 10 \mathrm{~nm}$ and $d_{x}^{b o t}=160 \pm 10 \mathrm{~nm}$. For the Structure $D_{2}$ in $Z$ direction the depth of the pores is $l_{Z}=3440 \mathrm{~nm}$ and in $X$ direction $l_{X}=2800 \pm 10 \mathrm{~nm}$. The $Z$-pores diameter at the top is found to be $d_{z}^{\text {top }}=301 \pm 10 \mathrm{~nm}$, at the middle depth $d_{z}^{\text {mid }}=280 \pm 10 \mathrm{~nm}$ and at the bottom $d_{z}^{\text {bot }}=280 \pm 10 \mathrm{~nm}$. For the pores running in $X$ direction $d_{x}^{t o p}=300 \pm 10 \mathrm{~nm}, d_{x}^{\text {mid }}=266 \pm 10 \mathrm{~nm}$ and $d_{x}^{b o t}=222 \pm 10 \mathrm{~nm}$. Tomography data are obtained for the Structures $D_{1}$ and $D_{2}$ are used in order to extract an important for optical properties averaged over structure ratio of $R / a$ where $R$ is the pore radius and $a$ is the lattice spacing. To do so we obtain the electron density of the air $\rho_{\text {air }}$ by averaging the gray value in the region outside the crystal and silicon bar, the density of silicon $r h o_{s i}$ by averaging the gray value in the bulk silicon regions and the average density of 3D structure $\rho_{3 D}$ by averaging the gray value over the entire volume of $3 \mathrm{D}$ structure. Considering the following relations:

$$
\begin{aligned}
& \rho_{S i} \cdot v_{S i}+\rho_{a i r} \cdot v_{a i r}=\rho_{3 D} \\
& v_{S i}+v_{a i r}=1
\end{aligned}
$$

where $v_{S i}$ is the volume fraction of $\mathrm{Si}$ in the structure and $v_{\text {air }}$ is the volume fraction of air in the structure, we can find the volume fraction of air in the structure that is directly related to the $R / a$ ratio as is shown in Figure 1.3 in the Chapter 1 . The obtained from tomography data ratio of $R / a$ we call $R / a_{\text {tomo }}$ and in the later chapter compare to the $R / a$ ratio extracted from reflectivity measurements. For the Structure $D_{1}$ the $R / a$ ratio obtained from tomography data is $R / a_{\text {tomo }}=0.197 \pm 0.010$ and for the Structure $D_{2} R / a_{\text {tomo }}=0.21 \pm$ 0.015. The good correspondence of the obtained ratios to the optical reflectivity measurements is discussed in Chapter 6 .

Below we provide a table summarizing the characteristic values of pore diameter, depth and angular misalignment for all samples discussed above.

\subsection{Summary}

In this chapter we describe recently developed technique zoom in nanotomography which allows to perform 3D imaging of the material with nm resolution. We are the first to apply zoom in nanotomography with ultimate 10 and 20 $\mathrm{nm}$ resolution to the fabricated $3 \mathrm{D}$ photonic nanostructures. We provide experimental scheme as well as detailed theoretical description of the required phase retrieval algorithms for zoom in tomography. We use our experimental data of tomographic scans measured on 3D silicon photonic crystal samples to compare different phase retrieval algorithms and different experimental approaches for Xray nanotomography. After comparing multiple phase retrieval approaches and different experimental procedures we conclude that for our structures the optimal way of performing X-ray tomography is with $10 \mathrm{~nm}$ resolution using additional the random displacement of the sample in order to remove artifacts. In the process of data analysis due to periodic geometry of our structures it appeared to 


\begin{tabular}{|c|c|c|c|}
\hline Sample & $\begin{array}{l}\text { Pore diameter } d \text { in } \\
Z \text { and } X \text { directions }\end{array}$ & $\begin{array}{l}\text { Pore depth } l \text { in } \\
Z \text { and } X \text { direction }\end{array}$ & $\begin{array}{l}\text { Angle } \\
\text { between sets } \\
\text { of pores }\end{array}$ \\
\hline $\mathbf{A}$ & $\begin{array}{l}d_{z}^{t o p}=380 \pm 20 \mathrm{~nm} \\
d_{z}^{m i d}=400 \pm 20 \mathrm{~nm} \\
d_{z}^{b o t}=160 \pm 20 \mathrm{~nm}\end{array}$ & $l_{z}=6080 \pm 20 \mathrm{~nm}$ & $\begin{array}{l}\text { Not possible } \\
\text { to determine } \\
\text { since the } \\
\text { structure is } \\
\text { destroyed }\end{array}$ \\
\hline \multirow[t]{2}{*}{$\bar{B}$} & $\begin{array}{l}d_{z}^{t o p}=322 \pm 20 \mathrm{~nm} \\
d_{z}^{m i d}=366 \pm 20 \mathrm{~nm} \\
d_{z}^{b o t}=300 \pm 20 \mathrm{~nm}\end{array}$ & $l_{z}=6280 \pm 20 \mathrm{~nm}$ & $88 \pm 1^{o}$ \\
\hline & $\begin{array}{l}d_{x}^{t o p}=272 \pm 20 \mathrm{~nm} \\
d_{x}^{m i d}=328 \pm 20 \mathrm{~nm} \\
d_{x}^{b o t}=200 \pm 20 \mathrm{~nm}\end{array}$ & $l_{x}=8000 \pm 20 \mathrm{~nm}$ & \\
\hline \multirow[t]{2}{*}{$\mathbf{C}$} & $\begin{array}{l}d_{z}^{t o p}=370 \pm 10 \mathrm{~nm} \\
d_{z}^{\text {mid }}=410 \pm 10 \mathrm{~nm} \\
d_{z}^{b o t}=350 \pm 10 \mathrm{~nm}\end{array}$ & $l_{z}=3770 \pm 10 \mathrm{~nm}$ & $89 \pm 1^{\circ}$ \\
\hline & $\begin{array}{l}d_{x}^{t o p}=300 \pm 10 \mathrm{~nm} \\
d_{x}^{m i d}=310 \pm 10 \mathrm{~nm} \\
d_{x}^{b o t}=155 \pm 10 \mathrm{~nm}\end{array}$ & $l_{x}=4630 \pm 20 \mathrm{~nm}$ & \\
\hline \multirow[t]{2}{*}{$D_{1}$} & $\begin{array}{l}d_{z}^{t o p}=304 \pm 10 \mathrm{~nm} \\
d_{z}^{m i d}=318 \pm 10 \mathrm{~nm} \\
d_{z}^{b o t}=300 \pm 10 \mathrm{~nm}\end{array}$ & $l_{z}=4580 \pm 10 \mathrm{~nm}$ & $89 \pm 1^{o}$ \\
\hline & $\begin{array}{l}d_{x}^{t o p}=270 \pm 10 \mathrm{~nm} \\
d_{x}^{m i d}=246 \pm 10 \mathrm{~nm} \\
d_{x}^{b o t}=160 \pm 10 \mathrm{~nm}\end{array}$ & $l_{x}=2940 \pm 10 \mathrm{~nm}$ & $89 \pm 1^{o}$ \\
\hline \multirow[t]{2}{*}{$D_{2}$} & $\begin{array}{l}d_{z}^{t o p}=301 \pm 10 \mathrm{~nm} \\
d_{z}^{m i d}=280 \pm 10 \mathrm{~nm} \\
d_{z}^{b o t}=280 \pm 10 \mathrm{~nm}\end{array}$ & $l_{z}=3440 \pm 10 \mathrm{~nm}$ & $89 \pm 1^{\circ}$ \\
\hline & $\begin{array}{l}d_{x}^{t o p}=300 \pm 10 \mathrm{~nm} \\
d_{x}^{m i d}=266 \pm 10 \mathrm{~nm} \\
d_{x}^{b o t}=222 \pm 10 \mathrm{~nm}\end{array}$ & $l_{x}=2800 \pm 10 \mathrm{~nm}$ & $89 \pm 1^{\circ}$ \\
\hline
\end{tabular}

Table 5.1: Main characteristics of the samples measured in X-ray tomography experiments. The pore diameters are listed separately for pores in $Z$ direction $d_{z}$ and $X$ direction $d_{x}$. $Z$ direction is the first etch direction and $Y$ direction is the second etch direction. Diameters are measured for each direction at the top of the pore close to the surface $d^{\text {top }}$, at the middle $d^{\text {mid }}$ and at the bottom $d^{\text {bot }}$. 
be necessary to perform manual shift alignment of projections taken at different distances. The best phase retrieval results are achieved using recursive Paganin approach.

We have studied a number of 3D photonic band gap crystals made from silicon by CMOS-compatible methods. We have managed to obtain the $3 \mathrm{D}$ material density throughout the fabricated crystals. We observed that the structural design is in most aspects faithfully realized by the fabricated nanostructure, apart from a shear of the cubic crystal structure for the sample fabricated with the two-step etch mask. We observed an improvement in structure alignment after applying a single step etch mask fabrication approach. We suggested a binary model that describe tomography data and allows to use them as an input for $a b$ initio numerical models in order to compute optical properties free from assumptions. We conclude that 3D X-ray tomography is a powerful tool for the structural characterization of any complex photonic nanostructure with arbitrary short- or long-range order with no need for irreversible sample preparation. Therefore, X-ray tomography has great potential to solve a wide variety of outstanding questions in metamaterial research and applications. 


\section{Bibliography}

[1] T. Ergin, N. Stenger, P. Brenner, J.B. Pendry, and M. Wegener, Threedimensional invisibility cloak at optical wavelengths, Science 328, 337-339 (2010) 77

[2] C.M. Soukoulis and M. Wegener, Past achievements and future challenges in the development of three-dimensional photonic metamaterials, Nature Photon, 5, 523 (2011) 77

[3] J.K. Gansel, M. Thiel, M.S. Rill, M. Decker, K. Bade, V. Saile, G. von Freymann, S. Linden, and M. Wegener, Gold helix Photonic metamaterial as broadband circular polarizer, Science 18, 1513-1515 (2009) 77

[4] J.E.G.J. Wijnhoven and W.L. Vos, Preparation of photonic crystals made of air spheres in titania, Science 281, 802-804 (1998) 77

[5] S. Noda, K. Tomoda, N. Yamamoto, and A. Chutinan, Full three-dimensional photonic bandgap crystals at near-infrared wavelengths, Science 289, 604-606 (2000) 77

[6] J.D. Joannopoulos, S.G. Johnson, J.N. Winn, and R.D. Meade, Photonic crystals, Molding the flow of light (Princeton University Press, Princeton NJ, 2008) $2^{\text {nd }}$ Ed. 77

[7] A. Chutinan and S. Noda, Effects of structural fluctuations on the photonic bandgap during fabrication of a photonic crystal, J. Opt. Soc. Am. B 16, 240244 (1999). 77

[8] L.A. Woldering, A.P. Mosk, R.W. Tjerkstra, and W.L. Vos, The influence of fabrication deviations on the photonic band gap of three-dimensional inverse woodpile nanostructures, J. Appl. Phys. 105, 093108: 1-10 (2009). 77, 100

[9] S. Hughes, L. Ramunno, J. F. Young, and J. E. Sipe, Extrinsic Optical Scattering Loss in Photonic Crystal Waveguides: Role of Fabrication Disorder and Photon Group Velocity, Phys. Rev. Lett. 94, 033903: 1-4 (2005) 77

[10] A.F. Koenderink, A. Lagendijk, and W.L. Vos, Optical extinction due to intrinsic structural variations of photonic crystals, Phys. Rev. B 72, 53102: 1-4 (2005). 77

[11] D. McMullan Scanning electron microscopy Science 17, 175-185 (1995) 77

[12] G. Binnig and H. Rohrer Scanning tunneling microscopy from birth to adolescence Rev. Mod. Phys. 59, 615-625 (1987) 77

[13] J. Goldstein, D.E. Newbury, D.C. Joy, C.E. Lyman, P. Echlin, E. Lifshim, L. Sawyer, and J.R. Michael, Scanning electron microscopy and X-ray microanalysis, (Springer, New York, 2003) 3rd Ed. 77

[14] R. Xu, C.-C. Chen, L. Wu, M. C. Scott, W. Theis, C. Ophus, M. Bartels, Y. Yang, H. Ramezani-Dakhel, M. R. Sawaya, H. Heinz, L. D. Marks, P. Ercius, 
and J. Miao Three-dimensional coordinates of individual atoms in materials revealed by electron tomography Nat. Mater. 14 1099-1103 (2015) 78

[15] B. Chu and B.S. Hsiao, Small-angle X-ray scattering of polymers, Chem. Rev. 101 1727-1761 (2001) 78

[16] M. Megens, C.M. van Kats, P. Bösecke, and W.L. Vos, In situ characterization of colloidal spheres by synchrotron small-angle $X$-ray scattering, Langmuir 13, 6120-6129 (1997) 78

[17] A.G. Shabalin, J.-M. Meijer, R. Dronyak, O. M. Yefanov, A. Singer, R.P. Kurta, U. Lorenz, O.Y. Gorobtsov, D. Dzhigaev, S. Kalbfleisch, J. Gulden, A. V. Zozulya, M. Sprung, A. V. Petukhov, and I. A. Vartanyants, Revealing three-dimensional strcuture of an individual colloidal crystal grain by coherent X-ray diffrective imaging, Phys. Rev. Lett. 117, 138002: 1-6 (2016) 78

[18] P.C.J. Donoghue, S. Bengtson, X.-p. Dong, N.J. Gostling, T. Huldtgren, J.A. Cunningham, C. Yin, Z. Yue, F. Peng, and M. Stampanoni, Synchrotron $X$-ray tomographic microscopy of fossil embryos, Nature (London) 442, 680683 (2006) 78

[19] A. Sakdinawat and D. Attwood, Nanoscale X-ray imaging, Nature Photon. 4, 840-848 (2010) 78

[20] B. Pollak, Experiences with planography Dis. Chest. 24, 663-669 (1953) 78

[21] P. Cloetens, W. Ludwig, J. Baruchel, D. van Dyck, J. van Landuyt, J.P. Guigay, and M. Schelenker, Holotomography: Quantative phase tomography with micrometer resolution using hard synchrotron radiation $x$ rays, Appl. Phys. Lett. 75, 2912 (1999) 78, 79

[22] C. S. Schroer, J. Meyer, M. Kuhlmann, B. Benner, T. F. Gunler, B. Lengeler, C. Rau, T. Weitkamp, A. Snigirev, and I. Snigireva, Nanotomography based on hard $x$-ray microscopy with refractive lenses Appl. Phys. Lett. 81, 1527-1529 (2002) 78

[23] C. A. Larabell and M. A. L. Gros, X-ray Tomography Generates 3-D Reconstructions of the Yeast, Saccharomyces cerevisiae, at 60-nm Resolution Mol. Biol. Cell 15, 957-962 (2003) 78

[24] R. Mokso, P. Cloetens, E. Maire, W. Ludwig, and J.-Y. Buffire, Nanoscale zoom tomography with hard $x$ rays using Kirkpatrick-Baez optics Appl. Phys. Lett. 90, 144104 (2007) 78, 79

[25] A. Pogany, D. Gao, and S. W. Wilkins, Contrast and resolution in imaging with a microfocus x-ray source Rev. Sci. Instrum. 68, 2774-2782 (1997) 78

[26] P. Kirkpatrick and A.V. Baez, Formation of optical images by X-rays, J. Opt. Soc. Am. 38, 766-774 (1948) 79

[27] S. Zabler, P. Cloetens, J.-P. Guigay, J. Baruchel, and M. Schlenker, Optimization of phase contrast imaging using hard $x$ rays Rev. Sci. Instrum. 76, 073705:1-7 (2005) 80, 82

[28] J.-P. Guigay, Fourier transform analysis of Fresnel diffraction patterns and in-line holograms Optik (Shtuttgart), 49, 121-125 (1977) 80

[29] A. V. Bronnikov Theory of quantitative phase-contrast computed tomography J. Opt. Soc. Am. A., 19, 472-480 (2002) 81

[30] A.N. Tikhonov, On the stability of inverse problem, Dokl. Acad. Nauk SSSR, 39, 195-198 (1943) 82 
[31] D. Paganin, S. C. Mayo, T. E. Gureyev, P. R. Miller, S. W. Wilkins Simultaneous phase and amplitude extraction from a single defocused image of a homogeneous object, J. Microsc., 206, 33-40 (2002) 84

[32] M. R. Teague Deterministic phase retrieval: a Green's function solution J. Opt. Soc. Am. 73, 1434-1441 (1983) 84

[33] K. M. Ho, C. T. Chan, C. M. Soukoulis, R. Biswas, and M. Sigalas Photonic band gaps in three dimensions: New layer-by-layer periodic structures, Solid State Comm. 89 413-416 (1994) 86

[34] M. Maldovan and E. L. Thomas, Diamond-structured photonic crystals Nature Mater. 3, 593-59 (2004).

[35] M.D. Leistikow, A.P. Mosk, E. Yeganegi, S.R. Huisman, A. Lagendijk, and W.L. Vos, Inhibited spontaneous emission of quantum dots observed in a 3D photonic band gap, Phys. Rev. Lett. 107, 193903 (2011). 87

[36] J. M. van den Broek, L. A. Woldering, R. W. Tjerkstra, F. B. Segerink, I. D. Setija, and W. L. Vos, Inverse-woodpile photonic band gap crystals with a cubic diamond-like structure made from single-crystalline silicon, Adv. Func. Mater. 22, 25-31 (2012) 86, 100

[37] http://www.esrf.eu/UsersAndScience/Experiments/XNP/ID16A ） (on 160310) 88

[38] http://henke.lbl.gov (on 170510) 89

[39] M.S. Nixon and A.S. Aguado Feature Extraction and Image Processing (Newnes, Oxford, 2002) 93

[40] L. A. Woldering, R. W. Tjerkstra, H. V. Jansen, I. D. Setija, and W. L. Vos, Periodic arrays of deep nanopores made in silicon with reactive ion etching and deep UV lithography Nanotechnology 19, 145304:1-11 (2008) 100

[41] A.F. Koenderink, A. Alú, and A. Polman Nanophotonics: Shrinking lightbased technology Science 348, 516-521 (2015)

[42] J. E. G. J. Wijnhoven, L. Bechger, and W. L. Vos Fabrication and characterization of large macroporous photonic crystals in titania Chem. Mater. 13 4486-4499 (2001)

[43] W. L. Vos, M. Megens, C. M. van Kats, and P. B'osecke X-ray diffraction of photonic colloidal single crystals Langmuir 13, 6004-6008 (1997) 
X-ray tomography 


\section{CHAPTER 6}

\section{Reflectivity of silicon photonic crystals with and without embedded point defects}

To probe and evaluate optical properties of photonic crystals we discuss broadband microscopic reflectivity measurements on twodimensional (2D) and three-dimensional (3D) photonic crystals. We present the broadband optical microscopy setup that is used. We study reflectivity spectra of $3 \mathrm{D}$ inverse woodpile diamond-like photonic band gap crystals. We identify the spectral features that match with stop gaps obtained from theory. We investigate the effect of embedding controlled defects in a $3 \mathrm{D}$ photonic band gap crystals. We also study in detail the polarization behavior of light in $2 \mathrm{D}$ photonic crystals that are the parent structures of the $3 \mathrm{D}$ crystals. We investigate the angular dependence of both the incident and the reflected polarization while these polarizations are mutually parallel or perpendicular. We find evidence for strong birefringence in the $2 \mathrm{D}$ stop gap.

\subsection{Introduction}

To achieve desired control over light propagation beyond designing and fabricating photonic crystal structures it is necessary to evaluate their optical performance and understand their optical properties. Depending on the functionality of the structure different experiments serve to evaluate its performance [1-3]. Nevertheless it remains a challenging task to experimentally demonstrate the presence of a complete photonic band gap in a sample. One possibility is to place light emitters inside the crystal and study the spontaneous emission spectra $[2,3]$. The great advantage of such approach is that it is free from theory assumptions and directly probes the local density of states (LDOS) inside the structure. The difficulties with spontaneous emission measurements are that it is hard to control the spatial position of the emitters inside the crystal and filter the signal from the emitters located outside the crystal. Another possibility to verify the presence of stop gaps and band gaps is to measure light reflected from or transmitted through the photonic crystal. In the presence of the stop gap, light with a certain incident wave vectors $k$ will be reflected since it can not propagate inside the crystal. When light is tuned to the frequency range of the $3 \mathrm{D}$ photonic band gap, light will be reflected for any incidence $k$ vector. The 
disadvantage of such approach is the difficulty of the interpretation of reflectivity and transmission spectra. One needs a complicated theory to compare and interpret experimental results and differences occur from the fact that calculated geometries inevitably differ from the real structures. Moreover stop bands may occur in the reflectivity or transmission due to so-called silent modes. These modes appear when the incident plane waves can not couple to the modes inside the crystal and thus being reflected.

One of the properties of the stop gaps is that the wave vector $\mathbf{k}$ has a complex value inside the stop gap $\mathbf{k}=\mathbf{k}^{\prime}+j \mathbf{k}^{\prime \prime}$ where the imaginary part is caused by Bragg interference [4]. Thus light in the stop gap is attenuated and there is less intensity in the transmission (trough) and conversely there is more intensity in the reflection (peak). To prove the presence of 3D band gap one needs to study all possible incident $\mathbf{k}_{\mathbf{i n}}$ from 0 to $2 \pi$. This requirement means that one needs to have the $N A$ of the incident objective $N A_{i n}=1.0$. Practically the consideration arises how large $N A$ can be achieved for reflectivity measurements and how large $N A$ is enough to be representative.

In this Chapter we concentrate on 3D inverse woodpile structures due to their large predicted relative band gap $\frac{\Delta \omega}{\omega_{c}}=25 \%$ [5]. Previous reflectivity and transmission studies performed on the similar structures are reported in the references [6] on the $\mathrm{TiO}_{2}$ infiltrated woodpile structures with nearly perfect reflectance, [7] on the direct $\mathrm{Si}$ woodpile structure, [8] on the transmission measurements of 3D photonic crystals based on macroporous silicon and [16] on the reflectivity measurements of $\mathrm{Si}$ inverse woodpile photonic crystals with similar structure to the samples under investigation in this chapter but surrounded by the large array of $2 \mathrm{D}$ photonic crystal.

In the present work we study $3 \mathrm{D}$ inverse woodpile photonic crystal with lateral extend $\Delta X \Delta Y \approx 10 \times 10 \mu \mathrm{m}$ surrounded by quasi-infinite high refractive index medium ( $\mathrm{Si}$ ) from 4 sides and low refractive index medium (air) from 2 other sides. The extend in $\Delta Z$ direction (thickness) is less significant as is reported in the Reference [9] and has negligible affect on the crystal reflectivity. Due to the finite size in $\Delta X \Delta Y$ directions we need to precisely focus laser light on the surface of $3 \mathrm{D}$ photonic crystal and thus employ microscopy setup to perform reflectivity measurements. To study large range of $\mathbf{k}$ vectors we focus light with high $N A$ objective. Due to the broad width of stop gaps under study we need to carefully select optics used in the experiments and perform spectroscopic measurements for frequency resolution.

The parent $2 \mathrm{D}$ structure of $3 \mathrm{D}$ inverse woodpile is centered rectangular $2 \mathrm{D}$ array of deep pores. Since the array of pores is highly anisotropic, it is relevant to wonder about polarization behavior of light reflected from $2 \mathrm{D}$ photonic crystal. Previously a large birefringence of 0.366 below the first photonic band edge at the wavelength of $6.52 \mu \mathrm{m}$ was reported by F. Genereux et al [10] in the triangular lattice crystal of air cylinders in silicon. Birefringence in their study is defined as a difference in the effective refractive indices of the electric fields polarized parallel and perpendicular to the cylinder axis. Here we propose a polarizationresolved study of our $2 \mathrm{D}$ photonic crystals where we rotate polarization and keep analyzer parallel or perpendicular to polarizer. 


\subsection{Optical setup for broadband reflectivity}

For measurements of the reflectivity spectra from our nanophotonic structures an optical reflectivity setup was built based on previous versions[11]. Since we are interested in the optical properties of fabricated structures both inside and outside of the predicted band gaps and stop gaps, the reflectivity needs to be measured in large range of optical frequencies thus a broad band light source is required. For our samples the expected stop gaps are located in the near infrared (NIR) part of the spectrum (4000- $\left.10000 \mathrm{~cm}^{-1}\right)$. This imposes the requirements on the optical components of the setup to be dispersionless throughout the whole range of interest. In order to avoid chromatic aberration the entire optical setup should be built without use of lenses but with reflecting optics. For focusing and expanding of the beam curved mirrors are used.

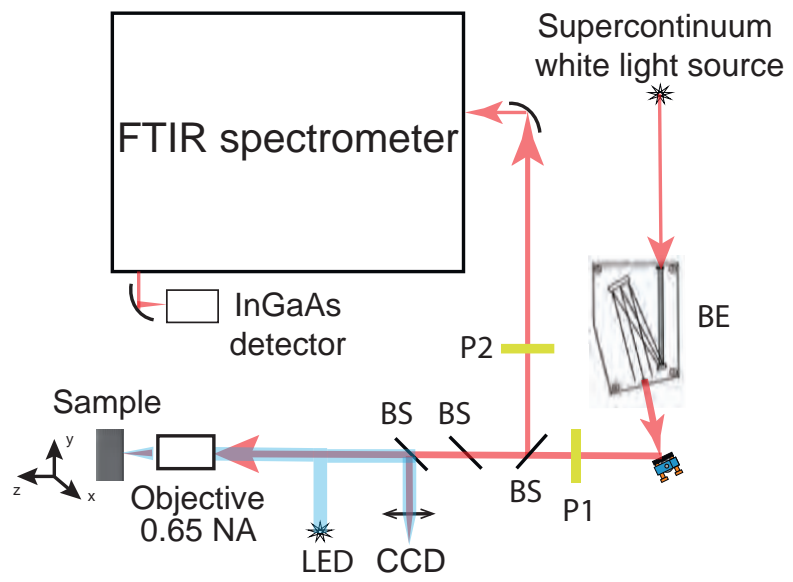

Figure 6.1: Schematic of the microscopic optical reflectivity setup. The beam of the broadband supercontinuum white light source is expanded with reflective beam expander $\mathrm{BE}$ and focused onto the sample using a dispersionless reflective microscope objective with $\mathrm{NA}=0.65$. The signal is reflected from the sample and analyzed using an FTIR spectrometer and InGaAs detector. For imaging of the sample surface the light from the LED illuminates the sample and then focused onto a CCD camera. Incidence and reflected beams are separated with beam splitter BS. P1 polarizes the incident beam and $\mathrm{P} 2$ analyzes the polarization of the reflected beam.

The scheme of the setup is shown in the Figure 6.1. It consists of a supercontinuum source, a beam expander (BE), a high numerical aperture (NA) reflecting objective and a Fourier-Transform infrared (FTIR)spectrometer equipped with an external detector. The supercontinuum source covers the spectral range from $4000 \mathrm{~cm}^{-1}$ to $22000 \mathrm{~cm}^{-1}$. The beam from the supercontinuum white laser source is collimated at the fiber output of the source and spatially filtered with an adjustable iris to select the central part with the most uniform spectral distribution. $96 \%$ of the laser power is dumped and the beam is expanded with the reflective beam expander BE (Thorlabs BE06R) to cover fully the pupil of 
the reflective objective (Ealing $74 \mathrm{x}$ ) with a numerical aperture $\mathrm{NA}=0.65$. The full width at half maximum (FWHM) of the focus was measured by scanning along the sharp edge of wafer and was found to be around $4 \mu \mathrm{m}$. The sample was mounted on an $(x, y, z)$ stage with piezomotors (Auttocube ECC100) for precise positioning. In between the measurements we observed the position of the beam on the sample with the white LED illumination and the CCD camera. A polarizer and analyzer Thorlabs LPNIRA050 are used to investigate the polarization effects. The reflected signal is analyzed using the FTIR spectrometer Biorad FTS6000 with a resolution of $8 \mathrm{~cm}^{-1}$ and detected with an external InGaAs detector Teledyne Judson J23. The electric output of the detector was fed to the spectrometer electronics via a preamplifier and filter Stanford Research Systems SR560. All reflectivity spectra were calibrated by taking the ratio of the measured spectra to a reference spectrum that was measured on a gold mirror before and after the measurements using the same experimental conditions as the photonic structure under investigation. We also took reflectivity spectra of bulk silicon near the photonic crystal structure that were sometimes used as reference spectra in case there was no time to take gold reference spectra.

\subsection{Reflectivity of 2D silicon photonic crystals}

We have studied one 2D photonic crystal sample with internal sample name in Ad3a-14112008. The crystal consists of an array of deep pores etched in silicon using the Bosch reactive ion etching process [12]. The SEM image of the 2D photonic crystal is shown in Figure 6.2. The pores are etched in the $X$ direction. The pore depth is $6 \mu \mathrm{m}$ and the pore radius at half depth is $r=130 \pm 5 \mathrm{~nm}$. Thus the reduced pore radius equals $r / a=0.19$, which determines the photonic band structure[12]. The pores are ordered in a centered rectangular lattice in $Z Y$ plane with lattice parameters $a=690 \mathrm{~nm}$ and $c=480 \mathrm{~nm}$ and ratio $c / a=\sqrt{2}$. The high-symmetry $\Gamma K$ direction of light propagation coincides with the $Z$ axis. In Reference [13] the reflectance of similar 2D photonic crystals is studied for TEand TM-polarized light. 2D photonic crystals exhibit different reflectance for TE- and TM-polarized light but the detailed study of the polarization dependent reflectivity was not yet reported.

Here we address the polarization behavior of $2 \mathrm{D}$ photonic crystals in the following way. Two sets of experiments are performed. In the first set the analyzer is set parallel to the polarizer that sets the incidence polarization. The incident polarization $\theta$ of the $\mathbf{E}$ field is set with respect to the $X$ axis as shown in the Figure 6.2. Both polarizer and analyzer are rotated by the same angle $\theta$ and the reflectivity is measured for each polarizer angle $\theta$. Thus if the incident polarization is set to $\theta_{P}=45^{0}$ the analyzer is also set $\theta_{A}=45^{0}$. We refer to this case as "parallel polarizer" case. In the second set of experiments, called "crossed polarizer", the analyzer is set perpendicularly to the incidence polarization angle $\theta$. Thus if the polarizer is set to $\theta_{P}=45^{0}$, the analyzer is set to $\theta_{A}=125^{0}$. The reflectivity spectra are recorded for incidence polarization angles between 0 and $90^{\circ}$ with 5 degrees step for both cases. 


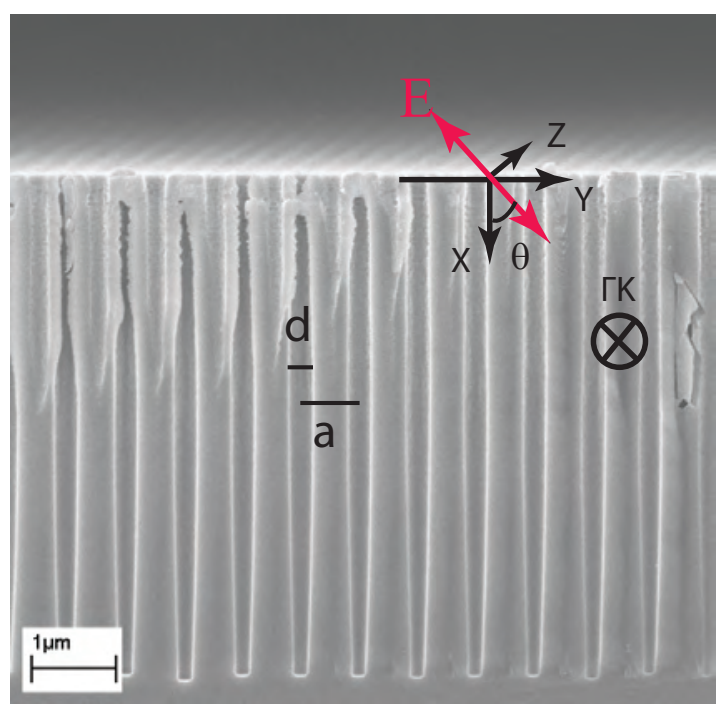

Figure 6.2: SEM image of the $2 \mathrm{D}$ photonic crystal under investigation. Deep pores in silicon are running along the $X$ axis and are arranged in the centered rectangular lattice in the $Z Y$ plane with the lattice parameters $a=690 \mathrm{~nm}, c=480 \mathrm{~nm}$ and pore diameter $d=260 \mathrm{~nm}$. The angle of incident polarization of the electric field $\theta$ is measured starting from the $Y$ axis as shown in the picture. The propagation direction of the axis of the incoming beam is parallel to the $\Gamma \mathrm{K}$ high symmetry direction.

Figure 6.3 shows reflectivity measured in the parallel polarizer case. The $\theta=0$ polarization case (6.3(a)) corresponds to the TM polarization for which stop gap frequencies were calculated earlier in Reference [13]. The first observed reflectivity peak is centered at $5027 \mathrm{~cm}^{-1}$ and agrees well with the calculated stop gap in $\Gamma K$ direction centered at $4997 \mathrm{~cm}^{-1}$ and extending from 4882 to $5112 \mathrm{~cm}^{-1}$. The second observed reflectivity peak centered at $6268 \mathrm{~cm}^{-1}$ may correspond to the stop gap in the $\Gamma B$ direction that extends outside the Brillouin zone and was not a part of the band structure calculation. The third observed reflectivity peak is centered at $9028 \mathrm{~cm}^{-1}$ and agrees well with the calculated stop gap centered at $8771 \mathrm{~cm}^{-1}$ and extending from 7812 to $9731 \mathrm{~cm}^{-1}$. Figure $6.3(\mathrm{c})$ shows the TE polarized reflectivity spectra. The first observed reflectivity peak is centered at $4722 \mathrm{~cm}^{-1}$ and does not correspond to any known stop gap in the band diagram. The second wide reflectivity peak observed with the center at $5899 \mathrm{~cm}^{-1}$ agrees reasonably well with the calculated stop gap centered at $6187 \mathrm{~cm}^{-1}$ and extending from 5110 to $7265 \mathrm{~cm}^{-1}$. This stop gap is the sub-Bragg gap that was first identified in Reference [13]. The third and the fourth reflectivity peaks centered at 8289 and $9828 \mathrm{~cm}^{-1}$ respectively do not match known stop gaps in the band diagram for $\Gamma \mathrm{K}$ direction, although they might be explained with calculations of the band structure beyond $\Gamma \mathrm{K}$ direction. The fifth reflectivity peak centered at $10610 \mathrm{~cm}^{-1}$ matches well with the calculated stop gap centered at $10755 \mathrm{~cm}^{-1}$ and extending from $10010 \mathrm{~cm}^{-1}$ to $11500 \mathrm{~cm}^{-1}$. Figure 6.3(b) shows the reflec- 


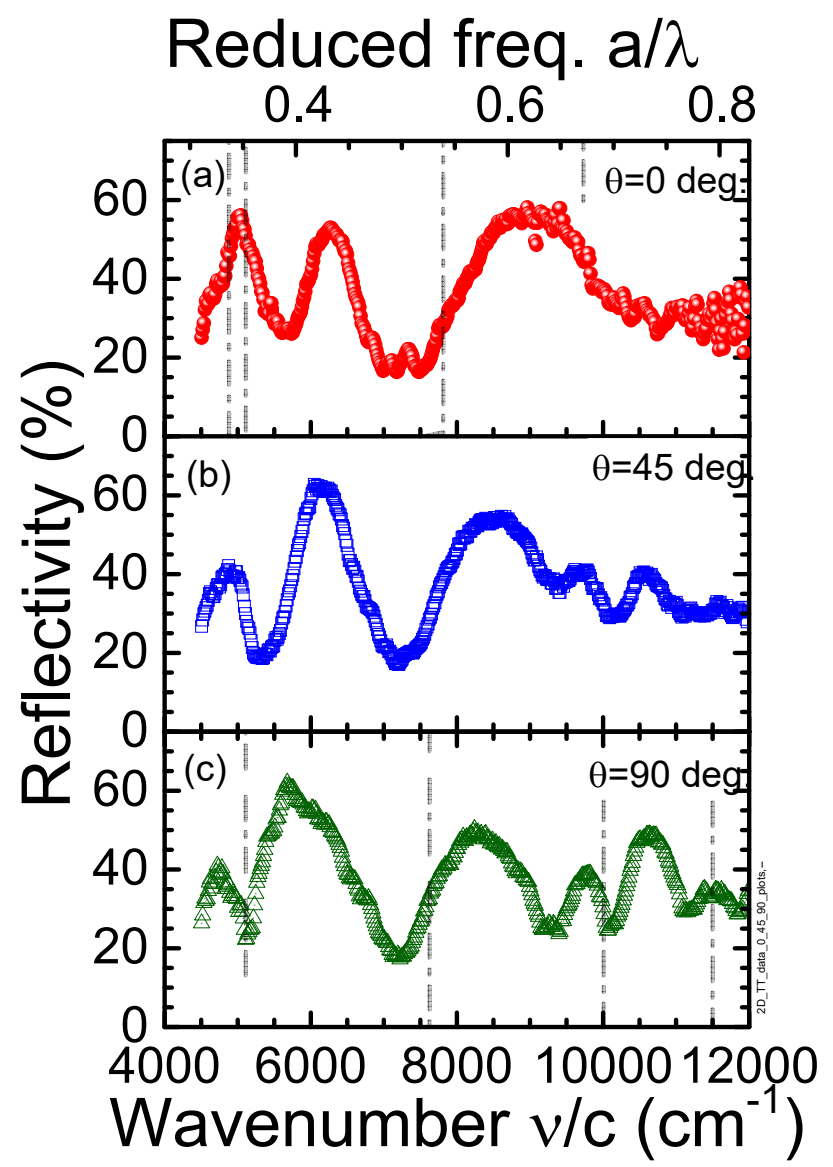

Figure 6.3: Reflectivity measured from a 2D photonic crystal Ad3a-14112008 in case of parallel polarizer for incidence polarization angles $\theta=0^{\circ}$ (TM mode) (a), $\theta=45^{\circ}$ (b) and $\theta=90^{\circ}$ (TE mode) (c). Gray bars indicate the extent of the calculated stop gaps.

tivity spectrum collected with polarizer set to $\theta=45^{\circ}$, intermediate between the TE and the TM incident polarizations. The spectrum exhibits features common for both TE and TM reflectivity spectra such as peaks centered at 4910, 6185, 8458,9786 and $10583 \mathrm{~cm}^{-1}$, yet shifted in frequency.

Figure 6.4 shows a reflectivity map in parallel polarizer case measured for incident angles between 0 and $90^{\circ}$. It is clear from the plot that high reflectivity peaks decrease in frequency when the incidence polarization angle $\theta$ increases. Two most pronounces reflectivity peaks centered around 6000 and $7000 \mathrm{~cm}^{-1}$ are present for every measured incident polarization angle and are changing in the bandwidth. Narrower reflectivity peaks centered around 5000 and $11000 \mathrm{~cm}^{-1}$ are only present for a range of incident polarization angles from 0 to 45 degrees 


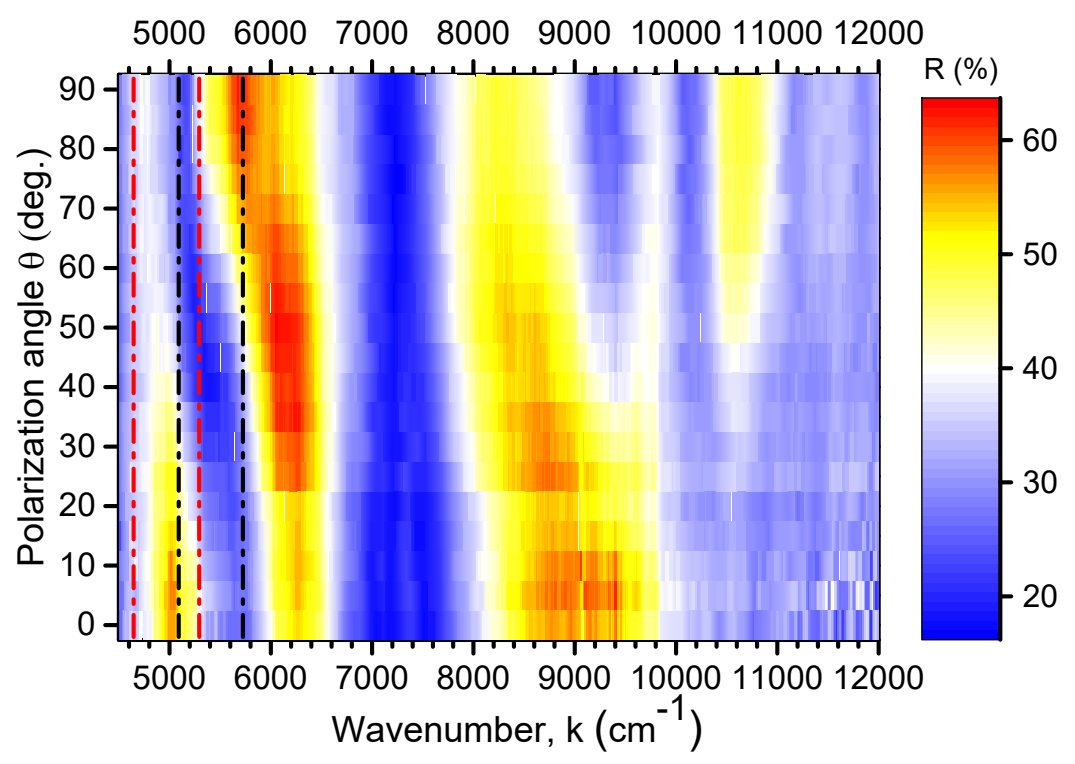

Figure 6.4: Reflectivity of $2 \mathrm{D}$ photonic crystal at the different angles of polarizer $\theta$ in the parallel polarizer case. The color scale represents the reflectivity value. On the horizontal axis is the wavenumber in $\mathrm{cm}^{-1}$ and on the vertical axis is the incidence polarization angle. The incident direction of the beam propagation is $\Gamma K$. Dashed lines indicate frequencies where reflectivity cross sections were taken for the plots in Figures 6.8 and 6.7 .

and from 45 to 90 degrees respectively.

The next set of reflectivity measurements is done for the crossed polarizer case. Figure 6.5 shows reflectivity spectra collected in the crossed polarizer case. It is clear from the plot that for purely TE and TM incident polarizations ( 0 and 90 degrees polarizer angles) the reflectivity detected after the perpendicular analyzer is zero, which is expected for $2 \mathrm{D}$ photonic crystals since TE and T M are strictly separated [4]. However with polarizer set to $\theta=45$ degree a reasonable reflectivity peak of $34 \%$ is observed at $5289 \mathrm{~cm}^{-1}$. This frequency nearly corresponds to the center of the stop gap observed with parallel polarizer described above and confirmed with the band structure calculations. With crossed polarizers it is expected from the Fresnel laws of reflection and refraction [17] that the reflectivity vanishes for isotropic media at normal incidence. Non-zero reflectivity is observed only when the medium is anisotropic and the refractive index tensor has at least two different components $n_{1} \neq n_{2}$. From observed in our experiment reflectivity peak of more than $35 \%$ in crossed polarizers case we conclude that our 2D photonic crystal exhibits large birefringent behavior.

Figure 6.6(a) shows the reflectivity map measured versus incidence polarization angle for the case of crossed polarizer. It is seen that a reflectivity peak of up to $35 \%$ appears in the range between 5125 and $5746 \mathrm{~cm}^{-1}$ at $\theta$ between 20 and $70^{\circ}$. 


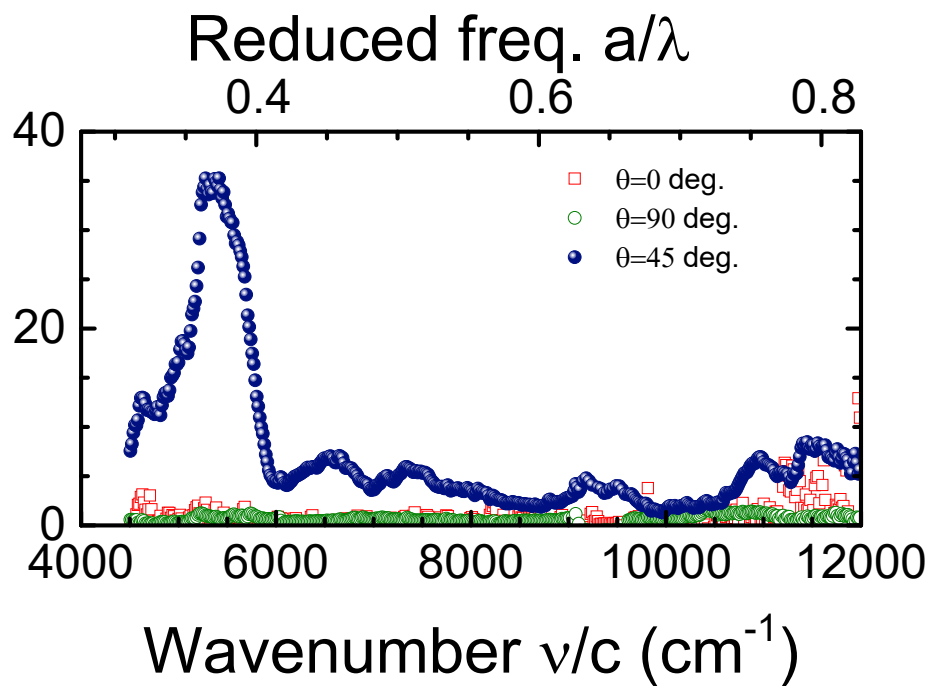

Figure 6.5: Reflectivity measured from $2 \mathrm{D}$ photonic crystal in case of crossed polarizer for the $\theta=0^{\circ}$ (TM mode) (red), $\theta=45^{\circ}$ (blue) and $\theta=90^{\circ}$ (TE mode) (green) incidence polarization angle.

Less intense reflectivity peaks are also observed at higher frequencies 6584,7338 , 9155, 10938 and $11417 \mathrm{~cm}^{-1}$ in the same range of incidence polarization angles. The presence of a strong reflectivity peak in the frequency range of the stop gap in crossed polarizer configuration reveals that $2 \mathrm{D}$ photonic crystal behaves as a birefringent media inside the stop gaps.

To interpret the results that $2 \mathrm{D}$ photonic crystal exhibits birefringent behavior a model was designed. In our model we describe the crystal as a semi infinite slab of effective medium with the interface perpendicular to the $Z$ axis with a refractive index tensor equal to $n=\left[\begin{array}{ccc}n_{1} & 0 & 0 \\ 0 & n_{2} & 0 \\ 0 & 0 & n_{3}\end{array}\right]$. Thus there are two independent components of the refractive index tensor $n_{1}$ and $n_{2}$. We calculate the reflectance from the effective medium using the Fresnel coefficients derived in the Appendix C. We distinguish the two cases of parallel and crossed polarizers by considering separately TE- and TM-polarized components of the incidence wave and projecting the reflected wave on the axis of the analyzer. Thus we obtain the reflectivity of the semi infinite birefringent medium depending on the incident polarization angle for parallel and crossed polarizer cases. Then we apply a least square minimization of our model to the experimental data at each frequency and each incidence polarization angle. The resulting parameters are the effective refractive indices, whose dispersion is obtained $n_{1}$ and $n_{2}$. In this first attempt to interpret the data we prefer to keep the model analytical and to minimize 


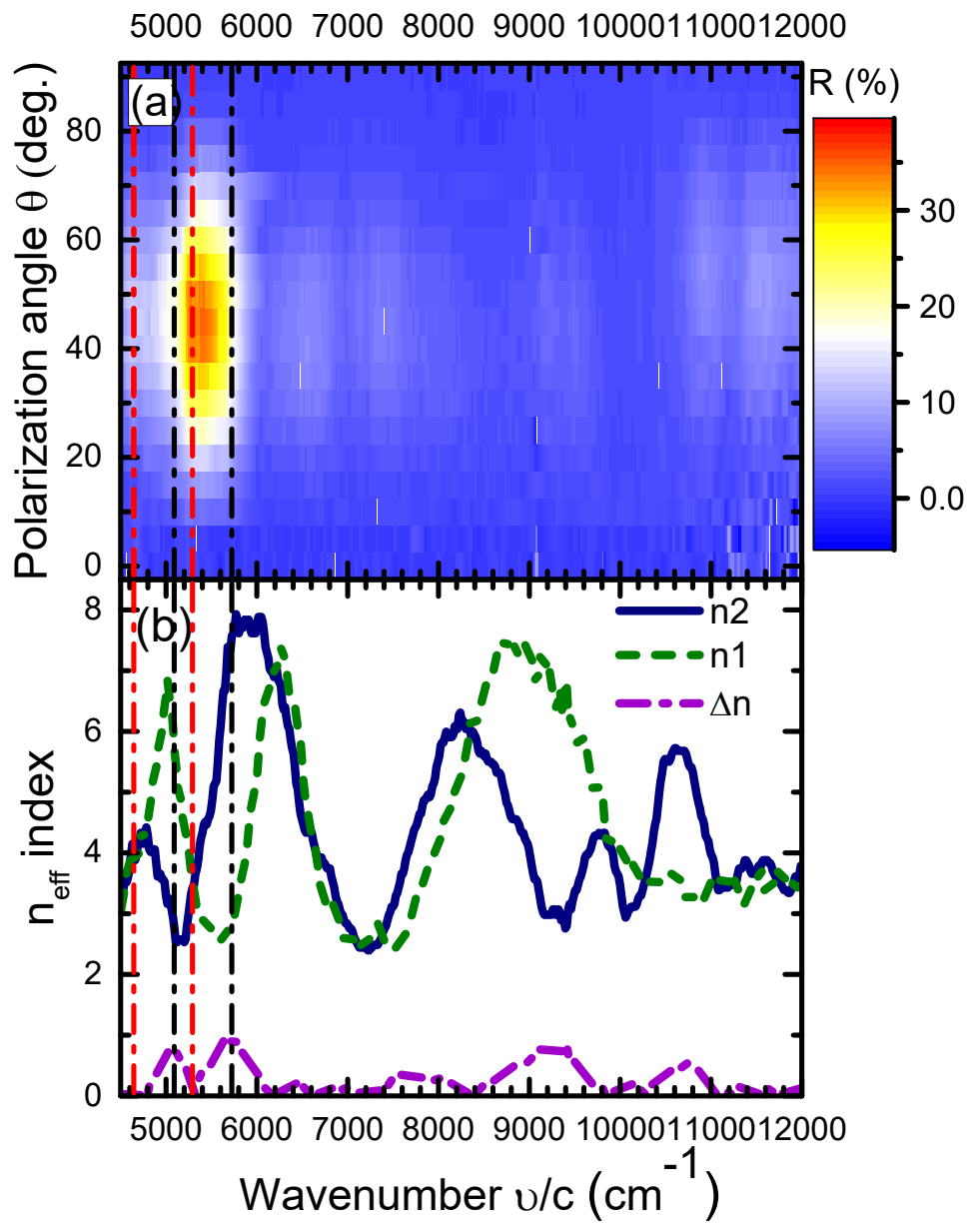

Figure 6.6: (a) Reflectivity map measured fan a $2 \mathrm{D}$ photonic crystal versus angle of incident polarization of the $E$ field and frequency. Incident and detection polarizers are crossed. High reflectivity is observed for a range of frequencies indicating the birefringent nature of the media. (b) Components $\left(n_{1}, n_{2}\right)$ of the effective refractive index tensor extracted from the model. Green dashed line shows $n_{1}$ and blue solid line shows $n_{2}$. Purple dotted line is the absolute normalized difference between $n_{1}$ and $n_{2}$. Dashed lines indicate frequencies at which reflectivity dependence on the angle of incidence polarization is plotted in Figures 6.8 and 6.7.

the number of adjustable parameters, therefore we restrict the refractive index components to be real.

Effective refractive index components $n_{1}$ and $n_{2}$ are plotted in Figure 6.6(b). 
In addition we show the absolute normalized difference between $n_{1}$ and $n_{2}$ defined as $\Delta n \equiv 2 \times\left|\frac{n_{1}-n_{2}}{n_{1}+n_{2}}\right|$ to characterize the degree of anisotropy. At low frequencies below the calculated stop gap $\left(\nu / c<4645 \mathrm{~cm}^{-1}\right)$ both components of refractive index $n_{1}$ and $n_{2}$ are equal which means that photonic crystal behaves as an isotropic media. The reflectivity at $4645 \mathrm{~cm}^{-1}$ and the corresponding model curve are shown in Figure 6.7. It is clearly seen from the plot that at this frequency reflectivity does not depend on the incidence polarization angle $\theta$ which agrees with obtained equality of refractive index components.

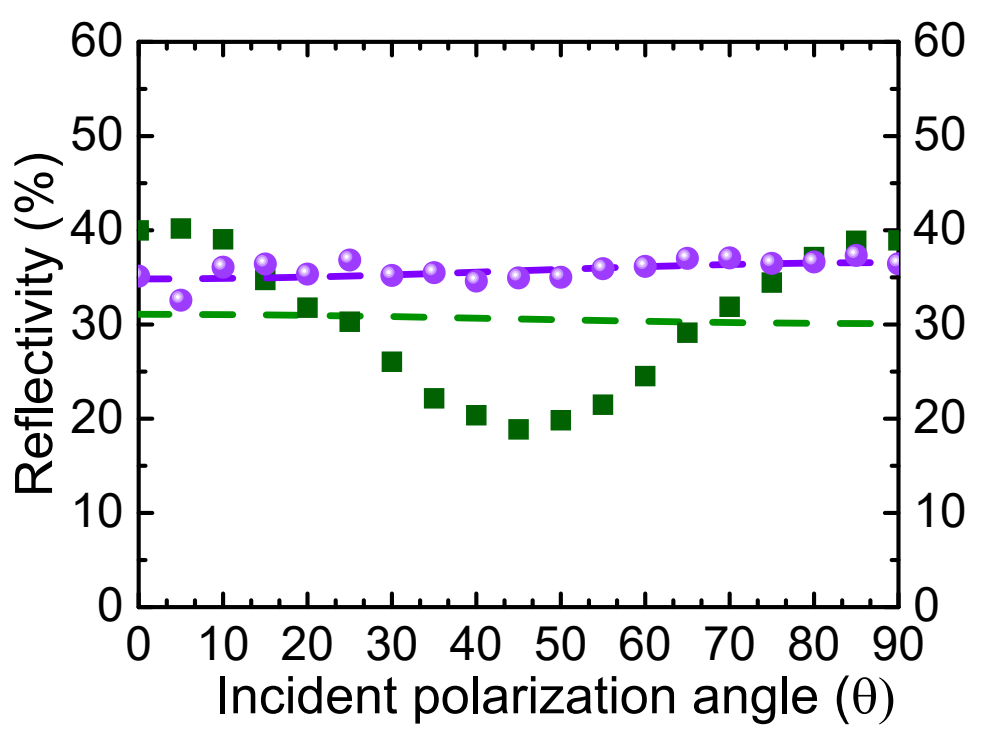

Figure 6.7: Reflectivity of $2 \mathrm{D}$ photonic crystal measured in the parallel polarizer case at different incidence polarization angles $\theta$. Purple symbols shows reflectivity at 4645 $\mathrm{cm}^{-1}$ and green symbols shows reflectivity at $5293 \mathrm{~cm}^{-1}$ as indicated with red dashed lines in Figures 6.4 and 6.6. These frequencies correspond to the smallest anisotropy extracted from the effective media model. Dashed lines show corresponding fit curves from the proposed effective media model.

Above $4800 \mathrm{~cm}^{-1}$ in Figure 6.6(b) a large anisotropy of the refractive index is obtained from the model. Between 4800 and $5293 \mathrm{~cm}^{-1}$ the $n_{x}$ element $\left(n_{1}\right)$ of the refractive index tensor is higher than the $n_{y}$ element $\left(n_{2}\right)$. This can be rationalized with the hypothesis from Bragg stack [4] that waves concentrate their energy mostly in the high refractive index media on the low frequency side of the stop gap, which is in our case Si around the pores. The intuitive picture for the wave energy concentrated in the Si around the pore suggests that in $X$ direction the vector of the electric fiend will oscillate along the long side of the $\mathrm{Si}$ wall and in $Y$ direction the electric field vector will oscillate along the short side of the Si wall between two pores, thus the wave will "feel" the presence of air in the pore with lower refractive index. The reflectivity dependence on the incident 
polarization angle at $5092 \mathrm{~cm}^{-1}$ is shown in Figure 6.8. The high reflectivity when incident polarization is along the $X$ axis $\left(0^{\circ}\right.$ angle of incidence polarization) agrees with the hypothesis above that the wave energy is concentrated in the high refractive index media and thus $x$ component of the refractive index is higher than $y$ component.

At $5293 \mathrm{~cm}^{-1}$ the two components of the refractive index in Figure 6.6(b) are becoming equal $n_{1}=n_{2}$. The reflectivity at $5293 \mathrm{~cm}^{-1}$ and the corresponding model curve are shown in Figure 6.7. While the modeled refractive index components are equal as in the case of isotropic media, the experimental reflectivity as a function of incident polarization differs from the isotropic case. Reflectivity has maximum of $40 \%$ at 0 and $90^{\circ}$ incidence polarization and a minimum of $19 \%$ at $45^{\circ}$. The unusual reflectivity dependence on the incidence polarization indicates that at this frequency an effective medium with two different components of refractive index $\left[n_{1}, n_{2}, n_{2}\right]$ cannot explain the behavior of the photonic crystal.

At frequencies larger than $5293 \mathrm{~cm}^{-1}$ the first component $n_{1}=n_{x}$ of the refractive index has become larger than $n_{2}=n_{y}=n_{z}$ component as shown in Figure 6.6(b). This can be explained with the change of the wave energy distribution to a low refractive index media [4]. If in the high frequency side of the stop gap the wave energy is concentrated more in the low refractive index material (pores), then the $x$ component of the refractive index aligned along the pore depth is lower than $y$ component of the refractive index which is aligned along the short side of the pore and thus "feels" the presence of surrounding Si. This hypothesis is supported with the experimentally observed reflectivity dependence on the incidence polarization angle shown in Figure 6.8. The high reflectivity of $60 \%$ is observed for $90^{\circ}$ incidence polarization angle which means the polarization along the $Y$ direction.

At frequencies larger than $6125 \mathrm{~cm}^{-1}$ Figure $6.6(\mathrm{~b})$ shows that the difference between the components of the effective refractive index becomes close to zero meaning that the medium behaves like in isotropic medium. Above $7329 \mathrm{~cm}^{-1}$ anisotropy of refractive index appears and notably refractive index components alternate which one is larger $n_{1}$ or $n_{2}$ at frequencies 8363,9860 and $10276 \mathrm{~cm}^{-1}$. The variations of the effective refractive index components is most likely associated with the first order Bragg condition and the second order stop gap in 2D photonic crystal.

Thus we observed for the first time large birefringence of $2 \mathrm{D}$ photonic crystals inside the stop gaps. The value of anisotropy $\Delta n$ reaches up to $\Delta n=0.9$ and is up to our knowledge the largest birefringence reported on 2D photonic crystals exceeding more than twice the birefringence below the stop gap reported by $\mathrm{F}$. Genereux et al [10]. The effective refractive indices within the stop gap reaches values up to 8 due to the high reflectivity of $2 \mathrm{D}$ photonic crystal within the stop gap due to the Bragg diffraction. In order to void such unphysical values of the effective refractive indices the model needs to be extended to the complex values of the effective refractive indices to describe the behavior of the photonic crystal withing the stop gap more physically. Moreover, in order to describe the situation when the components $n_{1}$ and $n_{2}$ of the effective refractive index are 


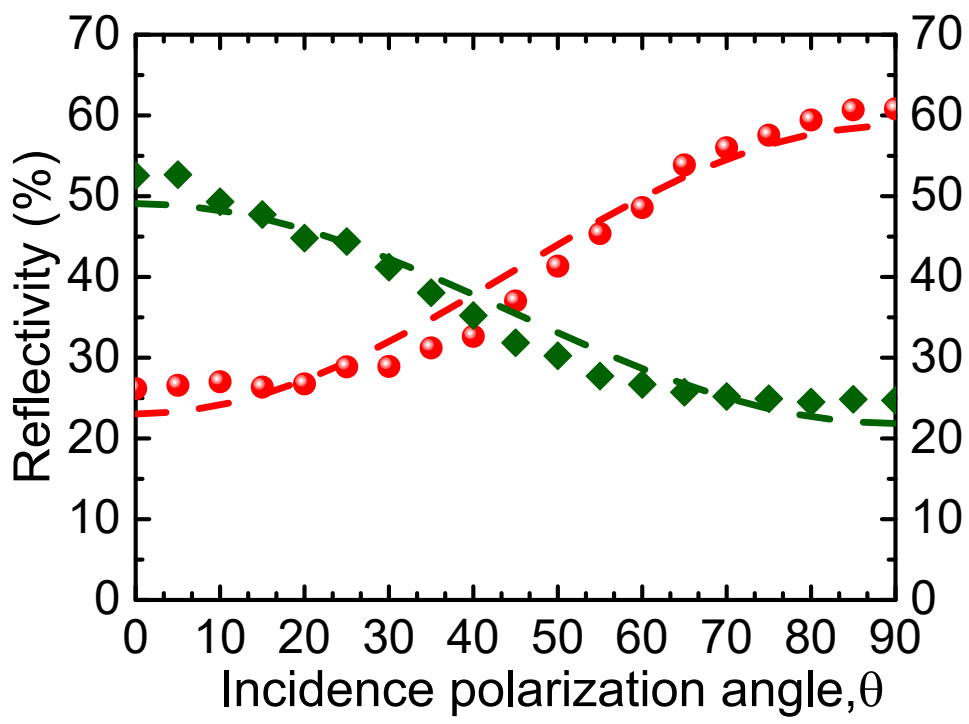

Figure 6.8: Reflectivity of $2 \mathrm{D}$ photonic crystal measured in the crossed polarizer case versus incident polarization angle $\theta$. Green symbols shows reflectivity at $5092 \mathrm{~cm}^{-1}$ and red symbols shows reflectivity at $5725 \mathrm{~cm}^{-1}$ as indicated with black dashed lines in Figures 6.4 and 6.6(b). These frequencies correspond to the largest anisotropy extracted from the effective medium model. Dashed lines show corresponding fit curves from the proposed effective medium model.

found from the model to be the same but the reflectivity nevertheless has strong angular dependence we suggest that effective refractive index tensor might not be diagonal.

\subsection{Reflectivity study of 3D silicon photonic band gap crystals}

\subsubsection{Inverse woodpile photonic crystal}

In this section we consider reflectivity measured on four different $3 \mathrm{D}$ inverse woodpile photonic crystal. The geometry of the inverse woodpile consists of two arrays of cylindrical pores where each arry is arranged in a centered rectangular lattice as shown in Figure 1.2 in Chapter 1. The pore radius $R$ and the lattice parameters $a$ and $c$ are chosen to be $a / c=\sqrt{2}$ such that the 3D crystal structure is face centered cubic with a diamond-like basis [14]. The ratio $R / a$ determines the position and width of the expected band gap according to plane-wave band structure calculations [13] and was varied from sample to sample. We study here reflectivity from two different samples. All samples are listed in Appendix B with 
their lab names, fabrication and measurements history.

First sample under investigation is listed in the Appendix B as Sample D. On the Sample $\mathbf{D}$ we study three structures: $\mathbf{D}_{\mathbf{1}}, \mathbf{D}_{\mathbf{2}}$ and $\mathbf{D}_{\mathbf{3}}$. Structure $\mathbf{D}_{\mathbf{1}}$ is a $3 \mathrm{D}$ photonic inverse woodpile crystal without intended defects thus we refer to such structure design as a quasi-perfect photonic crystal accounting for inevitable fabrication imperfections and finite size. The SEM image of the Structure $\mathbf{D}_{\mathbf{1}}$ as an example of a typical quasi-perfect photonic crystal is shown in Figure 6.9 (a). The design $R / a_{\text {design }}$ ratio for the structure $\mathbf{D}_{\mathbf{1}}$ is 0.23 that is close to the optimum to form the widest band gap according to calculations in reference [12]. Structure $\mathbf{D}_{2}$ by design is equivalent to the Structure $\mathbf{D}_{\mathbf{1}}$ thus we choose this two structures for comparison. Structure $\mathbf{D}_{\mathbf{3}}$ contains the array of the cavities as shown in the Figure $6.9(\mathrm{~b})$. For the regular pores design parameter $R / a_{\text {design }}=0.23$. Each cavity is formed by the intersection of two pores with twice smaller radius $R^{\prime}=1 / 2 R$. Every pore with smaller radius is indicated with the red circle on the picture. Thus there are 80 cavities inside the crystal arranged in a cubic lattice. The third sample studied in this chapter is sample $\mathbf{E}$ (see B for the name in the lab) where we consider on this sample a single 3D photonic crystal Structure $\mathbf{E}_{\mathbf{1}}$ with 4 embedded cavities arranged diagonal along the crystal as is shown in figure $6.9(\mathrm{c})$. Each cavity is formed at the intersection of the smaller pore with radius $R^{\prime}=1 / 2 R$.
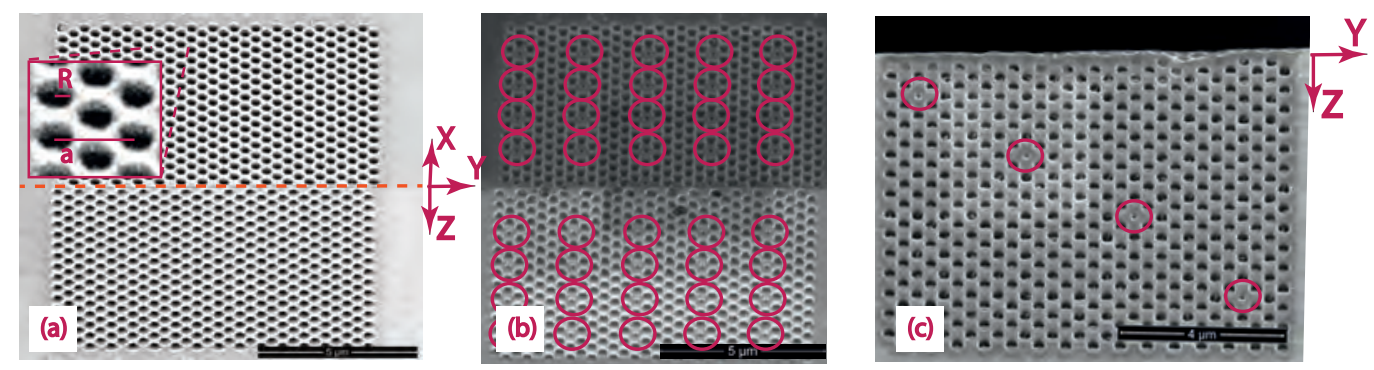

Figure 6.9: SEM image of (a) Structure $\mathbf{D}_{\mathbf{1}}$ as an example of a typical quasi-perfect $3 \mathrm{D}$ photonic crystal without embedded defects, (b) Structure $\mathbf{D}_{\mathbf{3}}$ with $3 \mathrm{D}$ array of cavities based on smaller pores that are indicated with red circles and (c) Structure $\mathbf{E}_{1}$ consisting of 4 cavities diagonally deeper inside the crystal. The dashed red line indicates the 90 degree edge of silicon wafer. Scale bars are shown on the picture.

\subsubsection{Reflectivity of 3D inverse woodpile photonic crystal without embedded defects}

In this section we study reflectivity from Structures $\mathbf{D}_{\mathbf{1}}$ and $\mathbf{D}_{\mathbf{2}}$ that are by design equivalent quasi-perfect $3 \mathrm{D}$ photonic crystals without intended defects with $R / a_{\text {design }}=0.23$. For the broadband reflectivity measurements we use the setup described in a section 6.2 above. Reflectivity of Structure $\mathbf{D}_{\mathbf{1}}$ is shown 
in the Figure 6.10. There are three distinguishable peaks of reflectivity seen in the spectrum. First observed stop band is centered at $5463 \mathrm{~cm}^{-1}$ and extends from $5310 \mathrm{~cm}^{-1}$ to $5617 \mathrm{~cm}^{-1}$. Second stop band is a wide peak from 5876 $\mathrm{cm}^{-1}$ to $7414 \mathrm{~cm}^{-1}$ with the center at $6645 \mathrm{~cm}^{-1}$. Third observed stop band is centered at $8556 \mathrm{~cm}^{-1}$ and extends from $8080 \mathrm{~cm}^{-1}$ to $9032 \mathrm{~cm}^{-1}$. The gap in the spectrum around $9348 \mathrm{~cm}^{-1}$ is a result of filtering out the master source of the supercontinuum white light laser. The maximum reflectivity normalized to the

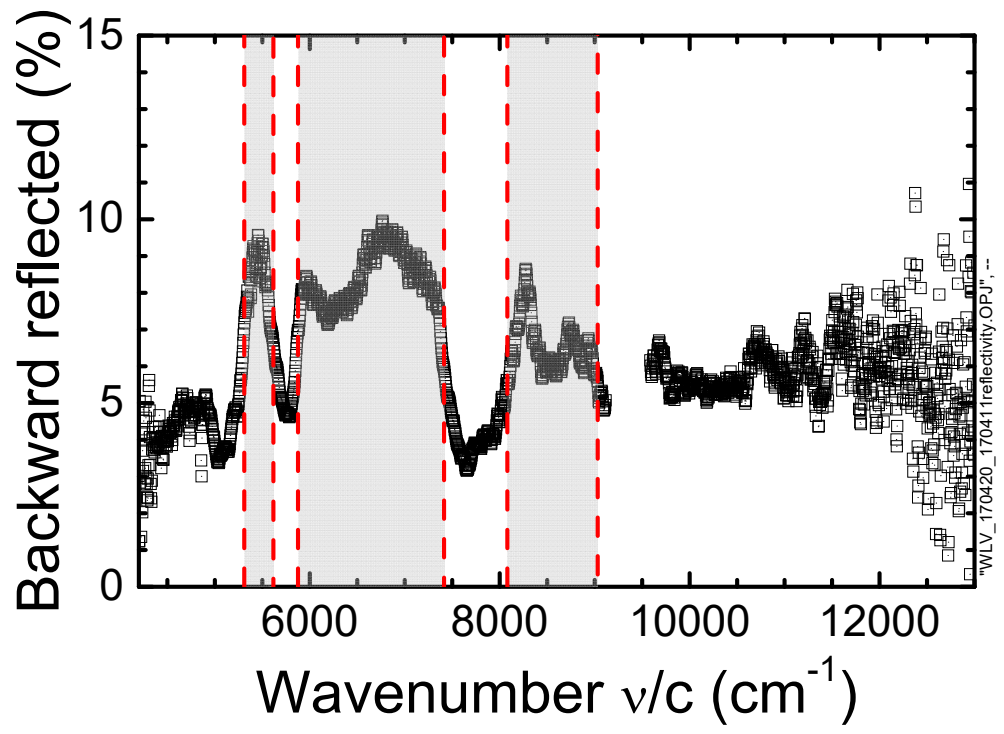

Figure 6.10: Reflectivity of the quasi-perfect photonic crystal Structure $\mathbf{D}_{\mathbf{1}}$ with design $R / a=0.23$ and without embedded defects. The observed stop bands are indicated with the gray bars. The gap in the spectrum around $9339 \mathrm{~cm}^{-1}$ is filtered out to remove artifacts from the pump source of the supercontinuum source.

gold mirror reaches up to $10 \%$. We associate such low reflectivity from the crystal with few aspects. Firstly, the $4 \mu \mathrm{m}$ focus size of a laser beam is comparable to the extent of the 3D photonic crystal determined from tomography measurement $\Delta X=4.6 \mu \mathrm{m}$ and $\Delta Y=3.6 \mu \mathrm{m}$ and since the crystal is located at the edge of Si bar some of the beam intensity leaks above the edge into the air during measurements close to the edge. Secondly, the crystal under investigation is a finite size structure in $\Delta X \Delta Y$ directions surrounded by the quasi-infinite high refractive index medium ( $\mathrm{Si}$ ) from 4 sides and low refractive index medium (air) from 2 sides. Previously reported high reflectivity such as $67 \%$ in the Reference [16] were measured for the 3D photonic crystal surrounded from 3 sides with a quasi-infinite array of $2 \mathrm{D}$ photonic crystal. Thirdly, we can expect a surface roughness to scatter light out of the collecting objective thus decreasing the reflectivity. The band diagram of inverse woodpile photonic crystal depends crucially on the $(R / a)$ ratio of the structure (see Chapter 1 and Reference [16]). 
Figure 6.11 shows the lower and higher band edges calculated in reference [16] and the central frequency $\omega_{c}$. It is clear that the width of the band gap $\Delta \omega$ strongly depends on the $R / a$ ratio. We overlap the observed stop bands from the reflectivity spectrum of the Structure $\mathbf{D}_{\mathbf{1}}$ with the calculated positions of the band edges. From the overlap we can see that observed wide stop band between $5876 \mathrm{~cm}^{-1}$ and $7414 \mathrm{~cm}^{-1}$ is in perfect agreement with calculated band gap width $\Delta \omega$ and central frequency $\omega_{c}$ for the inverse woodpile structure with $R / a_{\text {refl }}=$ $0.196 \pm 0.001$. The $R / a$ ratio extracted from tomography data discussed in the Chapter $5 R / a_{\text {tomo }}=0.197 \pm 0.01$ is in an excellent agreement with the $R / a$ ratio obtained from reflectivity measurements $R / a_{\text {refl }}=0.196 \pm 0.001$.

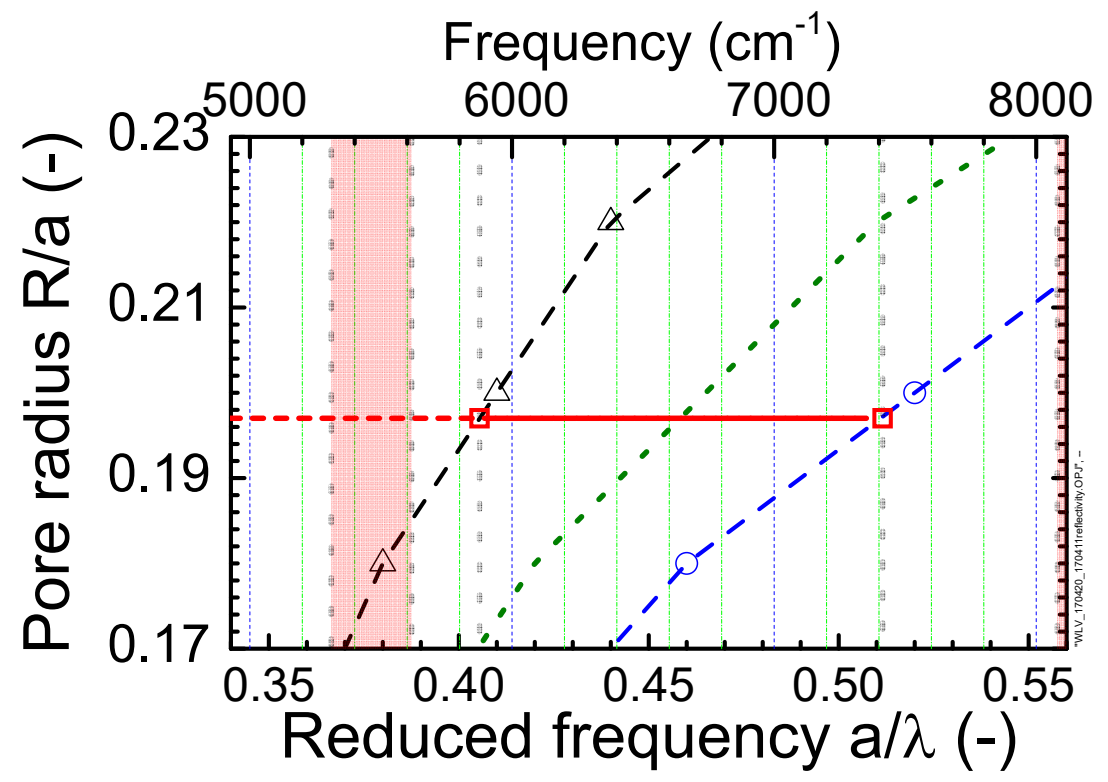

Figure 6.11: Observed stop bands (pink bars) of the Structure $\mathbf{D}_{\mathbf{1}}$ overlapped with the calculated from the band structure positions of the lower (blue) and higher (black) band edges and central frequency $\omega_{c}$ of the inverse woodpile photonic crystal. The band edges are plotted for different $R / a$ ratios.

Figure 6.12 shows the reflectivity measured from the Structure $\mathbf{D}_{\mathbf{2}}$. The structure is an equivalent to Structure $\mathbf{D}_{\mathbf{1}}$ of a quasi-perfect $3 \mathrm{D}$ photonic crystal with the design $R / a_{\text {design }}=0.23$. The reflectivity spectrum exhibits three distinguishable stop bands. The first one is a broad peak centered at $5557 \mathrm{~cm}^{-1}$ and extends from $4848 \mathrm{~cm}^{-1}$ to $6266 \mathrm{~cm}^{-1}$. This stop band appears as a triplet of peaks and is located below the band gap. The second stop band extends from $6678 \mathrm{~cm}^{-1}$ to $8084 \mathrm{~cm}^{-1}$ with the center at $7381 \mathrm{~cm}^{-1}$. The third stop band is centered at 8773 $\mathrm{cm}^{-1}$ and is located in frequency range between 8578 and $8969 \mathrm{~cm}^{-1}$. In Figure 6.13 we overlap the observed stop bands with the band gap edges and central frequency calculated for different $R / a$. It is seen from the Figure 6.13 that the central frequency of the second stop band $\left(6678\right.$ to $\left.8084 \mathrm{~cm}^{-1}\right)$ is matching the 


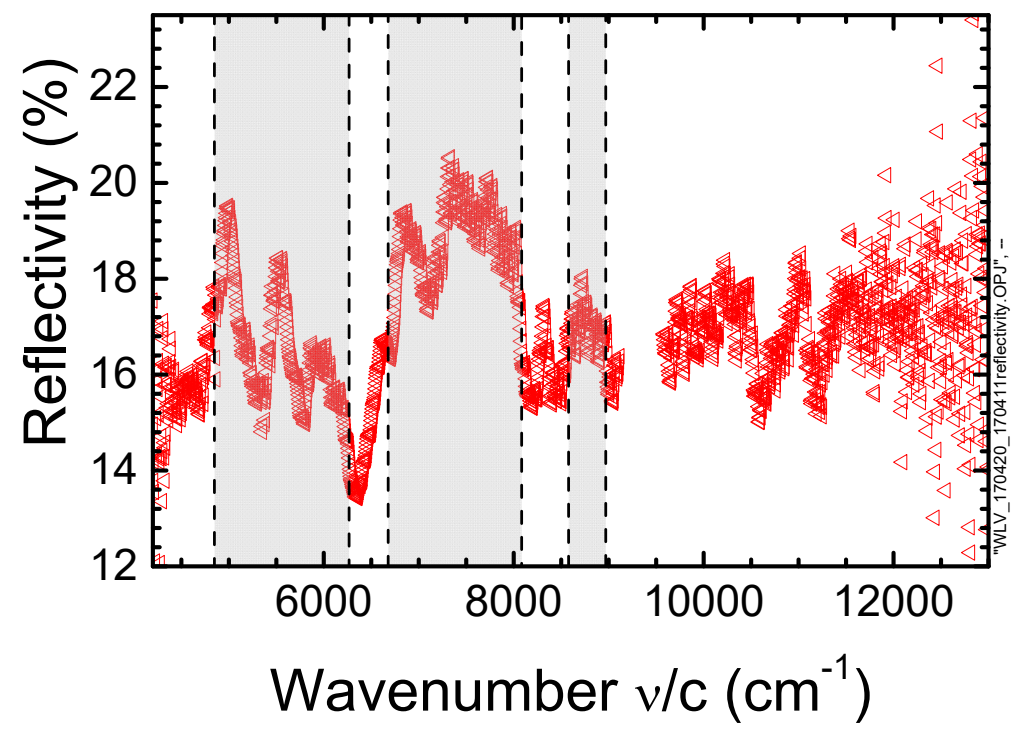

Figure 6.12: Reflectivity of the quasi-perfect photonic crystal Structure $\mathbf{D}_{\mathbf{2}}$ with design design $R / a=0.23$ and without embedded defects on the sample $\mathrm{D}$. The observed stop bands are indicated with the gray bars. The gap in the spectrum around 9339 $\mathrm{cm}^{-1}$ is filtered out to remove artifacts from the pump source of the supercontinuum source.

central frequency $\omega_{c}=7381 \mathrm{~cm}^{-1}$ of the band gap for $R / a_{\text {refl }}=0.22 \pm 0.007$. From X-ray tomography data we obtained $R / a_{\text {tomo }}=0.21 \pm 0.015$ which is in very good agreement with $R / a$ estimated from stop band position. Thus we found two independent ways to determine the $R / a$ ratio of $3 \mathrm{D}$ photonic crystal that are in excellent agreement with each other and therefore strongly supports the interpretation of optical reflectivity spectra. As was discussed in the Chapter 1 one of the characteristics of photonic crystal is photonic strength $S=\frac{\Delta \omega}{\omega_{c}}$. Photonic strength $S$ calculated from the band diagram of inverse woodpile photonic crystal for different $R / a$ is shown with the dashed line in Figure 6.14. From our reflectivity measurements we obtained experimentally the width of the stop band and the central frequency similarly as described above for the range of structures with different $R / a$. Thus we can plot experimental results of the photonic strength $S=\frac{\Delta \omega}{\omega}$ versus $R / a$ (Figure 6.14). It is seen from the Figure 6.14 that one one data point is matching excellently within the error bar the predicted photonic strength. This data point is obtained for the Structure $\mathbf{D}_{\mathbf{1}}$. The remaining data points are lying below the predicted photonic strength curve which can be associated with fabrication imperfections such as tapering of the pores resulting in the stop band narrower than the calculated one. 


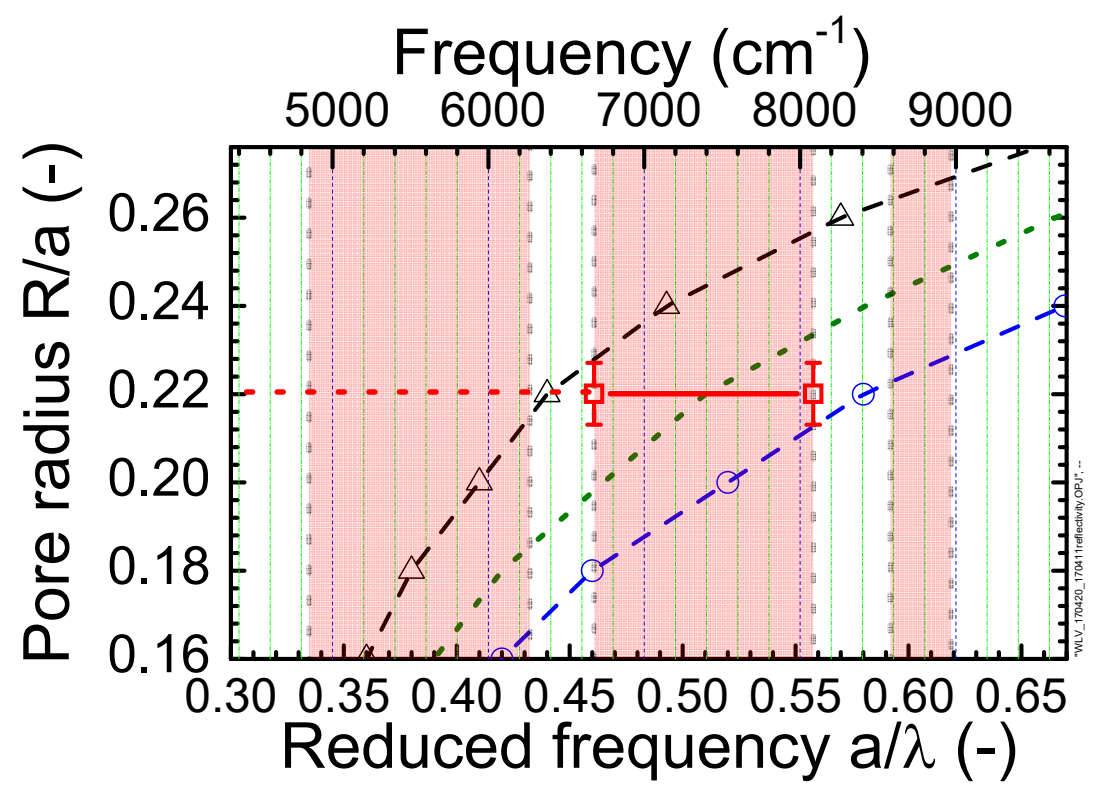

Figure 6.13: Observed stop bands (pink bars) of the Structure $\mathbf{D}_{\mathbf{2}}$ overlapped with the calculated from the band structure positions of the lower (blue) and higher (black) band edges of the inverse woodpile photonic crystal. The band edges are plotted for different $R / a$ ratios.

\subsubsection{Signature of the cavity resonance in the 3D band gap}

In this section we consider reflectivity from photonic crystal structures with embedded defects. The defects are designed as an intersection of two defect pores with radii twice smaller than the regular pore radius $R^{\prime}=R / 2$. According to the supercell band structure calculations in the Reference [15] for the infinitely repeated $5 \times 5 \times 5$ unit cells crystal with two crossing defect pores there are 5 isolated resonances appearing in the band gap the crystal. Considering the finite size of our structures under investigation we may expect a different optical behaviour than an infinite structure. We consider the presence of a trough within the stop band as a signature of a cavity resonance meaning that light propagation occurs inside the stop band. Figure 6.15 shows reflectivity spectra taken at different $X$ positions on the Structure $\mathbf{E}_{\mathbf{1}}$ centered arounf the position of the cavity defect. Every spectrum in Figure 6.15 is plotted with the bias of $10 \%$ from the spectrum taken exactly at the position of crossed defect pores (blue) for clarity. We can see that at the distance $\Delta X=2 \mu \mathrm{m}$ away from the crossing pores the spectrum (green in Figure 6.15) is flat in the range of calculated band gap frequencies (gray bar in the Figure). When approaching closer to the crossing pores at the distance $\Delta X=1 \mu \mathrm{m}$ from the cavity position a trough centered at $5955 \mathrm{~cm}^{-1}$ starts to appear in the range of the expected band gap. The through reaches the maximum depth of $22 \%$ of amplitude of the reflectivity peak exactly 


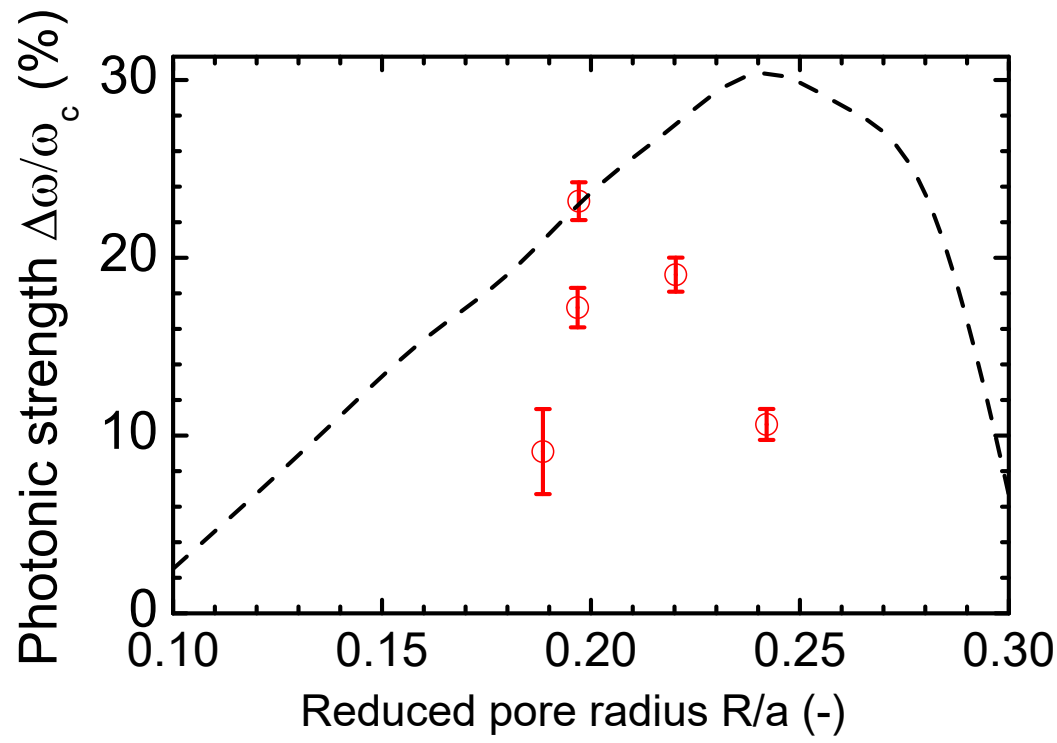

Figure 6.14: $\mathrm{P}$ hotonic strength $\left(\Delta \omega / \omega_{c}\right)$ calculated from band diagram (dashed line) and experimentally observed from reflectivity spectra (red scatterers) as a function of reduced pore radii of the inverse woodpile photonic crystal structure.

at the position where two defect pores crosses (blue spectrum in Fibure 6.15). At the distance $\Delta X=-1 \mu \mathrm{m}$ from the crossing point the trough amplitude slightly decreases to $19.2 \%$ of the stop band peak amplitude. Reflectivity scan at the distance $\Delta X=-2 \mu \mathrm{m}$ from the cavity position exhibits flat spectrum in the range of the calculated band gap alike to the spectrum at $\Delta X=+2 \mu \mathrm{m}$ from the cavity position but with lower amplitude. The decrease in the reflectivity amplitude is associated with the end of photonic crystal. As is clear from the Figure 6.15 the central position of the laser beam during the scan at $\Delta X=-2 \mu \mathrm{m}$ from the cavity is close to the edge of photonic crystal structure and thus considering $4 \mu \mathrm{m}$ size of the laser beam we probe bulk silicon as well as few last unit cells of photonic crystal. Figure 6.16 shows reflectivity spectra measured at different $\Delta Y$ distances around the crossing of two defect pores positions which is indicated with yellow circle centered at the distance $\Delta Y=2.3 \mu \mathrm{m}$ from the edge of the $\mathrm{Si}$ bar. The $Y=0$ is located at the edge of the Si bar since it is easier to determine the edge position from knife-edge measurements than any other position on the bar. The amount of reflected light below the edge of the bar is low since most of the laser beam energy is going into the air but due to the $4 \mu \mathrm{m}$ focus size there is still some intensity on the $\mathrm{Si}$ bar as is seen from the spectra centered at $-0.4 \mu \mathrm{m}$ from the edge of the crystal. Reflectivity spectra at $\Delta Y=0.7 \mu \mathrm{m}$ and $\Delta Y=1.3 \mu \mathrm{m}$ distances from the edge are already close to the cavity located at $\Delta Y=2.3 \mu \mathrm{m}$ from the edge and thus a through appears centered at 5955 $\mathrm{cm}^{-1}$ in the range of calculated band gap. The depth of the through is changing 


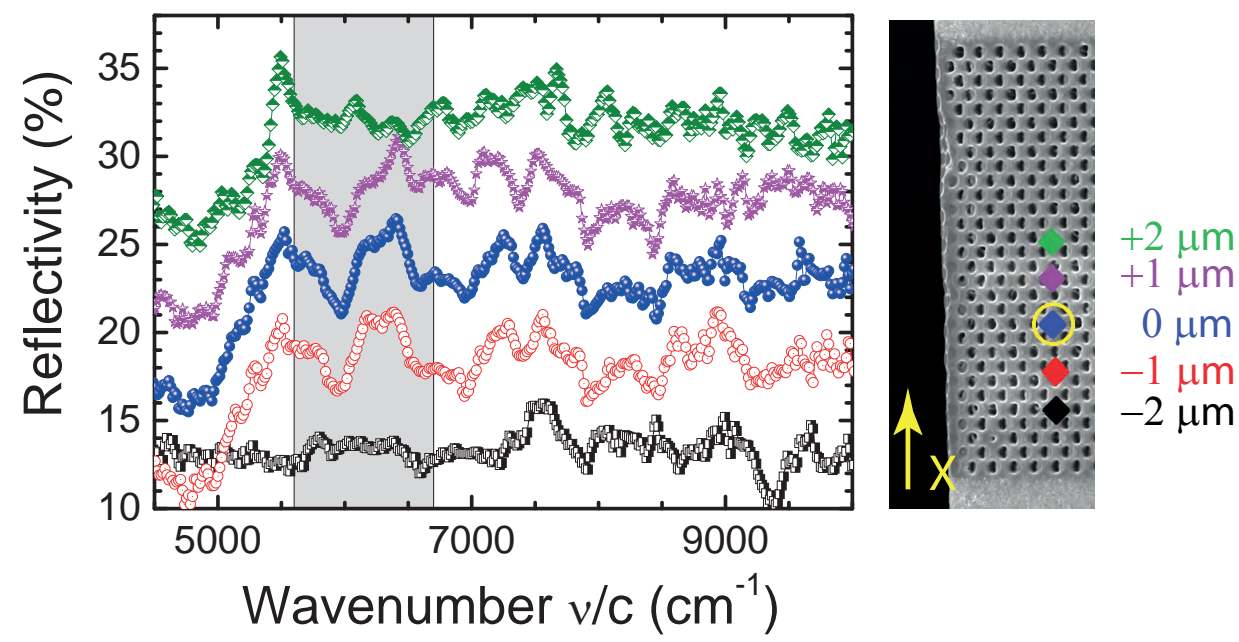

Figure 6.15: Reflectivity spectra taken on the Structure $\mathbf{E}_{\mathbf{1}}$ along the $X$ axis. The $X$ distance is measured from the position of the cavity where two defect pores cross. The through in the reflectivity spectra in the area of the predicted band gap appears when the laser beam approaches the cavity and disappears after the beam is receding from the cavity. The spectra are separated in amplitude by applying a $10 \%$ bias.

from $45 \%$ of the peak amplitude at the distance $0.7 \mu \mathrm{m}$ from the edge to $39 \%$ at the distance $1.3 \mu \mathrm{m}$ from the edge. At the $\Delta Y=2.1 \mu \mathrm{m}$ from the edge the spectrum changes its shape drastically with the significant asymmetry of the stop band peak which makes it difficult to estimate the depth of the trough. Moving further away from the cavity position to $\Delta Y=2.8 \mu \mathrm{m}$ and $\Delta Y=3.5 \mu \mathrm{m}$ from the edge (blue and black spectra in Figure 6.16) makes spectrum flatten in the range of the calculated stop band as is expected. The presence of the trough in the range of calculated band gap frequencies at the spatial positions close to the crossing of two defect pores is and indication of the cavity resonances present in the band gap. The differences between experimental observation of the single trough within the stop band and predicted in the References [15] 5 isolated dispersionless resonances in the band gap can be explained due to first of all differences in the structure design since in the calculations the supercell model was used thus the band structure was calculated for the infinite crystal with two defect pores crossing every $5 \times 5 \times 5$ unit cells. Secondly the crystal under investigation is a finite size structure surrounded by the high refractive index medium $(\mathrm{Si})$ and low refractive index medium (air)

Next we study Structure $\mathbf{D}_{\mathbf{3}}$ (see Appendix B) that consists of the array of 80 cavities arranged in a cubic lattice as shown in Figure 6.9 (b). We choose this design of the array in order to compensate for the finite size of the laser beam and in future investigate light "hopping" from one cavity to another as depicted 

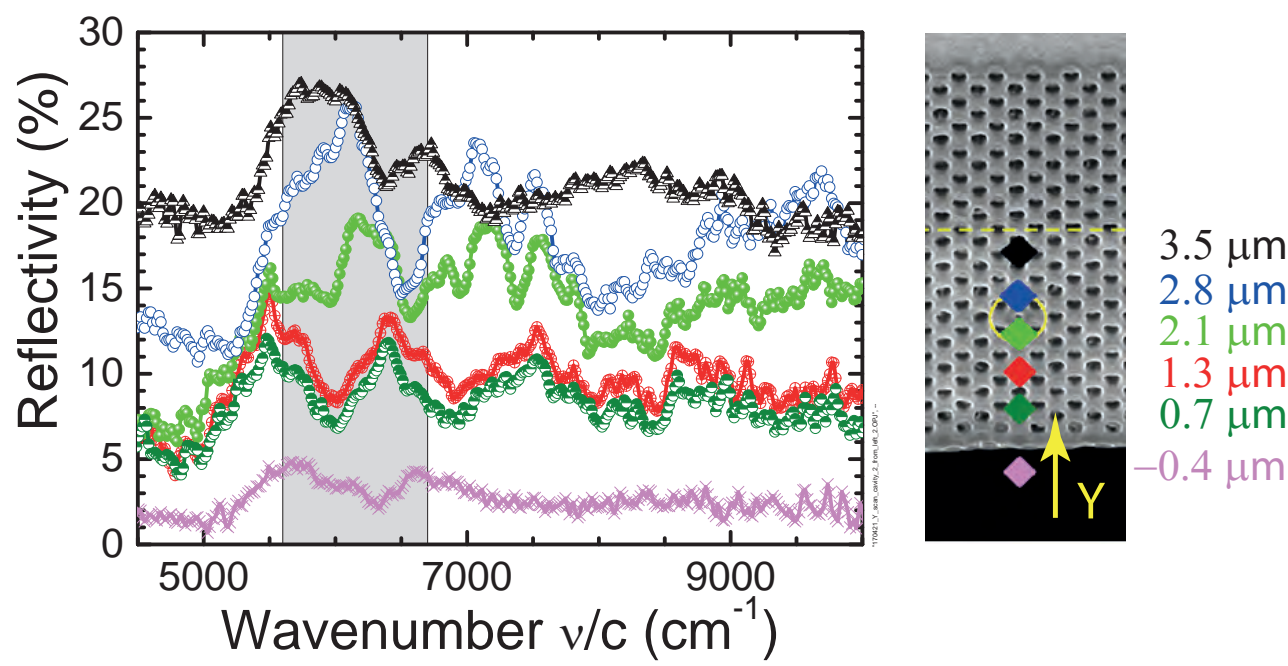

Figure 6.16: Reflectivity spectra taken on the Structure $\mathbf{E}_{\mathbf{1}}$ along the $Y$ axis. The $Y$ distance is measured from the crystal to air interface. The crossing of two defect pores in located at the $2.1 \mu \mathrm{m}$ distance from the crystal to air interface. The through in the reflectivity spectra in the area of the predicted band gap appears when the laser beam reached the crystal surface and disappears after the beam passes the cavity position. The amplitude of each spectrum is unbiased.

in the Chapter 1 Figure 1.1. Moreover the ongoing work of S.A. Hack et al. is concerned about band structure calculations for such 3D crystal of cavities inside a 3D photonic band gap crystal [18]. Since the laser beam focus is not infinitely small during the measurements we are probing all the area that is illuminated withing the beam size as well as regions that are illuminated with the light scattered from the defect. In our case for the broad band white light laser source from the knife-edge measurements we found 90 to $10 \%$ of the intensity to be within $4 \mu \mathrm{m}$ spot. Thus we arranged cavity defects periodically with the period of 3 unit cells in each direction covering the entire crystal volume. Moreover such design is closer to the model used for the $5 \times 5 \times 5$ and $3 \times 3 \times 3$ supercell calculations in the Reference [15] of an infinite photonic crystal with the cavity defect repeated every 5 or 3 unit cells respectively. The reflectivity measured on a Structure $\mathbf{D}_{\mathbf{3}}$ in comparison to the quasi-prefect photonic crystal Structure $\mathbf{D}_{1}$ is shown in Figure 6.17. It is seen from the figure that in the reflectivity measured from the Structure $\mathbf{D}_{\mathbf{3}}$ that contains cavity array two troughs appear within the range of the stop band (indicated with the gray bar). The troughs are centered at 6365 and $6750 \mathrm{~cm}^{-1}$ with the depth of $39 \%$ and $70 \%$ of the peak value respectively. Ongoing band structure calculations of S.A. Hack et al. [18] report bands in this range of frequencies hence sensible that reflectivity is lower. The experimental $Q$ factor of the resonances can be estimated from 


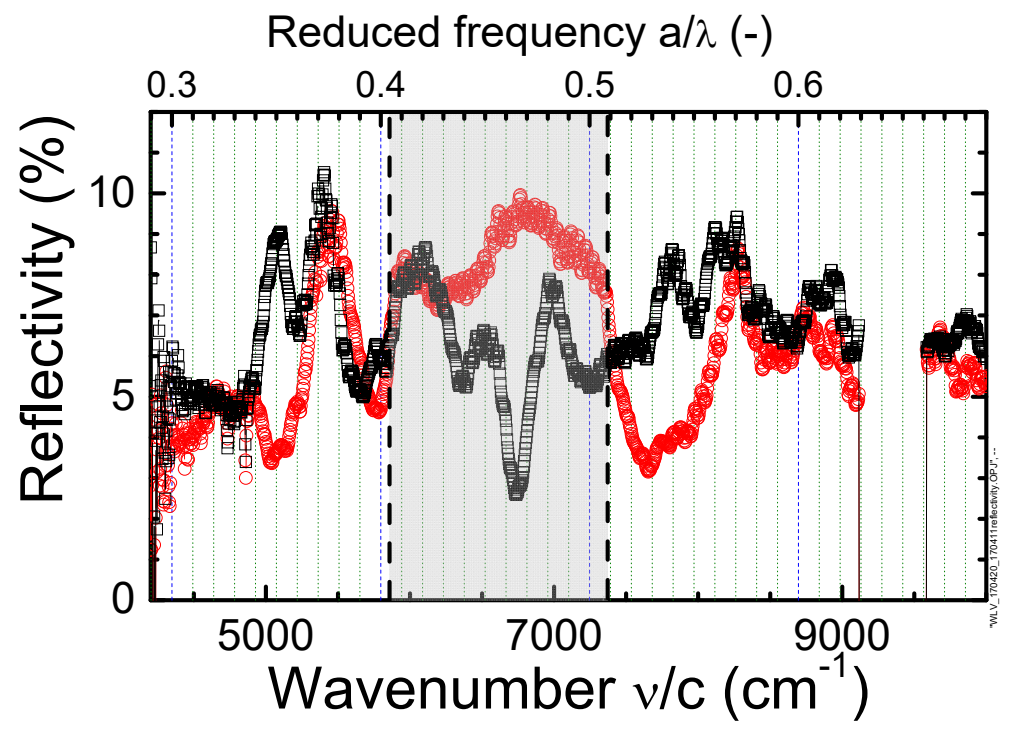

Figure 6.17: Comparison of the reflectivity spectra taken on the quasi-prefect photonic crystal $\mathbf{D}_{\mathbf{1}}$ with no intended defects (red) and photonic crystal Structure $\mathbf{D}_{\mathbf{3}}$ with the array of cavities (black). The stop gap is indicated with the gray bar. The band gap in the spectrum around $9339 \mathrm{~cm}^{-1}$ is filter out to remove artifacts from the pump source of the supercontinuum source.

the ratio $Q_{\text {exp }}=\frac{\lambda}{\Delta \lambda}$ and for the first trough centered at $6365 \mathrm{~cm}^{-1}$ is found to be $Q_{1}=44.8$ and for the second trough centered at $6750 \mathrm{~cm}^{-1}$ is found to be $Q_{2}=40$. The small value of the experimental $\mathrm{Q}$ factors for the resonances in the stop band can be associated with the presence of multiple resonances close to each other in the band gap that can not be resolved in the experiment due to the limited resolution of the spectrometer $8 \mathrm{~cm}^{-1}$ and as well due to the light leaking through the defect pores and scattering.

\subsection{Summary}

We studied optical properties of $2 \mathrm{D}$ and $2 \mathrm{D}$ photonic crystals by means of broadband microscope reflectivity measurements. We described the setup that was build for reflectivity measurements of photonic structures. We investigated in details polarization behavior of light reflected from the $2 \mathrm{D}$ photonic crystals with different configuration of polarizer and analyser. We observed for the first time a large birefringence within the stop bands of $2 \mathrm{D}$ photonic crystals. We interpret polarization behavior of $2 \mathrm{D}$ photonic crystals with the model of semi-infinite birefringent slab with the effective refractive index tensor and obtained the components of the effective refractive index by fitting the model to the experimental 
data. We obtained from the fit model anisotropy of the effective refractive index for frequency range between 4000 and $12000 \mathrm{~cm}^{-1}$. We studied reflectivity measurements of 3D photonic crystal structures with and without embedded defects. From the observed stop bands for quasi-perfect photonic crystal structures we obtained $R / a$ ratio and found an excellent match to the $R / a$ ratios obtained from tomography data. In the reflectivity spectra taken on the structures with embedded defects we observed a trough within the calculated band gap. We found good correspondence of the trough in the reflectivity peak with the spatial position of the cavity defect in $X$ and $Y$ directions. We observed decreased reflectivity in range of predicted extra bands as well as two resonances within the stop band of the crystal containing an array of 80 cavities arranged in a cubic lattice. 


\section{Bibliography}

[1] S. Takayama, H. Kitagawa, Y. Tanaka, T. Asano, and S. Noda, Experimental demonstration of complete photonic band gap in two-dimensional photonic crystal slabs Appl. Phys. Lett. 87, 061107 (2005) 109

[2] A. F. Koenderink, L. Bechger, A. Lagendijk, and W. L. Vos, An experimental study of strongly modified emission in inverse opal photonic crystals Phys. Stat. Sol. (A) 197, (2003) 648-661 109

[3] P. Lodahl, A. F. van Driel, I. S. Nikolaev, A. Irman, K. Overgaag, D. Vanmaekelbergh, and W. L. Vos, Controlling the dynamics of spontaneous emission from quantum dots by photonic crystals Nature 430, (2004) 654-657 109

[4] J.D. Joannopoulos, S.G. Johnson, J.N. Winn, and R. D. Meade, Photonic crystals: molding the flow of light Princeton university press (2008) 110, 115, 118,119

[5] K. M. Ho, C. T. Chan, C. M. Soukoulis, R. Biswas, and M. Sigalas, Photonic band gaps in three dimensions: new layer-by-layer periodic structures Solid State Commun. 89, 413 (1994) 110

[6] C. Marichy, N. Muller, L.S. Froufe-Pe'rez, and F. Scheffold, High-quality photonic crystals with a nearly complete band gap obtained by direct inversion of woodpile templates with titanium dioxide Sci. Rep. 6, 21818: 1-8 (2015) 110

[7] M. J. A. de Dood, B. Gralak, A. Polman, and J.G. Fleming, Superstructure and finite-size effects in a Si photonic woodpile crystal Phys. Rev. B 67, 035322:1-8 (2003) 110

[8] J. Schilling, F. Mller, S. Matthias, R. B. Wehrspohn, and U. Gsele, Threedimensional photonic crystals based on macroporous silicon with modulated pore diameter Appl. Phys. Lett. 78, 1180-1182 (2001) 110

[9] D. Devashish, S.B. Hasan, J.J.W. van der Vegt, and W.L. Vos, Reflectivity calculated for a three-dimensional silicon photonic band gap crystal with finite support Phys. Rev. B 95 (2017) 110

[10] F. Genereux, S.W. Leonard, A. Briner, U. Gösele, and H. M. van Driel, Large birefringence in two-dimensional silicon photonic crystals Phys. Rev. B 63 161101:1-4 (2001) 110, 119

[11] A. Hartsuiker, P. J. Harding, Y. Nowicki-Bringuier, J. M. Grard, and W. L. Vos, Kerr and free carrier ultrafast all-optical switching of GaAs/AlAs nanostructures near the three photon edge of GaAs J. Appl. Phys. 104, 083105: 1-7 (2008) 111

[12] L. A. Woldering, A. P. Mosk, R. W. Tjerkstra, and W. L. Vos, The influence of fabrication deviations on the photonic band gap of three-dimensional inverse woodpile nanostructures J. Appl. Phys. 105, 09310: 1-10 (2009) 112, 121 
[13] S. R. Huisman, R. V. Nair, A. Hartsuiker, L. A. Woldering, A. P. Mosk, and W. L. Vos, Observation of sub-Bragg diffraction of waves in crystals Phys. Rev. Lett. 108, 083901: 1-5 (2012) 112, 113, 120

[14] K.M. Ho, C.T. Chan, and C.M. Soukoulis, Existence of a photonic gap in periodic dielectric structures Phys. Rev. Lett. 25, 3152-3155 (1990) 120

[15] L. A. Woldering, A. P. Mosk, and W. L. Vos, Design of a $3 D$ photonic band gap cavity in a diamond-like inverse woodpile photonic crystal Phys. Rev. B 90, 115140: 1-9 (2014) 125, 127, 128

[16] S. R. Huisman, R. V. Nair, L. A. Woldering, M. D. Leistikow, A. P. Mosk, and W. L. Vos, Signature of a three-dimensional photonic band gap observed on silicon inverse woodpile photonic crystals Phys. Rev. B 83, 205313: 1-7 (2011) $110,122,123$

[17] M. Born and E. Wolf, Principles of Optics Pergamon Press, Oxford, (1970) 115

[18] S.A. Hack, J.J.W. van der Vegt, and W.L.Vos 3D photonic crystal of cavities in a $3 D$ photonic band gap crystal unpublished (2017) 128 



\section{CHAPTER 7}

\section{Outlook}

This thesis presents a proposal and proof-of-concept experiments for a CMOScompatible platform for the realization of 3D silicon nanophotonics. In addition an original x-ray tomography analysis is presented and the optical properties of fabricated structures are studied. In this Chapter we give an outlook for future research in this direction.

To build up a platform for 3D silicon nanophotonics it is important to consider fabrication issues. One of the most common issues with $3 \mathrm{D}$ nanofabrication is so-called overlay - the alignment of features located in different planes or layers. In Chapter 2 of this thesis we already presented a novel method to pattern the etch mask in one step on two oblique surfaces thus with ensured alignment of the features located in different planes. The method that we present allows to fabricate apertures of any arbitrary shape. Hence in future it is interesting to expand the method to more complex mask designs with possible interconnections between oblique surfaces or integrate on-chip devices with different functionalities: for example an optical data detection device on one surface and data processing electronic device in close vicinity on the adjacent surface, or an optical sensing device on one surface and a magneto-electronic memory device on the adjacent surface. The inspiration for the mask designs can be found in opto-electronics, integrated photonics and sensing.

Another direction for development of the single-step etch mask approach for $3 \mathrm{D}$ is to expand it to different types of lithography than reported in Chapter 2. The most promising type of lithography that is widely used nowadays in CMOS industry is deep UV (DUV) lithography. DUV lithography allows to fabricate samples on a wafer scale with high throughput and at low cost per device. The proof-of-principle experiment for exposures of apertures in different depths on the slope of $\mathrm{Si}$ wafer is presented in Chapter 3 of this thesis. The aim of the proofof-principle experiments was to show that it is possible to achieve exposures of the apertures at different depths on the slope within a single DUV exposure. For our test experiments the apertures were located randomly along the slope of a $\mathrm{Si}$ wafer to investigate the positions where the exposures will be successful. The future development of the experiment suggests to design the reticle for the exposure and choose the width and the position of the V-trenches on a wafer based on the previous results to maximize the chance of successful exposure on the slope. Multiple exposures can be used to increase the depth of focus of DUV lithography and hence increase the range of the depths on the slope where apertures can be exposed. The next step in realization of $3 \mathrm{D}$ structures with 
DUV lithography is to transfer pattern from the photoresist either to the hard mask material or directly to silicon. This can be done by means of reactive ion etching (RIE).

Etching of pores in silicon is one of the main processes in current nanofabrication. Developments in the etching equipment allow to constantly improve the process flows to achieve deeper and smoother structures, as for example reported in Chapter 4. To optimize the Bosch process durations of etching and deposition steps, capacitively coupled plasma source and inductively coupled plasma source powers and the gas flows can be tuned. We suggest that further improvements of the etching process can be achieved by investigating in more detail the effect of etching parameters on the resulting structure. For 3D nanofabrication we observe the need in finding an appropriate procedure to clean the polymer residuals from the sample surface that accumulates during the protection steps of Bosch process. Since the etching during the two-step etching process described in this thesis for 3D nanofabrication is not equivalent on the first etch side and second etch side, we also suggest that further investigations are needed to create separate etching process flows for the first and second etch sides. For example CCP and ICP powers can be adjusted for the second etching step taking into account that the hard mask has already undergo one full etching step.

Imaging of 3D nanostructures with X-ray tomography at $10 \mathrm{~nm}$ resolution allows to evaluate fabricated samples and obtain 3D material density distribution. One of the main difficulties during tomography reconstruction that leads to the lower quality of the reconstructed image is the alignment of radiograms taken at different sample-to-detector distances (see Chapter 5). To improve the alignment we propose to design markers of a sharp known shape in both perpendicular surfaces of silicon wafer such that they are visible in transmission radiograms. After the volume of the sample is reconstructed we suggest to expand the analysis of the obtained data to taking cross sections in multiple parallel planes and performing a cross correlation of the images to investigate how much deviations are occurring in the sample with depth. The material density that describes the structure can be used as an input into ab inito numerical models (e.g., FEM or FDTD) to compute optical properties of the structure free from modeling assumptions. Other possible analysis may include the detailed comparison of the material densities inside the pores and outside the sample to obtain if there are residuals of polymer layer in the pores that may hamper subsequent surface chemistry as proposed by A. Schulz.

From the perspective of optical study of the structures described in this thesis we foresee the following interesting future directions. The suggested model to describe large birefringence observed in 2D photonic crystal structures (Chapter 6) can be expanded to include complex refractive indices and non diagonal elements of refractive index tensor. A similar polarization study as was performed on $2 \mathrm{D}$ photonic crystals are performed on 3D photonic crystal structures and need to be analyzed. As a part of "Stirring of light!" program 3D photonic crystals studied in this thesis are suggested to be considered as a back reflector for Si solar cells. Due to the high reflectivity within the band gap for all incident angles and all polarizations it is expected that photonic crystal back reflector will efficiently 
increase absorption of light in Si. A numerical study of the increase in efficiency of solar cells with 3D silicon photonic crystal as a back reflector is currently being carried out by Devashish. Another direction of investigations is to use wavefront to avoid possible disorder and efficiently couple light to a cavity in 3D photonic band gap crystal as studied by R. Uppu. Coupling light to a selected cavity out of an array of cavities will allow to study light "hopping" from one cavity to another and compare to ongoing calculation, as being done by S. Hack. 



\section{APPENDIX A \\ Background on X-ray wave propagation}

\section{A.1 Basic equations.}

Based on Reference [2], in a linear, isotropic, homogeneous and non-dispersive dielectric medium, all components of the electric and the magnetic field behave identically and their behavior is fully described by the following scalar wave equation

$$
\nabla^{2} u(\mathbf{P}, t)-\frac{n^{2}}{c^{2}} \frac{d^{2} u(\mathbf{P}, t)}{d t^{2}}=0,
$$

where $u(\mathbf{P}, t)$ depends on position $\mathbf{P}$ and time $t$, and represents any of the scalar components. The refractive index $n$ of the medium is defined by

$$
n=\left(\frac{\epsilon}{\epsilon_{0}}\right)^{1 / 2}, c=\frac{1}{\sqrt{\mu_{0} \epsilon_{0}}},
$$

where $\epsilon_{\mathbf{0}}$ is the permittivity of vacuum. In the case of x-rays we describe the refractive index of the media as

$$
n=1-\delta+j \beta
$$

where $\delta=r_{c} \lambda^{2} \rho_{e} /(2 \pi)$ and $r_{c}$ is the classical electron radius and $\rho_{e}$ is an electron density. It is convenient to use a Green function approach to solve the inhomogeneous differential equation:

$$
a_{2}(x) \frac{d^{2} U}{d x^{2}}+a_{1}(x) \frac{d^{2} U}{d x}+a_{0}(x) U=V(x) .
$$

We choose here a one-dimensional variable $x$ but equation A.4 can be generalized to a multidimensional position vector $\vec{r}$. When $V(x)$ is replaced by a delta function $\delta\left(x-x^{\prime}\right)$ and with the same boundary conditions the solution of the differential equation is called a Green function $\mathrm{G}(\mathrm{x})$. The general solution $U(x)$ can then be found from

$$
U(x)=\int_{-\infty}^{\infty} G\left(x-x^{\prime}\right) V\left(x^{\prime}\right) d x^{\prime} .
$$

Let us consider the point of observation $P_{0}$ and a closed surface $S$ around it. The problem is to express the optical disturbance at the point $P_{0}$. To solve 
this question we apply the Green function approach and choose as an auxiliary function a unit-amplitude spherical wave expanding from the point $P_{0}$. Thus, the value of $G$ at an arbitrary point $P_{1}$ with vector $\vec{r}$ is given by

$$
G(\vec{r})=\frac{e^{\left(j k r_{01}\right)}}{r_{01}}
$$

To exclude the discontinuity at $P_{0}$, a small spherical surface $S$ of radius $\epsilon$ is inserted around the point $P_{0}$. Green's theorem is then applied, the volume of the integration $\mathrm{V}$ being the volume lying between $S$ and $S_{\epsilon}$ and the surface of integration $S^{\prime}=S+S_{\epsilon}$ ((See Reference [2] 3.18-3.21). From here the integral theorem of Helmholtz and Kirchhoff can be derived, that is used in order to find a field at any point $P_{0}$ (See Reference [2] 3-21):

$$
U\left(P_{0}\right)=\frac{1}{4 \pi} \oint_{s}\left\{\frac{\partial U}{\partial n}\left[\frac{e^{\left(j k r_{01}\right)}}{r_{01}}\right]-U \frac{\partial}{\partial n}\left[\frac{e^{\left(j k r_{01}\right)}}{r_{01}} .\right]\right\} d s
$$

This result is known as the integral theorem of Helmholtz and Kirchhoff and it allows the field at any point $\mathbf{P}_{\mathbf{0}}$ to be expressed in terms of the boundary values of the wave on any closed surface surrounding the point.

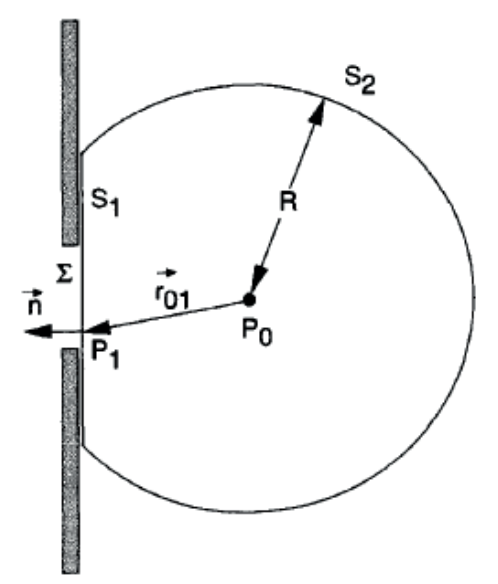

Figure A.1: Kirchhoff diffraction by a plane screen. A wave disturbance impinges from the left on a screen with an aperture and the field is to be calculated at the point $\mathbf{P}_{\mathbf{0}}$. 


\section{A.2 Kirchhoff diffraction}

Let us consider the problem of diffraction of light by an aperture in an infinite opaque screen (A.1) [2]. The wave disturbance impinges on a screen with an aperture from the left and a field at the point $\mathbf{P}_{\mathbf{0}}$ is to be calculated. Having disposed of the integration over the surface $S_{2}$ by taking the limit to infinite radius:

$$
\lim _{R \rightarrow \infty} R\left(\frac{\partial U}{\partial n}-j k U\right)=0
$$

(Goodman $3.4 \mathrm{p} 42$ ), the disturbance at $P_{0}$ is expressed as

$$
U\left(P_{0}\right)=\frac{1}{4 \pi} \iint_{s}\left\{\frac{\partial U}{\partial n} G-U \frac{\partial G}{\partial n}\right\} d s .
$$

Kirchhoff diffraction is considered under the following assumptions: 1 . Across the surface $\Sigma$, the field distribution $U$ and its derivative $\partial U / \partial n$ are exactly the same as they would be in absence of the screen, 2 . Over the portion of $S_{1}$ that lies in the geometrical shadow of the screen, the field distribution $U$ and its derivative $\partial U / \partial n$ are zero.

These conditions are known as Kirchhoff boundary conditions. These assumptions hold in case the dimensions of the aperture $\Sigma$ are large compared to the wavelength $\left(\Sigma \gg \lambda^{2}\right)$. Suppose the aperture to be illuminated by a single spherical wave, $U\left(P_{1}\right)=\frac{\operatorname{Aexp}\left(j k r_{21}\right)}{r_{21}}$ we obtain the Fresnel-Kirchhoff diffraction formula for a field at the point $P_{0}$ : (Goodman 3-25-2-27)

$$
U\left(P_{0}\right)=\frac{A}{j \lambda} \iint_{\Sigma} \frac{\exp \left[j k\left(r_{21}+r_{01}\right)\right]}{r_{21} r_{01}}\left[\frac{\cos \left(\vec{n}, \overrightarrow{r_{01}}\right)-\cos \left(\vec{n}, \overrightarrow{r_{21}}\right)}{2}\right] d s
$$

This equation can be interpreted to imply that the field at the point $P_{0}$ arises from an infinity of fictitious "secondary" point sources located within the aperture, that are related to the illuminating wave front and the angles of illumination and observation.

\section{A.3 Rayleigh-Sommerfeld diffraction}

Kirchhoff theory has an inconsistency since both the normal derivative and the field strength vanish on the boundaries, which mathematically means that function must vanish on the entire plane. Sommerfeld removed this inconsistency by eliminating the necessity for $\boldsymbol{b o t h}$ field strength and its normal derivative to vanish at boundaries. Instead, either field strength or its normal derivative must vanish at the boundaries. Suppose $G$ is generated not only by a point source at $P_{0}$ but also by a source placed at the mirror imaged point $\overline{P_{0}}$ which in case when this two sources oscillate with a $180^{\circ}$ phase difference gives a Green function as

$$
G_{-}\left(P_{1}\right)=\frac{e^{\left(j k r_{01}\right)}}{r_{01}}-\frac{e^{\left(j k \overline{r_{01}}\right)}}{\overline{r_{01}}},
$$


and in case when this two sources are in phase:

$$
G_{+}\left(P_{1}\right)=\frac{e^{\left(j k r_{01}\right)}}{r_{01}}+\frac{e^{\left(j k \overline{r_{01}}\right)}}{\overline{r_{01}}}
$$

which lead to the following Rayleigh-Sommerfeld diffraction formula (Goodman 3-30-3-44):

$$
U_{I}\left(P_{0}\right)=\frac{1}{j \lambda} \iint_{\Sigma} U\left(P_{1}\right) \frac{e^{\left(j k r_{01}\right)}}{r_{01}} \cos \left(\vec{n}, \overrightarrow{r_{01}}\right) d s
$$

In case of $U\left(P_{1}\right)=A \frac{e^{\left(j k r_{21}\right)}}{r_{21}}$ this gives us

$$
U_{I}\left(P_{0}\right)=\frac{A}{j \lambda} \iint_{\Sigma} \frac{e^{\left[j k\left(r_{21}+r_{01}\right)\right]}}{r_{21} r_{01}} \cos \left(\vec{n}, \overrightarrow{r_{01}}\right) d s
$$

and

$$
U_{I I}\left(P_{0}\right)=-\frac{A}{j \lambda} \iint_{\Sigma} \frac{e^{\left[j k\left(r_{21}+r_{01}\right)\right]}}{r_{21} r_{01}} \cos \left(\vec{n}, \overrightarrow{r_{01}}\right) d s
$$

The first Rayleigh-Sommerfeld solution gives a prediction to a well known HuygensFresnel principle: the observed field $U\left(P_{0}\right)$ is a superposition of diverging spherical waves $\exp \left(j k r_{01}\right) / r_{01}$ originating from every point $P_{1}$ withing the aperture $\Sigma$.

From the equation (A.13), the intensity observed on the screen at the distance $r_{01}$ from the aperture is

$$
I_{Q}\left(x_{1}, y_{1}\right)=\left|u_{Q}\right|^{2}=\left|\frac{1}{i \lambda} \iint_{\Sigma} u_{p}(x, y) \frac{e^{\left(j k r_{01}\right)}}{r_{r_{0}}} \cos \left(\vec{n}, \overrightarrow{r_{01}}\right) d s\right|^{2}
$$

In rectangular coordinates the Huygens-Fresnel principle (that comes from the first Rayleigh-Sommerfeld solution) can be expressed as (GM 3-15 and 4-9)

$$
U(x, y)=\frac{z}{j \lambda} \iint_{\Sigma} U(\xi, \eta) \frac{e^{\left(j k\left(r_{01}\right)\right)}}{r_{01}} d \xi d \eta
$$

Where $r_{01}=\sqrt{z^{2}+(z-\xi)^{2}+(y-\eta)^{2}}$.

\section{A.4 Fresnel diffraction}

To reduce Huygens-Fresnel principle, we introduce the following approximation for $r_{01}$ :

$$
r_{01}=z \sqrt{1+\frac{(x-\xi)^{2}}{z^{2}}+\frac{(y-\eta)^{2}}{z^{2}}} \approx z\left[1+\frac{1}{2} \frac{(x-\xi)^{2}}{z^{2}}+\frac{1}{2} \frac{(y-\eta)^{2}}{z^{2}}\right]
$$


The error introduced by dropping all the terms but $z$ in the denominator of equation A.17 is small, however, for $r_{01}$ in the exponent errors are more critical, therefore, equation A.17 turns into

$$
\left.U(x, y)=\frac{e^{(j k z)}}{z j \lambda} \iint_{\Sigma} U(\xi, \eta) e^{\left[j \frac{k}{2 z}\left[(x-\xi)^{2}+(y-\eta)^{2}\right]\right.}\right] d \xi d \eta
$$

and, in another form (see Reference [2], eq. 4-17):

$$
\left.U(x, y)=\frac{e^{(j k z)}}{z j \lambda} e^{\left(j \frac{k}{2 z}\left(x^{2}+y^{2}\right)\right)} \iint_{\Sigma} U(\xi, \eta) e^{\left[j \frac{k}{2 z}\left[\xi^{2}+\eta^{2}\right]\right.}\right] e^{\left(-j \frac{2 \pi}{z \lambda}(x \xi+y \eta)\right.} d \xi d \eta
$$

In order to distinguish the Fresnel and the Fraunhofer regimes the Fresnel number approach is useful: let us define the Fresnel number to be

$$
F \equiv \frac{a^{2}}{L \lambda}
$$

where $a$ is a characteristic size of the aperture, $L$ is the distance between the screen and the aperture. Physically Fresnel number describes the number of zones in which wavefront phase changes by $\pi$ counted from the center to the edge of the aperture as seen from the observational point. If the Fresnel number is less than 1 , then the curvature of the wavefront is small and diffraction follows more simple Fraunhofer description.

\section{A.5 Fraunhofer diffraction}

In the Fraunhofer approximation $\left(z>>\frac{k\left(\xi^{2}+\eta^{2}\right)_{\max }}{2}\right)$, corresponding to a Fresnel number $F<<1$, the field is described as ( Reference [2] equation 4-25)

$$
U(x, y)=\frac{\exp (j k z)}{z j \lambda} \exp \left(j \frac{k}{2 z}\left(x^{2}+y^{2}\right)\right) \int_{-\infty}^{\infty} U(\xi, \eta) \exp \left(-j \frac{2 \pi}{z \lambda}(x \xi+y \eta)\right) d \xi d \eta
$$

Equation A.22 is a Fourier transform of the aperture distribution $U(\xi, \eta)$ multiplied by a phase factor. In the experiment the observable quantity is the intensity which is defined as $I(x, y) \equiv|U(x, y)|^{2}$. This means that in Fraunhofer regime all phase information is lost. Since in x-ray tomography we are interested in phase information, the Fraunhofer regime is not suitable for this purpose.

\section{A.6 Talbot effect for amplitude grating}

Talbot effect is important to consider as a background for x-ray tomography since the presence of a Talbot effect imposes a requirement to record diffraction patterns at multiple sample to detector distances in a tomography experiment. Let 
us first consider a sinusoidal amplitude grating that is modeled as a transmitting structure with an amplitude transmittance equal to

$$
t_{A}(\xi, \eta)=\frac{1}{2}[1+m \cos (2 \pi \xi / L)]
$$

In the Fresnel regime, the transfer function is (GM 4-21)

$$
H(\xi, \eta)=\frac{e^{j k z}}{j \lambda z} e^{\left[j \frac{\pi}{\lambda}\left(\xi^{2}+\eta^{2}\right)\right]}
$$

Therefore, Fourier transform is

$$
F\{H(x, y)\}=e^{\left[-j \pi \lambda z\left(f_{X}^{2}+f_{Y}^{2}\right)\right]}
$$

The frequency spectrum of the field transmitted by the structure is the Fourier transform of the amplitude transmittance:

$$
F\left\{t_{A}(\xi, \eta)\right\}=\frac{1}{2} \delta\left(f_{X}, f_{Y}\right)+\frac{m}{4} \delta\left(f_{X}-\frac{1}{L}, f_{Y}\right)+\frac{m}{4} \delta\left(f_{X}+\frac{1}{L}, f_{Y}\right)
$$

The transfer function in equation (A.25) is equal to 1 at the origin and at $\left(f_{X}, f_{Y}\right)=\left( \pm \frac{1}{L}, 0\right)$

$$
H\left( \pm \frac{1}{L}, 0\right)=e^{\left[-j \frac{\pi \lambda z}{L^{2}}\right]}
$$

Thus after propagation over distance $z$ behind the grating, the Fourier transform of the field becomes

$$
F\{U(x, y)\}=\frac{1}{2} \delta\left(f_{X}, f_{Y}\right)+\frac{m}{4} e^{-j \frac{\pi \lambda z}{L^{2}}} \delta\left(f_{X}-\frac{1}{L}, f_{Y}\right)+\frac{m}{4} e^{-j \frac{\pi \lambda z}{L^{2}}} \delta\left(f_{X}+\frac{1}{L}, f_{Y}\right)
$$

Applying inverse Fourier transform to this spectrum, we find the field in real space at the distance $z$ from the grating to be

$$
U(x, y)=\frac{1}{2}+\frac{m}{4} e^{-j \frac{\pi \lambda z}{L^{2}}} e^{j \frac{2 \pi x}{L}}+\frac{m}{4} e^{-j \frac{\pi \lambda z}{L^{2}}} e^{j \frac{2 \pi x}{L}}=\frac{1}{2}\left[1+m e^{-j \frac{\pi \lambda z}{L^{2}}} \cos \left(\frac{2 \pi x}{L}\right)\right]
$$

Then the intensity distribution $\left(I(x, y)=|U(x, y)|^{2}\right)$ is given by

$$
I(x, y)=\frac{1}{4}\left[1+2 m \cos \left(\frac{\pi \lambda z}{L^{2}}\right) \cos \left(\frac{2 \pi x}{L}\right)+m^{2} \cos ^{2}\left(\frac{2 \pi x}{L}\right)\right]
$$

If we consider the intensity distributions at the so-called Talbot distances $(z=$ $\frac{2 n L^{2}}{\lambda}$, or $\left.\frac{\pi \lambda z}{L^{2}}=2 n \pi\right)$, it is seen that the intensity distributions in such cases are either exact images of the grating, or phase-reversed images (Figure A.2):

1. If $\frac{\pi \lambda z}{L 2}=2 \pi n$, then the intensity is equal to

$$
I(x, y)=\frac{1}{4}\left[1+m \cos \left(\frac{2 \pi x}{L}\right)\right]^{2}
$$

which can be interpreted as a perfect image of the grating. 
2.If $\frac{\pi \lambda z}{L 2}=(2 n+1) \pi$, then the intensity is equal to

$$
I(x, y)=\frac{1}{4}\left[1-m \cos \left(\frac{2 \pi x}{L}\right)\right]^{2}
$$

which corresponds to a perfect image of the grating but with a $180^{\circ}$ spatial phase shift, in other words, a phase-reversed Talbot image.

3.If $\frac{\pi \lambda z}{L 2}=(2 n-1) \pi / 2$, then the intensity is equal to

$$
I(x, y)=\frac{1}{4}\left[1-m^{2} \cos ^{2}\left(\frac{2 \pi x}{L}\right)\right] .
$$

In in this case the image has a doubled frequency compared to the original one and a reduced contrast. This situation is a so-called subimage.

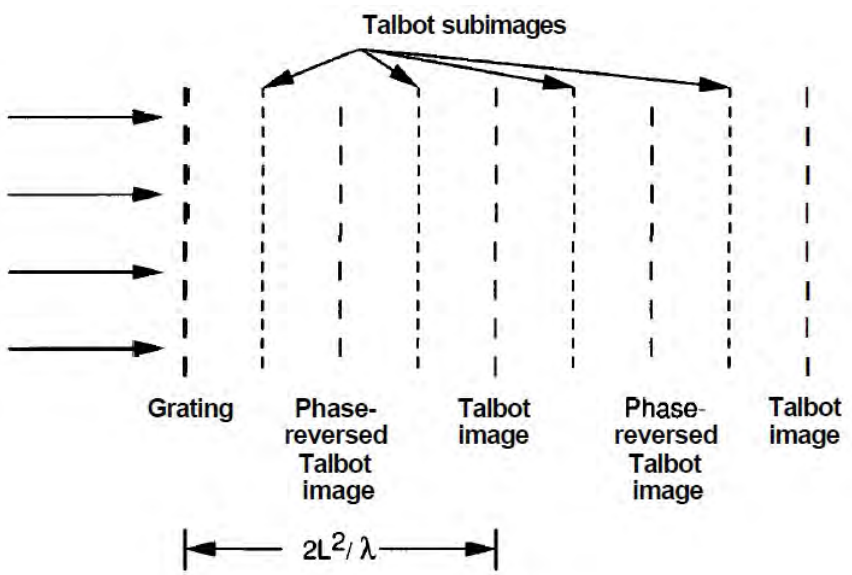

Figure A.2: Spatial location of Talbot images when a sinusoidal amplitude grating grating is illuminated with a plane wave.

\section{A.7 Talbot effect for a phase grating}

In a holotomography experiment that we performed the contrast is provided by the phase delay of the beam propagated through the sample rather than amplitude change, therefore it is important to consider a Fresnel diffraction on a phase periodic sample. Due to Talbot effect it appears to be necessary to perform tomography scans for several distances in order to avoid blindness of Fresnel diffraction to a certain spatial frequencies st every distance.

\section{A.7.1 Numerical calculation in the Fresnel regime.}

In Reference[4] the deep Fresnel diffraction $(r>>\lambda / 2 \pi)$ of a sinusoidal phase grating is considered. The "deep Fresnel regime" is defined as a regime in between 
near-field diffraction, when the propagation distance is confined to a few wavelengths from the grating and far-field Fraunhofer regime. Let the transmission function of the grating $t\left(x_{0}\right)$ be

$$
t\left(x_{0}\right)=\exp \left[j n k a_{0} \sin \left(2 \pi x_{0} / d\right)+j \varphi_{0}\right],
$$

where $k$ is the wavenumber, $a_{0}$ and $d$ thickness and period of the grating respectively, $\varphi_{0}$ is an additional constant phase and $n$ is the refractive index of grating.

The transmission function can be rewritten in terms of Bessel functions of the first kind using the equality

$$
\exp \left[j \frac{m}{2} \sin \left(2 \pi f_{0} \xi\right)\right]=\sum_{q=-\infty}^{\infty} J_{q} \frac{m}{2} \exp \left(j 2 \pi q f_{0} \xi\right)
$$

which gives

$$
t\left(x_{0}\right)=\sum_{m=-\infty}^{\infty} J_{m}\left(n k a_{0}\right) e^{\left[\frac{j 2 \pi m x_{0}}{d}\right]},
$$

The diffraction field $u(x, z)$ in the observation plane satisfies the Helmholtz equation[3] $\left(\nabla+k^{2}\right) u(x, z)=0$, and, using the Green's theorem, the field in the observation plane $u(x, z)$ can be written as

$$
u(x, z)=\frac{1}{4 \pi} \int\left\{u_{0}\left(x_{0}\right) \frac{\partial G\left(x_{0}, x, z\right)}{\partial n_{0}}-G\left(x_{0}, x, z\right) \frac{\partial u_{0}\left(x_{0}\right)}{\partial n_{0}}\right\} d x_{0},
$$

where $G\left(x_{0}, x, z\right)$ is the Green function, $u_{0}\left(x_{0}\right)=A t\left(x_{0}\right)$ is the field immediately after the grating and $\partial / \partial n_{0}$ is the normal derivative. In the one-dimensional case the Green function is $G\left(x_{0}, x, z\right)=j \pi H_{0}^{(1)}(k r)$, where $H_{0}^{(1)}$ is the zero-order Hankel function of the first kind

$$
H_{n}^{(1)}(z)=\frac{1}{j \pi} \int_{0}^{\infty} \frac{e^{(z / 2(t-1 / t))}}{t^{n+1}} d t
$$

and $r=\left[\left(x-x_{0}\right)^{2}+(z-h)^{2}\right]^{1 / 2}$ is the distance from the object point $x_{0}$ to the observational point $x$. Using the recurrence relation for the Hankel function[7] $\left(\left(\frac{1}{z} \frac{d}{d z}\right)^{m}\left(z^{-n} f_{n}(z)\right)=(-1)^{m} z^{-n-m} f_{n+m}(z)\right)$, the derivative of the Green function can be written as

$$
\frac{\partial G\left(x_{0}, x, z\right)}{\partial n_{0}}=\frac{-j \pi k(z-h) H_{1}^{(1)}(k r)}{r} .
$$

When the propagation distance $z$ is larger than several wavelength and the thickness $a_{0}$ is much smaller than the period of the grating, the derivative of the height function $h\left(x_{0}\right)=a_{0} \sin \left(2 \pi x_{0} / d\right)$ is so small compared to the derivative of the Green function that it can be neglected. Therefore the term $\partial u_{0}\left(x_{0}\right) / \partial n_{0}$ in equation A.37 can be neglected as well. Therefore, the diffraction field can be written

$$
u(r)=\frac{-j k r}{4} \int\left[t\left(x_{0}\right)(z-h) H_{1}^{(1)}(k r) / r\right] d x_{0} .
$$


In the deep Fresnel regime $k r>>1$, so that the Hankel function can be replaced by its asymptotic expression $H_{1}^{(1)}(k r) \sim \sqrt{2 / \pi k r} \exp (j k r-j 2 \pi / 4)$. From the equations above, the diffraction intensity of the one-dimensional sinusoidal grating is

$$
I(x, y)=\frac{k A^{2}}{8 \pi}\left|\int \sum_{m=-\infty}^{\infty} J_{m}\left(n k a_{0}\right) e^{\left(j m 2 \pi \frac{x_{0}}{d}\right)} \times(z-h) e^{(i k r)} / r^{3 / 2} d x_{0}\right|^{2}
$$

A numerical calculation of this integral was made with the following parameters: grating size $s=3 \mathrm{~cm}$, wavelength $\lambda=0.8 \mu \mathrm{m}, n=1.532$, grating period $d=20 \mu \mathrm{m}$, grating thickness $a_{0}=1 \mu \mathrm{m}$. Figure A.3 shows a cross section at $y=0$ of the intensity distribution behind the sinusoidal phase grating illuminated with a plane wave. The wave is incident from the left side of the picture on a grating (at $z=0$ ) and propagates to the right side. The interference pattern has a strong dependence on the distance from the grating.

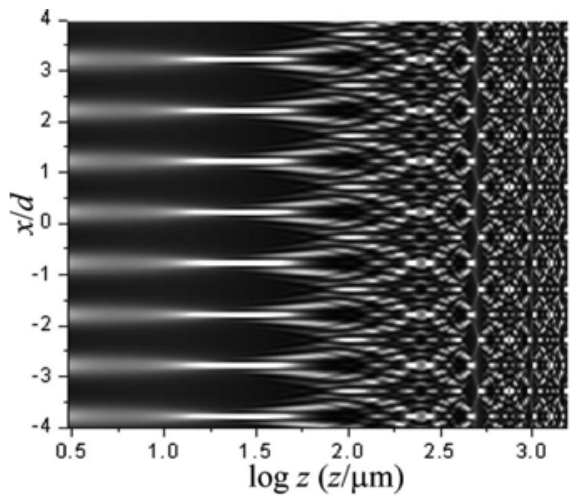

Figure A.3: Diffraction pattern of the sine phase grating in the deep Fresnel region, calculated in Reference[4]. Waves are propagating from left to right. The diffraction pattern shown in the figure is a cross section at the fixed value of $y=0$.

\section{A.7.2 Diffraction using transfer function approach}

Let us now consider a sinusoidal phase grating similar to the 2D photonic crystal structures that surround our 3D crystal which is defined by a transmittance function

$$
t_{A}(\xi, \eta)=\exp \left[j \frac{m}{2} \sin \left(2 \pi f_{0} \xi\right)\right] \operatorname{rect}\left(\frac{\xi}{2 w}\right) \operatorname{rect}\left(\frac{\eta}{2 w}\right),
$$

Where $f_{0}=1 / L$ spatial frequency if the grating, $w$ is a half width of the grating and rect is a rectangular function which has a value of unit when the argument is within $\pm 1 / 2$ interval. To simplify the analysis let us use the identity A.35, where $J_{q}$ is a Bessel function of the first kind, and order q. Given

$$
F\left\{\exp \left[j \frac{m}{2} \sin \left(2 \pi f_{0} \xi\right)\right]\right\}=\sum_{q=-\infty}^{\infty} J_{q} \frac{m}{2} \delta\left(f_{X}-q f_{0}, f_{Y}\right)
$$


A Fourier transform of a transmittance function in that case will be

$$
\begin{aligned}
F & \left\{t_{A}(\xi, \eta)\right\}=\left[A \operatorname{sinc}\left(2 w f_{X}\right) \operatorname{sinc}\left(2 w f_{Y}\right)\right] \otimes\left[\sum_{q=-\infty}^{\infty} J_{q} \frac{m}{2} \delta\left(f_{X}-q f_{0}, f_{Y}\right)\right] \\
& =\sum_{q=-\infty}^{\infty} A J_{q} \frac{m}{2} \operatorname{sinc}\left[2 w\left(f_{X}-q f_{0}\right)\right] \operatorname{sinc}\left(2 w f_{Y}\right)
\end{aligned}
$$

The transfer function in case of Fresnel diffraction is (GM 4-21)

$$
H\left(f_{X}, f_{Y}\right)=\exp \left[-j \pi \lambda z\left(f_{X}^{2}+f_{Y}^{2}\right)\right]
$$

Therefore, using a transfer function approach (3-74 GM), we multiply equations A.44 and A.45 and find the Fourier transform of a field at the position $\mathrm{z}$ to be

$$
F\{U(x, y)\}=\sum_{q=-\infty}^{\infty} A J_{q} \frac{m}{2} \exp \left[-j \pi \lambda z\left(f_{X}^{2}-f_{Y}^{2}\right)\right] \operatorname{sinc}\left[2 w\left(f_{X}-q f_{0}\right)\right] \operatorname{sinc}\left(2 w f_{Y}\right)
$$

Analytical form of the expression A.46 involves convolution integrals that are difficult to interpret therefore it is more revieling to use numerical methods.

Applying numerically inverse Fourier transform to a in Equation A.46, we can obtain field distribution $U(x, y)$ for different distances from the grating. The intensity behind the grating will be then $I(x, y)=|U(x, y)|^{2}$. We plot intensity distribution behind the grating in the Figure A.4. For comparison with the reference[4] we applied the same parameters as described in section A.7.1. In order to simulate the case of x-ray diffraction on photonic crystal sample,

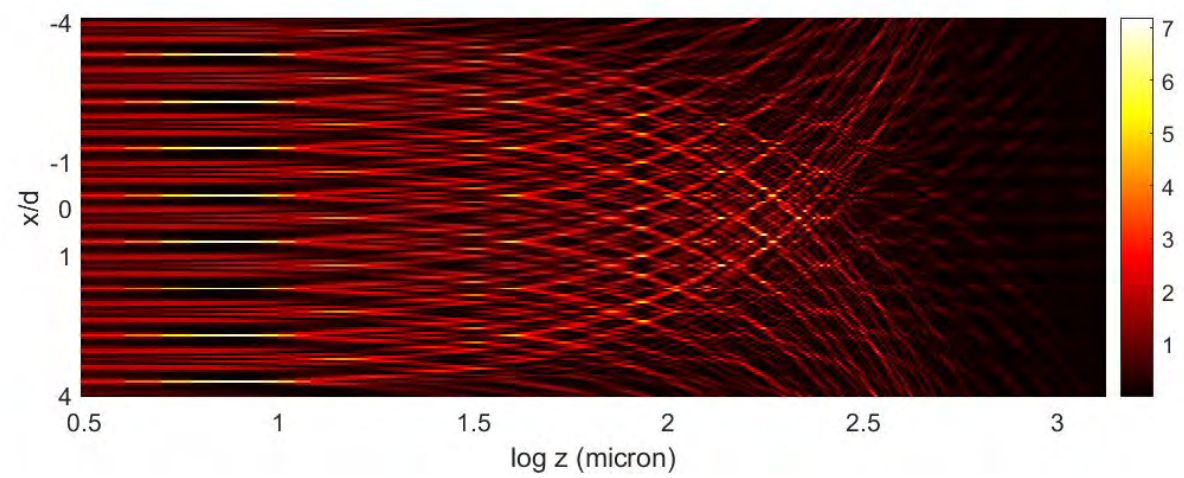

Figure A.4: Diffraction pattern of the sine phase grating with parameters taken from Reference[4] numerically calculated by us using transfer function approach.

the following parameters were chosen: $\lambda=0.072 \mathrm{~nm}$, that corresponds to the beam energy of $17 \mathrm{keV}$; grating period [6] of $690 \mathrm{~nm}$, real part of the refractive index $[5] \delta=1.5$. The diffraction pattern calculated for this case is shown in 
Figure A.5. It is seen that at the number of distances $\mathrm{z}$ such as from the grating there is no contrast. This is a result of Talbot effect for a phase periodic object. The intensity cross sections in Figure A.6 have been taken for com-

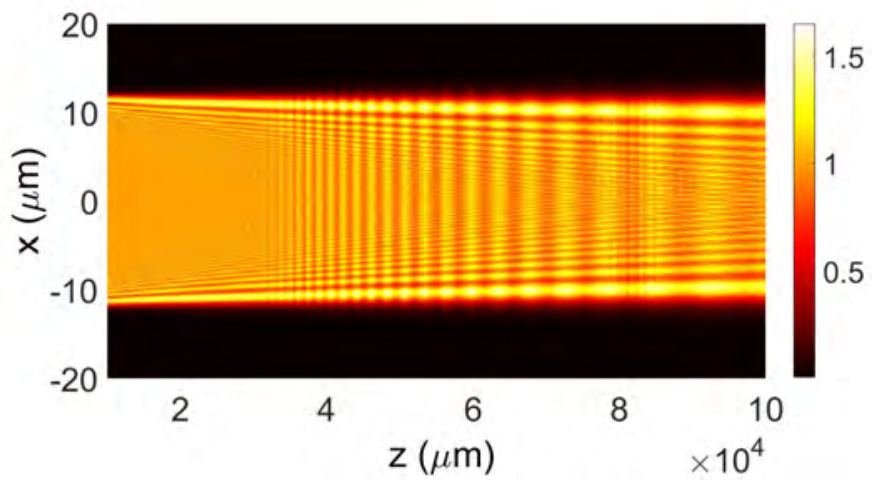

Figure A.5: Diffraction pattern of the sinusoidal phase grating with a period $d=$ $690 \mathrm{~nm}$ illuminated with an x-ray beam with photon energy of $17 \mathrm{keV}$ corresponding to $\lambda=0.07 \mathrm{~nm}$.

parison at the Talbot distances $\left(z_{T b}=N 2 d^{2} / \lambda=130 \mu m\right)$ and at non-Talbot distances $z_{1}=z_{T b}+200 \mu \mathrm{m}$ and $z_{2}=z_{T b}+0.1 \mathrm{~m}$. It is clearly seen that the contrast at Talbot distance is close to zero, unlike the contrast at the distance $z_{1}=z_{T b}+200 \mu \mathrm{m}$. Zero contrast at the Talbot distance for the phase periodic sample means that in order to reconstruct all the spatial frequencies of a periodic sample one need to record diffraction patterns at multiple distances $z$.

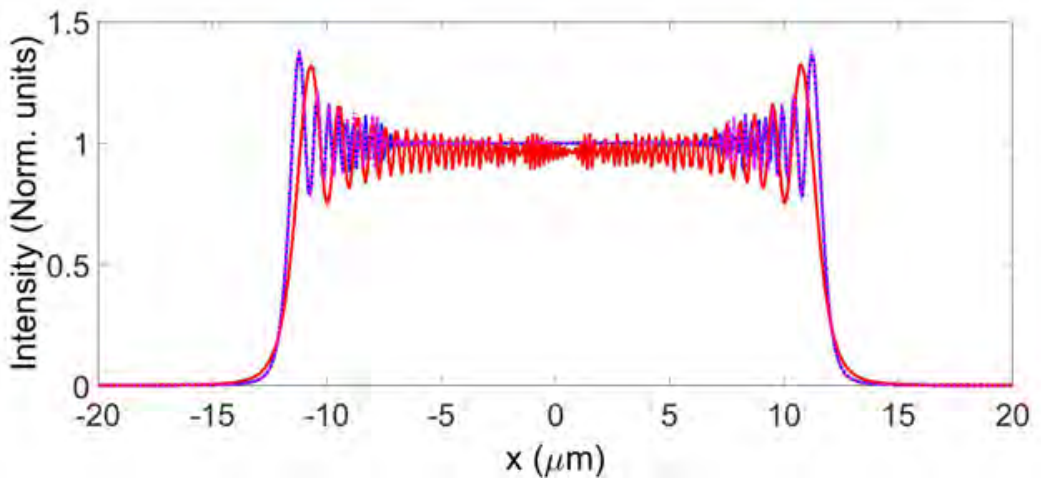

Figure A.6: Cross section of intensity pattern shown in Figure A.5 at the Talbot distance $z_{T b}$ (blue) and for a distances $z_{1}=z_{T b}+200 \mu \mathrm{m}$ (purple) and $z_{2}=z_{T b}+0.1 \mathrm{~m}$ (red). 


\section{A.8 Diffraction pattern from a 2D centered rectangular grating}

Using the same transfer function approach as in the section above we have calculated the intensity distribution for a two-dimensional rectangular centered grating. The grating is chosen to represent the geometry of our 2D photonic crystal. The 2D rectangular centered array of pores is the first step in the fabrication process of our $3 \mathrm{D}$ inverse woodpile photonic crystals[6]. The grating consist of $10 \mu \mathrm{m}$ thick material with real part of the refractive index equal to $\delta=1.5$, which corresponds to refractive index of silicon for $\lambda=0.072 \mathrm{~nm}$. The field distribution $U_{0}$ at the distance $z=0$ just behind grating is shown in the figure A.7. This distribution defines the transmittance of the grating. For calculations we used following grating parameters: $a=2 \mu m, \frac{a}{c}=\sqrt{2}$ and the pore size $d=200 \mathrm{~nm}$. The calculated diffraction intensity distribution over the range of

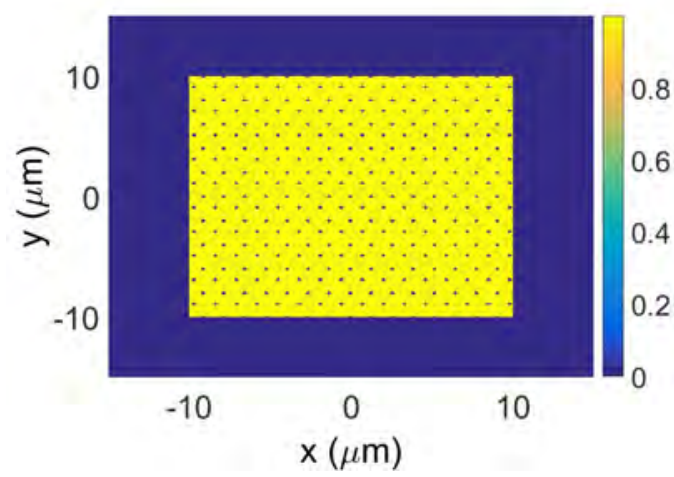

Figure A.7: The field distribution at the distance $z=0$ behind the $2 \mathrm{D}$ centered rectangular grating.

distances for a fixed $y$ value of $y=0.01 \mu \mathrm{m}$ from the center of the grating is shown on the figure A.8. The cross section of intensity distribution for Talbot distance $z_{T b}=5.5 \mathrm{~m}$ and non-Talbot distance $z_{1}=z_{T b}+1 \mathrm{~m}$ is shown in Figure A.9. Notably, that since 2D rectangular centered grating has different periodicity in $x$ and $y$ directions (namely $a$ and $c$ ), the Talbot distances $z_{T b}$ differ for different spacial frequencies of the grating, therefore even at Talbot distances the intensity contrast is non-zero.

In this appendix we described basic theory required for understanding of x-ray interaction with the dielectric media and propagation on the output side. We give the background on such important for x-ray tomography phenomena as Fresnel and Fraunhofer diffraction, Talbot effect. Besides calculations of Talbot effect for an amplitude grating which is widely described in a literature, we provide calculations of Talbot effect for a different types of 2D phase gratings - sinusoidal and centered rectangular grating. 


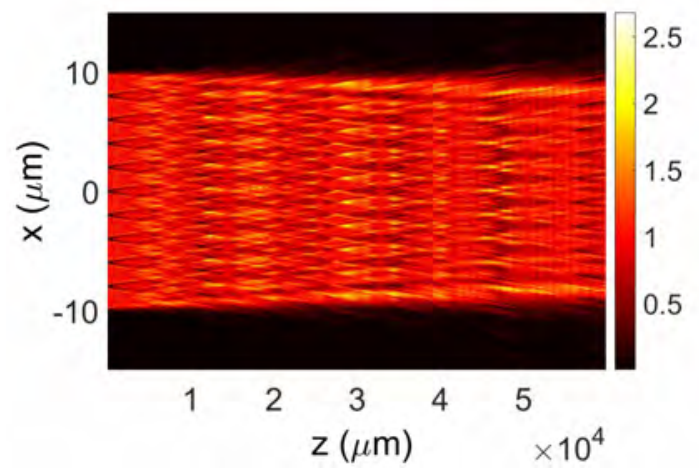

Figure A.8: The diffraction intensity at distance $z$ from the centered rectangular phase grating. The $y$ value is fixed to the $y=0.01 \mu \mathrm{m}$ from the center of the grating.

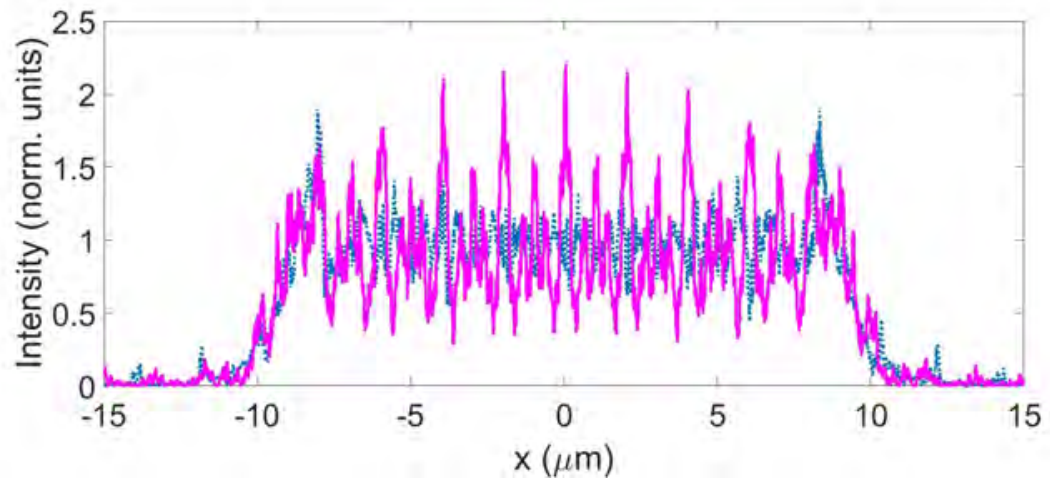

Figure A.9: The cross section of diffraction intensity shown in Figure A.8 at the Talbot distance $z_{T b}=5.5 \mathrm{~m}$ (blue) and at non-Talbot distance $z_{1}=z_{T b}+1 \mathrm{~m}$ (purple). Intensity is normalized to the intensity of incoming wave. 



\section{Bibliography}

[1] C. A. Larabell and M. A. L. Gros, X-ray Tomography Generates 3-D Reconstructions of the Yeast, Saccharomyces cerevisiae, at 60-nm Resolution Mol. Biol. Cell 15, 957-962 (2003)

[2] J.W. Goodman, Introduction to Fourier optics (Roberts and Company, Englewood CO, 2005) 3rd Edition. 139, 140, 141, 143

[3] M. Born, and E. Wolf Principles of Optics, (Cambridge U. Press, 2001) 7th Edition. 146

[4] S. Teng, N. Zhang, Q. Dong, and C. Cheng, Diffraction of a one-dimensional phase grating in the deep Fresnel field J. Opt. Soc. Am. A 24, 3636-3643 (2007) $145,147,148$

[5] M. Deutsch, and M. Hart, X-ray refractive-index measurement in silicon and lithium fluoride, Phys. Rev. B 30, 640-642 (1984) 148

[6] J. M. van den Broek, L. A. Woldering, R. W. Tjerkstra, F. B. Segerink, I. D. Setija, and W. L. Vos Inverse-woodpile photonic band gap crystals with a cubic diamond-like structure made from single-crystalline silicon Adv. Func. Mater. 22, 25-31 (2012) 148, 150

[7] M. Abramowitz and I.A. Stegun Handbook of mathematical functions United States Department of Commerce (1964) 146 



\section{APPENDIX B}

\section{Sample list}

\begin{tabular}{|c|c|c|}
\hline $\begin{array}{l}\text { Sample and } \\
\text { Structure name } \\
\text { in the text }\end{array}$ & $\begin{array}{l}\text { Sample name } \\
\text { in the lab }\end{array}$ & History \\
\hline $\mathbf{A}$ & AD2A24082008 & $\begin{array}{l}\text { 3D photonic crystal sample fabricated with } \\
\text { the two-step etch mask process [1] in Septem- } \\
\text { ber 2008. The 3D structure is surrounded with } \\
\text { a large } 2 \mathrm{D} \text { array of pores. This sample was } \\
\text { kept in a quantum dot suspension for optical } \\
\text { emission measurements reported in [2] subse- } \\
\text { quently rinsed, dried and re-infiltrated several } \\
\text { times. }\end{array}$ \\
\hline B & AD2A2909 & $\begin{array}{l}\text { 3D photonic crystal sample fabricated with } \\
\text { the two-steps etch mask process [1] in August } \\
\text { 2008. The 3D structure is surrounded with a } \\
\text { large 2D array of pores. }\end{array}$ \\
\hline C Structure $\mathbf{1}$ & $\begin{array}{l}\text { PG-10062016- } \\
\text { EBAR-CH02, } \\
\text { Structure 10 }\end{array}$ & $\begin{array}{l}\text { 3D photonic crystal sample fabricated with } \\
\text { the single-step etch mask process described in } \\
\text { the } 2 \text { in June } 2016 \text {. Structure contains a line } \\
\text { defect at the row } 3 \text {. The design } R=150 \\
\text { and } a=750 \text {. 3D structure is surrounded } \\
\text { by bulk silicon. Tomography is performed in } \\
\text { June } 2016 \text {, experiment HC }(2520) \text {. Optical re- } \\
\text { flectivity measurements reported in chapter } 6 \\
\text { were performed in March } 2017\end{array}$ \\
\hline D Structure $\mathbf{1}$ & $\begin{array}{l}\text { PG-EBAR- } \\
\text { 16032017- } \\
\text { CH4B, Struc- } \\
\text { ture } 5\end{array}$ & $\begin{array}{l}\text { 3D photonic crystal sample fabricated with } \\
\text { the single-step etch mask process described in } \\
\text { the } 2 \text { in March } 2017 \text {. Structure is a quasi- } \\
\text { perfect without embedded defects. The design } \\
R=160 \text { and } a=686 \text {. 3D structure is sur- } \\
\text { rounded by bulk silicon. Tomography is per- } \\
\text { formed in April 2017, experiment CH(5092). } \\
\text { Optical reflectivity measurements reported in } \\
\text { chapter } 6 \text { were performed in March } 2017 \text {. }\end{array}$ \\
\hline
\end{tabular}




\begin{tabular}{|c|c|c|}
\hline D Structure $\mathbf{2}$ & $\begin{array}{l}\text { PG-EBAR- } \\
\text { 16032017- } \\
\text { CH4B, Struc- } \\
\text { ture } 12\end{array}$ & $\begin{array}{l}\text { 3D photonic crystal sample fabricated with } \\
\text { the single-step etch mask process described in } \\
\text { the } 2 \text { in March } 2017 \text {. Structure is a quasi- } \\
\text { perfect without embedded defects. The design } \\
R=160 \text { and } a=686 \text {. 3D structure is sur- } \\
\text { rounded by bulk silicon. Tomography is per- } \\
\text { formed in April 2017, experiment CH( } 5092) \text {. } \\
\text { Optical reflectivity measurements reported in } \\
\text { chapter } 6 \text { were performed in March } 2017 \text {. }\end{array}$ \\
\hline D Structure $\mathbf{3}$ & $\begin{array}{l}\text { PG-EBAR- } \\
\text { 16032017- } \\
\text { CH4B, Struc- } \\
\text { ture } 4\end{array}$ & $\begin{array}{l}\text { 3D photonic crystal sample fabricated with } \\
\text { the single-step etch mask process described in } \\
\text { the } 2 \text { in March } 2017 \text {. 3D photonic crystal con- } \\
\text { tains array of cavities. The design } R=160 \\
\text { and } a=686 \text {, defect pore } R^{\prime}=80 \text {. 3D struc- } \\
\text { ture is surrounded by bulk silicon. Tomog- } \\
\text { raphy is performed in April 2017, experiment } \\
\mathrm{CH}(5092) \text {. Optical reflectivity measurements } \\
\text { reported in chapter } 6 \text { were performed in March } \\
2017 \text {. }\end{array}$ \\
\hline E Structure $\mathbf{1}$ & $\begin{array}{l}\text { 3D-Si-bar, } \\
\text { Structure } 4\end{array}$ & $\begin{array}{l}\text { 3D photonic crystal sample fabricated with } \\
\text { the single-step etch mask process described in } \\
\text { the } 2 \text { in October } 2014 \text {. 3D photonic crystal } \\
\text { contains array of cavities. The design } R=160 \\
\text { and } a=686 \text {, defect pore } R^{\prime}=80 \text {. 3D struc- } \\
\text { ture is surrounded by bulk silicon. Optical } \\
\text { reflectivity measurements reported in chapter } \\
6 \text { were performed in October } 2014\end{array}$ \\
\hline
\end{tabular}




\section{Bibliography}

[1] J. M. van den Broek, L. A. Woldering, R. W. Tjerkstra, F. B. Segerink, I. D. Setija, and W. L. Vos, Inverse-woodpile photonic band gap crystals with a cubic diamond-like structure made from single-crystalline silicon, Adv. Func. Mater. 22, 25-31 (2012) 155

[2] M.D. Leistikow, A.P. Mosk, E. Yeganegi, S.R. Huisman, A. Lagendijk, and W.L. Vos, Inhibited spontaneous emission of quantum dots observed in a 3D photonic band gap, Phys. Rev. Lett. 107, 193903 (2011). 



\section{APPENDIX C}

\section{Wave propagation in the birefringent media}

\section{C.1 Wave propagation in the birefringent media}

Let us first consider a uniform plane wave propagating in a lossless isotropic dielectric media [1] in the direction of a wave vector $\mathbf{k}$ given by:

$$
\mathbf{E}(\mathbf{r})=\mathbf{E} \mathbf{e}^{-\mathbf{j k} \cdot \mathbf{r}}, \mathbf{H}(\mathbf{r})=\mathbf{H} \mathbf{e}^{-\mathbf{j k} \cdot \mathbf{r}} \text {, with } \hat{\mathbf{k}} \cdot \mathbf{E}=\mathbf{0}, \mathbf{H}=\frac{\mathbf{n}}{\eta_{\mathbf{0}}} \hat{\mathbf{k}} \times \mathbf{E}
$$

where $n$ is the refractive index of the medium $n=\sqrt{\epsilon / \epsilon_{0}}, \eta_{0}=1 / c_{0} \epsilon_{0}$ the free-space impedance. and $\hat{k}$ the unit-vector in the direction of $\mathbf{k}$, such that

$$
\mathbf{k}=k \hat{k}, k=|\mathbf{k}|=\omega \sqrt{\mu_{0} \epsilon}=n k_{0}, k_{0}=\frac{\omega}{c_{0}}=\omega \sqrt{\mu_{0} \epsilon_{0}}
$$

and $k_{0}$ is the free-space wavenumber. Thus, $E, H, \hat{k}$ form a right-handed system. For the $k$ lying in the xz plane st the angle $\theta$ from z-axis, $\hat{k}=\hat{x} \sin \theta-\hat{z} \cos \theta$, then there are two independent polarization solutions: TM, parallel $(\mathrm{p}=$ polarization $)$ and TE, perpendicular (s-polarization) with fields given by

$$
\begin{aligned}
& \text { TM, p - polarization : } \mathbf{E}=E_{0}(\hat{x} \cos \theta-\hat{z} \sin \theta), \mathbf{H}=\frac{n}{\eta_{0}} E_{0} \hat{y} \\
& \text { TE, } \mathrm{s}-\text { polarization : } \mathbf{E}=E_{0} \hat{y}, \mathbf{H}=\frac{n}{\eta_{0}} E_{0}(-h a t x \cos \theta+\hat{z} \sin \theta)
\end{aligned}
$$

In both cases for TE and TM polarizations the propagation phase factor is

$$
e^{-j k \cdot r}=e^{-j\left(k_{z} z+k_{x} x\right)}=e^{-j k_{0} n(z \cos \theta+x \sin \theta)}
$$

From now on the dielectric interface is taken to be $x y$ plane and the $x z$ plane is a plane of incidence.

Let us now consider a birefringent medium. We choose $x y z$ coordinate axes to coincide with the principal dielectric axes (so that permittivity tensor is diagonal). The wave vector $\mathbf{k}$ lies in the $x z$ plane ant the angle $\theta$ from the $\mathrm{z}$-axis as shown in Figure C.1. The relation between $\mathbf{B}$ and $\mathbf{H}$ is assumed to be $\mathbf{B}=\mu_{0} \mathbf{H}$. Let $\epsilon_{1}, \epsilon_{2}, \epsilon_{3}$ be the permittivity values along the principal axes with corresponding refractive indexes $n_{i}=\sqrt{\epsilon_{i} / \epsilon_{0}}$, where $i=1,2,3$. Then the relationship between $D$ and $E$ is: 

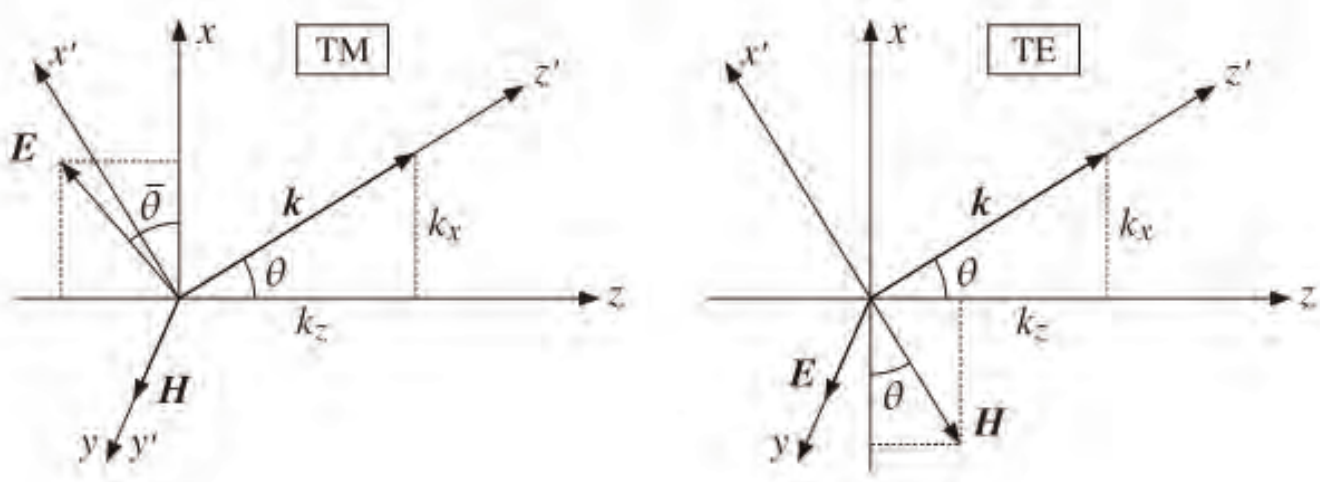

Figure C.1: The geometry of the TE and TM wave. The coordinate system $x y z$ is aligned with principal axis of the dielectric media and the coordinate system $x^{\prime} y^{\prime} z^{\prime}$ is aligned with the wave vector $\mathbf{k}$. The dielectric interface is taken to be $x y$ plane.

values along the principal axes with corresponding refractive indices

$$
\left[\begin{array}{l}
D_{x} \\
D_{y} \\
D_{z}
\end{array}\right]=\left[\begin{array}{ccc}
\epsilon_{1} & 0 & 0 \\
0 & \epsilon_{2} & 0 \\
0 & 0 & \epsilon_{3}
\end{array}\right]\left[\begin{array}{l}
E_{x} \\
E_{y} \\
E_{z}
\end{array}\right]=\epsilon_{0}\left[\begin{array}{ccc}
n_{1}^{2} & 0 & 0 \\
0 & n_{2}^{2} & 0 \\
0 & 0 & n_{3}^{2}
\end{array}\right]\left[\begin{array}{l}
E_{x} \\
E_{y} \\
E_{z}
\end{array}\right]
$$

The wave vector $\mathbf{k}$ can be written as follows:

$$
k=k \hat{k}=k(\hat{x} \sin \theta+\hat{z} \cos \theta)=\hat{x} k_{x}+\hat{z} k_{z}
$$

We now may define the effective refractive index $N$ such that:

$$
k=N k_{0}=N \frac{\omega}{c_{0}}
$$

In order to derive $\mathrm{N}$ let us find propagating solutions for Maxwell equations. Taking into account that gradient of the fields is equal to $-j \mathbf{k}$, Maxwell equations can be written as

$$
\begin{aligned}
& \nabla \times \mathbf{E}=-j \omega \mu_{0} \mathbf{H} \quad k \times \mathbf{E}=\omega \mu_{0} \mathbf{H} \\
& \nabla \times \mathbf{H}=-j \omega \mathbf{D} \quad k \times \mathbf{H}=-\omega \mathbf{D} \\
& \nabla \cdot \mathbf{D}=0 \quad \mathbf{k} \cdot \mathbf{D}=0 \\
& \nabla \cdot \mathbf{H}=0 \quad \mathbf{k} \cdot \mathbf{H}=0
\end{aligned}
$$

Replacing $\mathbf{k}=k \hat{k}=N k_{0} \hat{k}$, where $\mathrm{N}$ is the effective refractive index to be determined.

$$
\begin{aligned}
& N \frac{\omega}{c_{0}} \hat{k} \times \mathbf{E}=\omega \mu_{0} \mathbf{H}, \\
& H=\frac{N}{\eta_{0}} \hat{k} \times E
\end{aligned}
$$


Where $\eta_{0}=c_{0} \mu_{0}$. Then we write from equations C.7:

$$
\begin{aligned}
& \mathbf{D}=-\frac{1}{\omega} \mathbf{k} \times \mathbf{H}=-\frac{1}{\omega} N \frac{\omega}{c_{0}} \hat{k} \times \mathbf{H}=\frac{N^{2}}{\eta_{0} c_{0}} \hat{k} \times(\mathbf{E} \times \hat{k}) \\
& \hat{k} \times(\mathbf{E} \times \hat{k})=\frac{1}{\epsilon_{0} N^{2}} \mathbf{D}
\end{aligned}
$$

Using the vectorial multiplication rules we can transform $\hat{k} \times(\mathbf{E} \times \hat{k})=\mathbf{E}-\hat{k}(\hat{k} \cdot \mathbf{E})$. Thus we obtain

$$
\mathbf{E}=\frac{1}{\epsilon_{0} N^{2}} \mathbf{D}=\hat{k}(\hat{k} \cdot \mathbf{E})
$$

This is a linear equation thus in order to have a non trivial solution its determinant must be zero. This provides a condition to find $N$. Let us write the equation C.10 separately for each component of $\mathbf{E}$. Here we use the expression for $\mathbf{D}$ and that $\hat{k} \cdot \mathbf{E}=E_{x} \sin \theta+E_{z} \cos \theta$ :

$$
\begin{aligned}
& \left(1-\frac{n_{1}^{2}}{N^{2}}\right) E_{x}=\left(E_{x} \sin \theta+E_{z} \cos \theta\right) \sin \theta \\
& \left(1-\frac{n_{2}^{2}}{N^{2}}\right) E_{y}=0 \\
& \left(1-\frac{n_{3}^{2}}{N^{2}}\right) E_{z}=\left(E_{x} \sin \theta+E_{z} \cos \theta\right) \cos \theta
\end{aligned}
$$

Here we consider two cases separately: TE and TM polarizations.

\section{C.1.1 TE polarization}

In case of TE polarization $E_{y} \neq 0$ and $E_{x}=E_{z}=0$. Thus second from the set of equations C.11 immediately implies that $N=n_{2}$. Then we obtain solution for TE case:

$$
\begin{aligned}
& \mathbf{E}(\mathbf{r})=E_{0} \hat{y} e^{(-j \mathbf{k} \cdot \mathbf{r})} \\
& \mathbf{H}(\mathbf{r})=\frac{n_{2}}{\eta_{0}} E_{0}(-\hat{x} \cos \theta+\hat{z} \sin \theta) e^{(-j \mathbf{k} \cdot \mathbf{r})}
\end{aligned}
$$

and the TE propagation factor is

$$
e^{(-j \mathbf{k} \cdot \mathbf{r})}=e^{\left(-j k_{0} n_{2}(z \cos \theta+x \sin \theta)\right)}
$$

\section{C.1.2 TM polarization}

For TM polarization $E_{x} \neq 0, E_{z} \neq 0$ and $E_{y}=0$. Then linear system C.11 becomes

$$
\begin{aligned}
& \left(1-\frac{n_{1}^{2}}{N^{2}}\right) E_{x}=\left(E_{x} \sin \theta+E_{z} \cos \theta\right) \sin \theta \\
& \left(1-\frac{n_{3}^{2}}{N^{2}}\right) E_{z}=\left(E_{x} \sin \theta+E_{z} \cos \theta\right) \cos \theta
\end{aligned}
$$


Using $\sin ^{2} \theta+\cos ^{2} \theta=1$ we can rewrite above equations in a matrix form:

$$
\left[\begin{array}{cc}
\cos ^{2} \theta-\frac{n_{1}^{2}}{N^{2}} & -\sin \theta \cos \theta \\
-\sin \theta \cos \theta & \sin ^{2} \theta-\frac{n_{3}^{2}}{N^{2}}
\end{array}\right]\left[\begin{array}{l}
E_{x} \\
E_{z}
\end{array}\right]=0
$$

To have non trivial solutions the matrix has to have zero determinant, thus

$$
\left(\cos ^{2} \theta-\frac{n_{1}^{2}}{N^{2}}\right)\left(\sin ^{2} \theta-\frac{n_{3}^{2}}{N^{2}}\right)-\sin ^{2} \theta \cos ^{2} \theta=0
$$

Solving the above equation we get

$$
\begin{aligned}
& \frac{1}{N^{2}}=\frac{\cos ^{2} \theta}{n_{1}^{2}}+\frac{\sin ^{2} \theta}{n_{3}^{2}} \\
& N=\frac{n_{1} n_{3}}{\sqrt{n_{1}^{2} \sin ^{2} \theta+n_{3}^{2} \cos ^{2} \theta}}
\end{aligned}
$$

Solving the linear system we find for TM polarization

$$
\begin{aligned}
& \mathbf{E}(\mathbf{r})=E_{0} \frac{N}{\sqrt{n_{1}^{2}+n_{3}^{2}-N^{2}}}\left(\hat{x} \frac{n_{3}}{n_{1}} \cos \theta-\hat{z} \frac{n_{1}}{n_{3}} \sin \theta e^{(-j \mathbf{k r})}\right. \\
& \mathbf{H}(\mathbf{r})=\frac{E_{0}}{\eta_{0}} \frac{n_{1} n_{3}}{\sqrt{n_{1}^{2}+n_{3}^{2}-N^{2}}} \hat{y} e^{(-j \mathbf{k r})}
\end{aligned}
$$

with TM propagation factor

$$
e^{(-j \mathbf{k} \cdot \mathbf{r})}=e^{\left(-j k_{0} N(z \cos \theta+x \sin \theta)\right)}
$$

\section{C.1.3 Reflection and refraction from birefringent medium}

Here we now consider reflection and refraction of waves from the birefringent media. Figure C.2 shows the geometry of TE and TM cases.

The plane wave is incident from both sides on a planar interface separating two media $\epsilon$ and $\epsilon^{\prime}$. In case of TM polarization the electric fields lie on the plane of incidence and magnetic fields are perpendicular to that plane when in TE case it is other way around - electric fields are perpendicular to the plane if incidence and magnetic fields lie in that plane. In case of birefringent media left and right media are described with triplets of principal indices $\mathbf{n}=\left[n_{1}, n_{2}, n_{3}\right]$ and $\mathbf{n}^{\prime}=\left[n_{1}^{\prime}, n_{2}^{\prime}, n_{3}^{\prime}\right]$. The principal indices are aligned along $x y z$ axes, the $x y$-plane is the interface plane, and the $x z$-plane is the plane of incidence. The boundary conditions require that tangential components of electric field are matched, which means $E_{x}$ components in the TM case and $E_{y}$ in the TE case. Thus we can express equations C.12 in terms of $E_{y}$ and equation C.17 in terms of $E_{x}$. Taking into account that for the TM case $\mathbf{E}=\hat{x} E_{x}+\hat{z} E_{z}=E_{x}(\hat{x}-\hat{z} \tan \hat{\theta})$ we obtain

$$
\begin{aligned}
& \mathbf{E}(\mathbf{r})=E_{x}\left(\hat{x}-\hat{z} \frac{n_{1}^{2}}{n_{3}^{2}} \tan \hat{\theta}\right) e^{(-j \mathbf{k} \cdot \mathbf{r})} \\
& \mathbf{H}(\mathbf{r})=\frac{E_{x} n_{1}^{2}}{\eta_{0} N \cos \theta} \hat{y} e^{(-j \mathbf{k} \cdot \mathbf{r})}
\end{aligned}
$$



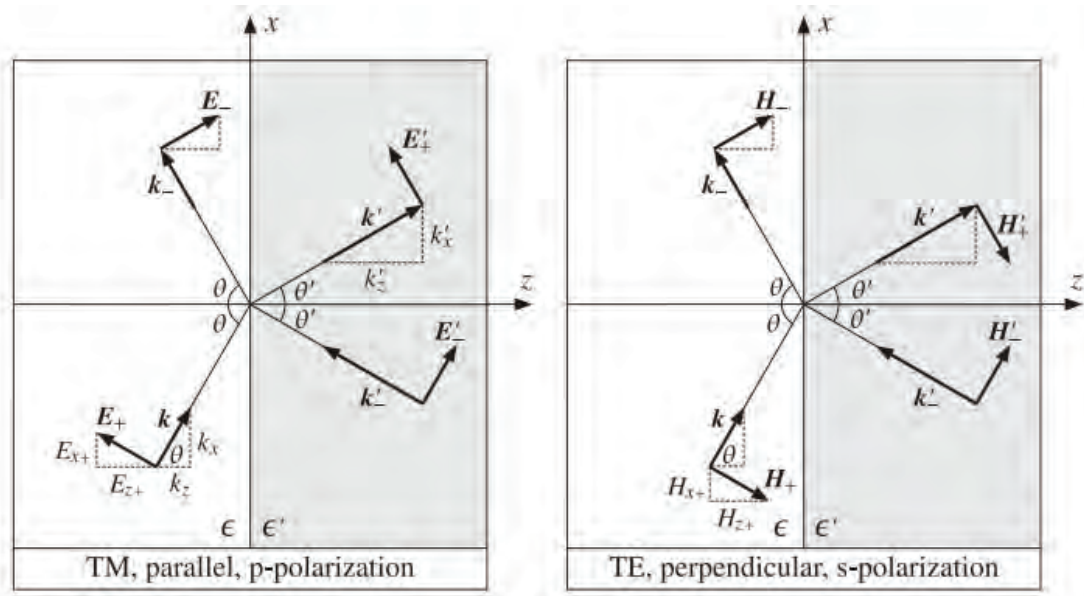

Figure C.2: The geometry of the reflection and refraction for TE and TM wave. The coordinate system $x y z$ is aligned with principal axis of the dielectric media and the coordinate system $x^{\prime} y^{\prime} z^{\prime}$ is aligned with the wave vector $\mathbf{k}$.

values along the principal axes with corresponding refractive indices

Similarly we write for the TE case:

$$
\begin{aligned}
& \mathbf{E}(\mathbf{r})=E_{y} \hat{y} e^{(-j \mathbf{k} \cdot \mathbf{r})} \\
& \mathbf{H}(\mathbf{r})=\frac{E_{y} n_{2} \cos \theta}{\theta_{0}}(-\hat{x}+\hat{z} \tan \theta) e^{(-j \mathbf{k} \cdot \mathbf{r})}
\end{aligned}
$$

Where the propagation factors are:

$$
e^{(-j \mathbf{k} \cdot \mathbf{r})}=e^{\left(-j k_{0} x N \sin \theta-j k_{0} z N \cos \theta\right)}
$$

with the corresponding effective refractive indices $N$. Assuming the interface is at $z=0$, the equality of the total tangential electric fields implies the prapagation phase phactors to match at all values of $x$ :

$$
e^{\left(-j k_{x+} x\right)}=e^{\left(-j k_{x-} x\right)}=e^{\left(-j k_{x+}^{\prime} x\right)}=e^{\left(-j k_{x-}^{\prime} x\right)}
$$

which means that $k_{x+}=k_{x-}=k_{x+}^{\prime}=k_{x-}^{\prime}$ and since $k_{x}=k \sin \theta=N k_{0} \sin \theta$ :

$$
N \sin \theta_{+}=N \sin \theta_{-}=N^{\prime} \sin \theta_{+}^{\prime}=N^{\prime} \sin \theta_{-}^{\prime}
$$

This equation provides us Snel's law for the birefringent media:

$$
N \sin \theta=N^{\prime} \sin \theta
$$

Due to the dependence of $\mathrm{N}$ on polarization, there will be two different refraction angles for the same angle of incidence. The Snel's law can be thus written 
separately for two polarizations:

TM, p - polarization : $\frac{n_{1} n_{3} \sin \theta}{\sqrt{n_{1}^{2} \sin ^{2} \theta+n_{3}^{2} \cos ^{2} \theta}}=\frac{n_{1} n_{3} \sin \theta}{\sqrt{n_{1}^{2} \sin ^{2} \theta+n_{3}^{2} \cos ^{2} \theta}}$

TE, s - polarization : $n_{2} \sin \theta=n_{2}^{\prime} \sin \theta$

The Fresnel coefficients for the TE and TM case are the following:

$$
\begin{aligned}
& \rho_{T M}=\frac{n_{T M}-n_{T M}^{\prime}}{n_{T M}+n_{T M}^{\prime}}=\frac{\frac{n_{1}^{2}}{N \cos \theta}-\frac{n_{1}^{2}}{n_{1}^{\prime} \cos ^{\prime} \theta}}{\frac{n_{1}^{2}}{N \cos \theta}+\frac{n_{1}^{2}}{N^{\prime} \cos ^{\prime} \theta}} \\
& \rho_{T E}=\frac{n_{T E}-n_{T E}^{\prime}}{n_{T E}+n_{T E}^{\prime}}=\frac{n_{2} \cos \theta-n_{2}^{\prime} \cos ^{\prime} \theta}{n_{2} \cos \theta+n_{2}^{\prime} \cos ^{\prime} \theta}
\end{aligned}
$$

Were we used transverse refractive indices

$$
\begin{aligned}
& n_{T M}=\frac{n_{1} n_{3}}{\sqrt{n_{3}^{2}-N^{2} \sin ^{2} \theta}} \\
& n_{T E}=n_{2} \cos \theta
\end{aligned}
$$

We can also write analytical expressions for reflection coefficients in terms of the angle $\theta$ only:

$$
\begin{aligned}
\rho_{T M}= & \frac{n_{1} n_{3} \sqrt{n_{3}^{\prime 2}-N^{2} \sin ^{2} \theta}-n_{1}^{\prime} n_{3}^{\prime} \sqrt{n_{3}^{2}-N^{2} \sin ^{2} \theta}}{n_{1} n_{3} \sqrt{n_{3}^{\prime 2}-N^{2} \sin ^{2} \theta}+n_{1}^{\prime} n_{3}^{\prime} \sqrt{n_{3}^{2}-N^{2} \sin ^{2} \theta}} \\
\rho_{T E}= & \frac{n_{2} \cos \theta-\sqrt{n_{2}^{\prime 2}-n_{2}^{2} \sin ^{2} \theta}}{n_{2} \cos \theta+\sqrt{n_{2}^{\prime 2}-n_{2}^{2} \sin ^{2} \theta}}
\end{aligned}
$$

In Chapter 5 we use the absolute square of the equations C.28 to determine the intensities of TE and TM reflected light. 


\section{Bibliography}

[1] S.J. Orfanidis Electromagnetic Waves and Antennas Available online http://eceweb1.rutgers.edu/ orfanidi/ewa/ (on 170511) 159 



\section{Nederlandse samenvatting}

In deze dissertatie bestuderen en ontwikkelen wij een platform voor het realizeren van driedimensionale (3D) silicium nanofotonica. Het veld van de silicium fotonica heeft zich in al afgelopen 20 jaar gestaag ontwikkeled. Alle benodigde structuren worden tot nu toe gemaakt op planaire wijze in een tweedimensionale vlak. Er wordt moeite gedaan om zulke $2 \mathrm{D}$ structuren te stapelen om zo 3D structuren te vormen waarbij het een grote uitdaging is om 3D structuren monolitisch te maken. In hoofdstuk 2 van deze dissertatie doen we een voorstel voor en demonstreren een enkelstaps etsmasker om 3D nanostructuren te fabriceren door op twee loodrecht op elkaar staande vlakken in een stap een patroon te genereren. Het gewenste patroon is op twee aanliggende vlakken geprojecteerd als een foto zodat de uitlijning van de structuren op de verschillende vlakken is vastgelegd in het ontwerp. Op deze manier ontwijken wij het probleem van uitlijning dat zo centraal is in de huidige halfgeleiderindustrie. We tonen verschillende voorbeelden van etsmaskers gemaakt met de enkelvoudige fotografische methode. De maskers die worden getoond in dit hoofdstuk zijn ontworpen om 3D fotonische kristallen te produceren. We tonen aan dat de uitlijning van openingen in verschillende vlakken binnen 3-5 $\mathrm{nm}$ is, wat hoofdzakelijk bepaald wordt door de nauwkeurigheid van de elektronen microscoop. In hoofdstuk 3 doen we het voorstel en tonen we aan hoe we de voorgenoemde enkelvoudige methode kunnen uitbreiden naar diep Ultraviolet (DUV) lithografie en belichten van openingen op het hellende vlak op een silicium wafel. Het hebben van masker openingen op loodrecht op elkaar staande vlakken is de sleutel tot het bereiken van 3D structuren na het etsen. We presenteren de eerste experimenten op het homogeen bedekken van de hellende vlakken met een laag fotogevoelige lak. We laten zien dat het met een enkelvoudige ultraviolet belichting mogelijk is om openingen te fabriceren op 1.18 micrometer diep op de helling. In hoofdstuk 4 bediscussiëren we het etsen van diepe poriën in silicium. We beschouwen de diep reactieve etsprocesparameters voor het etsen van poriën met extreem grote aspect verhoudingen in silicium. Het proces dat we hebben ontworpen staat ons toe om nanoporiën te etsen met een aspect verhouding van 42 . Hierna beschouwen we het etsen van twee loodrechte sets van poriën in silicium voor het vormen van drie dimensionale structuur en het noodzakelijke bijstellen van de ets parameters. We stellen voor om een extra zuurstofplasma schoonmaak stap toe te voegen tussen het etsen van de twee sets poriën om de achtergebleven polymeer resten van de voorgaande stappen te verwijderen. We fabriceren en presenteren 3D fotonische bandkloof kristallen met en zonder ingebouwde defecten. In hoofdstuk 5 presenteren we de toepassing van zoom tomografie met röntgestraling als niet invasieve beeld vormende techniek 
van 3D fotonische nanostructuren. We beschrijven het experimentele schema en we geven de volledige afleiding van de theoretische beschrijving van het faseherleidingalgoritme dat noodzakelijk is voor de reconstructie van de beelden. We passen zoom tomografie toe op onze 3D fotonische bandkloof kristallen en nemen 3D materiaal dichtheid verdeling in de gefabriceerde kristallen waar met een ultieme resolutie van 20 en $10 \mathrm{~nm}$. Ook presenteren we een binair model dat tomografie data beschrijft en het toestaat om deze te gebruiken als invoer voor $a b$ initio numerieke modellen met als doel om de optische eigenschappen vrije vorm aannames te berekenen. In hoofdstuk 6 bestuderen we de optische eigenschappen van $2 \mathrm{D}$ en $3 \mathrm{D}$ fotonische kristallen door middel van breedband microscopische reflectiviteit metingen. We onderzoeken het polarisatie gedrag van licht dat gereflecteerd wordt door 2D fotonische kristallen met verschillende configuraties van de polarisator en analysator en nemen voor het eerst een grote mate van dubbelbreking binnen de stop band waar. We interpreteren het polarisatie gedrag van het $2 \mathrm{D}$ kristal met een model van een half oneindige dubbelbrekende plaat met de effectieve brekingsindextensor door het model te passen op de experimentele data en daardoor verkregen de anisotropie van de effectieve brekingsindex We bestuderen de reflectiviteit van 3D fotonische kristal structuren met en zonder ingebouwde defecten. Van de waarneming van stopbanden in reflectiviteit verkrijgen we structurele eigenschappen van fotonische kristallen welke we vergelijken met de verkregen waarden uit het tomografie experiment en vinden een overeenkomst. In de reflectiviteit spectra die genomen zijn van de 3D kristallen met ingebouwde defecten nemen we zoals verwacht een trog waar binnen de berekende bandkloof. We vinden goede overeenkomst van de trog in de reflectiviteit piek met de ruimtelijke positie van het trilholte defect in het kristal. The results presented in this thesis allow to kick off fabrication and metrology for 3D silicon nanophotonics. De resultaten welke in deze dissertatie gepresenteerd worden geven aanleiding tot de start van fabricatie en metingen van $3 \mathrm{D}$ nanofotonica. 


\section{Acknowledgements}

Our group, Complex Photonic Systems (COPS) is a unique group because of the people it consist of. For building COPS that way I would like to thank all our senior members - Willem Vos, Allard Mosk, Pepijn Pinkse and Ad Lagendijk.

First I would like to thank our group leader and my supervisor Willem. Willem, thank you for being a true Teacher for me, for constantly spotting my weak points and advising on how to improve them. It was tough but always very efficient and I am deeply grateful for it. It is was a very interesting experience to have my supervisor sometimes working in a lab as a "postdoc" - measuring himself reflectivity spectra with great enthusiasm or staying on the synchrotron till late night (or better to say early morning!) and keeping a logbook (which was always curious to read later on). I learned an enormous amount of things from you that are not limited to purely science but also about communication and being a professional. You are a very special PhD supervisor and I can not imagine a better one for me. Your efforts to find a different and effective approach to every of your students and seeing personalities in each of us always amazed me. Thank you for your incredible patience, passion and enthusiasm!

Allard, I would like to thank you for your endless knowledge of every subject, ideas, suggestions and contribution to all scientific discussions during the time when your were a part of COPS. Pepijn, thank you for showing your amazing curiosity to science and teaching and especially enthusiasm to find original ways to demonstrate physics experiments to students. Ad, it is my honor that I got a chance to work in the group which you are a part of. Thank you for being you, it is impossible to describe, but the fact that I could see your way of doing, creating and communicating science is priceless. Thank you for being such an amazing and unique scientist. Cock, technician of COPS, thank you for the great help in my research, for your spirit, your kind support outside work and for the example of a man who truly knows how to live a happy life! Thank you as well for your great help in nanofabrication, etching and of course help in our tomography adventures. Our secretaries Nilda, Jacqueline, Carla, Nienke and Nicole, thank you all for dealing with all the papers, the group could not function without you. Marlon, thank you for the ICT support, with all the troubles I had with ICT I am even not sure if you will have enough work to do after I leave.

I would like to greatly acknowledge my committee members Ad Lagendijk, Jos Benschop, Frank Scheffold, Peter Cloetens, and Guus Rijnders for their time and effort to be jury of my PhD thesis.

During my PhD I was involved in multiple fruitful collaborations with other University and industrial groups. I would like to thank Jorge Perez-Vizcaino 
for the great help in reflectivity measurements. Despite the broken objective we got nice results and I had an amazing time working together with you, Jorge. Takeyoshi Tajiri and Satoshi Iwamoto for their collaboration, Takeyoshi for his inspiration to take painstakingly precise polarization data of which a first interpretation is mentioned in this thesis. Arie den Boef, Jo Finders and Eddy van der Heijden from ASML for their help in our UV lithography adventures. Together with them I would like to thank Kevin Williams, Meint Smit, Huub Ambrosius, Jeroen Bolk and Robert van de Laar for their support during my work in the cleanroom of TU/e. I would like to greatly thank colleagues from ESRF Grenoble: Peter Cloetens, Julio Cesar da Silva and Alexandra Pacureanu for their great help and support in our X-ray tomography experiments. Stefan Weber and Philip Paul from SwissLitho for guiding me around at SPIE'16 and greatly helping with their advice and experience ever after. I would like to thank entire staff of MESA + Nanolab for their incredible support while working in the Nanolab cleanroom. My special thank extends to Rene, Ton, Christiaan, Samantha, Mark, Meint and Henk. I would also like to thank all members of Applied Nanophotonics for the discussions during our monthly colloquium and yearly retrieves. Especially I would like to thank Frans Segerink for his lively interest to my work, Sonia Garcìa Blanco and Jennifer Herek for their activity as a members of Female Faculty Network Twente (FFNT).

I would also like to thank other members of "Stirring of light!" program Flavio, Fai Tong, Martin, and Henriette.

My colleagues at COPS during all this years became more than just colleagues. We had incredible time both during the work and outside work. Thank you, guys, for all the scientific and non-scientific discussions during group meetings and coffee breaks. It was a pleasure to work together with Amandev, Andy, Bas, Ben, Bill, Chris, Devashish, Duygu, Emre, Evangelos, Femi, Georgios, Hasan, Henri, Iris, Jeroen, Jochen, Julio, Jin, Klaas-Jan, Lyuba, Maryna, Mehdi, Michael, Peter, Oxan, Pim, Peilong, Pritam, Ravi, Raheleh, Reinier, Sergei, Sjoerd, Shakeeb, Simon, Sina, Thomas, Tristan, Timmo, Tom, Vanessa, Vincent, Willemijn, Wouter. It was a pleasure to supervise students Jan, Daniel and Bram, Lüders and Erik. It was also great having our neighboring group Laser Physics and Nonlinear Optics (LPNO) every day at the coffee table and I would like to thank all members of LPNO for that.

My life in Netherlands was not limited to work. I got a chance to take a part in many sport activities. I would like to thank all the Batavierenrace teams with whom I run this awesome race during this years. I would also like to deeply thank all girls from SVOD Deventer, Rhythmic gymnastics association with whom I took part in multiple national competitions and especially thank Marieke, Eva, Juliette, Lieke, Yrsa, Maud and Charlotte. Of course my deepest gratitude goes to my coach Veneta for her kind support and faith.

Completing this thesis would not be possible without support of my friends. Martijn and Maddie, my incredible 148-neighbors, thank you for all the chocolate left at my door to cheer me up, for all the fun and adventures. Sergei, thank you for your encouragement and support during moving to this country. Hasan, thank you for your amazing laugh and being always so incredibly sincere and 
honest. Emre, thank you for the nice advice that you gave me (I still remember them often!) and for your support especially during last months of your stay here. Jin, you are the person who can sense even tiniest changes in people's mood and condition. Thank you and of course your amazing wife Yue for caring. Andy, thank you for being a part of our board game, poker and racing team, as well for you willingness to listen and bring people together. Pritam, thank you for teaching me cards magic and your help especially during my father's illness, its priceless. Tristan and Evangelos, thank you guys, for being always so kind and for sharing with me tons of my favorite nuts and fruits, it cheered me a lot. Devashish, I will never forget your support in the hardest times and amazing history of our friendship ;) Ravi, you are the true source of calmness, the moment you enter the room, everything becomes calm and clear, it is amazing. Thank you! Maryna, without you I would just not survive everything that happened to me, thank you for listening, supporting and carrying some carpets ways from me. Jeroen, thank you for the gigabytes of our text messages and for sharing with me passion to airplanes, water and travel. Alyona, thank you for the amazing feeling of home and breeziness that I had every time when visiting you! Katya, thank you for being sometimes an example for me and being my friend since the time we first met in school. Lena, you are the one who knows and understands me best, thank you for being here for me, there is no need to say how much I appreciate your support. Varya, thank you for being truly a part of my family rather than a friend during all the times and that I could always rely on you.

Naturally I would like to thank my family. My father Alexander, the smartest person I have ever met, for teaching me the basics of absolutely everything I know and making me dream of science; my mother Zinaida, the person for whom even absolute lack of language is not a barrier to successfully communicate, for her love and care and for making me social, brave and ambitious; my sister Vika, the most naturally beautiful person I know, for her desperate attempts to raise a sense of style in me and for taking care that sometimes I do look like a lady more than like a physicist; my dear aunt Kate, for never giving up in teaching me kindness and patience and being with me from my childhood; my aunt Nadin for always making our house a home. My niece Iuliia, thank you for always being my little girl and for becoming my friend, also for your trust, support and being always next to me. I would also like to thank uncle Kolya, aunt Nina, Lesha, Natasha, Kirill, Lena, Ramil, Artur, Kamilla, Stas, aunt Ira, Dima, Natasha and Ilay.

At last I would like to thank my absolutely amazing Sasha for taking me by the hand through the darkest time in my life, for being more stubborn than me whenever needed, and for bringing me something to always look forward to. Having you in my life is one of the best things happened to me, thank you!

Diana 
